\title{
Identification of novel components involved in selective and unselective autophagic pathways
}

\author{
Dissertation \\ for the award of the degree \\ "Doctor rerum naturalium" \\ of the Georg-August-University Goettingen
}

submitted by

Evelyn Cornelia Welter

from Ottweiler

Goettingen 2011 
Supervisor:

Prof. Dr. Michael Thumm

Department of Biochemistry II

Center for Biochemistry and Molecular Cell Biology

Georg-August University, Goettingen

Member of the Thesis Committee (First Reviewer):

Prof. Dr. Peter Rehling

Department of Biochemistry II

Center for Biochemistry and Molecular Cell Biology

Georg-August University, Goettingen

Member of the Thesis Committee (Second Reviewer):

Prof. Dr. Gerhard Braus

Deptartment of Molecular Microbiology and Genetics

Institute for Microbiology and Genetics

Georg August University Goettingen

Member of the Thesis Committee:

Prof. Dr. Ralf Ficner

Department of Molecular Structural Biology

Goettingen Center for Molecular Biosciences

Georg August University Goettingen

Date of oral exam: 16.05 .11 
Affidavit:

I herewith declare, that this thesis has been written independently and with no other sources and aids than quoted. 



\section{Contents}

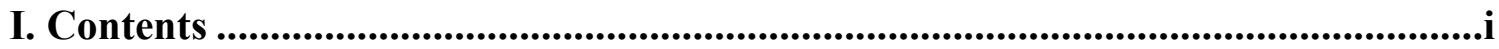

II. List of figures...............................................................................................................iv

III. List of tables ..................................................................................................................v

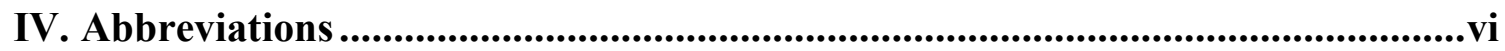

1. Summary........................................................................................................................

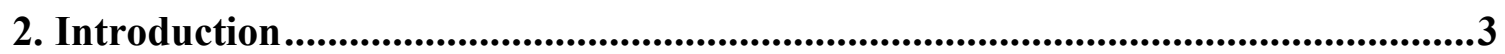

2.1 The model organism Saccharomyces cerevisiae ........................................................3

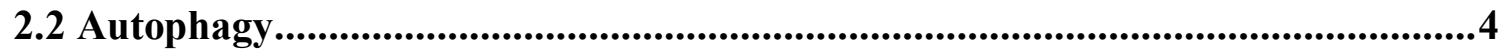

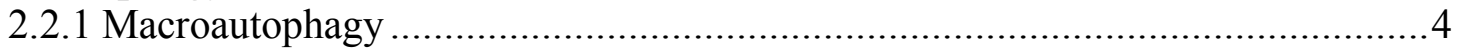

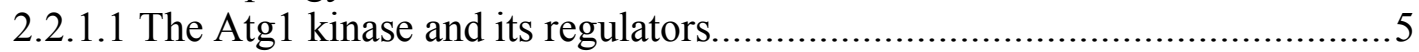

2.2.1.2 The two PtdIns 3 -kinase complexes ........................................................ 6

2.2.1.3 The pre-autophagosomal structure .................................................... 7

2.2.1.4 The two conjugation systems ........................................................ 7

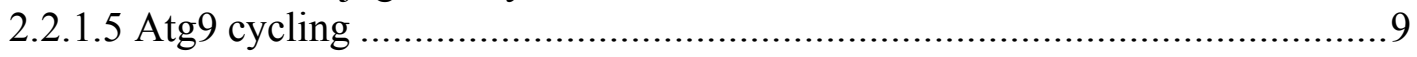

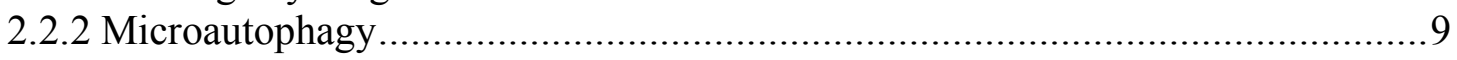

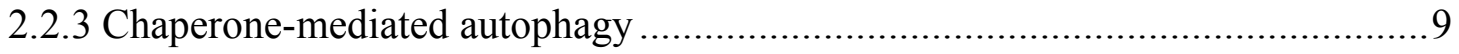

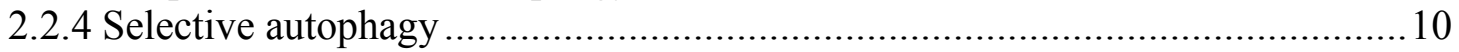

2.2.4.1 The Cytoplasm to Vacuolar Trafficking pathway ….............................. 10

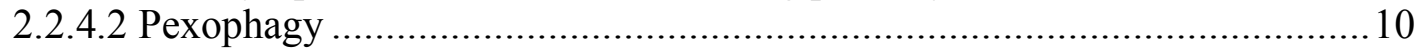

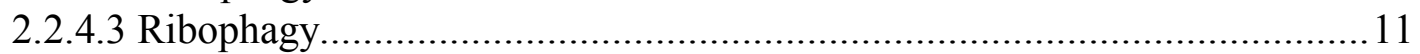

2.2.4.4 Piecemeal Microautophagy of the Nucleus............................................... 11

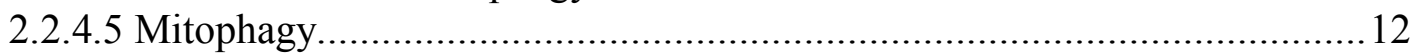

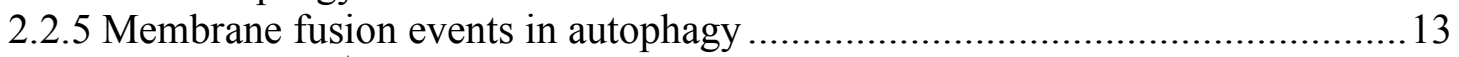

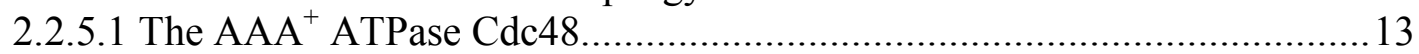

2.2.5.1.1 Cdc48 and autophagosome biogenesis ......................................... 14

2.3 The ESCRT machinery …......................................................................................15

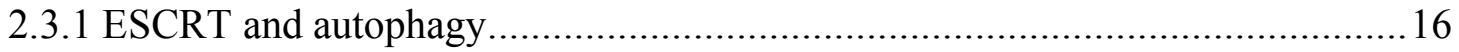

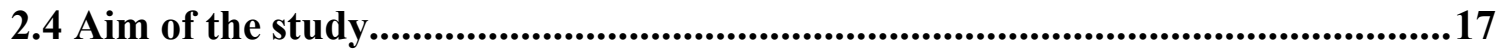

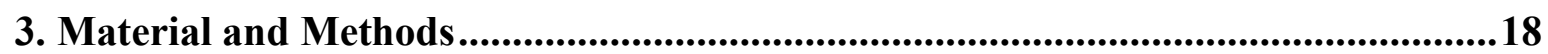

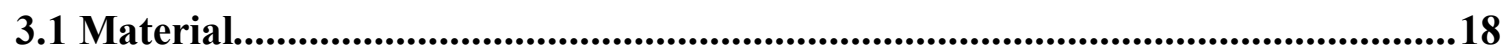

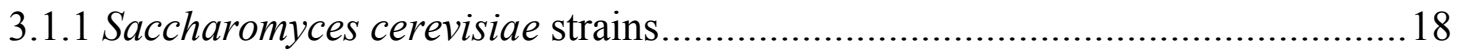

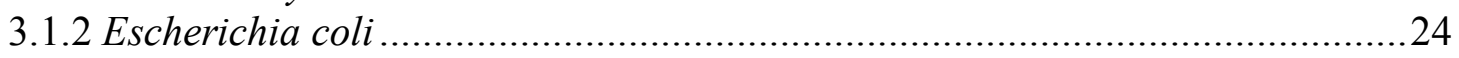

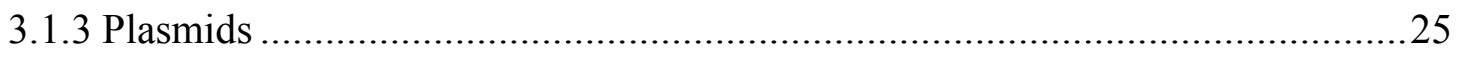

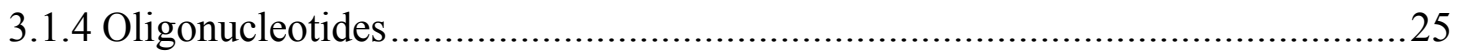

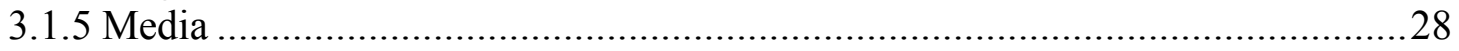

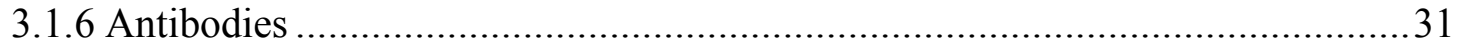

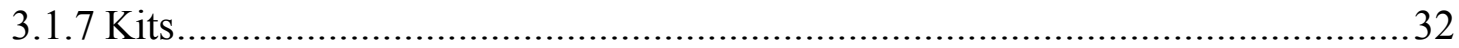

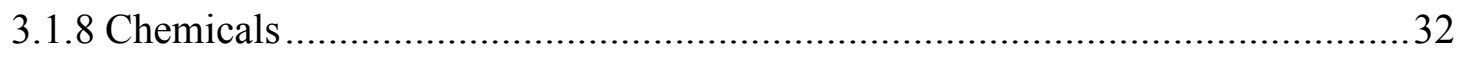

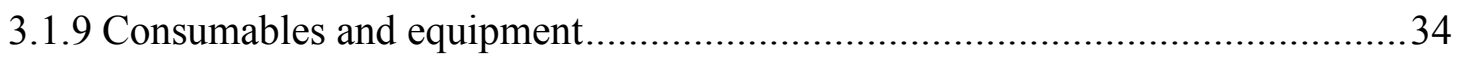




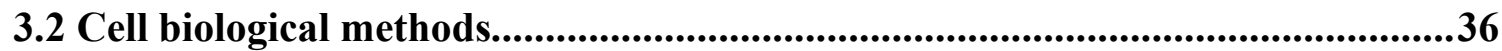

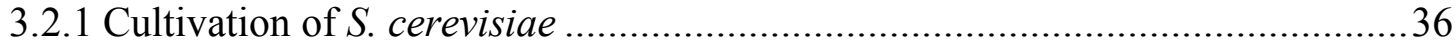

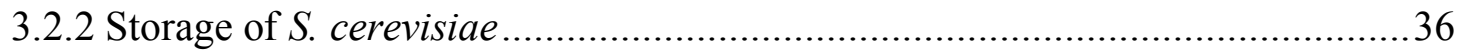

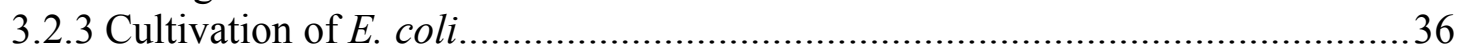

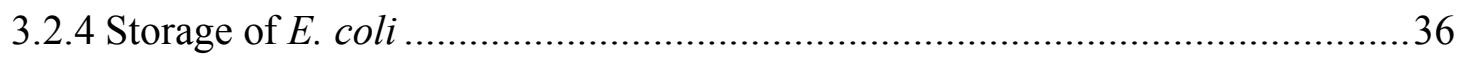

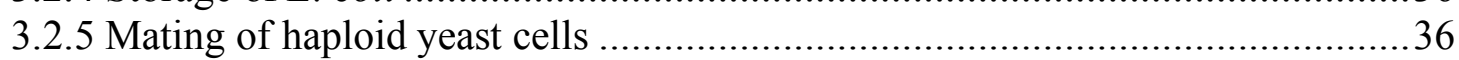

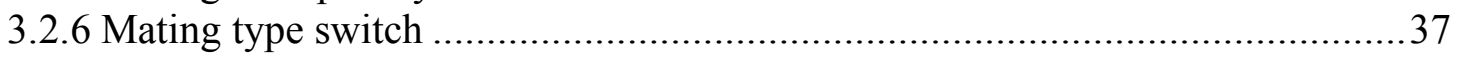

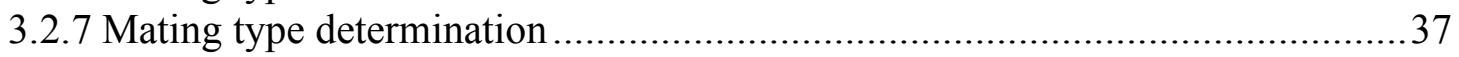

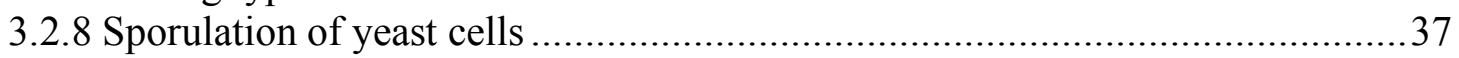

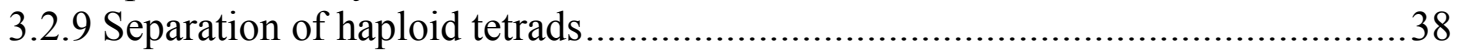

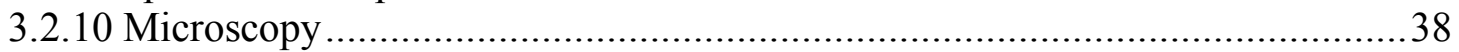

3.2.10.1 Test for the accumulation of autophagic bodies inside the vacuole ..........38

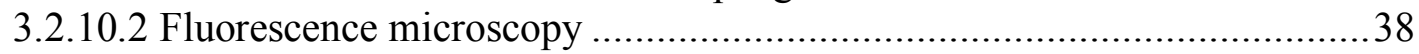

3.2.10.2.1 Staining of the nucleus using Hoechst 33324 .....................................39

3.2.10.2.2 Staining of endosome and vacuole using FM4-64 ...............................39

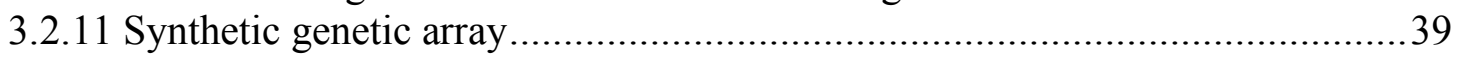

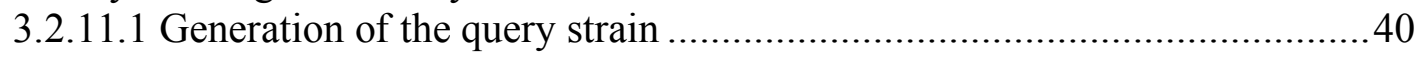

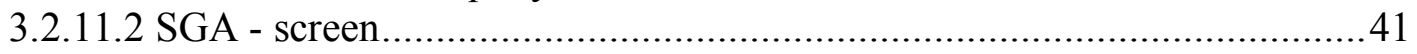

3.3 Molecular biological methods .........................................................................43

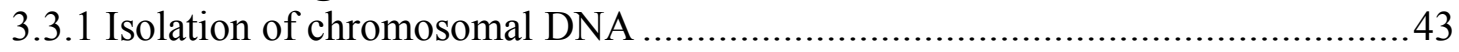

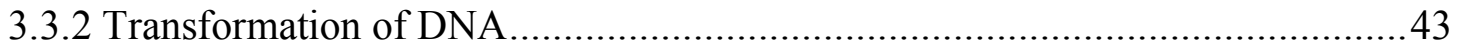

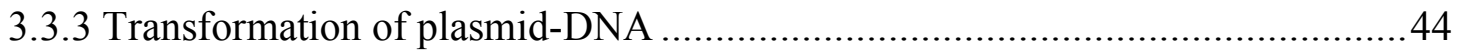

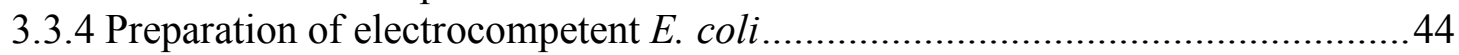

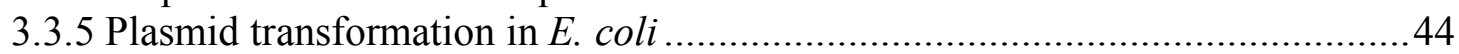

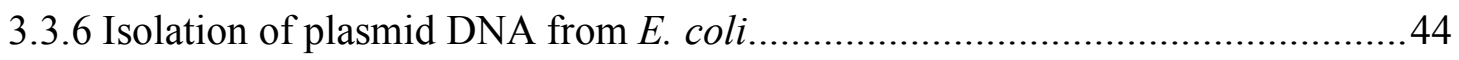

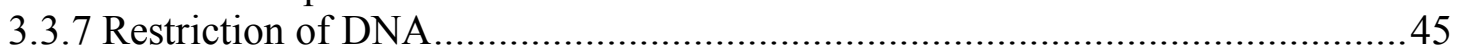

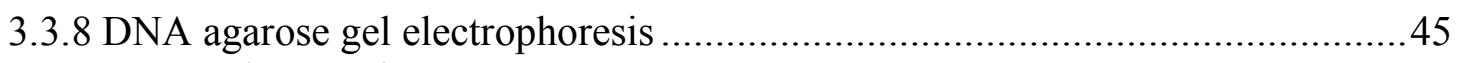

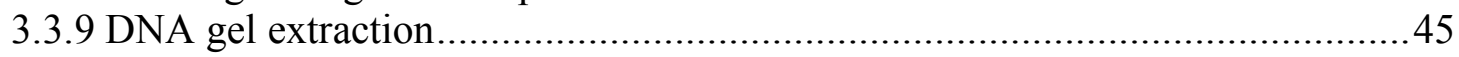

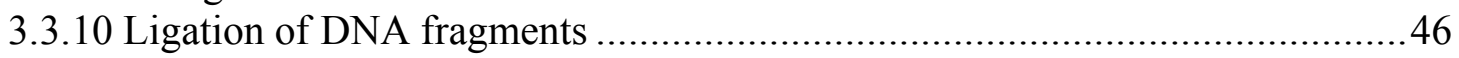

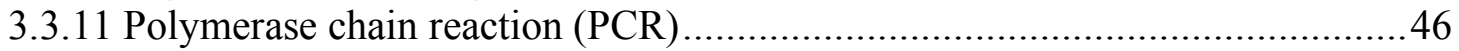

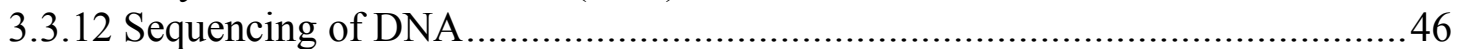

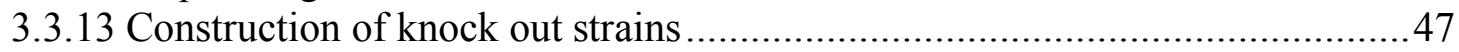

3.3.14 Chromosomal C-terminal fusion with a GFP-taq.......................................47

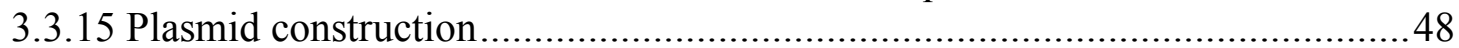

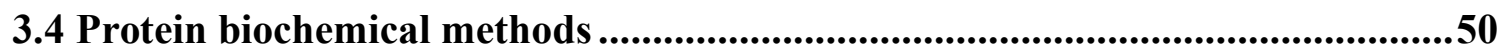

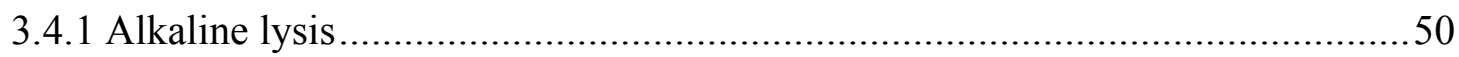

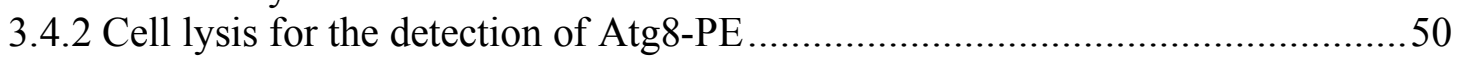

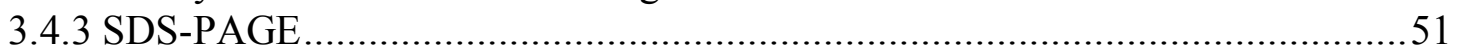

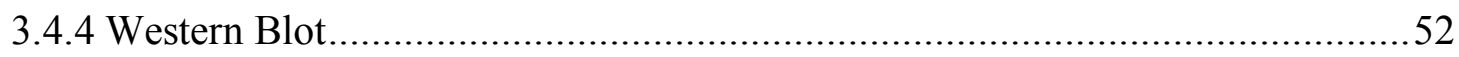

3.4.5 Measurement of autophagy dependent degradation of GFP-fused proteins .......53

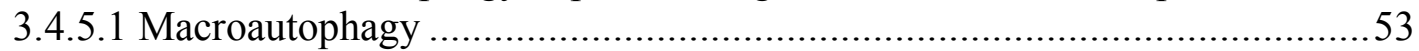

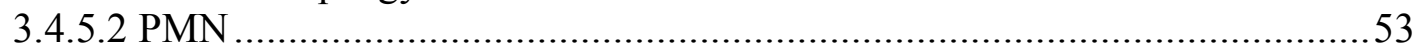

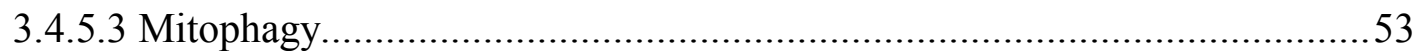

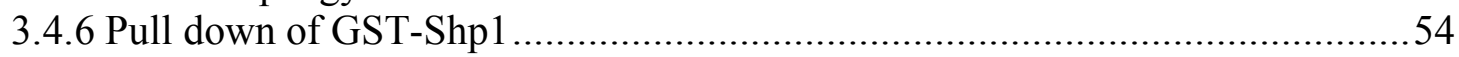

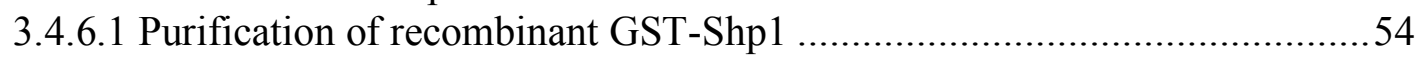

3.4.6.2 Coupling of GST-fusion protein to sepharose .........................................5

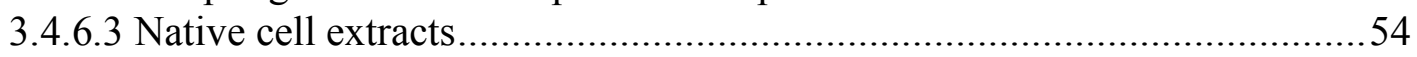

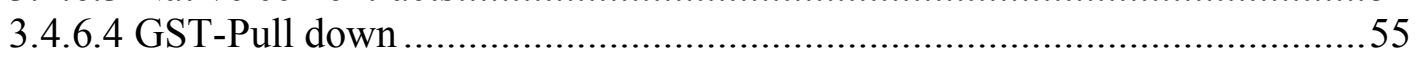




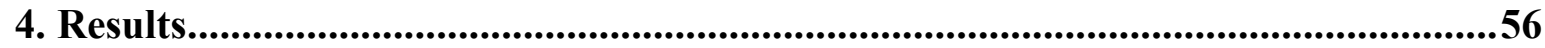

4.1 Synthetic Genetic Array...........................................................................................56

4.2 Detection of nonselective bulk autophagy using Pgk1-GFP..............................59

4.3 Cdc48 and its cofactor Shp1 are needed for functional autophagy ...................62

4.3.1 Shp1 is the exclusive cofactor of Cdc48 needed for autophagy........................62

4.3.2 Shp1 is needed for autophagy but not for the Cvt pathway and CPY maturation

4.3.4 The formation of the PAS occurs normal in $\operatorname{shp} 1 \Delta$ cells.................................67

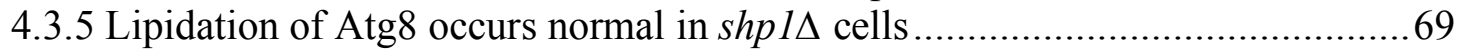

4.3.6 Pull down experiments of recombinant GST-Shp1 .....................................69

4.4 Mitophagy in S. cerevisiae ..........................................................................71

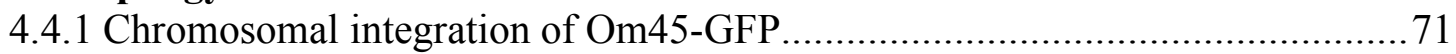

4.4.2 The pre-autophagosomal structure localises to mitochondria..........................72

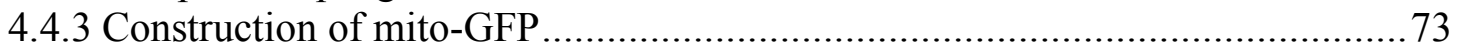

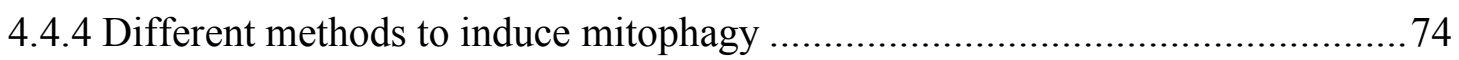

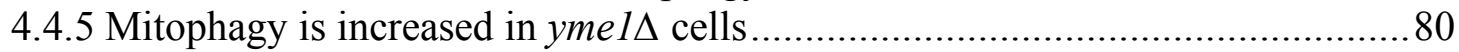

4.4.6 Part of the ESCRT machinery is required for mitophagy ................................8 81

4.4.7 Doa4 is involved in mitophagy but not in unselective autophagy......................83

4.5 Piecemeal microautophagy of the nucleus.......................................................85

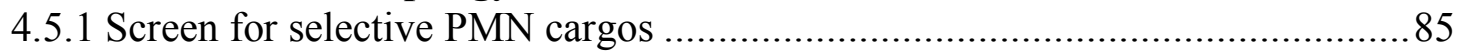

4.5.2 Microscopic analysis of nucleolar GFP fusion proteins .................................86

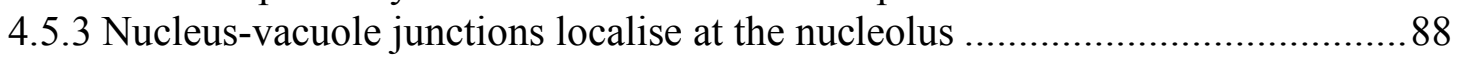

4.5.4 The PAS localises at the nucleolar part of the nucleus...................................90

5. Discussion ........................................................................................................................91

5.1 Synthetic Genetic Array (SGA) ...............................................................................91

5.2 Establishment of a novel assay to easily measure unselective autophagy using Pgk1-GFP ........................................................................................................................95

5.3 A complex of Ccd48, Shp1 and Atg8 is needed for autophagosome biogenesis in

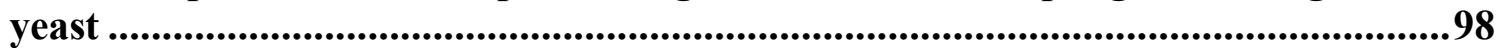

5.3.1 Shp1 is the exclusive cofactor of Cdc48 needed for autophagy.......................98

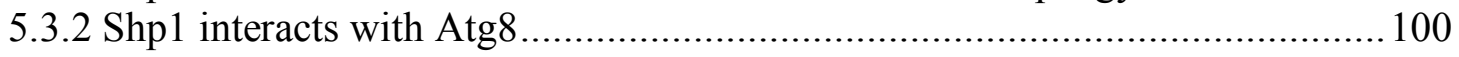

5.4 Insights into mitophagy in $S$. cerevisiae ......................................................103

5.4.1 Differentiation between mitophagy of superfluous or damaged mitochondria. 105

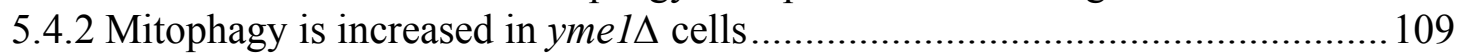

5.4.3 Part of the ESCRT machinery is required for mitophagy ............................. 110

5.4.4 Ubiquitination affects mitophagy in $S$. cerevisiae ....................................... 111

5.5 The nucleolus might represent a specific cargo for PMN ...............................114

6. Literature...................................................................................................................117 


\section{List of figures}

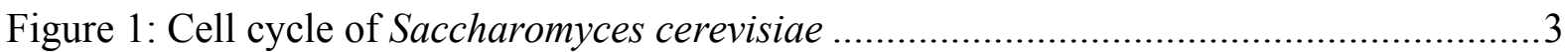

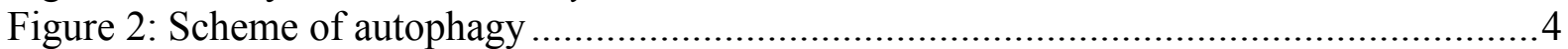

Figure 3: The regulatory TOR kinase complex for autophagy induction ............................6

Figure 4: The two Phosphatidylinositol 3-kinase complexes in S. cerevisiae .........................7

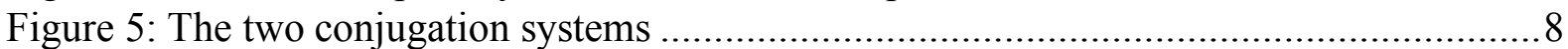

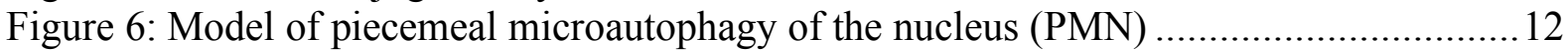

Figure 7: Model of Cdc48-Shp1 complex..................................................................... 14

Figure 8: The ESCRT machinery of MVB sorting pathway ......................................... 16

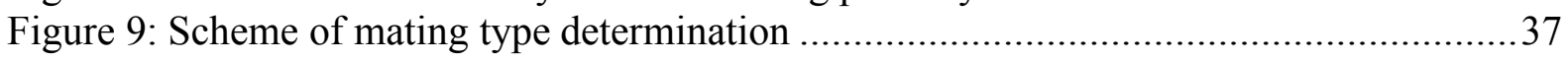

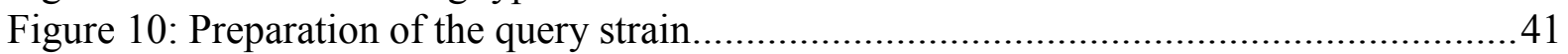

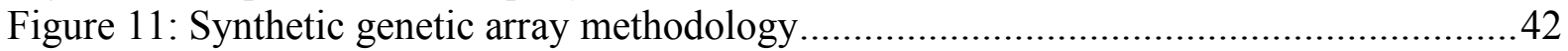

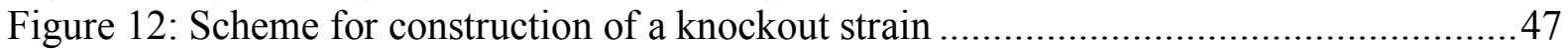

Figure 13: Scheme for the construction of chromosomal Om45-GFP .............................48

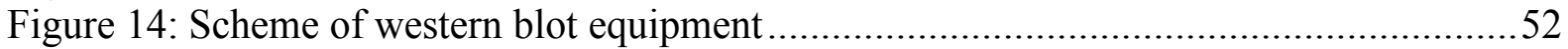

Figure 15: Mating and sporulation for query strain generation was controlled by microscopy

Figure 17: Monitoring unselective autophagy using Pgk1-GFP .......................................60

Figure 18: Fluorescence microscopy of cells expressing Pgk1-GFP .................................61

Figure 19: Ubx1 but no other UBX domain protein is required for autophagy ....................62

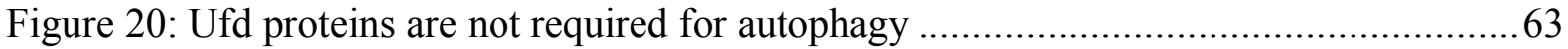

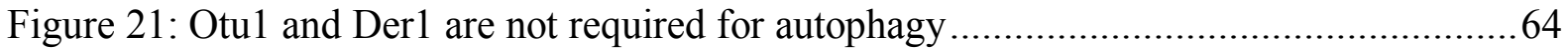

Figure 22: Macroautophagy is not affected in doa $4 \Delta$ cells.................................................64

Figure 23: Shp1 is required for autophagy but not for the Cvt-pathway ............................65

Figure 24: The maturation of Ape1 and CPY occurs normal in shp $1 \Delta$ cells .......................66

Figure 25: No vacuolar accumulation of autophagic bodies in $\operatorname{shp} 1 \Delta$ cells ........................67

Figure 26: The formation of the PAS occurs normal in $\operatorname{shp} 1 \Delta$ cells .................................68

Figure 27: The lipidation of Atg8 occurs normally in $\operatorname{shp} 1 \Delta$ cells..................................69

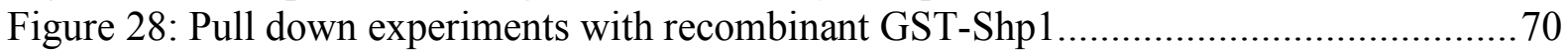

Figure 29: Monitoring mitophagy by western blot using Om45-GFP ................................72

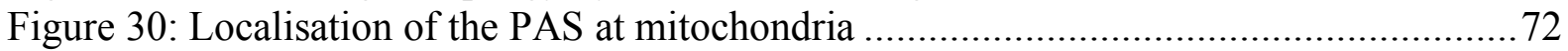

Figure 31: Fluorescence microscopy and western blot analysis of cells expressing mito-GFP

Figure 32: Incubation for 3 days in lactate medium induces mitophagy ............................ 75

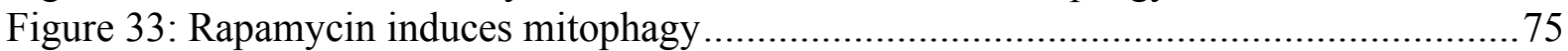

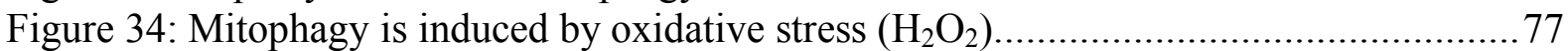

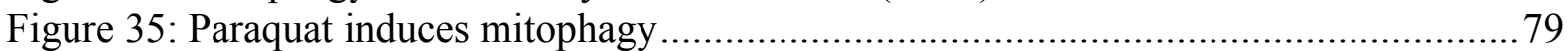

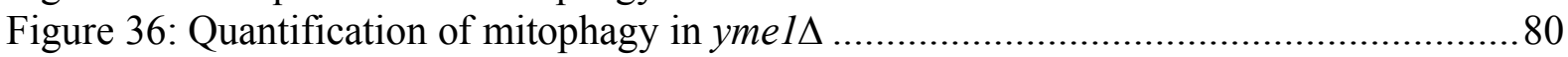

Figure 37: Part of the ESCRT machinery is required for mitophagy ................................. 82

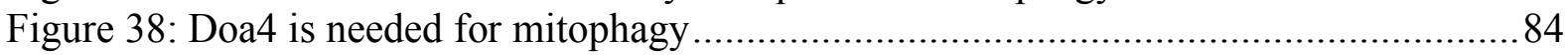

Figure 39: Western Blot analysis for the identification of specific PMN cargos...................86

Figure 40: Microscopic analysis of strains expressing nucleolar GFP fusion proteins ..........87

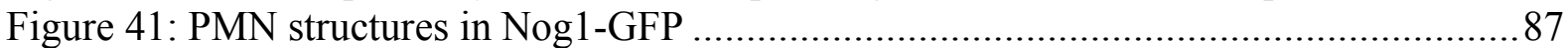

Figure 42: Nucleus-vacuole junctions localise adjacent the nucleolus................................89

Figure 43: The pre-autophagosomal structure localises partially at the nucleolus.................90 


\section{List of tables}

Table 1: Strains of BY4741 background used in this study ........................................... 18

Table 2: Strains of BY4742 background used in this study ............................................ 19

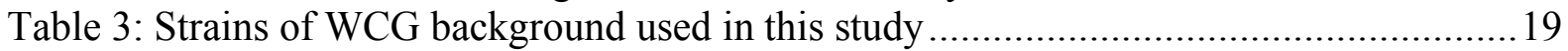

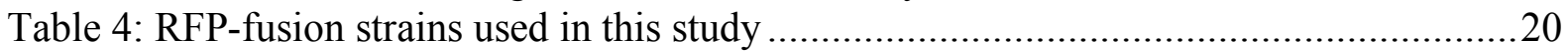

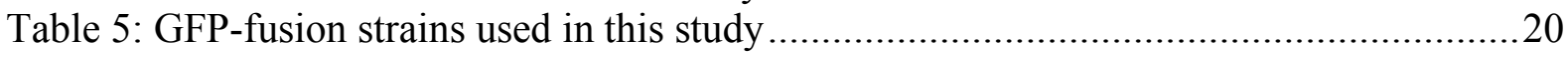

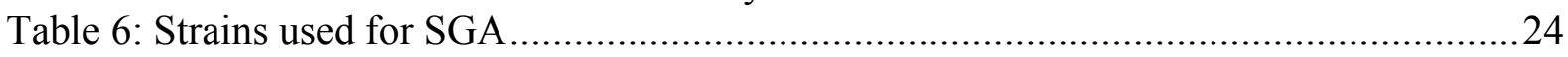

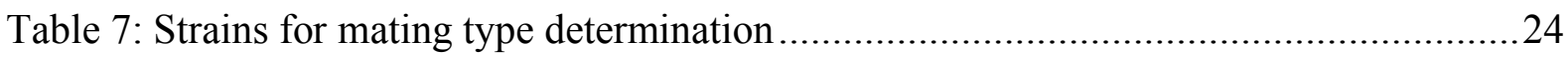

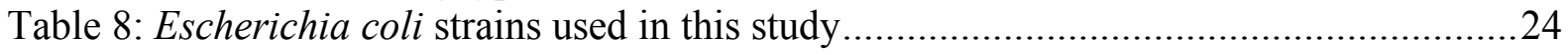

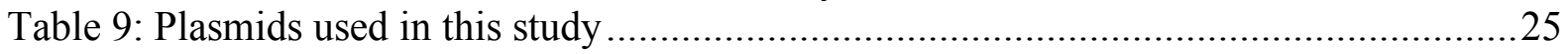

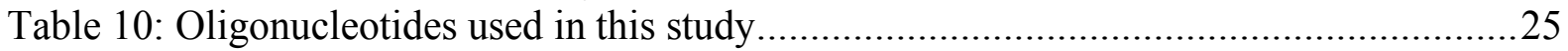

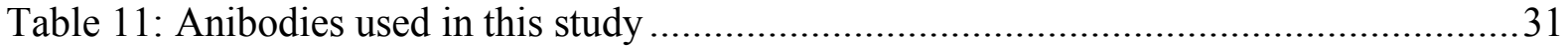

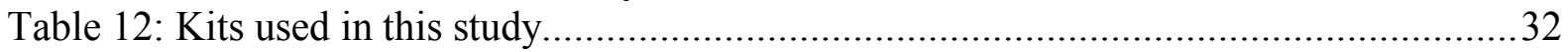

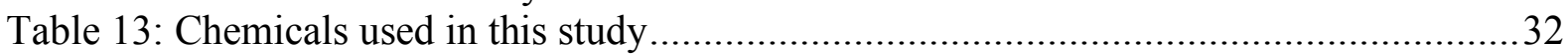

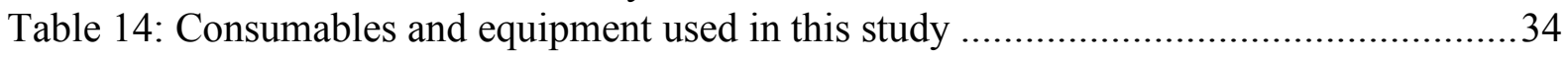

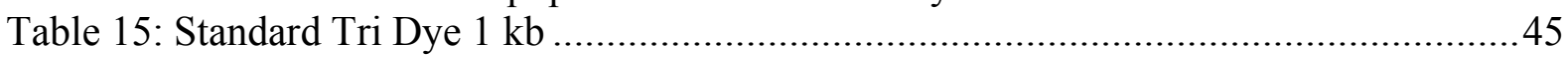

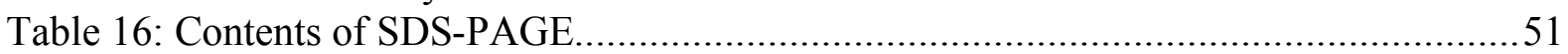

Table 17: Precision Plus Protein All Blue Standard ......................................................... 51

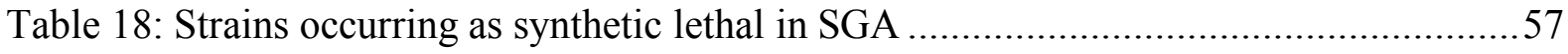

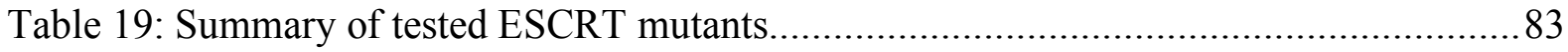




\begin{tabular}{|c|c|}
\hline \multicolumn{2}{|l|}{ Abbreviation } \\
\hline$\%$ & Per cent \\
\hline$\Sigma$ & Sum \\
\hline$(\mathrm{v} / \mathrm{v})$ & (Volume/volume) \\
\hline$(w / 0)$ & Without \\
\hline$(w / v)$ & (Weight/volume) \\
\hline${ }^{\circ} \mathrm{C}$ & Degree in celcius \\
\hline $\mathrm{AAA}^{+}$ATPase & ATPases associated with various cellular activities \\
\hline $\mathrm{AB}$ & Autophagic body \\
\hline amp & Ampicillin \\
\hline Ams 1 & $\alpha$-mannosidase \\
\hline AP & Autophagosome \\
\hline Ape1 & Aminopeptidase1 \\
\hline APS & Ammonium persulfate \\
\hline $\arg$ & Arginine \\
\hline atg & Autophagy related gene \\
\hline bp & Base pairs \\
\hline BSA & Bovine serum albumine \\
\hline can & Canavanine \\
\hline clonNAT & Nourseotricine \\
\hline $\mathrm{CM}$ & Complete minimal medium \\
\hline $\mathrm{CM}$ & Chloramphenicol \\
\hline CPY & Carboxypeptidase Y (Pcr1) \\
\hline Cvt & Cytosolic to vacuolar \\
\hline DMA & Deletion mutant array \\
\hline DMSO & Dimethylsulfoxide \\
\hline DNA & Deoxyribonucleic acid \\
\hline dNTP & Deoxynucleosidetriphosphate (dATP, dGTP, dCTP, dTTP) \\
\hline DO & Dropout mix \\
\hline E. coli & Escherichia coli \\
\hline e.g. & For example \\
\hline ECL & Enhanced chemiluminescence \\
\hline EDTA & Ethylenediamintetraacedic acid \\
\hline ERAD & Endoplasmic reticulum associated protein degradation \\
\hline ESCRT & Endosomal sorting complex required for transport \\
\hline $\mathrm{EtOH}$ & Ethanole \\
\hline fig. & Figure \\
\hline $\mathrm{g}$ & Gramm \\
\hline $\mathrm{g}$ & Gravity \\
\hline G418 & Kanamycin \\
\hline GFP & Green fluorescent protein \\
\hline GST & Glutathione S-transferase \\
\hline $\mathrm{h}$ & Hour \\
\hline H. polymorpha & Hansenula polymorpha \\
\hline $\mathrm{H}_{2} \mathrm{O}$ & Dihydrogen monoxide (water) \\
\hline $\mathrm{H}_{2} \mathrm{O}_{2}$ & Hydorgen peroxide \\
\hline his & Histidine \\
\hline
\end{tabular}




\begin{tabular}{|c|c|}
\hline Abbreviation & \\
\hline -his & Without histidine \\
\hline -his/arg/lys & Without histidine/arginine and lysine \\
\hline HOPS & Homotypic fusion and vacuole protein sorting \\
\hline ILV & Intralumenal vesicles \\
\hline IPTG & Isopropyl- $\alpha$-D-Thiogalactopyranoside \\
\hline KAN & Kanamycin \\
\hline $\mathrm{kb}$ & Kilo bases \\
\hline $\mathrm{kDa}$ & Kilo dalton \\
\hline $\mathrm{kg}$ & Kilo gramm \\
\hline 1 & Liter \\
\hline leu & Leucine \\
\hline -leu/arg/lys & Without leucine/arginine and lysine \\
\hline lyp & Thialysine \\
\hline lys & Lysine \\
\hline M & Molar \\
\hline $\mathrm{mA}$ & Milli ampere \\
\hline- met & Without methionine \\
\hline $\min$ & Minutes \\
\hline mito & Mitochondrial \\
\hline $\mathrm{ml}$ & Milli liter \\
\hline $\mathrm{mM}$ & Milli molar \\
\hline MV & Minimal medium \\
\hline MVB & Multi vesicular body \\
\hline $\mathrm{n}$ & Number of independent experiments \\
\hline NADPH & Nicotinamidadenindinukleotidphosphat \\
\hline NAT & Nourseotricine (clonNAT) \\
\hline NEB & New england biolabs \\
\hline $\mathrm{nm}$ & Nano meter \\
\hline NSF & N-ethylmaleimide-sensitive factor \\
\hline NV junction & Nuclear vacuolar junction \\
\hline $\mathrm{O}_{2}^{-}$ & Superoxide anion \\
\hline OD & Optical indensity \\
\hline ORF & Open reading frame \\
\hline OSBP & Oxysterol-binding protein \\
\hline $\mathrm{p}$ & Plasmid \\
\hline paraquat & N, N'-dimethyl-4,4'-bipyridinium dichloride \\
\hline P. pastoris & Pichia pastoris \\
\hline PAGE & Poly acryl amide gel electrophoresis \\
\hline PAS & Pre-autophagosomal structure \\
\hline PBS & Phosphate buffered saline \\
\hline PCR & Polymerase chain reaction \\
\hline PE & Phosphatidylethanolamine \\
\hline $\mathrm{pH}$ & Negative logarithm of $\mathrm{H}+$ concentration \\
\hline $\mathrm{PMN}$ & Piecemeal microautophagy of the nucleus \\
\hline PMSF & Phenylmethylsulfonylfluoride \\
\hline PVDF & Polyvinylidene Fluoride \\
\hline RFP & Red fluorescent protein \\
\hline RNA & Ribonucleic acid \\
\hline ROS & Reactive oxygen species \\
\hline $\mathrm{rpm}$ & Rounds per minute \\
\hline
\end{tabular}




\begin{tabular}{|c|c|}
\hline \multicolumn{2}{|l|}{ Abbreviation } \\
\hline RT & Room temperature \\
\hline s & Seconds \\
\hline S.cerevisiae & Saccharomyces cerevisiae \\
\hline SD & Synthetic minimal medium \\
\hline SD-N & Synthetic minimal medium lacking nitrogen $(\mathrm{SD}(-\mathrm{N}))$ \\
\hline SDS & Sodium dodecyl sulfate \\
\hline sec & Secretory pathway \\
\hline SEM & Standard error of the mean \\
\hline SGA & Synthetic genetic analysis \\
\hline SNAP & Synaptomal-associated protein \\
\hline SNARE & Soluble NSF attachment receptor \\
\hline SOC & Super optimal broth containing glucose \\
\hline ß-ME & Beta mercaptoethanole \\
\hline tab. & Table \\
\hline TAE & Tris-acetate-EDTA-buffer \\
\hline Taq & Thermus aquaticus \\
\hline TBST & Tris buffered saline with Tween \\
\hline TCA & Trichloroacatic acid \\
\hline TEMED & $\mathrm{N}, \mathrm{N}, \mathrm{N}^{\prime}, \mathrm{N}^{\prime}$-Tetramethylethylenediamine \\
\hline TOR & Target of rapamycin \\
\hline $\mathrm{U}$ & Units \\
\hline ura & Uracile \\
\hline -ura & Without uracile \\
\hline UV & Ultraviolet \\
\hline $\mathrm{V}$ & Volt \\
\hline YPD & Yeast Peptone Dextrose \\
\hline YPL & Yeast Peptone Lactate \\
\hline $\mathrm{V}_{\max }$ & Maximum speed \\
\hline Vps & Vacuolar protein sorting \\
\hline WT & Wild type \\
\hline YPD & Yeast extract peptone dextrose \\
\hline YPL & Yeast extract peptone lactate \\
\hline$\mu \mathrm{g}$ & Micro gramm \\
\hline$\mu 1$ & Micro liter \\
\hline$\mu \mathrm{M}$ & Micro molar \\
\hline
\end{tabular}




\section{Summary}

Autophagy is a ubiquitous protein degradation pathway in eukaryotic cells. As response upon nutrient starvation, cytosolic material and also whole organelles are engulfed by a double membrane-layered vesicle, the autophagosome. The formation of the autophagosome starts at the pre-autophagosomal structure (PAS). The completed autophagosome is then targeted to the vacuole. After fusion with the vacuole, a single membrane-layered vesicle, the autophagic body, is released into the vacuole. After lysis of the membrane, the contents of the autophagic body are degraded and the contained macromolecules are recycled. The aim of this study was the identification of cooperating or redundant genes to different autophagic genes, to uncover molecular details of autophagosome formation and to allow mechanistic insights into different selective variants of autophagy.

For the identification of autophagy related cooperating or redundant genes, the Synthetic Genetic Array analysis (SGA) was used. This is a powerful high-throughput technique to identify synthetic lethal double deletions, probably reflecting the same essential function of the respective gene products. For this purpose, the query strains $\operatorname{atg} 8 \Delta, \operatorname{atg} 15 \Delta, \operatorname{atg} 18 \Delta$ and $\operatorname{atg} 21 \Delta$ were generated in this study and crossed to the entire deletion library available for $S$. cerevisiae. Unfortunately, the procedure resulted in only false positive matches.

In addition to the common marker protein GFP-Atg8, this study introduces the marker protein Pgk1-GFP to detect unselective autophagy by western blot and microscopy. Compared to the autophagic substrate GFP-Atg8, the use of this cytosolic protein allows the unbiased detection of unselective bulk autophagy.

The molecular details of the biogenesis of autophagosomes are poorly understood. Our group identified the $\mathrm{AAA}^{+}$ATPase Cdc48 and its cofactor Ubx1 (Shp1) as novel components needed for autophagosome biogenesis. In accordance with former experiments in the laboratory, this study presents Shp1 as the exclusive cofactor of Cdc48 needed for autophagy. SHP1 deleted cells show no accumulation of autophagosomes in the vacuole, while the formation of the PAS or the lipidation of Atg8, which both are important steps during autophagosome formation, are not impaired in shpls cells. Furthermore, a suggested interaction of Shp1 and Atg8 was proven by recombinant pull down experiments using GSTShp1 and some truncated versions. In mammals, the homologues of Cdc48 and Shp1, p97 and p47, are involved in the ubiquitin-dependent mitotic Golgi reassembly. In contrast, the complex of $\operatorname{Cdc} 48$ and Shp1 in yeast, requires the ubiquitin-like Atg8 for its function in 
autophagy. This gives for the first time a rationale for the use of Atg8 during unselective autophagy and explains why the delipidation of Atg8-PE by Atg4 is necessary for efficient autophagy.

Mitochondria are the sites of ATP production in aerobic cells. As a consequence, they are at the same time a major source for reactive oxygen species (ROS), which damage mitochondria and other organelles. Thus, a quality control for mitochondria is essential for cellular survival. Mitophagy, the selective autophagic degradation of mitochondria, might fulfil such a role, but is still poorly understood in yeast. In this study, the construction of a marker protein and its use in degradation assay experiments by western blot allow the quantitative measurement of mitophagy. In contrast to a commonly used chromosomal Om45-GFP fusion, the novel marker protein can be expressed vom a plasmid, avoiding time consuming chromosomal integration. Different conditions to induce mitophagy were established. These different conditions allow the differentiation between mitophagy of superfluous or damaged mitochondria. Hereby, proteins of the ESCRT machinery, not required for unselective bulk autophagy, were found to be needed for mitophagy. In humans, Parkin ubiquitinates mitochondrial substrates to induce mitophagy. In this study, the ubiquitin isopeptidase Doa4 is shown to be required for mitophagy, indicating for the first time a mitophagy-related role of ubiquitin in yeast.

Another selective variant of autophagy is PMN, the piecemeal microautophagy of the nucleus. A specific cargo-recognition in PMN is indispensable to preserve the integrity of the nucleus. Therefore, the still unknown nuclear cargo was a focus of interest. The results of this study point to a specific cargo-recognition of nucleolar proteins in PMN. Strains expressing different nucleolar GFP fusion proteins were investigated, some showed PMN-like structures in microscopy. Furthermore, nucleus-vacuole junctions, the contact sites between the nucleus and the vacuolar membrane, are shown to localise near the nucleolus. 


\section{Introduction}

\subsection{The model organism Saccharomyces cerevisiae}

Saccharomyces cerevisiae is a budding yeast and belongs to the family of the Saccharomycetae. It is one of the best-studied eukaryotic model organisms in molecular and cell biology, comparable to the procaryotic counterpart Escherichia coli.

The cells have a size of about $5-10 \mu \mathrm{m}$ and can either be haploid or diploid. In both forms the cells can undergo a mitotic division by forming a bud. Furthermore, upon nutrient limitation a diploid cell, which results out of the mating between two haploid cells (a and $\alpha$ ), also undergoes meiotic division by sporulation. This leads to the formation of an ascus containing four haploid spores, two of each mating type (Mat a and $\alpha$ ) (Figure 1).

The genome of $S$. cerevisiae is completely sequenced and has 16 chromosomes consisting of about $13000000 \mathrm{bp}$ coding for about 5800 genes (Mewes, 1997).

As most of the proteins are highly conserved, information can often be transferred to higher eukaryotic cells, therefore $S$. cerevisiae is one of the major model organisms in cell- and developmental biology.

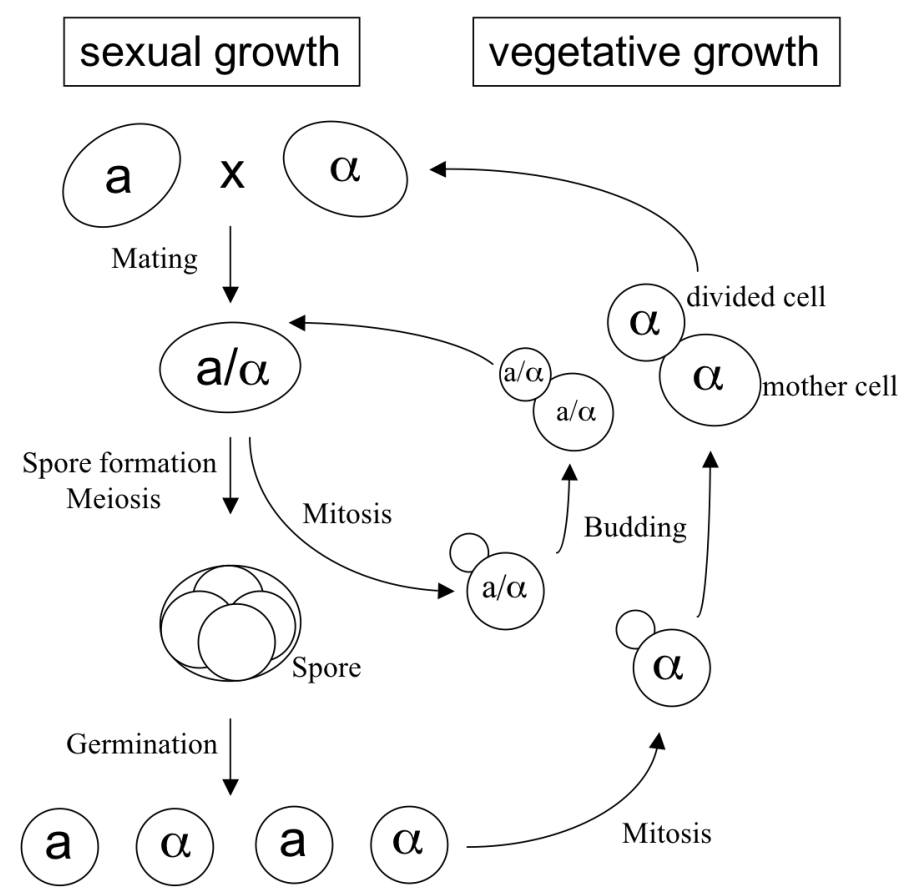

Figure 1: Cell cycle of Saccharomyces cerevisiae ((Herskowitz, 1988); modified) 


\subsection{Autophagy}

All eukaryotic cells share a major intracellular degradative pathway. This pathway, called autophagy, delivers cytoplasm, including whole organelles, to the vacuole for their degradation. Therefore, autophagy plays an important role in the recycling of cellular building blocks (Figure 2). In contrast to the selective ubiquitin dependent degradation of short-lived proteins via the proteasome, autophagy is a starvation induced, unselective or selective, degradation process of long-lived proteins and can be divided into different subtypes: macroautophagy, microautophagy and chaperone mediated autophagy.

Proteins involved in autophagy are termed Autophagy related proteins (Atg) (Klionsky, 2003). In the last decades 34 Atg proteins were identified (Suzuki; Farre, 2009; Kanki, 2009b; Okamoto, 2009).

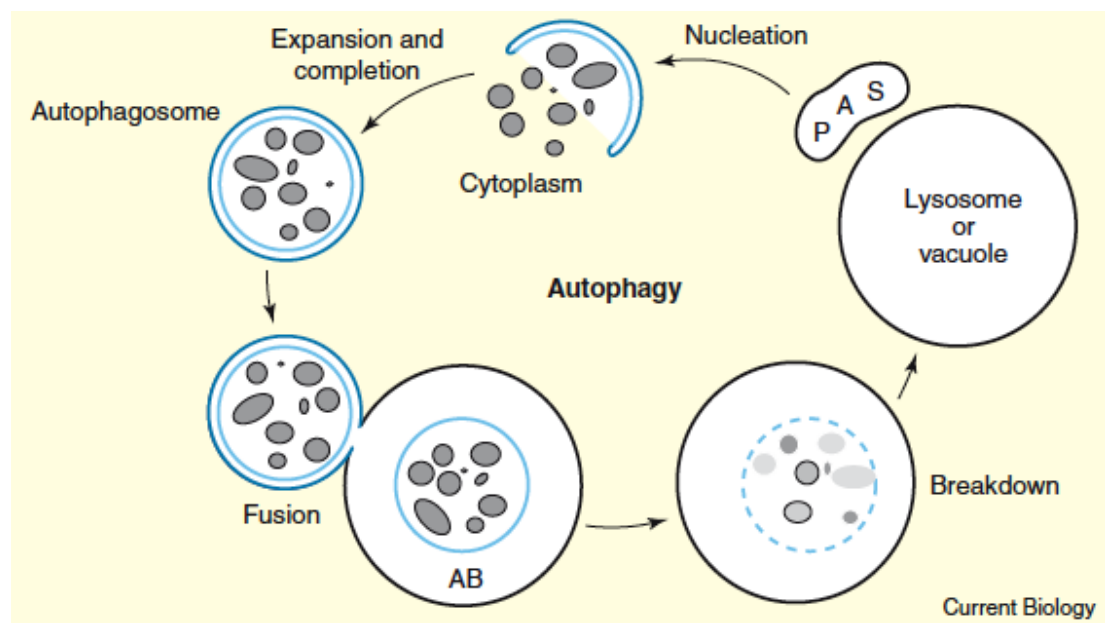

Figure 2: Scheme of autophagy

A double-membrane-layered vesicle, the autophagosome, sequesters cytoplasm, fuses with the lysosome or vacuole and a single-membrane autophagic body (AB) is released into the lysosome/vacuole where it is lysed and the cargo is degraded for the recycling of the resulting macromolecules (Klionsky, 2005).

\subsubsection{Macroautophagy}

Macroautophagy is a starvation-induced process resulting in the formation of double membrane-layered vesicles, of $300-900 \mathrm{~nm}$ in diameter. These vesicles, called autophagosomes, are formed out of a phagophore at the pre-autophagosomal structure (PAS) and engulf unselectively cytosolic material and also whole organelles by expansion of the phagophore (Takeshige, 1992; Ishihara, 2001).

The outer membrane of the autophagosome fuses with the vacuole and a still single membrane-layered vesicle, called autophagic body, is released into the vacuole (Baba, 1994). 
This fusion event of the autophagosome and the vacuole requires the homotypic vacuolar fusion machinery (Ishihara, 2001). The membrane of the autophagic body is lysed inside the vacuole and the cytoplasm-derived materials are degraded by vacuolar hydrolases. Up to now, the only identified enzyme, involved in the intravacuolar lysis, is the putative lipase Atg15, which is transported to the vacuole via the multi vesicular body (MVB) pathway (Epple, 2001; Epple, 2003). Macroautophagy, further named unselective autophagy, is up regulated under longer periods (several hours) of nutrient starvation as an adaptive response to recycle amino acids for survival. This bulk degradation of cytosolic material needs a set of Atg proteins, which belong to parts of different subcomplexes.

\subsubsection{The Atg1 kinase and its regulators}

The target of rapamycin (TOR) kinase is a negative regulator of autophagy and responds specifically to rapamycin or nitrogen starvation via an unknown upstream sensor (Figure 3). It controls the phosphorylation state of Atg13, one of the core Atg proteins. Under nutrient rich conditions the TOR kinase is active. Atg13 is highly phosphorylated in a TOR-dependent manner and therefore has low affinity to Atg1. Under nitrogen starvation the TOR kinase is inactivated and Atg13 is rapidly dephosphorylated. Therefore Atg13 associates with Atg1 as a result of a higher affinity. This interaction of $\operatorname{Atg} 13$ and Atg1 somehow regulates and enhances the serine/ threonine kinase activity of Atg1, which is essential for autophagy.

Further proteins interact with the Atg1-Atg13 complex, such as Vac8, Atg11 or Atg17. Atg11, needed for both Cvt pathway and pexophagy but not for unselective autophagy, appears to function as an adaptor or scaffold protein and allows the selective cargo recognition (Kim, 2001). Vac8 and Atg11 are also phosphorylated but independently of the Atg1-Atg13 complex. The role of their phosphorylation is unknown (Scott, 2000). Atg17 is essential for autophagy and not for the Cvt pathway, by somehow regulating the size of the forming vesicles (Kabeya, 2005). The kinase activity of Atg1 is not essential for the recruitment of all Atg proteins to the pre-autophagosomal structure (PAS). ATG1 deleted cells show an accumulation of e.g. Atg8, while Atg2 is absent from the PAS (Yorimitsu and Klionsky, 2005; Nakatogawa, 2009). The Atg1 kinase regulators Atg17, Atg29 and Atg31 function in the initial step of autophagosome formation, they are autophagy specific genes and from a ternary complex. This complex associates with the Atg1-Atg13 complex in response to nutrient starvation and is important for the activation of Atg1. This association has an essential function in the organisation of the PAS by recruitment of other core Atg 
proteins to the PAS, suggesting a function in autophagosome formation (Kawamata, 2008; Nakatogawa, 2009).

An alternative activation of Atg1 in an Atg13-independent manner under respiratory growth conditions is suggested, but still unclear (Okamoto, 2009).

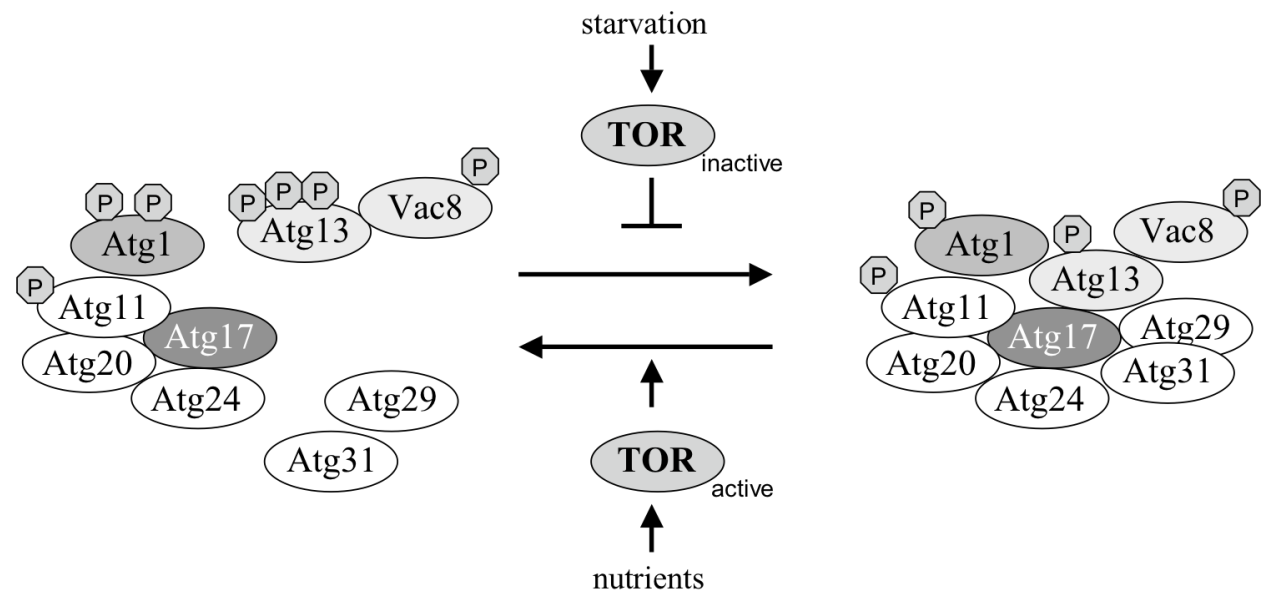

Figure 3: The regulatory TOR kinase complex for autophagy induction

((Yorimitsu and Klionsky, 2005; Nakatogawa, 2009); modified)

\subsubsection{The two PtdIns 3 -kinase complexes}

Phosphatidylinositol 3-phosphate is essential for autophagy. Vps34 is the only identified PtdIns 3-kinase in yeast and part of at least two different PtdIns 3-kinase complexes. Complex I consists, additionally to Vps34, of Vps15, Atg6 (Vps30) and Atg14, while complex II in contrast, includes Vps38 instead of Atg14 (Figure 4 (A) and (B)).

Complex I plays an important role in autophagy and the Cytoplasm to vacuolar trafficking (Cvt) pathway, therefore the deletion of ATG14 causes a block in autophagy and the Cvt pathway. The deletion of VPS38, the essential factor of complex II, leads to a block in the multivesicular body (MVB) pathway and the sorting of carboxypeptidase Y (CPY) (Kihara, 2001; Yorimitsu and Klionsky, 2005). Early data have indicated, that the PtdIns 3-kinase complex I is essential for the recruitment of Atg8-PE and the Atg12-Atg5-Atg16 conjugate (see 2.1.4) to the pre-autophagosomal structure (Suzuki, 2001; Yorimitsu and Klionsky, 2005; Klionsky, 2007; Nakatogawa, 2007; Nakatogawa, 2009; Inoue and Klionsky, 2010). 
A

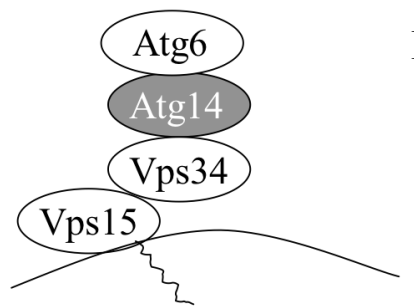

Complex I

Autophagy

Cvt pathway
B

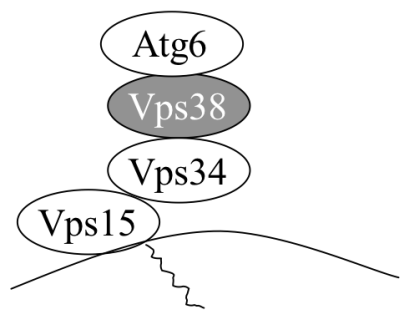

Complex II

Vps pathway

Figure 4: The two Phosphatidylinositol 3-kinase complexes in S. cerevisiae

Both complexes contain Vps15, Vps34 and Atg6 (Vps30), (A) Complex I required for autophagy and the Cvt pathway contains Atg14. (B) Complex II containing Vps38 is involved in Vps/MVB pathway ((Yorimitsu and Klionsky, 2005); modified)

\subsubsection{The pre-autophagosomal structure}

The biogenesis of the double membrane-layered autophagosomes starts with the formation of the so-called phagophore (isolation membrane) at the pre-autophagosomal structure (PAS). The PAS, observed in close proximity to the vacuole, is considered to be the place where autophagosome formation starts and where the autophagic core proteins colocalise. Atg18 is recruited to the PAS in a PtdIns 3-kinase complex I-dependent manner. Mediated by the crosstalk between the different systems, during the initiation of autophagy, Atg8 is transported to the PAS in a most likely PtdIns 3-kinase dependent manner, even in the absence of Atg1 (Guan, 2001; Suzuki, 2001; Strømhaug, 2004; Suzuki, 2007).

\subsubsection{The two conjugation systems}

The two ubiquitin-like conjugation systems are widely conserved in eukaryotic cells and play an essential role for autophagy. In the Atg12-Atg5 conjugation system Atg12 and Atg5 are coupled via an isopeptide bond between a C-terminal glycine of Atg12 and a central lysine of Atg5. Atg12 therefore is activated by the E1-like protein Atg7 that functions like a ubiquitinactivating enzyme. Hence, Atg12 is conjugated through the activity of Atg10 (an E2-like ubiquitin conjugation enzyme) to Atg5. The Atg12-Atg5 conjugate binds to Atg16. Atg16 itself can form a homo-oligomer and therefore mediates the formation of a higher multimeric Atg12-Atg5-Atg16 complex. (Figure 5 (A)). This complex has an E3-like function in the second ubiquitin-like conjugation system, the Atg8-phosphatidylethanolamine (Atg8-PE) 
conjugation system (Figure 5 (B)). In the first step, an arginine at the extreme C-terminus of Atg8 is cleaved off via the cysteine protease Atg4. Thereby, the glycine residue, essential for subsequent reactions, is exposed. Furthermore, $\operatorname{Atg} 8$ is activated by the E1-like Atg7 and transferred to the E2-like Atg3, supported by the Atg12-Atg5-Atg16 complex. Atg3 induces an amide-bound between the C-terminal glycine of Atg8 and an aminogroup of PE, thereby coupling Atg8-PE to the outer and inner membrane of the autophagosome. In contrast to the conjugation of Atg12 to Atg5, the lipidation of Atg8 is reversible. After the formation of the autophagosome, the part of Atg8 at the outer membrane is cleaved off via the cysteine protease Atg4. In contrast, Atg8-PE, bound to the inner surface of the autophagosome, is delivered to the vacuole and degraded. Therefore, GFP-Atg8 is a useful marker protein for the detection of autophagy in degradation assay experiments. Furthermore, GFP-Atg8 has been shown to concentrate at the PAS. Therefore GFP-Atg8 can also be used as marker protein for the visualisation of the PAS in microscopic experiments (Ichimura, 2000; Kirisako, 2000; Ohsumi and Mizushima, 2004; Yorimitsu and Klionsky, 2005; Inoue and Klionsky, 2010).

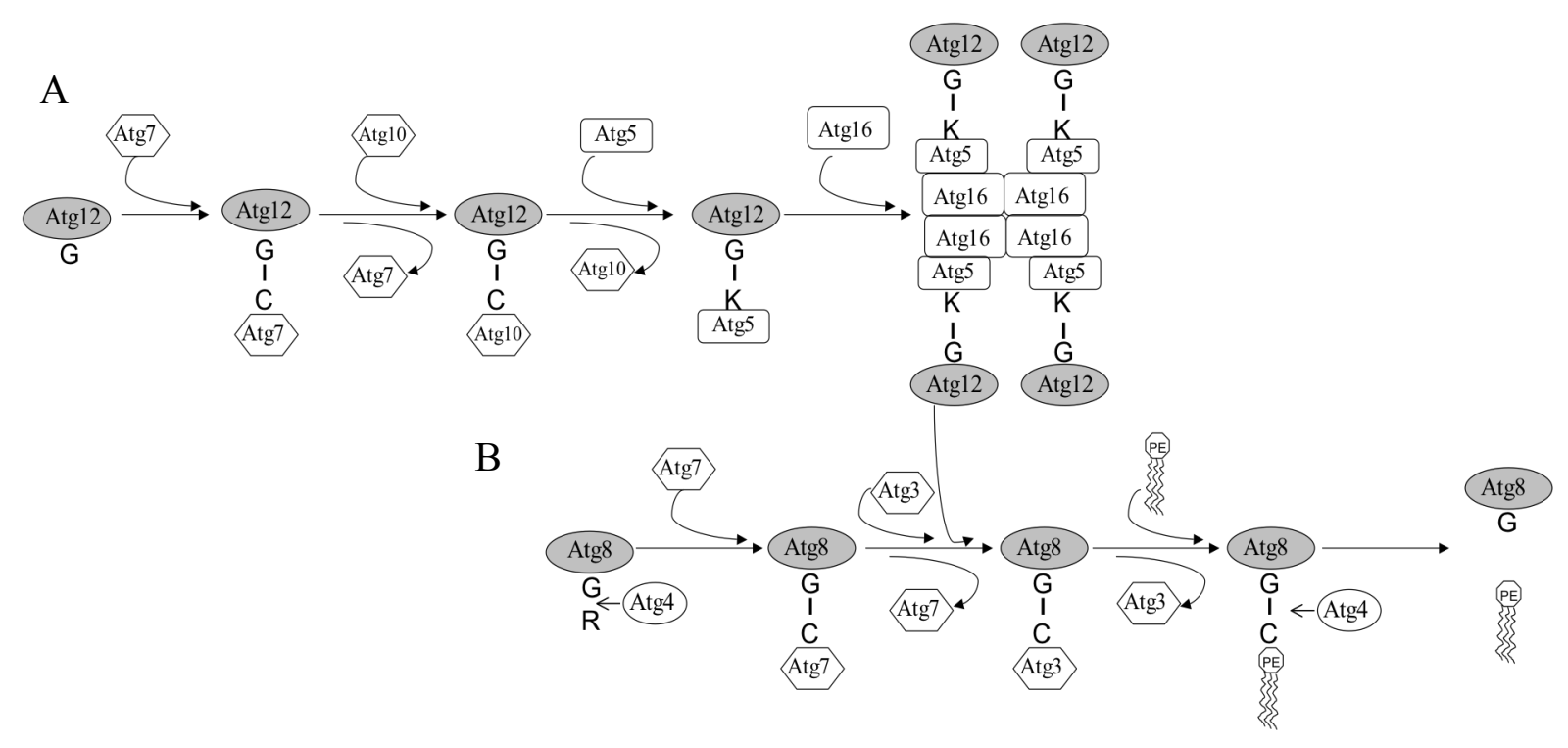

Figure 5: The two conjugation systems

(A) The Atg12-Atg5 conjugation system and (B) the Atg8-PE conjugation system ((Nakatogawa, 2009); modified) 


\subsubsection{Atg9 cycling}

Atg9 is an integral membrane protein, which is recruited to the PAS during assembly. It also localises at a peripheral pool and cycling between both sites has been proposed. A localisation of the peripheral pool at mitochondria, the ER or the Golgi is still under discussion (Ishihara, 2001; Reggiori, 2005; Longatti and Tooze, 2009). Atg9 is thought to mediate the delivery of membranes of unknown origin for the formation of the autophagosome. This suggested lipid flow might be regulated via the Atg1-Atg13 complex and the PtdIns 3-kinase complex I, including a role of Atg2 and Atg18. Atg9 does not affect the formation of the Atg12-Atg5Atg16 complex or Atg8-PE, but is required for their proper localisation at the PAS. The anterograde transport of Atg9 to the PAS has been shown to depend on Atg11, Atg23 and Atg27, whereas Atg1-Atg13 and Atg2-Atg18 are required for the retrograde transport back to the peripheral sites. Due to the cycling of Atg9 to the PAS, also GFP-Atg9 is a useful marker for the visualisation of the PAS in microscopy (Suzuki, 2001; Wang, 2001; Reggiori, 2004a; Reggiori, 2005; Inoue and Klionsky, 2010).

\subsubsection{Microautophagy}

During microautophagy, cytosolic components or organelles are directly engulfed by an invagination of the vacuolar membrane (Farre, 2009). This process topologically reminds to invagination occurring during multivesicular body formation at the endosome (see chapter 2.3), but the mechanism is still unclear. Examples for selective microautophagic processes are pexophagy (see 2.2.4.2) or PMN, the piecemeal microautophagy of the nucleus (see 2.2.4.4).

\subsubsection{Chaperone-mediated autophagy}

The third subtype is chaperone-mediated autophagy, which has only been discovered in mammalian cells but not in $S$. cerevisiae and whose dysregulation is associated with Parkinson's disease (PD) (Yang, 2009). Cytosolic proteins, containing a particular pentapeptide consensus motif (KFERQ), are degraded after the lysosomal import (Cuervo, 2010). 


\subsubsection{Selective autophagy}

\subsubsection{The Cytoplasm to Vacuolar Trafficking pathway}

The Cytoplasm to Vacuolar Trafficking (Cvt) pathway selectively transports two specific cargos to the vacuole: pro-aminopeptidase I (prApe1) and $\alpha$-mannosidase (Ams1). The Cvt pathway requires most of the Atg components and is therefore regarded as a selective autophagic process, which is only active under nutrient rich conditions (Klionsky, 1992; Harding, 1996).

The pro-form of aminopeptidase I (prApe1) is synthesised in the cytosol, where it assembles into the so-called Ape1-complex, an oligomeric structure. The specific receptor protein Atg19 binds to this complex, forming the Cvt-complex, which is recruited at the PAS to a double membrane-layered vesicle, the Cvt vesicle, dependent on Atg11, a protein specific for selective variants of autophagy (Kim, 2001). The Cvt vesicles, which are actually smaller than autophagosomes (140 - $160 \mathrm{~nm}$ in diameter) (Baba, 1997), fuse with the vacuole and a single membrane vesicle is released in the vacuolar lumen, where it is lysed and the prApel is matured to mApe1 (Klionsky, 1992; Scott, 1997).

\subsubsection{Pexophagy}

Pexophagy is the selective autophagic degradation of peroxisomes. Most of the current knowledge of pexophagy resulted from experiments in $P$. pastoris and H. polymorpha. Studies in $H$. polymorpha has shown, that the shift of methanol-grown cells to glucose medium initiates the selective degradation of superfluous peroxisomes in form of macropexophagy, while in $P$. pastoris the same conditions initiate micropexophagy and, however, ethanol induces macropexophagy in both yeasts (Veenhuis, 1983; Tuttle and Dunn, 1995; Sakai, 2006).

$S$. cerevisiae has been shown to adapt the amount of peroxisomes in response to different nutrient sources. This means, that cells, grown on oleic acid as sole carbon source, induce peroxisome biogenesis and a shift to glucose rich or nitrogen limited medium results in the degradation of peroxisomes via pexophagy. Most proteins required for unselective autophagy are also necessary for both micro- and macropexophagy. However, pexophagy requires additional pexophagy-specific proteins (Hutchins, 1999; Farré, 2008). 


\subsubsection{Ribophagy}

Ribophagy is the selective degradation of assembled 40S and 60S ribosomal subunits by autophagy. Interestingly, the ubiquitin protease Ubp3 and its cofactor Bre5 were shown to be needed, indicating the removal of ubiquitin from an unknown substrate. Ubp3 is believed to deubiquitinate Atg19 and is therefore somehow involved in the Cvt pathway (Baxter, 2005), but ribophagy is dependent on the autophagic core machinery and is not affected in $\operatorname{atg} 19 \Delta$ cells (Kraft, 2008).

\subsubsection{Piecemeal Microautophagy of the Nucleus}

Piecemeal microautophagy of the nucleus (PMN) selectively degrades portions of the nucleus at inter-organelle membrane contact sites, which are called the nucleus-vacuole (NV) junctions (Figure $6(\mathrm{I})$ ). These nucleus-vacuole junctions are formed by direct interaction of Vac8 and Nvj1 (Pan, 2000). Furthermore, Osh1, a member of the Osh family in yeast, is targeted to the nucleus-vacuole (NV) junctions through physical interaction with Nvj1. Due to the interaction with NV junctions, Osh1 exhibits a substrate for PMN and is therefore a common marker protein (Millen, 2009). PMN is a selective process and has been shown to depend on the autophagic core proteins (Krick, 2008b). As Atg17, a macroautophagy-specific protein, and Atg11, only required for selective autophagic processes, are involved in PMN, this process is a novel variant of selective autophagy (Roberts, 2003; Krick, 2008b).

Induced by starvation, invagination of the vacuolar membrane sequesters a portion of the nucleus (Figure 6 (II)). After fission of a nuclear membrane derived vesicle (III), a PMN vesicle is released into the vacuole (IV), where it is degraded by vacuolar hydrolases (V) (Krick, 2008b). Up to now, chromosomal DNA and nuclear pore complexes has been shown to be excluded from NV junctions (Pan, 2000; Roberts, 2003), but a specific nuclear cargo is still missing and is a major focus of investigation. 


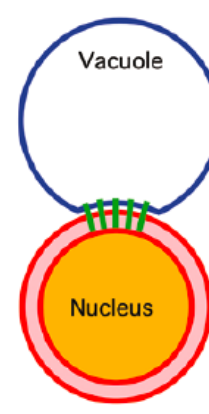

I

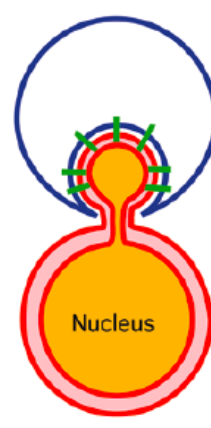

II

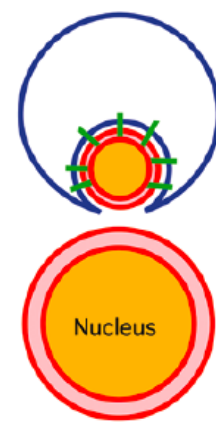

III

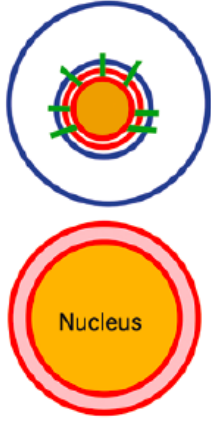

IV

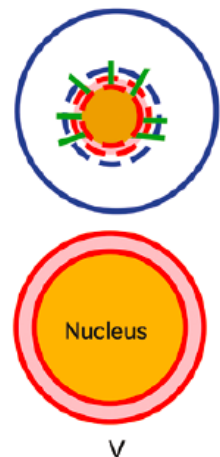

$\mathrm{V}$

Figure 6: Model of piecemeal microautophagy of the nucleus (PMN)

The process of piecemeal microautophagy of the nucleus (PMN) starts with I) the formation of nuclear vacuolar junctions (green), II) then a part of the nucleus is engulfed by vacuolar extentions and III) the nucleus undergoes a fission event. IV) Afterwards a PMN vesicle is released into the vacuole and V) is degraded by vacuolar hydrolases (Krick, 2009).

\subsubsection{Mitophagy}

Mitochondria play a significant role in a lot of important metabolic processes, ranging from energy production to cell death. As a consequence, they are at the same time the major source of cellular reactive oxygen species (ROS), which cause oxidative damage to, for instance, mitochondrial DNA. Therefore a quality control of mitochondria is very important to maintain cellular homeostasis (Nieminen, 2003; Keeble and Gilmore, 2007). In addition to the oxidant defence system (Jamieson, 1998), the selective degradation of mitochondria via autophagy termed mitophagy might fulfil this indispensable role.

Until the last years, mitophagy got more into the focus of research, probably caused by its involvement in many neurodegenerative diseases in humans, like Alzheimer's and Parkinson's disease (PD) (Wallace, 2005; Goldman, 2010; Weber and Reichert, 2010). The ubiquitin ligase Parkin and the mitochondrial kinase PINK1 have been shown to fulfil a crucial role in preventing PD by labelling dysfunctional mitochondria for autophagic degradation (Narendra, 2008).

In S. cerevisiae, recent studies have identified Atg32, a receptor protein exclusively needed for mitophagy. Interaction of Atg32 with Atg8 and a nitrogen starvation induced interaction of Atg8 with Atg11 have been shown. ATG32 deleted cells are no longer able to degrade mitochondria by selective autophagy (Kanki and Klionsky, 2008; Kanki, 2009c; Okamoto, 2009). The exact molecular mechanism of cargo recognition remains unclear. 


\subsubsection{Membrane fusion events in autophagy}

For release of the autophagic body into the vacuole the double membrane-layered autophagosome fuses with the vacuole. Therefore the outer membrane of the autophagosome fuses with the vacuolar membrane. This fusion is mediated by the typical homotypic fusion machinery, including the SNARE proteins Vam3, Vam7, Vti1 and Ykt6, Sec17, Sec18 and Sec19, the Rab protein Ypt7 and members of the class C Vps/HOPS complex and furthermore Ccz1 and Mon1 (Darsow, 1997; Sato, 1998; Fischer von Mollard and Stevens, 1999; Wang, 2003). Even though Sec18 and Vti1 are essential for the fusion of the autophagosome with the vacuole, they are not essential for autophagosome formation, meaning the elongation or sealing of the phagophore (Ishihara, 2001). At today's state of research, the secretory membrane flow from the ER to the Golgi seems to be not directly required for autophagosome formation and there is no evidence for a role of SNAREs in the elongation or sealing of the autophagosomes or Cvt vesicles. Atg proteins might fulfil these essential functions, but the mechanism of autophagosome biogenesis remains unclear (Ishihara, 2001; Reggiori, 2004b; Longatti and Tooze, 2009).

\subsubsection{The $\mathrm{AAA}^{+}$ATPase Cdc48}

Cdc48 (the homologue of p97 in mammals) belongs to the family of $\mathrm{AAA}^{+} \mathrm{ATPases}$ (ATPases associated with various cellular activities) and is a highly conserved protein which is involved in many different cellular processes, including fusion of homotypic membranes, ubiquitin-proteasome-dependent protein degradation, regulation of apoptosis, endoplasmic reticulum (ER)-associated degradation (ERAD) and nuclear envelope reassembly (Latterich, 1995; Ye, 2001; Braun, 2002; Jarosch, 2002; Braun and Zischka, 2008). In all these processes a segregase activity of Cdc48/p97 is believed to be the underlying molecular mechanism. The required energy to extract substrate proteins from protein complexes or lipid membranes is provided by ATP hydrolysis (Braun, 2002; Schuberth and Buchberger, 2008).

To direct the multiple functions of $\mathrm{Cdc} 48 / \mathrm{p} 97$, different cofactors mediate its activity to different cellular pathways. For instance, Cdc48/p97, interacting with the heterodimeric substrate-recruiting cofactor Ufd1/Npl4, affects the degradation of ubiquitinated protein substrates out the ER (Meyer, 2000). Other known cofactors are the members of the UBX protein family (e.g.Ubx1/Shp1), whose UBX domain is a general Cdc48/p97-binding module (Schuberth and Buchberger, 2008). Seven UBX proteins are known for yeast. Three of these UBX proteins, including Ubx1 (Shp1), possess an amino-terminal UBA domain, which binds 
ubiquitinated proteins in vivo (Schuberth, 2004). p47 (the homologue to yeast Shp1) is needed for mitotic Golgi reassembly in mammals, by mediating the binding of p97 to a Golgi tSNARE via an ubiquitinated putative fusion regulator and furthermore regulating the ATPase activity of p97 (Meyer, 1998; Meyer, 2005).

\subsection{Cdc48 and autophagosome biogenesis}

Beside the unknown origin of the autophagic membrane, also the process of autophagosome biogenesis has not been elucidated so far. A recent study has identified the $\mathrm{AAA}^{+} \mathrm{ATPase}$ Cdc48 and its cofactor Shp1/Ubx1 as essential factors for autophagosome biogenesis (Krick, 2010) (Figure 7).

In contrast to p97 (the human Cdc48 homologue) and its cofactor p47 (to human homologue of Ubx1/Shp1), which mediate Golgi reassembly by extraction of an unknown fusion regulator from a protein complex, the $\mathrm{Cdc} 48 / \mathrm{Shp} 1$ complex does not require ubiquitination or the proteasome system for its autophagic function. Atg8 is crucial for control of autophagosomal size/elongation (Nakatogawa, 2007; Xie, 2008a), therefore it has been suggested that the ubiquitin-like Atg8 may replace ubiquitin. This model has been supported by an interaction between Shp1 and the ubiquitin-like protein Atg8. Data of this study contributed to the resulting publication (Krick, 2010).

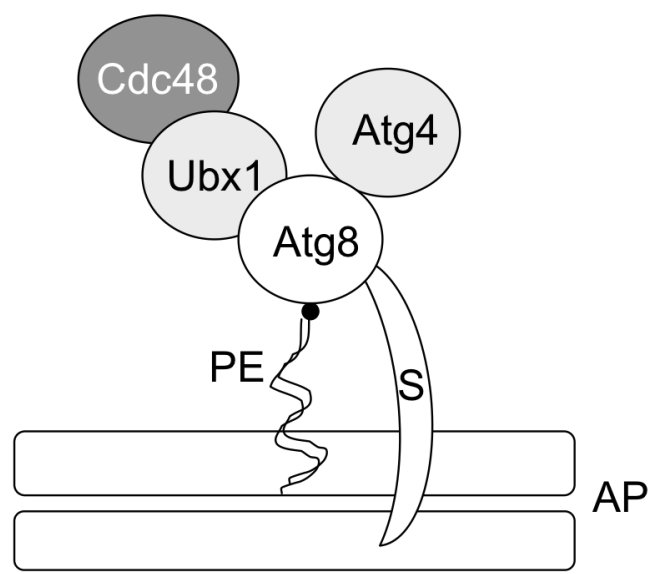

Figure 7: Model of Cdc48-Shp1 complex

The $\mathrm{AAA}^{+}$ATPase Cdc48 and its cofactor Shp1/Ubx1 form a complex needed for autophagosome biogenesis. Shp1 furthermore interacts with the ubiquitin-like protein Atg8, which is anchored via phosphatidylethanolamine (PE) into one layer of the double membrane of the autophagosome (AP). This PE conjugation is cleaved off by the cysteine protease Atg4, which is also needed for the previous lipidation of Atg8. Up to now, an involvement of a SNARE-like protein (S) is unclear. 


\subsection{The ESCRT machinery}

Membrane-bound proteins are actively sorted to different places in the cell through defined processes. Some proteins are recycled back to the plasma membrane or Golgi and others are sorted to the vacuole. Topologic problems of degrading transmembrane proteins in the lumen of the vacuole are solved by the multivesicular body (MVB) pathway, which is mediated by the Vps class E proteins. Monoubiquitin serves as a signal to direct proteins into the MVB pathway. Parts of the endosomal membrane bud into the lumen and form intralumenal vesicles (ILVs). Late endosomes containing these ILVs are termed multivesicular bodies (MVBs). Deletion of one of the Vps class E proteins leads to the formation of an abnormal multicisternal endosome, which lacks internal vesicles and is referred to as class $\mathrm{E}$ compartment. The majority of these class E Vps proteins are members of the endosomal sorting complexes required for transport (ESCRT) (Figure 8). These protein complexes are recruited from the cytoplasm to the endosomal membrane, where they function in the sorting of transmembrane proteins into the MVB pathway or in the formation of MVB vesicles. The ESCRT machinery consists of the five subunits: ESCRT-0, ESCRT-I, ESCRT-II, ESCRT-III and the $\mathrm{AAA}^{+}$ATPase Vps4 (Babst, 2005; Raiborg and Stenmark, 2009). ESCRT-0 consists of Vps27 and Hse1, which both possess ubiquitin interaction motifs (UIMs). These two ubiquitin-binding proteins form a complex, which is recruited to the endosome, mediated by the FYVE domain of Vps27 (Burd and Emr, 1998; Misra and Hurley, 1999). Furthermore Vps27 recruits ESCRT-I, consisting of Vps23, Vps28, Vps37 and Mvb12 (Katzmann, 2001; Katzmann, 2003). This complex also binds to monoubiquitinated proteins, as well as ESCRTII consisting of Vps22, Vps25 and Vps36. Then, the cargo proteins are deubiquitinated by Doa4 and packed into the MVB vesicles by ESCRT-III, which consists of Vps20, Vps32, Vps2 and Vps24 (Amerik, 2000; Babst, 2002). After membrane scission ESCRT-III is disassembled by Vps4 (Babst, 1997). Interestingly, no SNAREs are needed for this membrane scission event (Hurley and Hanson, 2010). 


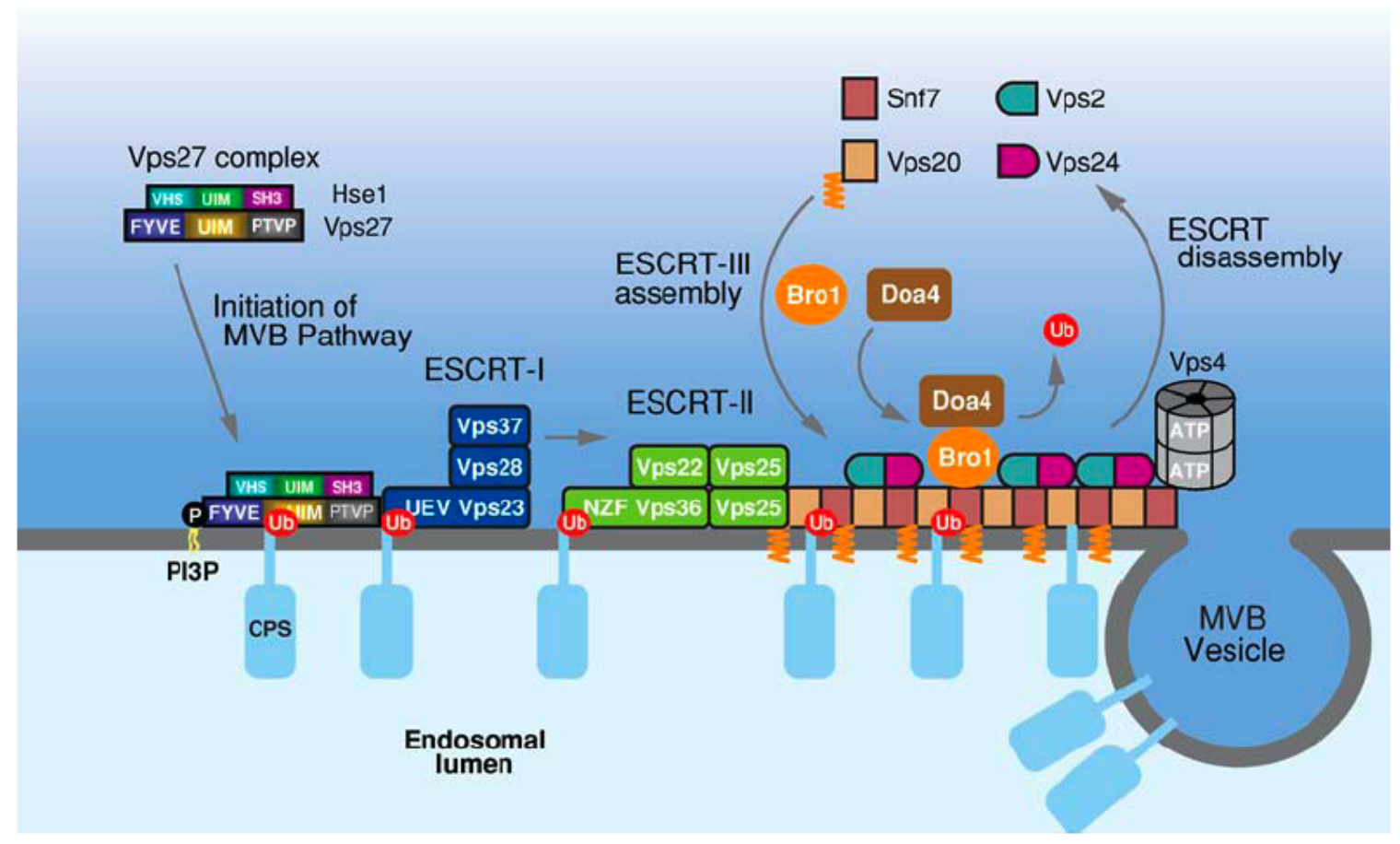

Figure 8: The ESCRT machinery of MVB sorting pathway

(Hurley and Emr, 2006)

\subsubsection{ESCRT and autophagy}

Several reports have analysed the fusion between early and late MVB endosomes with autophagosomes and the resulting generation of amphisomes in mammalian cells, indicating a multistep process (Eskelinen, 2005). Furthermore, in mammalian cells the depletion of ESCRT subunits (e.g. Vps24 (ESCRT-III)) results in an inhibition of the autophagic degradation (Filimonenko, 2007). However, little is known about the potential of MVBs, and the consequence of depleting ESCRT subunits, in autophagic degradation (Fader and Colombo, 2009). Several studies have suggested different roles of the ESCRT machinery. One publication speculates that the inactivation of the ESCRT machinery might prevent the closure of the phagophore, or that the ESCRT machinery might be required for amphisome and autolysosome formation or for lysosome biogenesis (Rusten and Stenmark, 2009). Several reports furthermore discuss the involvement of vacuolar proteins sorting in biogenesis of the vacuolar membrane. In yeast, it is also discussed that Vps class E mutants might impair the fidelity of the vacuolar membrane by defects in vacuolar protein sorting. But the exact influence is still unknown (Piper, 1995; Rieder, 1996; Babst, 1997; Bryant and Stevens, 1998). Nevertheless, in yeast, depletion of one of the Vps class E components, does not lead to a block in autophagy. For instance, the deletion of VPS4, VPS23, VPS27 and VPS28 did 
not effect the maturation of prApel under growing or starvation conditions, or the degradation of GFP-Atg8 under starvation conditions (Reggiori, 2004b; Bremer, 2009). Some deletions lead to a reduced degradation of autophagic material, probably in an indirect way by the impaired vacuolar protein sorting activity and the resulting abnormal vacuolar membrane composition.

\subsection{Aim of the study}

Autophagy is a well-studied process in eukaryotic cells and is involved in numerous diseases, such as neurodegenerative. In yeast, 34 Autophagy related genes (Atg) have already been identified, but molecular details of the molecular mechanism of autophagy still remain unknown.

I. For the identification of autophagy related cooperating or redundant genes, the Synthetic Genetic Array analysis (SGA) should be used. SGA is a powerful high-throughput technique to identify synthetic lethal double deletions, probably reflecting that the gene products impinge on the same essential function. For this purpose, different query strains deleted in autophagic genes should be generated and crossed to the entire deletion library available for $S$. cerevisiae.

II. Different methods to measure selectively bulk autophagy are available, but require a lot of experience, specific deletion strains or are expensive. An aim of this study was to establish a standard method to measure bulk autophagy, using Pgk1-GFP as a marker protein.

III. Until now, the fusion events required for autophagosome biogenesis are poorly understood. No essential SNAREs or autophagy-specific Rab effectors have been identified, therefore a special autophagy fusion machinery might be required. Based on previous results our laboratory, the role of Cdc48 and its adaptor Shp1 in autophagosome formation should be analysed.

IV. In yeast, mitophagy, the selective degradation of mitochondria, is currently not well understood, therefore to monitor mitophagy and to differentiate between the degradation of superfluous or damaged mitochondria in yeast, a specific marker protein for a mitophagy assay and novel methods to induce mitophagy should be established.

V. A specific cargo-recognition in PMN, the piecemeal microautophagy of the nucleus, is indispensable to preserve the integrity of the nucleus. No selective nuclear cargo has been identified so far, therefore the degradation of different nuclear GFP fusion proteins should be analysed. 


\section{Material and Methods}

\subsection{Material}

\subsubsection{Saccharomyces cerevisiae strains}

Table 1: Strains of BY4741 background used in this study

\begin{tabular}{|c|c|c|}
\hline Strain & Genotype & Source \\
\hline BY 4741 & $\begin{array}{l}\text { MATa his } 3 \Delta 1 \text { leu } 2 \Delta 0 \\
\text { met } 15 \Delta 0 \text { ura } 3 \Delta 0\end{array}$ & Euroscarf \\
\hline Y04547 & BY4741 MATa $\operatorname{atg} 1 \Delta:: \mathrm{KAN}$ & Euroscarf \\
\hline Y02098 & BY4741 MATa pep4A::KAN & Euroscarf \\
\hline Y05700 & BY4741 MATa $\operatorname{atg} 184:: \mathrm{KAN}$ & Euroscarf \\
\hline Y02152 & BY4741 MATa $\operatorname{atg} 21 \Delta:: \mathrm{KAN}$ & Euroscarf \\
\hline Y00253 & BY4741 MATa vac84::KAN & Euroscarf \\
\hline Y05876 & BY4741 MATa ygr223c4::KAN & Euroscarf \\
\hline Y03104 & BY4741 MATa atg84::KAN & Euroscarf \\
\hline Y05492 & BY4741 MATa $v p s 4 \Delta:: \mathrm{KAN}$ & Euroscarf \\
\hline Y05381 & BY4741 MATa vps274::KAN & Euroscarf \\
\hline Y02763 & BY4741 MATa vps $284::$ KAN & Euroscarf \\
\hline Y01580 & BY4741 MATa vps324::KAN & Euroscarf \\
\hline Y05269 & BY4741 MATa vps384::KAN & Euroscarf \\
\hline Y03084 & BY4741 MATa shp14::KAN & Euroscarf \\
\hline Y05789 & BY4741 MATa $\operatorname{atg} 154:: \mathrm{KAN}$ & Euroscarf \\
\hline Y07090 & BY4741 MATa $\operatorname{atg} 74:: \mathrm{KAN}$ & Euroscarf \\
\hline Y05382 & BY4741 MATa $\operatorname{atg} 3 \Delta:: \mathrm{KAN}$ & Euroscarf \\
\hline Y05325 & BY4741 MATa vps364::KAN & Euroscarf \\
\hline Y04004 & BY4741 MATa doa4d::KAN & Euroscarf \\
\hline Y06148 & BY4741 MATa $u b p 3 \Delta:: \mathrm{KAN}$ & Euroscarf \\
\hline Y06078 & BY4741 MATa bre5 $4:: \mathrm{KAN}$ & Euroscarf \\
\hline Y02889 & BY4741 MATa nvj14::KAN & Euroscarf \\
\hline Y01989 & BY4741 MATa $\operatorname{atg} 4 \Delta:: \mathrm{KAN}$ & Euroscarf \\
\hline Y05113 & BY4741 MATa uth1 $1:: \mathrm{KAN}$ & Euroscarf \\
\hline Y03682 & BY4741 MATa vac1 $1:: \mathrm{KAN}$ & Euroscarf \\
\hline Y05798 & BY4741 MATa aup $1 \Delta:: \mathrm{KAN}$ & Euroscarf \\
\hline Y02305 & BY4741 MATa atg324::KAN & Euroscarf \\
\hline Y00560 & BY4741 MATa $u b x 2 \Delta:: K A N$ & Euroscarf \\
\hline Y03788 & BY4741 MATa $u b x 3 \Delta:: \mathrm{KAN}$ & Euroscarf \\
\hline Y06200 & BY4741 MATa $u b x 4 \Delta::$ KAN & Euroscarf \\
\hline Y03689 & BY4741 MATa $u b x 5 \Delta:: \mathrm{KAN}$ & Euroscarf \\
\hline Y03888 & BY4741 MATa $u f d 2 \Delta:: \mathrm{KAN}$ & Euroscarf \\
\hline Y05063 & BY4741 MATa $u f d 3 \Delta:: \mathrm{KAN}$ & Euroscarf \\
\hline Y04859 & BY4741 MATa $u f d 4 \Delta:: \mathrm{KAN}$ & Euroscarf \\
\hline Y03341 & BY4741 MATa der1 $1:: \mathrm{KAN}$ & Euroscarf \\
\hline Y05665 & BY4741 MATa otu1 $\triangle::$ KAN & Euroscarf \\
\hline
\end{tabular}




\begin{tabular}{llc}
\hline Strain & \multicolumn{1}{c}{ Genotype } & Source \\
\hline WT Om45- & BY4741 MATa & This study \\
GFP & Om45-GFP::HIS3MX6 & \\
atg14 & BY4741 MATa atg14::KAN & This study \\
Om45-GFP & Om45-GFP::HIS3MX6 & \\
pep44 & BY4741 MATa pep4A::KAN & This study \\
Om45-GFP & Om45-GFP::HIS3MX6 & \\
uth14 & BY4741 MATa uth14::KAN & This study \\
Om45-GFP & Om45-GFP::HIS3MX6 & \\
atg324 & BY4741 MATa atg324::KAN & This study \\
Om45-GFP & Om45-GFP::HIS3MX6 &
\end{tabular}

Table 2: Strains of BY4742 background used in this study

\begin{tabular}{|c|c|c|}
\hline Strain & Genotype & Source \\
\hline BY4742 & $\begin{array}{l}\text { Mat } \alpha \text { his } 3 \Delta 1 ; \text { leu } 2 \Delta 0 ; \text { lys } 2 \Delta 0 \text {; } \\
\text { ura } 3 \Delta 0\end{array}$ & Euroscarf \\
\hline Y11580 & BY4742 MAT $\alpha$ vps324::KAN & Euroscarf \\
\hline Y12098 & BY4742 MAT $\alpha$ рер $4 \Delta:: \mathrm{KAN}$ & Euroscarf \\
\hline Y12152 & BY4742 MAT $\alpha$ atg $21 \Delta:: \mathrm{KAN}$ & Euroscarf \\
\hline Y12580 & BY4742 MAT $\alpha$ vps 254::KAN & Euroscarf \\
\hline Y12763 & BY4742 MAT $\alpha$ vps $284:: \mathrm{KAN}$ & Euroscarf \\
\hline Y13084 & BY4742 MAT $\alpha$ shp $1 \Delta:: \mathrm{KAN}$ & Euroscarf \\
\hline Y13104 & BY4742 MAT $\alpha$ atg $8 \Delta:: \mathrm{KAN}$ & Euroscarf \\
\hline Y13416 & BY4742 MAT $\alpha$ vp $23 \Delta:: \mathrm{KAN}$ & Euroscarf \\
\hline Y14004 & BY4742 MAT $\alpha$ doa4A::KAN & Euroscarf \\
\hline Y14890 & BY4742 MAT $\alpha$ vps $24 \Delta:: \mathrm{KAN}$ & Euroscarf \\
\hline Y15381 & BY4742 MAT $\alpha$ vps $274:: \mathrm{KAN}$ & Euroscarf \\
\hline Y15492 & BY4742 MAT $\alpha$ vps $4 \Delta:: \mathrm{KAN}$ & Euroscarf \\
\hline Y15700 & BY4742 MAT $\alpha$ atg 184::KAN & Euroscarf \\
\hline Y15876 & BY4742 MAT $\alpha$ ygr223c $4:: \mathrm{KAN}$ & Euroscarf \\
\hline Y16078 & BY4742 MAT $\alpha$ bre 54::KAN & Euroscarf \\
\hline Y16148 & BY4742 MAT $\alpha$ ubp3 $3:: \mathrm{KAN}$ & Euroscarf \\
\hline
\end{tabular}

Table 3: Strains of WCG background used in this study

\begin{tabular}{|c|c|c|}
\hline Strain & Genotype & Source \\
\hline WCG4 & $\begin{array}{l}\text { WCG4a MAT } \alpha \text { his3-11,15 } \\
\text { leu2-3,112 ura3 }\end{array}$ & $\begin{array}{l}\text { W. Heinemeyer, } \\
\text { Stuttgart }\end{array}$ \\
\hline WCG4 & $\begin{array}{l}\text { WCG4a MATa his3-11,15 ura3 } \\
\text { leu2-3,112 }\end{array}$ & $\begin{array}{l}\text { W. Heinemeyer, } \\
\text { Stuttgart }\end{array}$ \\
\hline $\operatorname{atg} 21 \Delta$ & WCG4a MAT $\alpha$ mail $1 \Delta:$ KAN & (Barth, 2002) \\
\hline
\end{tabular}




\begin{tabular}{|c|c|c|}
\hline Strain & Genotype & Source \\
\hline $\operatorname{atg} 27 \Delta$ & WCG4a MAT a $\operatorname{atg} 27 \Delta::$ HIS3 & (Bremer, 2009) \\
\hline doa4 4 & WCG4a MAT $\alpha$ doa4A::NAT & This study \\
\hline $\operatorname{shp} 1 \Delta$ & WCG4a MAT $\alpha$ shp1 $1 \Delta:$ NAT & This study \\
\hline YCV10 & WCG4a MAT $\alpha$ atg $4 \Delta \operatorname{atg} 8 \Delta$ & C. Voss \\
\hline YKMW33 & WCG4a MAT $\alpha$ vps $344:: \mathrm{KAN}$ & K. Meiling-Wesse \\
\hline YMTA & WCG4a MATa pep4A::HIS & M. Thumm \\
\hline YUE92 & WCG4a MAT $\alpha$ vps $234:: \mathrm{KAN}$ & U. Epple \\
\hline YUE94 & WCG4a MAT $\alpha$ vps $284:: \mathrm{KAN}$ & U. Epple \\
\hline YYW08 & WCG4a MAT $\alpha$ nvj1A::HIS & (Krick, 2008b) \\
\hline
\end{tabular}

Table 4: RFP-fusion strains used in this study

\begin{tabular}{llc}
\hline Strain & \multicolumn{1}{c}{ Genotype } & Source \\
\hline Sik1-RFP & S288c MATa ura3 leu2 his3 & Falvo \\
& Sik1-RFP::kanMX6 & \\
Sik1-RFP & S288c MATa ura3 leu2 his3 & This study \\
atg14 & Sik1-RFP::kanMX6 atg1A::NAT & \\
Sik1-RFP & S288c MATa ura3 leu2 his3 & This study \\
vac84 & Sik1-RFP::kanMX6 vac84::NAT &
\end{tabular}

Table 5: GFP-fusion strains used in this study

\begin{tabular}{|c|c|c|}
\hline Strain & Genotype & Source \\
\hline $\begin{array}{l}\text { Pgk1-GFP } \\
\text { atglA }\end{array}$ & $\begin{array}{l}\text { Attc201388 Mata ura3 leu2 his3 } \\
\text { Pgk1-GFP::HIS3MX6 } \\
\text { atg14::NAT }\end{array}$ & This study \\
\hline $\begin{array}{l}\text { Sik1-GFP } \\
\operatorname{atg} 1 \Delta\end{array}$ & $\begin{array}{l}\text { Attc } 201388 \text { Mata ura3 leu2 his3 } \\
\text { Sik1-GFP::HIS3MX6 } \\
\text { atg14::NAT }\end{array}$ & This study \\
\hline $\begin{array}{l}\text { Cic1-GFP } \\
\operatorname{atg} 1 \Delta\end{array}$ & $\begin{array}{l}\text { Attc } 201388 \text { Mata ura3 leu2 his3 } \\
\text { Cic1-GFP::HIS3MX6 } \\
\text { atg1 } 1 \text { ::NAT }\end{array}$ & This study \\
\hline YDR227W & $\begin{array}{l}\text { Attc201388 Mat a ura3 leu2 his3 } \\
\text { Sir4-GFP::HIS3MX6 }\end{array}$ & Invitrogen \\
\hline YFL008W & $\begin{array}{l}\text { Attc201388 Mat a ura3 leu2 his3 } \\
\text { Smc1-GFP::HIS3MX6 }\end{array}$ & Invitrogen \\
\hline YJL109C & $\begin{array}{l}\text { Attc201388 Mat a ura3 leu2 his } 3 \\
\text { Utp10-GFP::HIS3MX6 }\end{array}$ & Invitrogen \\
\hline YKL182W & $\begin{array}{l}\text { Attc201388 Mat a ura3 leu2 his } 3 \\
\text { Fas1-GFP::HIS3MX6 }\end{array}$ & Invitrogen \\
\hline YMR219W & $\begin{array}{l}\text { Attc201388 Mat a ura3 leu2 his } 3 \\
\text { Esc1-GFP::HIS3MX6 }\end{array}$ & Invitrogen \\
\hline YPL231W & $\begin{array}{l}\text { Attc201388 Mat a ura3 leu2 his3 } \\
\text { Fas2-GFP::HIS3MX6 }\end{array}$ & Invitrogen \\
\hline YBL045C & $\begin{array}{l}\text { Attc201388 Mat a ura } 3 \text { leu } 2 \text { his } 3 \\
\text { Cor1-GFP::HIS3MX6 }\end{array}$ & Invitrogen \\
\hline
\end{tabular}




\begin{tabular}{|c|c|c|}
\hline Strain & Genotype & Source \\
\hline YBL058W & $\begin{array}{l}\text { Attc201388 Mat a ura3 leu2 his3 } \\
\text { Shp1-GFP::HIS3MX6 }\end{array}$ & Invitrogen \\
\hline YBR009C & $\begin{array}{l}\text { Attc201388 Mat a ura3 leu2 his3 } \\
\text { Hhf1-GFP::HIS3MX6 }\end{array}$ & Invitrogen \\
\hline YBR283C & $\begin{array}{l}\text { Attc201388 Mat a ura3 leu2 his3 } \\
\text { Ssh1-GFP*HIS3MX6 }\end{array}$ & Invitrogen \\
\hline & Attc201388 Mat a ura3 leu2 his3 & Invitrogen \\
\hline YCR012W & $\begin{array}{l}\text { Pgk1-GFP::HIS3MX6 } \\
\text { Attc201388 Mat a ura3 leu2 his3 }\end{array}$ & \\
\hline YCR084C & Tup1-GFP::HIS3MX6 & Invitrogen \\
\hline YDL089W & $\begin{array}{l}\text { Attc } 201388 \text { Mat a ura3 leu } 2 \text { his } 3 \\
\text { YDL089W -GFP::HIS3MX6 }\end{array}$ & Invitrogen \\
\hline & Attc201388 Mat a ura3 leu2 his3 & Invitrogen \\
\hline YDL126C & Cdc48-GFP::HIS3MX6 & inviltogen \\
\hline YDL185W & $\begin{array}{l}\text { Attc201388 Mat a ura3 leu2 his3 } \\
\text { Vma1/Tfp1-GFP::HIS3MX6 }\end{array}$ & Invitrogen \\
\hline YDL213C & $\begin{array}{l}\text { Attc201388 Mat a ura3 leu2 his3 } \\
\text { Nop6-GFP::HIS3MX6 }\end{array}$ & Invitrogen \\
\hline YDR091C & $\begin{array}{l}\text { Attc201388 Mat a ura3 leu2 his3 } \\
\text { Rli1-GFP::HIS3MX6 }\end{array}$ & Invitrogen \\
\hline DR120C & $\begin{array}{l}\text { Attc201388 Mat a ura3 leu2 his3 } \\
\text { Trm1-GFP. HIS } 3 \text { MX6 }\end{array}$ & Invitrogen \\
\hline 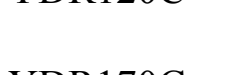 & Attc201388 Mat a ura3 leu2 his3 & \\
\hline YDR170C & Sec7-GFP::HIS3MX6 & Invitrogen \\
\hline YDR233C & $\begin{array}{l}\text { Attc201388 Mat a ura3 leu2 his3 } \\
\text { Rtn1-GFP::HIS3MX6 }\end{array}$ & Invitrogen \\
\hline YDR298C & $\begin{array}{l}\text { Attc201388 Mata ura3 leu2 his3 } \\
\text { Atp5-GFP::HIS3MX6 }\end{array}$ & Invitrogen \\
\hline YDR320C & $\begin{array}{l}\text { Attc201388 Mat a ura3 leu2 his3 } \\
\text { Aux1/Swa2-GFP::HIS3MX6 }\end{array}$ & Invitrogen \\
\hline YDR328C & $\begin{array}{l}\text { Attc201388 Mat a ura3 leu2 his3 } \\
\text { Skp1-GFP::HIS3MX6 }\end{array}$ & Invitrogen \\
\hline YDR398W & $\begin{array}{l}\text { Attc201388 Mat a ura3 leu2 his3 } \\
\text { Utp5-GFP::HIS3MX6 }\end{array}$ & Invitrogen \\
\hline YDR448W & $\begin{array}{l}\text { Attc201388 Mat a ura3 leu2 his3 } \\
\text { Ada2-GFP::HIS3MX6 }\end{array}$ & Invitrogen \\
\hline YDR525W-A & $\begin{array}{l}\text { Attc201388 Mat a ura3 leu2 his3 } \\
\text { Sna2-GFP::HIS3MX6 }\end{array}$ & Invitrogen \\
\hline YEL013W & $\begin{array}{l}\text { Attc201388 Mat a ura3 leu2 his3 } \\
\text { vac8-GFP::HIS3MX6 }\end{array}$ & Invitrogen \\
\hline YEL024W & $\begin{array}{l}\text { Attc201388 Mat a ura3 leu2 his3 } \\
\text { Rip1-GFP::HIS3MX6 }\end{array}$ & Invitrogen \\
\hline YFR050C & $\begin{array}{l}\text { Attc201388 Mat a ura3 leu2 his3 } \\
\text { Pre4-GFP::HIS3MX6 }\end{array}$ & Invitrogen \\
\hline YFR052W & $\begin{array}{l}\text { Attc201388 Mat a ura3 leu2 his3 } \\
\text { Rpn12-GFP::HIS3MX6 }\end{array}$ & Invitrogen \\
\hline YGL038C & $\begin{array}{l}\text { Attc } 201388 \text { Mat a ura3 leu2 his3 } \\
\text { Och1-GFP::HIS3MX6 }\end{array}$ & Invitrogen \\
\hline YGL073W & $\begin{array}{l}\text { Attc201388 Mat a ura3 leu2 his } 3 \\
\text { Hsf1-GFP::HIS3MX6 }\end{array}$ & Invitrogen \\
\hline
\end{tabular}




\begin{tabular}{|c|c|c|}
\hline Strain & Genotype & Source \\
\hline YGL078C & $\begin{array}{l}\text { Attc201388 Mat a ura3 leu2 his3 } \\
\text { Dbp3-GFP::HIS3MX6 }\end{array}$ & Invitrogen \\
\hline YGL122C & $\begin{array}{l}\text { Attc201388 Mat a ura3 leu2 his3 } \\
\text { Nab2-GFP::HIS3MX6 }\end{array}$ & Invitrogen \\
\hline YGL153W & $\begin{array}{l}\text { Attc201388 Mat a ura3 leu2 his3 } \\
\text { Pex4-GFP::HIS3MX6 }\end{array}$ & Invitrogen \\
\hline VCI 172W & Attc201388 Mat a ura3 leu 2 his 3 & Invitrogen \\
\hline YGL1/2W & $\begin{array}{l}\text { Nup49-GFP::HIS3MX6 } \\
\text { Attc201388 Mat a ura3 leu2 his3 }\end{array}$ & \\
\hline YGL187C & Cox4-GFP::HIS3MX6 & Invitrogen \\
\hline YGL194C & $\begin{array}{l}\text { Attc201388 Mat a ura3 leu2 his3 } \\
\text { Hos2-GFP::HIS3MX6 }\end{array}$ & Invitrogen \\
\hline YGR095C & $\begin{array}{l}\text { Attc201388 Mat a ura3 leu2 his3 } \\
\text { Rrp46-GFP::HIS3MX6 }\end{array}$ & Invitrogen \\
\hline YGR112W & $\begin{array}{l}\text { Attc201388 Mat a ura3 leu2 his3 } \\
\text { Shy1-GFP::HIS3MX6 }\end{array}$ & Invitrogen \\
\hline YGR140W & $\begin{array}{l}\text { Attc201388 Mat a ura3 leu2 his3 } \\
\text { ndc10/cbf2-GFP::HIS3MX6 }\end{array}$ & Invitrogen \\
\hline YGR159C & $\begin{array}{l}\text { Attc201388 Mat a ura3 leu2 his3 } \\
\text { Nsr1-GFP::HIS3MX6 }\end{array}$ & Invitrogen \\
\hline YGR202C & $\begin{array}{l}\text { Attc } 201388 \text { Mat a ura3 leu2 his3 } \\
\text { Pct1-GFP::HIS3MX6 }\end{array}$ & Invitrogen \\
\hline YGR252W & $\begin{array}{l}\text { Attc201388 Mat a ura3 leu2 his3 } \\
\text { Gcn5-GFP::HIS3MX6 }\end{array}$ & Invitrogen \\
\hline YHL020C & $\begin{array}{l}\text { Attc201388 Mat a ura3 leu2 his3 } \\
\text { Opi1-GFP::HIS3MX6 }\end{array}$ & Invitrogen \\
\hline YHR052W & $\begin{array}{l}\text { Attc201388 Mat a ura3 leu2 his3 } \\
\text { Cic1-GFP::HIS3MX6 }\end{array}$ & Invitrogen \\
\hline YHR195W & $\begin{array}{l}\text { Attc201388 Mat a ura3 leu2 his3 } \\
\text { Nvj1-GFP::HIS3MX6 }\end{array}$ & Invitrogen \\
\hline YIL026C & $\begin{array}{l}\text { Attc201388 Mat a ura3 leu2 his } 3 \\
\text { Irr1-GFP::HIS3MX6 }\end{array}$ & Invitrogen \\
\hline YIL048W & $\begin{array}{l}\text { Attc201388 Mat a ura3 leu2 his3 } \\
\text { Neo1-GFP::HIS3MX6 }\end{array}$ & Invitrogen \\
\hline YIL090W & $\begin{array}{l}\text { Attc201388 Mat a ura3 leu2 his3 } \\
\text { Ice2-GFP::HIS3MX6 }\end{array}$ & Invitrogen \\
\hline YIL136W & $\begin{array}{l}\text { Attc201388 Mat a ura3 leu2 his3 } \\
\text { Om45-GFP::HIS3MX6 }\end{array}$ & Invitrogen \\
\hline YJL001W & $\begin{array}{l}\text { Attc201388 Mat a ura3 leu2 his3 } \\
\text { Pre3-GFP::HIS3MX6 }\end{array}$ & Invitrogen \\
\hline YJL173C & $\begin{array}{l}\text { Attc201388 Mat a ura3 leu2 his3 } \\
\text { Rfa3-GFP::HIS3MX6 }\end{array}$ & Invitrogen \\
\hline YJR060W & $\begin{array}{l}\text { Attc201388 Mat a ura3 leu2 his } 3 \\
\text { Cbf1-GFP::HIS3MX6 }\end{array}$ & Invitrogen \\
\hline YKL049C & $\begin{array}{l}\text { Attc201388 Mat a ura3 leu2 his3 } \\
\text { Cse4-GFP::HIS3MX6 }\end{array}$ & Invitrogen \\
\hline YKR092C & $\begin{array}{l}\text { Attc201388 Mat a ura3 leu2 his3 } \\
\text { Srp40-GFP::HIS3MX6 }\end{array}$ & Invitrogen \\
\hline YKR095W & $\begin{array}{l}\text { Attc201388 Mat a ura3 leu2 his3 } \\
\text { Mlp1-GFP::HIS3MX6 }\end{array}$ & Invitrogen \\
\hline
\end{tabular}




\begin{tabular}{|c|c|c|}
\hline Strain & Genotype & Source \\
\hline YLR002C & $\begin{array}{l}\text { Attc201388 Mat a ura3 leu2 his3 } \\
\text { Noc3-GFP::HIS3MX6 }\end{array}$ & Invitrogen \\
\hline YLR196W & $\begin{array}{l}\text { Attc201388 Mat a ura3 leu2 his3 } \\
\text { Pwp1-GFP::HIS3MX6 }\end{array}$ & Invitrogen \\
\hline YLR197W & $\begin{array}{l}\text { Attc201388 Mat a ura3 leu2 his } 3 \\
\text { Sik1-GFP. HIS } 3 \text { MX6 }\end{array}$ & Invitrogen \\
\hline & Attc201388 Mat a ura3 leu2 his3 & Invitrogen \\
\hline YLR347C & $\begin{array}{l}\text { Kap95-GFP::HIS3MX6 } \\
\text { Attc201388 Mat a ura3 leu2 his3 }\end{array}$ & \\
\hline YML034W & Src1-GFP::HIS3MX6 & Invitrogen \\
\hline YML075C & $\begin{array}{l}\text { Attc201388 Mat a ura3 leu2 his3 } \\
\text { Hmg1-GFP::HIS3MX6 }\end{array}$ & Invitrogen \\
\hline YMR243C & $\begin{array}{l}\text { Attc201388 Mat a ura3 leu2 his3 } \\
\text { Zrc1-GFP::HIS3MX6 }\end{array}$ & Invitrogen \\
\hline & Attc201388 Mat a ura3 leu2 his3 & Invitrogen \\
\hline YMR284W & Yku70-GFP::HIS3MX6 & IIIVIIIOgen \\
\hline YNL030W & $\begin{array}{l}\text { Attc201388 Mat a ura3 leu2 his3 } \\
\text { Hhf2-GFP::HIS3MX6 }\end{array}$ & Invitrogen \\
\hline YNL037C & $\begin{array}{l}\text { Attc201388 Mat a ura3 leu2 his3 } \\
\text { Idh1-GFP::HIS3MX6 }\end{array}$ & Invitrogen \\
\hline YNL037c & $\begin{array}{l}\text { Attc201388 Mat a ura3 leu2 his3 } \\
\text { Idh1-GFP::HIS3MX6 }\end{array}$ & Invitrogen \\
\hline YNL330C & $\begin{array}{l}\text { Attc201388 Mat a ura3 leu2 his3 } \\
\text { Rpd3-GFP::HIS3MX6 }\end{array}$ & Invitrogen \\
\hline YOL006C & $\begin{array}{l}\text { Attc201388 Mat a ura3 leu } 2 \text { his } 3 \\
\text { TOP1-GFP::HIS3MX6 }\end{array}$ & Invitrogen \\
\hline YOL010W & $\begin{array}{l}\text { Attc201388 Mat a ura3 leu2 his3 } \\
\text { Rcl1-GFP::HIS3MX6 }\end{array}$ & Invitrogen \\
\hline YOL072W & $\begin{array}{l}\text { Attc201388 Mat a ura3 leu2 his3 } \\
\text { Thp1-GFP::HIS3MX6 }\end{array}$ & Invitrogen \\
\hline YOL144W & $\begin{array}{l}\text { Attc201388 Mat a ura3 leu2 his3 } \\
\text { Nop8-GFP::HIS3MX6 }\end{array}$ & Invitrogen \\
\hline YOR230W & $\begin{array}{l}\text { Attc201388 Mat a ura3 leu2 his3 } \\
\text { Wtm1-GFP::HIS3MX6 }\end{array}$ & Invitrogen \\
\hline YOR254C & $\begin{array}{l}\text { Attc201388 Mat a ura3 leu2 his3 } \\
\text { Sec63-GFP::HIS3MX6 }\end{array}$ & Invitrogen \\
\hline YOR270C & $\begin{array}{l}\text { Attc } 201388 \text { Mat a ura3 leu } 2 \text { his } 3 \\
\text { Vph1-GFP::HIS3MX6 }\end{array}$ & Invitrogen \\
\hline YOR293W & $\begin{array}{l}\text { Attc201388 Mat a ura3 leu2 his3 } \\
\text { Rps10a -GFP::HIS3MX6 }\end{array}$ & Invitrogen \\
\hline YOR310C & $\begin{array}{l}\text { Attc201388 Mat a ura3 leu2 his3 } \\
\text { Nop5/Nop58-GFP::HIS3MX6 }\end{array}$ & Invitrogen \\
\hline YPL018W & $\begin{array}{l}\text { Attc201388 Mat a ura3 leu2 his3 } \\
\text { Ctf19-GFP::HIS3MX6 }\end{array}$ & Invitrogen \\
\hline YPL128C & $\begin{array}{l}\text { Attc201388 Mat a ura3 leu2 his3 } \\
\text { Tbf1-GFP::HIS3MX6 }\end{array}$ & Invitrogen \\
\hline YPL139C & $\begin{array}{l}\text { Attc201388 Mat a ura3 leu2 his3 } \\
\text { Ume1-GFP::HIS3MX6 }\end{array}$ & Invitrogen \\
\hline YPL212C & $\begin{array}{l}\text { Attc201388 Mat a ura3 leu2 his } 3 \\
\text { Pus1-GFP::HIS3MX6 }\end{array}$ & Invitrogen \\
\hline
\end{tabular}




\begin{tabular}{lll}
\hline Strain & \multicolumn{1}{c}{ Genotype } & Source \\
\hline \multirow{2}{*}{ YPR028W } & Attc201388 Mat a ura3 leu2 his3 & Invitrogen \\
& Yop1-GFP::HIS3MX6
\end{tabular}

Table 6: Strains used for SGA

\begin{tabular}{|c|c|c|}
\hline Strain & Genotype & Source \\
\hline Y8205 & $\begin{array}{l}\text { MAT } \alpha \text { can } 1 \Delta:: \text { STE2pr-his5 } \\
\text { lyp } 1 \Delta:: \text { STE3pr-LEU2 } \\
\text { ura } 3 \Delta 0 \text { leu } 2 \Delta 0 \text { his } 3 \Delta 1 \text { met } 15 \Delta\end{array}$ & Boone lab \\
\hline $\begin{array}{l}\text { BY4741 } \\
\text { deletion } \\
\text { library }\end{array}$ & $\begin{array}{l}\text { MAT a } x_{(D M A)} \Delta:: \mathrm{KAN} \\
\text { his } 3 \Delta \text { l leu } 2 \Delta 0 \text { met } 15 \Delta 0 \text { ura } 3 \Delta 0\end{array}$ & Euroscarf \\
\hline
\end{tabular}

Table 7: Strains for mating type determination

\begin{tabular}{llc}
\hline Strain & \multicolumn{1}{c}{ Genotype } & Source \\
\hline YR312 & $\begin{array}{l}\text { Mat a his1-123 test strain for } \\
\text { mating type determination }\end{array}$ & $\begin{array}{l}\text { H. Rudolph, } \\
\text { Stuttgart }\end{array}$ \\
YR320 & $\begin{array}{l}\text { Mat } \alpha \text { his 1-123 test strain for } \\
\text { mating type determination }\end{array}$ & $\begin{array}{l}\text { H. Rudolph, } \\
\text { Stuttgart }\end{array}$
\end{tabular}

\subsubsection{Escherichia coli}

Table 8: Escherichia coli strains used in this study

\begin{tabular}{|c|c|c|}
\hline Name & Genotype & Source \\
\hline DH5 $\alpha$ & $\begin{array}{l}\mathrm{F}^{\prime}(\Phi 80(\text { LlacZ) M15) } \Delta \\
(\text { lacZYA-argF }) \text { U169 recA1 } \\
\text { endA1 hsdR17 } \mathrm{rk}_{\mathrm{k}} \mathrm{m}_{\mathrm{k}}+ \\
\text { supE44 thi-1 gyrA relA }\end{array}$ & $\begin{array}{l}\text { (Hanahan, } \\
\text { 1983) }\end{array}$ \\
\hline BL21 (DE3) pLysS & $\begin{array}{l}\mathrm{F}^{\prime} d c m \text { ompT hsdSB (rB-, } \\
m B-) \text { gal } \lambda(D E 3) ; p L y s S \\
(\text { CamR) }\end{array}$ & Stratagene \\
\hline BL21 (DE3)-RIPL & $\begin{array}{l}\text { F' ompT hsdS }(r B-, m B-) \\
d c m+\text { Tet }+ \text { Gal } \lambda(D E 3) \text { endA } \\
\text { Hte; argU prol CamR; argU } \\
\text { ileY leuW Strep/SpecR }\end{array}$ & Stratagene \\
\hline
\end{tabular}


3.1.3 Plasmids

Table 9: Plasmids used in this study

\begin{tabular}{|c|c|c|}
\hline Name & Genotype & Source \\
\hline GFP-Atg8 & $\begin{array}{l}\text { pRS316 CEN6 URA3 } \\
\text { GFP-Atg8 }\end{array}$ & (Suzuki, 2001) \\
\hline Pgk1-GFP & $\begin{array}{l}\text { pRS316 CEN6 URA3 } \\
\text { Pgk1-GFP }\end{array}$ & (Welter, 2010) \\
\hline mito-GFP & pRS316-Su9-mtDHFR-GFP & This study \\
\hline GST-shp1 & pGEX-4T3-Shp1 & This study \\
\hline GST-shp1-UBX & pGEX-4T3-Shp1-UBX & This study \\
\hline GST-shp1-UBA & pGEX-4T3-Shp1-UBA & This study \\
\hline NLSmcherry & $\begin{array}{l}\text { pYX242 } 2 \mu \text { LEU2 TPI } \\
\text { Nab2NLS-2m cherry }\end{array}$ & B. Timney and M. Rout \\
\hline RFP-Atg8 & $\begin{array}{l}\text { pRS313 Cen6 HIS3 } \\
\text { RFP-Atg8 }\end{array}$ & S. Bremer \\
\hline GFP-Atg9 & $\begin{array}{l}\text { CEN6 URA3 MET25 } \\
\text { GFP-Atg9 }\end{array}$ & (Meiling-Wesse, 2004) \\
\hline pUG35 & pUG35 CEN6 URA3 & J. H. Hegemann \\
\hline pRS316 & pRS316 CEN6 URA3 & $\begin{array}{c}\text { (Sikorski and Hieter, } \\
1989 \text { ) }\end{array}$ \\
\hline GFP-Atg8-FG & $\begin{array}{l}\text { pRS316 CEN6 URA3 } \\
\text { GFP-Atg8-FG }\end{array}$ & $\begin{array}{c}\text { (Suzuki, 2001) } \\
\text { (Kirisako, 2000) }\end{array}$ \\
\hline pOm45-GFP & $\begin{array}{l}\text { pRS316 CEN6 URA3 } \\
\text { Om45-GFP }\end{array}$ & this study \\
\hline pIdh1-GFP & $\begin{array}{l}\text { pRS316 CEN6 URA3 } \\
\text { Idh1-GFP }\end{array}$ & this study \\
\hline GFP-Osh1 & $\begin{array}{l}\text { pRS416 CEN6 URA3 } \\
\text { GFP-Osh1 }\end{array}$ & (Loewen, 2003) \\
\hline pFA6-natNT2 & natNT2 & (Janke, 2004) \\
\hline PFA6a-HIS3MX6 & HIS3MX6 & (Longtine, 1998) \\
\hline $\begin{array}{l}\text { PFA6a-kanMX6 } \\
\text { pHO }\end{array}$ & $\begin{array}{l}\text { kanMX6 } \\
\text { for mating type switch; pGal }\end{array}$ & $\begin{array}{c}\text { (Longtine, 1998) } \\
\text { unknown }\end{array}$ \\
\hline
\end{tabular}

\subsubsection{Oligonucleotides}

Table 10: Oligonucleotides used in this study

acquired by purchase from: www.eurofins.com

\begin{tabular}{ll}
\hline Name & \multicolumn{1}{c}{ Sequence (5'-3') } \\
\hline shp1s::natNT2 & \\
shp1_S1f: & ATATATAAGAAACGTCGGTAGCACAACAATTAACTCATTATTT \\
& AGGTATGCGTACGCTGCAGGTCGAC \\
shp1_S2r: & ATATTAAGTTGAAGTCTTTTCCCGTTCTGTTTTTGTATATTTA \\
& TGCTCAATCGATGAATTCGAGCTCG
\end{tabular}




\begin{tabular}{|c|c|}
\hline Name & Sequence (5'-3') \\
\hline \multicolumn{2}{|l|}{$\operatorname{atg1\Delta ::natNT2}$} \\
\hline atg1_ko_fw & $\begin{array}{l}\text { CCCCATATTTTCAAATCTCTTTTACAACACCAGACGAGAAATT } \\
\text { AAGAAAATG CGTACGCT GCAGGTCGAC }\end{array}$ \\
\hline atg1_ko_rev & $\begin{array}{l}\text { GCAGGTCATTTGTACTTAATAAGAAAACCATATTATGCATCAC } \\
\text { TTAATCGATGAATTCGAGCTCG }\end{array}$ \\
\hline \multicolumn{2}{|c|}{ doa4 $\Delta::$ natNT2 } \\
\hline Doa4_ko_frw & $\begin{array}{l}\text { GTGCACGCTTCCAAAGTTTTTTTTACTATTTGATACATGCTTAA } \\
\text { GTTATGCGTACGCT GCAGGTCGAC }\end{array}$ \\
\hline Doa4_ko_rev & $\begin{array}{l}\text { CGGAAAAAAAGTGTATAGACAACGGTTTTCAGTTATTTATTCA } \\
\text { AATGAATCAATCGATGAA TTCGAGCTCG }\end{array}$ \\
\hline \multicolumn{2}{|c|}{ vac8 $\Delta::$ natNT2 } \\
\hline Vac8-his_fw & $\begin{array}{l}\text { CTATAAGGGTGTTCTTTCTTCTGTACTATATATACATTTGCAAC } \\
\text { TATGCGGATCCCGGGTTAATTAA }\end{array}$ \\
\hline Vac8-his rev & $\begin{array}{l}\text { AAAATTTTGATAAAAATTATAATGCCTAGTCCCGCTTTTGAAG } \\
\text { AAAATCAGAATTCGAGCTCGTTTAAAC }\end{array}$ \\
\hline
\end{tabular}

pAG25

Nat_frw

Nat_rev

AGC TTG CCT TGT CCC CGC CG

TCG ACA CTG GAT GGC GGC GT

control primer

NAT_1r

CCTCCATGTCGCTGGCCG

1f Shp1 XbaI

atg1_1_fw

TCCGCCTATCTAGACATAACTTATTTACATAGTGCC

atg1_2_rev

GGGGAAACAGAGAACAGTAC

$\operatorname{atg} 13 \mathrm{rev}$

CCCATCCAGGTATAAAAGAGC

Doa 4 1_frw

GCCTTTGCGGCCAGAGATTCC

Doa4_2_rev

CTTCGGTAGT ATAGGGCAG

Doa4_3_rev

GATGGAAATA ATGATATGCC

Vac8_K1fw:

CACCAGAATG TCCAACAGGA C

CGGTAAGGAAGTGGGATCTGTAGCG

Vac8_K2rev:

Vac8_K3rev:

CCGAACAACCCCGGTTCGAATCCG

Vac8_K4fw

Vac8 K5rev

CGAGCTTGGATTGTTGTTGCTCCCC

CTTTTCGTCACTGACGGGTCCCTGC

CCCACCATCAGAGGACGAAGAGAG

Idh1-GFP f

GAATTGGAGCTCCACCGCGGTGGCGGCCGCTCTAGAAC

Idh1TAGTGGATCC GCTGTAGTTGATGTGGTTGGG

GFP/OM45CTCGAGGTCGACGGTATCGATAAGCTTGATATCGAATTCCTG

GFP $\mathrm{r}$

OM45-GFP f

CAGGCAACCTGACCTACAGGAAAG

Sequence primer

Prs316seq

OM45seq1

GAATTGGAGCTCCACCGCGGTGGCGGCCGCTCTAGAACTAGT GGATCCGTATAGCACATCCAGGAATGG

OM45seq2

GATGTGCTGCAAGGCGATTAAG

OM45seq3

GTCGAGTTCCTTGAAGAGGAG

IDH1seq1

GCAAAGCATCTTCAATTGG

IDH1seq2

GCCAGAGGTTTAGAAGGATG

GATTTCTTTACTCTACCGTAG

IDH1seq3

GAAGGGTGTCAAGACTAG

GTTCCAGACATGTTGGTTTAG 


\begin{tabular}{|c|c|}
\hline Name & Sequence (5'-3') \\
\hline \multicolumn{2}{|l|}{ mt-DHFR } \\
\hline Su9 for & TCTAGAGGATCCATGGCCTCCACTCGTGTCCTCG \\
\hline Su9 rev & GTCGACAAGCTTGTCTTTCTTCTCGTAGACTTCAAAC \\
\hline \multicolumn{2}{|l|}{$\begin{array}{l}\text { GST-shp1- } \\
\text { construct }\end{array}$} \\
\hline GST-SHP1-Eco & AGTAGGAATGAATTCGATGGCGGAAATACCTGATGAAAC \\
\hline GST-SHP1-Xho & AAGTAGGAATCTCGAGTCATGCCCATCTTTGCACGAC \\
\hline GST-SHP1 $1^{-U B A}-$ & AGTAGGAATGAATTCGCAAAAGGATAGAAGAGAGGAAGC \\
\hline $\begin{array}{l}\text { GST-SHP1- } \\
\text { UBX-Xho } \\
\text { Sequence }\end{array}$ & AAGTAGGAATCTCGAGTCAGTCCGGCATGGGTTGTTCCTG \\
\hline Shp1 Seq1f & ССТТСТССАССААСТСТТСG \\
\hline Shp1 Seq1r & CGAAGAGTTGGTGGAGAAGG \\
\hline Shp1 Seq2f & GATTCCGTGATGACGAAGAC \\
\hline Shp1 Seq2r & GTCTTCGTCATCACGGAATC \\
\hline Shp1 Seq3f & GCCAAAGACTAGGATCTCC \\
\hline Shp1 Seq3r & GGAGATCCTAGTCTTTGGC \\
\hline \multicolumn{2}{|l|}{$\begin{array}{l}\text { Chromosomal } \\
\text { integration }\end{array}$} \\
\hline cOM45-GFP for & $\begin{array}{l}\text { ATGGAATGATAAGGGTGATGGTAAATTCTGGAGCTCGAAAAA } \\
\text { GGACGGTCGACGGATCCCCGGGTT }\end{array}$ \\
\hline cOM45-GFP rev & $\begin{array}{l}\text { GTATATATGTTATGCGGGAACCAACCCTTTACAATTAGCTATC } \\
\text { TAATCGATGAATTCGAGCTCGTT }\end{array}$ \\
\hline cOM45_b_fw & $\begin{array}{l}\text { TGATAAGGGTGATGGTAAAATCTGGAGCTCGAAAAAGAACCG } \\
\text { GATCCCCGGGTTAATTAA }\end{array}$ \\
\hline cOM45_b_rev & $\begin{array}{l}\text { GAGAAACATGTGAATATGTATATATGTTATGCGGGAACCAGA } \\
\text { ATTCGAGCTCGTTTAAAC }\end{array}$ \\
\hline IDH1-GFP for & $\begin{array}{l}\text { CTACTACTGACTTCACGAATGAAATCATCAACAAATTATCTAC } \\
\text { CATGGGTCGACGGATCCCCGGGTT }\end{array}$ \\
\hline IDH1-GFP rev & $\begin{array}{l}\text { TGAACACACTTAAGTTGCAGAACAAAAAAAAGGGGAATTG } \\
\text { TTTTCATCGATGAATTCGAGCTCGTT }\end{array}$ \\
\hline control primer & \\
\hline K-OM45 & CGCGCTTCTGGGCGTATCTC \\
\hline K-GFP & GACAGGGCCATCGCCAATTGG \\
\hline K-GFP & GGCGGTCGTTTCACCGTCAC \\
\hline
\end{tabular}




\subsubsection{Media}

\subsubsection{YPD-medium, $\mathrm{pH} 5.5$}

YPD is a rich medium for yeast cultures and contains:
$1 \%$ Bacto ${ }^{\circledR}$ Yeast Extract
$2 \%$ Bacto® Pepton
$2 \%$ D-glucose

\subsubsection{CM-medium, $\mathrm{pH} 5.6$}

$\mathrm{CM}$-medium is a synthetic medium for yeast cultures consisting of:
$0.67 \% \quad$ Yeast Nitrogen Base w/o amino acids
$2 \% \quad$ D-glucose
$0.0117 \% \quad$ L-alanine $0.0117 \%$ L-methionine*
$0.0117 \% \quad$ L-arginine $0.0117 \%$ L-phenylalanine
$0.0117 \% \quad$ L-asparagine $0.0117 \%$ L-proline
$0.0117 \% \quad$ L-aspartic acid $0.0117 \%$ L-serine
$0.0117 \% \quad$ L-cysteine $0.0117 \%$ L-threonine
$0.0117 \% \quad$ L-glutamine $0.0117 \%$ L-tyrosine
$0.0117 \% \quad$ L-glutamic acid $0.0117 \%$ L-valine
$0.0117 \% \quad$ L-glycine $0.0117 \%$ myo-inositol
$0.0117 \% \quad$ L-isoleucine $0.00117 \%$ p-aminobenzoic acid

Depending on selection conditions the following supplements were added:
$0.3 \mathrm{mM} \quad$ L-histidine
$0.4 \mathrm{mM} \quad$ L-tryptophan
$1.7 \mathrm{mM} \quad$ L-leucine
$0.3 \mathrm{mM} \quad$ adenine
$1 \mathrm{mM} \quad$ L-lysine
$0.2 \mathrm{mM} \quad$ uracil** $^{* *}$

Depending of the intended growth conditions of the cells different carbon sources were added:
$2 \%$
$2 \%$
D-glucose or
Lactate

* For overexpression experiments using a MET25 promoter L-methionine was excluded from the drop out mix.

** Uracil was resuspended in $0.5 \%$ sodium hydrogen carbonate solution. 


\subsubsection{SD-N-medium}

Nitrogen free SD (-N)-medium was used for nutrient starvation of yeast cells:

$0.67 \% \quad$ Yeast Nitrogen Base w/o amino acid and w/o ammonium sulfate

$2 \% \quad$ D-glucose

\subsubsection{1\% potassium acetate}

Potassium acetate is a medium used for sporulation (tetrade formation) or can also be used for carbon starvation:

$1 \%$ potassium acetate

\subsubsection{MV-medium, $\mathrm{pH} 5.5$}

For determination of the mating type of a haploid yeast strain, MV-medium was used:

$0.67 \%$ Yeast Nitrogen Base w/o amino acids

$2 \% \quad D$-glucose

\subsubsection{YPL-medium, $\mathrm{pH} 5.5$}

YPL is a rich medium for yeast cultures (similar to YPD) to enhance the synthesis of mitochondria and contains:

$1 \% \quad$ Bacto $\circledast$ Yeast Extract

$2 \%$ Bacto® Pepton

$2 \%$ lactate

\subsubsection{YPG-medium, $\mathrm{pH} 5.5$}
$1 \% \quad$ Bacto ${ }^{\circ}$ Yeast Extract
$2 \%$ Bacto $\otimes$ Pepton
$2 \%$ D-galactose

\subsubsection{LB-medium, $\mathrm{pH} 7.5$}

As standard growth medium for E. coli cultures LB-medium was used.
$1 \% \quad$ Bacto ${ }^{\circledR}$ Trypton
$0.5 \%$ Bacto $\circledast$ Yeast extract
$0.5 \%$ sodium chloride

For plasmid selection: $75 \mu \mathrm{g} / \mathrm{ml}$ ampicillin was added. For the use of BL21 (DE3) cells: $25 \mu \mathrm{g} / \mathrm{ml}$ was added. 


\subsubsection{SOC-medium, $\mathrm{pH} 7.5$}

For regeneration of electroporated E. coli cells SOC-medium was used

$\begin{array}{ll}2 \% & \text { Bacto® Trypton } \\ 0.5 \% & \text { Bacto® Yeast extract } \\ 0.4 \% & \text { D-glucose } \\ 10 \mathrm{mM} & \text { sodium chloride } \\ 10 \mathrm{mM} & \text { magnesium sulfate } \\ 10 \mathrm{mM} & \text { magnesium chloride } \\ 2.5 \mathrm{mM} & \text { potassium chloride }\end{array}$

3.1.5.10 Enriched sporulation medium

$1 \% \quad$ potassium acetate

$0.1 \% \quad$ Bacto ${ }^{8}$ Yeast extract

$0.05 \% \quad$ D-glucose

$0.01 \% \quad$ Amino-acids supplement powder mixture for sporulation*

$2 \% \quad$ Bacto agar

*Amino-acids supplement for sporulation medium:
$0.2 \%$
L-histidine
$1 \%$
L-leucine
$0.2 \%$
L-lysine
$0.2 \%$
L-uracil

\subsubsection{SD medium for SGA}

$0.17 \%$ yeast nitrogen base w/o amino acids or ammonium sulfate

$0.1 \% \quad$ MSG (L-glutamic acid sodiumsalt hydrate, Sigma),

$0.2 \% \quad$ amino-acids supplement powder mixture*,

$5 \% \quad 40 \%$ D-glucose

additional antibiotica/ amino acids for selection**

*Amino acids supplement powder mixture for synthetic media (complete)/ (DO - his/arg/lys)/ (DO - leu/arg/lys)
$0.3 \%$
L-adenine (Sigma)
$0.2 \%$
uracil (ICN)
$0.2 \%$
L-inositol
$0.02 \%$
para-aminobenzoic acid (Acros 5Organics)
$0.2 \%$
L-alanine
$0.2 \%$
L-arginine
$0.2 \%$
L-asparagine
$0.2 \%$
L-aspartic acid
$0.2 \%$
L-cysteine
$0.2 \%$
L-glutamic acid
$0.2 \%$
L-glutamine
$0.2 \%$
L-glycine 


$\begin{array}{ll}0.2 \% & \text { L-histidine } \\ 0.2 \% & \text { L-isoleucine } \\ 1 \% & \text { L-leucine } \\ 0.2 \% & \text { L-lysine } \\ 0.2 \% & \text { L-methionine } \\ 0.2 \% & \text { L-phenylalanine } \\ 0.2 \% & \text { L-proline } \\ 0.2 \% & \text { L-serine } \\ 0.2 \% & \text { Lthreonine } \\ 0.2 \% & \text { L-tryptophan } \\ 0.2 \% & \text { L-tyrosine } \\ 0.2 \% & \text { L-valine (Fisher) }\end{array}$

**

$100 \mu \mathrm{g} / 1 \quad$ canavanine

$100 \mu \mathrm{g} / 1 \quad$ thialysine

$200 \mu \mathrm{g} / 1 \quad$ G4 18

$100 \mu \mathrm{g} / 1 \quad$ clonNAT

\subsubsection{Antibodies}

Table 11: Anibodies used in this study TBST is containing $20 \mathrm{mM}$ Tris/ $\mathrm{HCl} \mathrm{pH} 7.6 ; 137 \mathrm{mM}$ sodium chloride; $0.1 \%$ Tween 20 (w/v)

\begin{tabular}{|c|c|c|}
\hline Name & Dilution & Source \\
\hline Mouse-Anti-GFP & $1: 2000$ in TBST $+1 \%$ milk & Roche, Mannheim \\
\hline Mouse-Anti-PGK & $1: 10000$ in TBST & $\begin{array}{c}\text { Molecular Probes, Leiden, } \\
\text { NL }\end{array}$ \\
\hline Rabbit-Anti-Ape1p & $1: 3000$ in TBST $+1 \%$ milk & Eurogentech, Belgium \\
\hline Rabbit-Anti-Aut7 & $1: 10000$ in TBST & M. Thumm \\
\hline Mouse-Anti-CPY & $1: 10000$ in TBST & $\begin{array}{c}\text { Molecular Probes, Leiden, } \\
\text { NL }\end{array}$ \\
\hline anti-mouse-HRPO-conjugate & $1: 10000$ in TBST $+1 \%$ milk & Dianova, Hamburg \\
\hline anti-rabbit-HRPO-conjugate & $1: 5000$ in TBST $+1 \%$ milk & Medac, Hamburg \\
\hline
\end{tabular}




\subsubsection{Kits}

Table 12: Kits used in this study

\begin{tabular}{ll}
\hline Name of product & Source \\
\hline Wizard Plus SV Minipreps DNA purification & Promega \\
System & \\
QIAquick Gel Extraction Kit & Quiagen, Hilden \\
QIAquick PCR Purification Kit & Quiagen, Hilden \\
QIAquick Nucleotide Removal Kit & Quiagen, Hilden \\
& \\
Rodeo ECL Western Blot Detection & USB \\
ECL PLUS Western Blotting Detection & Amersham, GE Healthcare
\end{tabular}

Reagents

\subsubsection{Chemicals}

Table 13: Chemicals used in this study

\section{Chemicals Source}

Acetic acid

Acetone

Adenine

Agarose

Ammoniumpersulfat

Ampicillin

Bacto Peptone

Bacto Tryptone

Bacto Yeast Extract

Bromphenolblue

BSA 100x

Canavanin

$\mathrm{CH}_{3} \mathrm{CO}_{2} \mathrm{~K}$ (acetic acid)

Chloramphenicole

Chloroforme

Chymostatin

clonNat (nourseotricine)

CompleteTM protease inhibitor

Cupric sulfate

D-Galactose

D-Glucose

DMSO

DNA-marker $(1 \mathrm{~kb})$

DNAse 1

dNTP

D-Sorbitol

DTT

ECL

$\mathrm{ECL}^{\mathrm{TM}}$ plus
Riedel-De Haën, Seelze

Roth, Karlsruhe

Roth, Karlsruhe

Roth, Karlsruhe

Roth, Karlsruhe

Roth, Karlsruhe

Becton Dickinson, Heidelberg

Becton Dickinson, Heidelberg

Becton Dickinson, Heidelberg

Riedel-De Haën, Seelze

NEB, Frankfurt

Sigma, Deisenhofen

Merck, Darmstadt

Roth, Karlsruhe

Roth, Karlsruhe

Merck, Darmstadt

Werner BioAgents, Jena

Roche, Mannheim

Sigma, Deisenhofen

Sigma, Deisenhofen

Roth, Karlsruhe

Sigma, Deisenhofen

NEB, Frankfurt

Invitrogen, Karlsruhe

NEB, Frankfurt

Roth, Karlsruhe

Roth, Karlsruhe

USB, Santa Clara, CA

GE Healthcare, München 


\begin{tabular}{|c|c|}
\hline Chemicals & Source \\
\hline EDTA & Roth, Karlsruhe \\
\hline Ethidium bromide & Roth, Karlsruhe \\
\hline $\mathrm{EtOH}$ & Roth, Karlsruhe \\
\hline Fidely Taq & USB, Santa Clara, CA \\
\hline FM4-64 & Invitrogen, Karlsruhe \\
\hline G418/Kanamycin & Roth, Karlsruhe \\
\hline Glusulase & PerkinElmer, Hamburg \\
\hline Glycerine & Roth, Karlsruhe \\
\hline Glycine & Roth, Karlsruhe \\
\hline Hepes/KOH & Roth, Karlsruhe \\
\hline Herring sperm DNA & Promega, Madison, USA \\
\hline HiDye & Sigma, Deisenhofen \\
\hline Hoechst 33342 & Molecular Probes, Freiburg \\
\hline IPTG & Roth, Karlsruhe \\
\hline Isopropanol & Roth, Karlsruhe \\
\hline $\mathrm{KCl}$ & Roth, Karlsruhe \\
\hline $\mathrm{KCl}$ & Merck, Darmstadt \\
\hline KH2PO4 & Roth, Karlsruhe \\
\hline Lactate & Sigma, Deisenhofen \\
\hline L-alanine & Sigma, Deisenhofen \\
\hline L-arginine & Sigma, Deisenhofen \\
\hline Lasparagine & Sigma, Deisenhofen \\
\hline L-aspartic acid & Sigma, Deisenhofen \\
\hline L-cysteine & Sigma, Deisenhofen \\
\hline Leupeptine & Sigma, Deisenhofen \\
\hline L-glutamic acid & Sigma, Deisenhofen \\
\hline L-glutamic acid sodiumsalt hydrate & Sigma, Deisenhofen \\
\hline L-glutamine & Sigma, Deisenhofen \\
\hline L-glycine & Sigma, Deisenhofen \\
\hline L-histidine & Sigma, Deisenhofen \\
\hline ligation buffer $1 \mathrm{x}$ & NEB, Frankfurt \\
\hline L-isoleucine & Sigma, Deisenhofen \\
\hline Lithium acetate & Sigma, Deisenhofen \\
\hline L-leucine & Sigma, Deisenhofen \\
\hline L-lysine & Sigma, Deisenhofen \\
\hline L-methionine & Sigma, Deisenhofen \\
\hline L-tryptophan & Roth, Karlsruhe \\
\hline Magnesium chloride & Roth, Karlsruhe \\
\hline Magnesium sulfate & Roth, Karlsruhe \\
\hline Methanol & Applichem, Darmstadt \\
\hline $\mathrm{Na}_{2} \mathrm{HPO}_{4}$ & Roth, Karlsruhe \\
\hline $\mathrm{NaCl}$ & Roth, Karlsruhe \\
\hline NA-desoxylat & Roth, Karlsruhe \\
\hline $\mathrm{NaOH}$ & Roth, Karlsruhe \\
\hline Paraquat & Sigma, Deisenhofen \\
\hline Pefablock & Sigma, Deisenhofen \\
\hline PEG 3350 & Sigma, Deisenhofen \\
\hline Phe TE & Roth, Karlsruhe \\
\hline Phe/Chl & Roth, Karlsruhe \\
\hline PMSF & Roth, Karlsruhe \\
\hline
\end{tabular}




\begin{tabular}{ll}
\hline Chemicals & \multicolumn{1}{c}{ Source } \\
\hline Poly-L-lysine & Sigma, Deisenhofen \\
DNA polymerase (Taq) & NEB, Frankfurt \\
DNA polymerase (Fideli Taq) & USB, Santa Clara, CA \\
Precision Plus Protein All Blue & Biorad, Munich \\
Standards & \\
Protease inhibitor cocktail & Sigma, Deisenhofen \\
Protogel (30 \%) & Biozym, Hessisch Oldendorf \\
Rapamycin & LCLabs, Woburn, USA \\
Restriction ezymes & NEB, Frankfurt \\
RNAseA & Applichem, Darmstadt \\
SDS & Sigma, Deisenhofen \\
Sepharose/slurry & GE Healthcare, München \\
Sequencing buffer & Applied Biosystems, Darmstadt \\
Skim milk powder & Granovita GmbH, Heimertingen \\
ß-ME & Roth, Karlsruhe \\
T4-Ligase & NEB, Frankfurt \\
TCA & Roth, Karlsruhe \\
Temed & Roth, Karlsruhe \\
Thialysin & Sigma, Deisenhofen \\
Tris & Roth, Karlsruhe \\
Triton X-100 & Roth, Karlsruhe \\
Tween 20 & Sigma, Deisenhofen \\
Uracil & Sigma, Deisenhofen \\
Vent Taq & NEB, Frankfurt \\
Yeast Nitrogen Base w/o amino & Becton Dickinson, Heidelberg \\
acids & \\
Yeast Nitrogen Base w/o amio acids & Becton Dickinson, Heidelberg \\
w/o ammonium sulfate & \\
\hline
\end{tabular}

\subsubsection{Consumables and equipment}

Table 14: Consumables and equipment used in this study

\section{Name of product}

Agarose gel equipment

Autoclave

Autoclave DX200

Bench

Blot shaker

Centrifuge

Centrifuge (refridgerating)

Centrifuge (small)

Centrifuge (small; refridgerating)

Chemical balance

Cuvettes

\section{Source}

Bio RAD Mini-SUB Cell GT

Adolf Wolf; Sanoclav

Systec

BDK Luf- u. Reinraumtechnik GmbH

GFL 3019

Eppendorf 5804

Eppendorf 5404R

Eppendorf 5415 D

Eppendorf 5415 R

Sartorius universal

Sarstedt cuvettes no. 67.742 


\begin{tabular}{|c|c|}
\hline Name of product & Source \\
\hline Cuvettes for electroporation & peq lab; $2 \mathrm{~mm}$ elektroporationsküvetten \\
\hline Electroporator & Eppendorf; Electroporator 2510 \\
\hline Freezer $\left(-20^{\circ} \mathrm{C}\right)$ & Liebherr \\
\hline Freezer $\left(-80^{\circ} \mathrm{C}\right)$ & Heareus; Hera freeze \\
\hline French pressure cell press & SIM AMINCO \\
\hline Gel dokumentation & UV solo; Transilluminator \\
\hline Incubator $\left(24 / 37^{\circ} \mathrm{C}\right)$ & Haraeus Kelvitron-t \\
\hline Incubator (small) & Eppendorf Thermomixer comfort \\
\hline Incubator shaking $\left(24 / 37^{\circ} \mathrm{C}\right)$ & New Brunswich Scientific; Innova 4200 \\
\hline LAS 3000 & Fujifilm \\
\hline Micromanipulator & ZEISS \\
\hline Microscope & ZEISS \\
\hline Microscope $\Delta$ vision, Olympus IX71 & Olympus; $\Delta$ vision Applied Precision, Inc \\
\hline Microscope slides (76x26 mm) & Menzel-Gläser \\
\hline Microscope cover slips & Menzel-Gläser \\
\hline Multivortex & IKA vibrax VXR basic \\
\hline PCR machine & Sensquest; Labcycler \\
\hline PCR machine & Eppendorf; Mastercycler gradient \\
\hline pH meter & Microprocessor $\mathrm{pH}$ Meter $\mathrm{pH} 537$ \\
\hline Photometer & Eppendorf Biophotometer \\
\hline Photometer & Amersham Ultrospec 100 pro \\
\hline Pinning tools & V \& P Scientific, Inc. (San Diego, CA) \\
\hline Pipettes & Eppendorf research \\
\hline Powersuplies & BioRAD; PowerPac HC \\
\hline PVDF membrane & Amersham Hybond-P; GE Healthcare \\
\hline Refrigerator $\left(4^{\circ} \mathrm{C}\right)$ & Bosch/ Liebherr \\
\hline Screen Roboter & Tecan; freedom evo \\
\hline OmniTrays & Nunc \\
\hline SDS-PAGE equipment & Bio RAD Mini Protean cell \\
\hline Sorvall centrifuge & Beckman router J2-MC \\
\hline special accuracy weighing machine & Sartorius handy \\
\hline Pipette tips, petri dishes, ... & Sarstedt/ Eppendorf \\
\hline Sterile filter & $\begin{array}{l}\text { Whatman; vacuflo, disposal vacuum } \\
\text { filtration system }\end{array}$ \\
\hline Transilluminator & Biometra TI 1 \\
\hline Vaccumpump & Diaphragm vacuum pump \\
\hline Vortex & Bender \& Hobein AG; Genie 2 \\
\hline Western Blot equipment & Bio RAD Trans Blot Cell \\
\hline Western Blot equipment (small) & Bio RAD Mini Trans Blot Cell \\
\hline Glassbeads & Schütt, Göttingen \\
\hline
\end{tabular}




\subsection{Cell biological methods}

\subsubsection{Cultivation of $S$. cerevisiae}

For preculture, yeast strains were inoculated with a sterile toothpick from an agar plate in liquid medium and grown over night $(12-14 \mathrm{~h})$ at $30^{\circ} \mathrm{C}$. For main-cultures the cells were diluted (depending on growth ability and requested $\mathrm{OD}_{600}$ ) in liquid media and grown over night $(12-14 \mathrm{~h})$ at $30{ }^{\circ} \mathrm{C}$. Special growth conditions needed to be adapted for some experiments, e.g. main-cultures for mitophagy experiments (see 3.4.5.3; up to $72 \mathrm{~h}$ in media containing $2 \%$ lactate).

\subsubsection{Storage of $S$. cerevisiae}

Yeast cells could be stored on agar plates at $4{ }^{\circ} \mathrm{C}$ up to one month. For long time storage yeast cells were cultured in liquid medium over night, transferred in $30 \%$ glycerol and frozen at $-80{ }^{\circ} \mathrm{C}$.

\subsubsection{Cultivation of E. coli}

For culturing of $E$. coli cells LB medium was used. The cells were inoculated in $5 \mathrm{ml} \mathrm{LB}$ and were incubated over night at $37^{\circ} \mathrm{C}$.

\subsubsection{Storage of E. coli}

E. coli cells could be stored for a few days in liquid culture or an LB agar plates at $4{ }^{\circ} \mathrm{C}$. For long time storage $500 \mu \mathrm{l}$ of $E$. coli liquid culture were transferred in $500 \mu 115 \%$ glycerol and frozen at $-80^{\circ} \mathrm{C}$.

\subsubsection{Mating of haploid yeast cells}

For mating of two different haploid strains (Mat a and Mat $\alpha$ ), $500 \mu 1$ of precultured cells were mixed by plating on agar plates or in liquid culture and incubated for at least 4 hours at $30{ }^{\circ} \mathrm{C}$. Developing zygotes were taken up by a micromanipulator and separated from haploid cells. 


\subsubsection{Mating type switch}

To switch the mating type, cells were transformed (see 3.3.3) with pHO plasmid. After two days of growth on selection medium one of the clones was grown over night in liquid YPG medium. The next day a 1:100 000 dilution of the culture was plated on YPD agarose. The grown colonies were tested for growth ability on CM -ura selection medium and colonies not able to grow could now be tested for mating type (see 3.2.7).

\subsubsection{Mating type determination}

To determine the mating type of a haploid cell, YR312 (Mat a) and YR320 (Mat $\alpha$ ) containing the Hisl allele were used as tester strains (Table 7). Both strains were applied on a MV-medium agar plate in two separate lines. The strain of interest was streaked through each of the tester strain lines and the plates were incubated for 2 days at $30{ }^{\circ} \mathrm{C}$. The strain of interest is only able to mate with the tester strain of contrary mating type, resulting in a diploid strain growing on MV-medium.

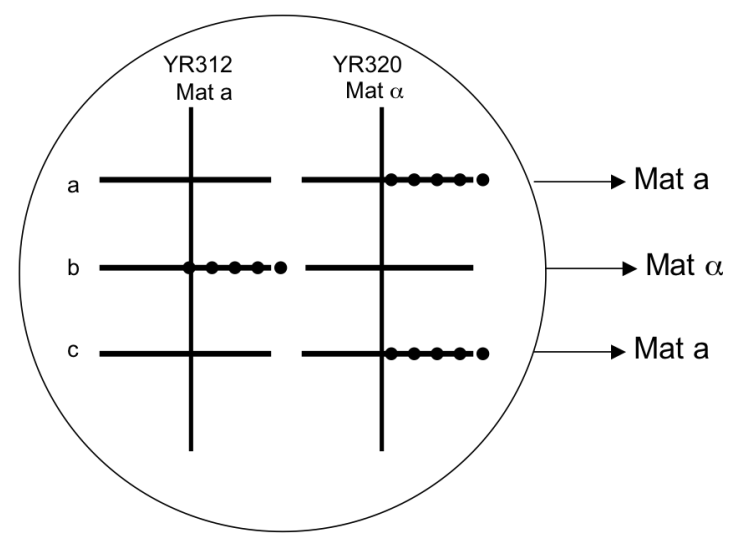

Figure 9: Scheme of mating type determination

\subsubsection{Sporulation of yeast cells}

For sporulation of diploid cells a preculture was inoculated and cultured over night at $30{ }^{\circ} \mathrm{C}$. On the next day the cells were transferred into enriched sporulation medium (see 3.1.5.10) or $1 \%$ potassium acetate medium and incubated for 5 days at $24{ }^{\circ} \mathrm{C}$. 


\subsubsection{Separation of haploid tetrads}

Sporulation of diploid yeast cells caused the formation of 4 haploid spores with a wrap (ascus) or so called tetrads. Therefore this method can be used to create haploid cells with different allele combinations out of a heterozygote diploid mother cell.

To separate haploid tetrads $200 \mu$ of a sporulated culture was washed with $\mathrm{H}_{2} \mathrm{O}$ by centrifugation and resuspended in $200 \mu 1 \mathrm{H}_{2} \mathrm{O} .4 \mu \mathrm{l}$ glusulase was added and incubated for 5 min at $30{ }^{\circ} \mathrm{C}$ while cautious shaking. Afterwards $800 \mu \mathrm{l}$ of $\mathrm{H}_{2} \mathrm{O}$ was added and incubated over night at $4{ }^{\circ} \mathrm{C}$. The next day, $6 \mu$ l of the cells were dropped on the top of an YPD plate and scattered carefully in one line to the bottom of the plate. After a few minutes of drying the tetrads could be taken up and divided by a gently knocking using a micromanipulator (ZEISS).

\subsubsection{Microscopy}

Light microscopy is a very powerful tool in science and probably the easiest and most rapid way to register macroautophagy by the direct visualisation of intravacuolar autophagic bodies (Prick and Thumm, 2008). For visualisation of yeast the cells either a light microscope (Zeiss Axioskope 2 or an inverted microscope (DeltaVision Spectris; Applied Precision)) were used. Both microscopes were equipped with a 100x objective.

\subsubsection{Test for the accumulation of autophagic bodies inside the vacuole}

The test for the accumulation of autophagic vesicles can be used for the detection of autophagic defects in deletion strains (Prick and Thumm, 2008).

Wild type cells treated with $1 \mathrm{mM}$ of the protease inhibitor PMSF are no longer able to breakdown the autophagic bodies in the vacuole. Using a 100x objective these vesicles can be observed as little wafting dots in the vacuole. In cells lacking compounds of the autophagic machinery as in the negative control $\operatorname{atg} 1 \Delta$, no autophagic bodies can be observed. In contrast, autophagic vesicles are visible in pep $4 \Delta$ cells lacking the breakdown machinery, even without the addition of PMSF.

\subsubsection{Fluorescence microscopy}

As a substantial part of this study, cells containing a fluorescence signal (e.g. GFP, RFP) were visualised and evaluated. The pictures were taken using a Zeiss Axioskope 2 with an 
AxioCam MRm and interpreted with by AxioVision software 4.5 (Zeiss) or the pictures were examined by an inverted fluorescence microscope (DeltaVision Spectris; Applied Precision) and analysed using soft-WoRx (Applied Precision, LLC). All fluorescence pictures were taken with corresponding filter sets: FITC, TRITC and DAPI.

For microscopy $5 \mu \mathrm{l}$ of yeast cells of interest were dropped on a glass slide and covered with a cover slip.

\subsection{Staining of the nucleus using Hoechst 33324}

The use of Hoechst 33324 is a very useful tool to stain nuclear DNA. Cells of interest were cultured to $\mathrm{OD}_{600} 0.5-6.10 \mu \mathrm{l} / \mathrm{ml}$ of $2 \mathrm{mM}$ Hoechst 33324 stock solution was added and the cells were further incubated for $15 \mathrm{~min}$ at $30{ }^{\circ} \mathrm{C}$ and used for microscopy.

\subsection{Staining of endosome and vacuole using FM4-64}

Cells of interest were cultured to $\mathrm{OD}_{600} 0.5-6$. After sedimentation at RT for $5 \mathrm{~min}$ at 2000 $\mathrm{rpm}$, the cells were resuspended in required medium of corresponding amount containing $2 \mu 1$ of FM4-64 stock solution ( $1 \mathrm{mg} / 100 \mu \mathrm{g}$ DMSO) and further incubated at $30^{\circ} \mathrm{C}$ for $30 \mathrm{~min}$. Afterwards they were transferred to fresh medium and used for microscopy (see 3.2.10.2) after a certain growth or starvation time (Prick and Thumm, 2008).

\subsubsection{Synthetic genetic array}

The Synthetic Genetic Array analysis (SGA) is a powerful high-throughput technique for exploring synthetic lethality. SGA allows the systematic construction of double mutants using a combination of mating and selection and was developed using the model organism $S$. cerevisiae. In SGA methodology a query gene deletion mutant is crossed to an entire genome deletion library consisting of 5000 viable deletion mutants available for yeast $S$. cerevisiae (Euroscarf; deletion mutant array (DMA); $\left.x_{(D M A)} \Delta:: K a n M X 4\right)$, to identify synthetic lethality, yielding to functional information of the query gene and the interacting genes. The result of such a screen highlights genes with similar function, which tend to interact with each other and genes with similar patterns of genetic interactions, which often encode products that tend to work in the same pathway or complex (Tong, 2001; Tong and Boone, 2006). 


\subsubsection{Generation of the query strain}

All strains of interest had to be generated. BY4741 $\operatorname{atg} 8 \Delta$, $\operatorname{atg} 15 \Delta$ and $\operatorname{atg} 21 \Delta$ were chosen for screening and therefore furthermore mentioned as query $\Delta$. Fore use in the screen, every query strain (deletion mutant) had to be mated with Y8205 (MAT $\alpha$ can1 $1:$ :STE2pr-his5 lyp $1 \Delta::$ STE3pr-LEU2 ura3 $\Delta 0$ leu2 $\Delta 0$ his $3 \Delta 1$ met15 $\Delta$; www.utoronto.ca/boonelab) to include several genetic attributes for selection during the screen and the zygotes were separated from haploid cells (see 3.2.5).

In the next step kanamycin resistance cassette (KanMX4) had to be switched to a nourseothricin resistance cassette (NatMX4). The method described in the literature (Tong and Boone, 2006) did not result in a successful switch, therefore the nourseothricin resistance cassette (NatMX4) was amplified by PCR using the plasmid pAG25 and the respective primers (Table 10). The resulting product was used for tranformation by homologue recombination (see 3.3.2). Afterwards the diploid strain containing NatMX4 was sporulated for 5 days in enriched sporulation medium (see 3.2.8).

The genetic modifications passed by Y8205 to the progeny allowed the subsequent selection for the haploid progeny of mating type a query $\Delta::$ NatMX4 can1 $1::$ STE2pr-his5

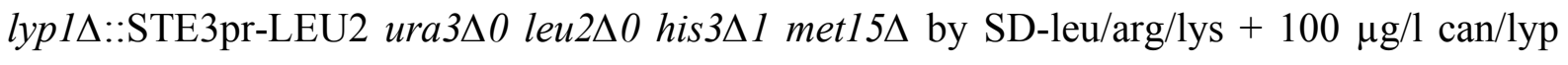
medium. As STE3pr-LEU2 reporter is only activated in MAT $\alpha$ cells, the use of medium lacking leucine allowed the selection for cells of mating type $\alpha$ and the further lack of arginine and lysine and the addition of $100 \mu \mathrm{g} / \mathrm{l}$ canavanin (can; a toxic analogue for arginine (Hoffmann, 1985)) and $100 \mu \mathrm{g} / 1$ thialysine (lyp; a toxic analogue to lysine (Sychrova and Chevallier, 1993) ensured the elimination of diploids (Mat a/ $\alpha$ ) and inhibited the growth of false positive Mat $\alpha / \alpha$ diploid mutations. This furthermore allowed to skip an additional step for separating the tetrads. 


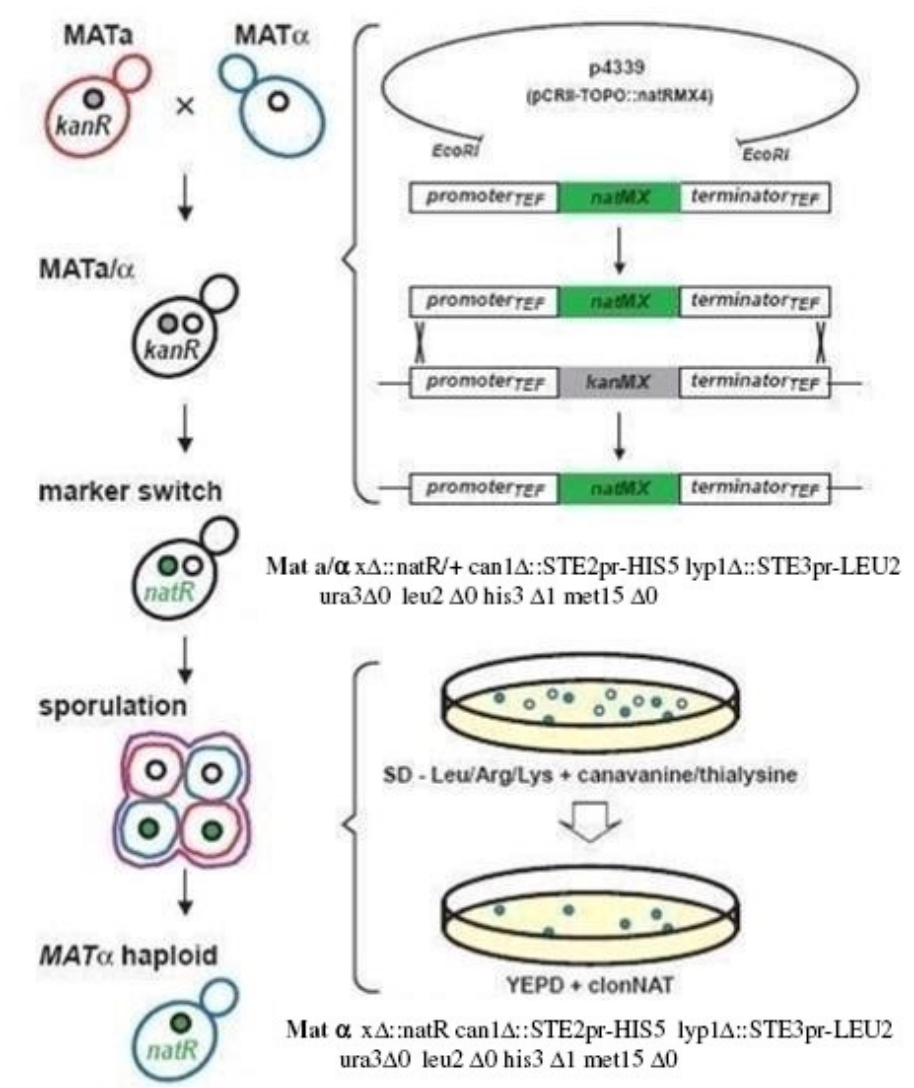

Figure 10: Preparation of the query strain

Deletion strain of interest (query strain) is prepared for the screen by mating with Y8250, switching of the resistance cassette (KanMX4 to NatMX4), sporulation and selection for the haploid progeny containing the deletion and the nourseothricin resistance ((Tong and Boone, 2006); modified).

\subsubsection{SGA - screen}

The screen, consisting of 5 pinning steps, was done with the whole deletion library available at Euroscarf, using a freedom evo screen roboter from Tecan in the laboratory of Prof. Braus (Department of Molecular Microbiology \& Genetics, University of Göttingen), or manual pinning tools (96 floating pin E-clip style manual replicator (VP408FH) and 384 floating pin E-clip style manual replicator (VP384F)) purchased from V \& P Scientific, Inc. (San Diego, CA). All pinning steps were done on OmniTrays (Nunc).

On the first day, the query strain (query $\Delta:: N a t M x 4)$ and DMA $\left(x_{(D M A)} \Delta:: K a n M X 4\right)$ were mated on YPD medium. After one day of growth, the colonies were transferred sporulation medium. After 5 days of sporulation the colonies were transferred to SD-his/arg/lys +100 $\mu \mathrm{g} / \mathrm{l}$ can/lyp medium to select for haploid progeny of mating type a, allowed by the resulting lowest level of inappropriate expression of HIS3 in Mat $\alpha$ and Mat a/ $\alpha$ cells. Also in this step the addition of $100 \mu \mathrm{g} / \mathrm{l} \mathrm{can} /$ and $100 \mu \mathrm{g} / 1$ lyp ensured the elimination of diploids (Mat a/ $\alpha$ ) and inhibited the growth of false positive Mat a/a diploid mutations. On the next day, the 
colonies were transferred to SD-his/arg/lys medium containing $200 \mu \mathrm{g} / \mathrm{l} \mathrm{G} 418$ and $100 \mu \mathrm{g} / \mathrm{l}$ can/lyp for selection $\left(x_{(D M A)} \Delta:: K a n M X 4\right)$. The last step included the switch to SD-his/arg/lys medium containing $100 \mu \mathrm{g} / \mathrm{l}$ clonNAT, $200 \mu \mathrm{g} / \mathrm{l} \mathrm{G418}$ and $100 \mu \mathrm{g} / \mathrm{l}$ can/lyp, resulting in the gowth of the haploid double mutant of mating type a (query $\Delta:: N a t M x 4 x_{(D M A)} \Delta:: \mathrm{KanMX} 4$

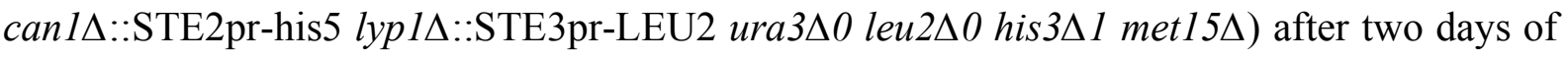
selection.

Two colonies per double mutant were pinned on the OmniTray plate and every plate was done in dublicates. This kind of double control resulted in about 53 plates containing 384 (192 x 2) colonies for each round.

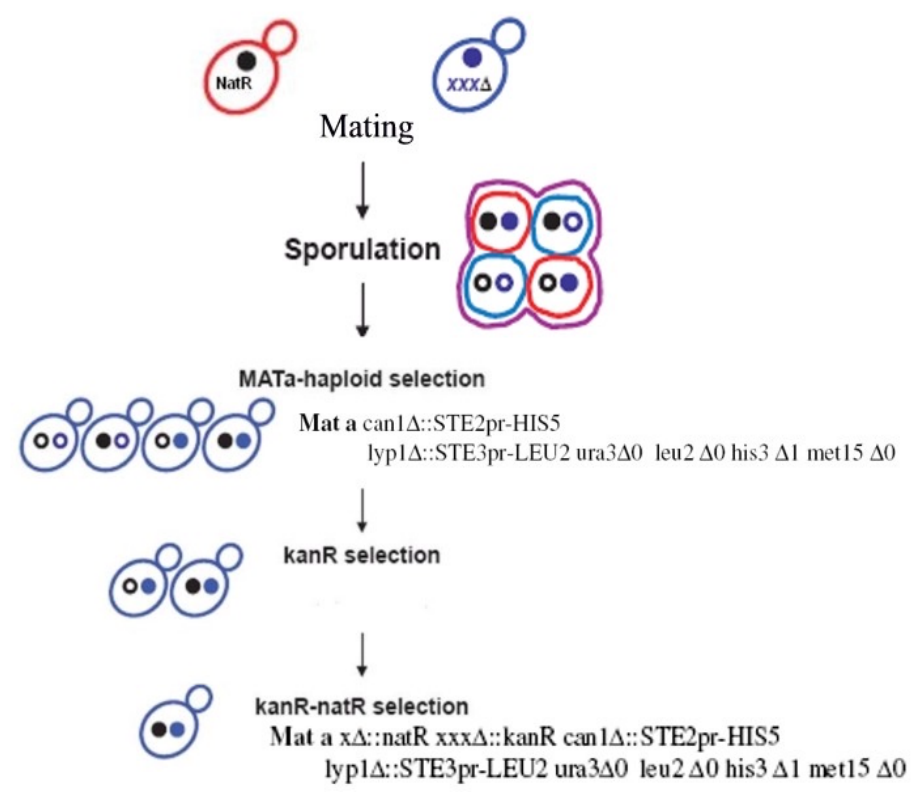

Figure 11: Synthetic genetic array methodology

Query strain containing nourseothricin resistance (query $\Delta:: N a t M X 4)$ and deletion strains containing kanamycin resistance $\left(x_{(D M A)} \Delta:: K a n M X 4\right)$ are mated, sporulated and the resulting haploid Mat a progeny are selected for double mutants ((Tong and Boone, 2006); modified) 


\subsection{Molecular biological methods}

\subsubsection{Isolation of chromosomal DNA}

For isolation of yeast chromosomal DNA, a $2 \mathrm{ml}$ over night culture was inoculated. The next day cells were spun down at $14000 \mathrm{rpm}$ for one minute and washed with $500 \mu 1 \mathrm{H}_{2} \mathrm{O}$. The pellet was resuspended in $200 \mu \mathrm{l}$ breaking buffer $(10 \mathrm{mM}$ Tris/ $\mathrm{HCl}, 100 \mathrm{mM} \mathrm{NaCl}, 1 \mathrm{mM}$ EDTA, $1 \%$ SDS and $2 \%$ TritonX-100) $200 \mu 1$ glass beads and $200 \mu 1$ Phe/Chl (50\% (v/v)

Phe TE, $50 \%(\mathrm{v} / \mathrm{v})$ chloroforme) were added and mixed for $20 \mathrm{~min}$ at $4{ }^{\circ} \mathrm{C}$. Afterwards 200 $\mu \mathrm{H}_{2} \mathrm{O}$ were added and the sample was centrifuged at $14000 \mathrm{rpm}$ for $10 \mathrm{~min}$ at room temperature. $300 \mu \mathrm{l}$ of the upper phase were transferred to a new $1.5 \mathrm{ml}$ reaction tube and the

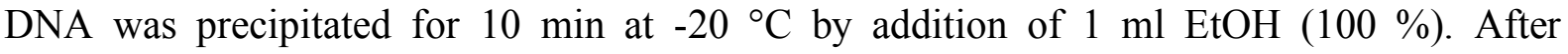
centrifugation at $14000 \mathrm{rpm}$ for $10 \mathrm{~min}$ at $4{ }^{\circ} \mathrm{C}$ the supernatant was discarded and the pellet resuspended in $400 \mu 1 \mathrm{H}_{2} \mathrm{O}+3 \mu \mathrm{l}$ RaseA $(10 \mathrm{mg} / \mathrm{ml})$. The RNA was digested for $5 \mathrm{~min}$ at $37{ }^{\circ} \mathrm{C}$ and the DNA again precipitated by adding $1 \mathrm{ml} \mathrm{EtOH}$ and $10 \mu 15 \mathrm{mM}$ ammonium acetate for $10 \mathrm{~min}$ at $-20^{\circ} \mathrm{C}$. After centrifugation at $14000 \mathrm{rpm}$ for $10 \mathrm{~min}$ at $4{ }^{\circ} \mathrm{C}$ the pellet was dried for a few minutes at $37{ }^{\circ} \mathrm{C}$ and resuspended in $30 \mu 1 \mathrm{H}_{2} \mathrm{O}$.

\subsubsection{Transformation of DNA}

A preculture of the cells of interest was prepared. On the next day 10 to $50 \mathrm{ml}$ main culture were inoculated with a dilution of 1:10. After an incubation of $2-6$ hours at $30^{\circ} \mathrm{C}$ the cells were harvested at an $\mathrm{OD}_{600} 0,5-0,8$ by centrifugation at $2000 \mathrm{rpm}$ for $5 \mathrm{~min}$, fallowed by two washing steps with water and one with LiOAc-Sorb $(100 \mathrm{mM}$ lithium acetate, $10 \mathrm{mM}$ Tris/acetate $\mathrm{pH}$ 8.0, $1 \mathrm{mM}$ EDTA, $1 \mathrm{M}$ D-Sorbitol). Furthermore the cells were resuspended in $100-500 \mu \mathrm{LiOAc}-S o r b$ and incubated at $30^{\circ} \mathrm{C}$ for $15 \mathrm{~min}$. Afterwards $50 \mu 1$ of the cells were added to $5 \mu \mathrm{l}$ herring sperm DNA (10 mg/ml), $300 \mu \mathrm{l}$ PEG in Li-TE (100 mM lithium acetate, $10 \mathrm{mM}$ Tris/acetate $\mathrm{pH}$ 8.0, $1 \mathrm{mM}$ EDTA, $40 \%$ (v/v) PEG 3350) and 3-10 $\mu 1$ of the DNA to be transformed. After an incubation step at $30{ }^{\circ} \mathrm{C}$ for $30 \mathrm{~min}$ the cells were heat shocked at $42{ }^{\circ} \mathrm{C}$ for $15 \mathrm{~min}$, sedimented at $2000 \mathrm{rpm}$ for $1 \mathrm{~min}$ and spread on selection medium. In case of DNA transformation for knock out by homologues recombination an additional incubation for 2 hours in YPD medium was necessary. 


\subsubsection{Transformation of plasmid-DNA}

For transformation of plasmid DNA ("Quick and dirty"), cells from an agar plate were resuspended in $300 \mu \mathrm{PEG}$ in Li-TE-buffer $(100 \mathrm{mM}$ lithium acetate; $10 \mathrm{mM}$ Tris/acetate $\mathrm{pH}$ 8.0; $1 \mathrm{mM}$ EDTA, $40 \%$ (v/v) PEG 3350). $5 \mu \mathrm{l}$ herring-sperm DNA (10 mg/ml) and $1-5 \mu \mathrm{l}$ plasmid DNA were added and the sample was incubated as described in chapter 3.3.2.

\subsubsection{Preparation of electrocompetent E. coli}

Plasmid tranformation in Escherichia coli is a standard procedure in the field of molecular biology (see 3.3.5), but can only be performed in competent cells.

For competent $E$. coli cells (Table 8), a main culture of $600 \mathrm{ml}$ LB-medium was inoculated $1: 100$ from an overnight culture and incubated at $37^{\circ} \mathrm{C}$ up to an $\mathrm{OD}_{600}$ of $0.6-0.7$, cooled down for $10 \mathrm{~min}$ on ice and spun down at $6500 \mathrm{rpm}$ for $8 \mathrm{~min}$ at $4{ }^{\circ} \mathrm{C}$. The cells were washed tree times with $0.51 \mathrm{H}_{2} \mathrm{O}$ and $20 \mathrm{ml}$ cold $10 \%$ (v/v) glycerol. After these washing steps the cells were resuspended in $2 \mathrm{ml}$ cold $10 \%(\mathrm{v} / \mathrm{v})$ glycerol and aliquoted (usually $40 \mu \mathrm{l}$ ) and stored at $-80{ }^{\circ} \mathrm{C}$.

\subsubsection{Plasmid transformation in E. coli}

In this study electroparation was used for transformation of plasmid DNA in E. coli cells. By an electric impulse the cell membrane is made permeable to DNA (Hanahan, 1991; Sheng, 1995).

Prepared electrocompetent E. coli (see 3.3.4) cells were thawn carefully on ice, $1-2 \mu 1$ of the DNA to be transformed was added. The reaction was transferred to a pre-chilled electroporation tube and electroporated at $2500 \mathrm{~V}$. Afterwards $900 \mu 1$ SOC-medium were added and the sample was incubated for 1 hour at $37^{\circ} \mathrm{C}$ at $220 \mathrm{rpm}$. After the incubation the cells were spun down, resupended in $100 \mu 1 \mathrm{H}_{2} \mathrm{O}$, spread on LB-plates containing $45 \mu \mathrm{g} / \mathrm{ml}$ ampicillin and incubated over night at $37^{\circ} \mathrm{C}$.

\subsubsection{Isolation of plasmid DNA from E. coli}

To isolate small amounts of clean DNA the Wizard Plus SV Kit from Promega was used as described in the manufacturer's manual. 


\subsubsection{Restriction of DNA}

DNA was digested with specific restriction enzymes to analyse DNA or prepare DNA for ligation (see 3.3.10). 1 - $5 \mu$ l DNA, $1.5 \mu$ l buffer (10 x concentrated), $1.5 \mu 1$ BSA (10 x concentrated) if needed and 2 - $10 \mathrm{U}$ enzyme were used $\left(\sum=15 \mu 1\right)$. The reaction was incubated for 1 - 2 hours at optimal enzyme temperature (normally $37^{\circ} \mathrm{C}$ ). All reactions were done following the manufacturers advice from NEB. The reaction was mixed with $1.5 \mu 1$ of DNA sample buffer (1 M Tris/HCl H 8.0; $50 \%$ (v/v) glycerol; $0.1 \%$ (w/v) bromphenolblue) and tested by using DNA agarose gel electrophoreses. For preparative digestions all standard reactions were scaled up and used for DNA gel extraction (see 3.3.9).

\subsubsection{DNA agarose gel electrophoresis}

The DNA agarose gel electrophoresis is a standard method to separate DNA fragments by size using their behaviour in an electric field (Brody and Kern, 2004). Dependent on the size of these fragments agarose gels with an concentration of 0.8 to $1.5 \%$ (w/v) agarose with $1 \mu \mathrm{g}$ / $\mathrm{ml}$ ethidiumbromide (to allow the detection of the DNA under UV-light $(254 \mathrm{~nm})$ ) in TAE buffer (40 mM Tris/acetate $\mathrm{pH} 8.1 ; 2$ mM EDTA; $0.114 \%$ acetic acid) were prepared in an electrophoresis chamber containing TAE buffer. Furthermore the DNA samples were prepared with DNA sample buffer (1 M Tris/HCl pH 8.0; $50 \%$ (v/v) glycerol; $0.1 \%$ (w/v) bromphenolblue), loaded on the agarose gel and separated for 20 - 30 min by running up to a voltage of $120 \mathrm{~V}$. For determination of DNA fragment size a standard ladder was loaded (TriDye $1 \mathrm{~kb}, \mathrm{NEB}$ ) (Table 15).

Table 15: Standard Tri Dye $1 \mathrm{~kb}$

$\begin{array}{ccccccccccc}\text { Fragment } & 1 & 2 & 3 & 4 & 5 & 6 & 7 & 8 & 9 & 10 \\ \text { Size (kb) } & 10 & 8 & 6 & 5 & 4 & 3 & 2 & 1.5 & 1 & 0.5\end{array}$

\subsubsection{DNA gel extraction}

To purify DNA fragments from agarose gels the QIAEX II Gel Extraction Kit from Qiagen was used as described in the manufacturer's manual. 


\subsubsection{Ligation of DNA fragments}

For ligation of DNA fragments into a vector, 2 - $6 \mu$ insert DNA (prepared by restriction; see 3.3.7), 0.5 - $3 \mu$ l vector DNA (prepared with corresponding restriction enzymes), $2.5 \mathrm{U}$ T4DNA-ligase and $1 \mu 1$ ligation buffer $(10 \mathrm{x}$ concentrated $)$ were filled up to $10 \mu 1$ with $\mathrm{H}_{2} \mathrm{O}$ and incubated overnight at $16{ }^{\circ} \mathrm{C}$. The next day $1-2 \mu \mathrm{l}$ of the sample could be transformed into E. coli (see 3.3.5).

\subsubsection{Polymerase chain reaction (PCR)}

The polymerase chain reaction (PCR) is a method to amplify a DNA sequence by using a DNA polymerase (Saiki, 1988). For $100 \mu \mathrm{l}$ PCR reaction, $10 \mu \mathrm{l}$ PCR buffer $(10 \mathrm{x}$ concentrated), $100 \mathrm{pmol}$ per oligonucleotide (forward and reverse; Operon), $1 \mu \mathrm{l}$ template DNA, $1 \mu \mathrm{d}$ dNTP mix (25 mM each dNTP), 1 - $2 \mu \mathrm{l}$ DMSO and up to $5 \mathrm{U}$ Taq DNA polymerase were mixed. Small fragments were amplified using the Taq DNA polymerase (NEB) and longer DNA fragments by using the proof reading polymerase Fideli Taq (USB). After a first step of DNA denaturation, 30 cycles of denaturation, annealing and elongation followed, completed by a last step of elongation. The exact program, concerning temperature and duration of cycles, was specially designed for each experiment, depending on the properties of the oligonucleotides and product size. The synthesised product could be evaluated by using the method of agarose gel electrophoresis (see 3.3.8).

\subsubsection{Sequencing of DNA}

For control, new cloned constructs were prepared for sequencing. $1 \mu \mathrm{l}$ of template DNA, $1 \mu 1$ sequencing mix (polymerase; dNTP mix with fluorescent dyes; $30 \mathrm{mM}$ magnesium chloride and buffer substances), $1 \mu$ l oligonucleotide ( 1 : 100; compared to usual PCR), $1.5 \mu 1$ sequencing buffer and $6 \mu 1 \mathrm{H}_{2} \mathrm{O}$ were used for PCR reaction. The PCR program included a 10 s denaturation step at $95{ }^{\circ} \mathrm{C}, 25$ cycles of annealing for $5 \mathrm{~s}$ at $50{ }^{\circ} \mathrm{C}$ and elongation for $4 \mathrm{~min}$ at $60{ }^{\circ} \mathrm{C}$. The PCR product was precipitated by addition of $1 \mu 13 \mathrm{M}$ sodium acetate $(\mathrm{pH}$ 5.2), $1 \mu 1125 \mathrm{mM}$ EDTA and $50 \mu \mathrm{l}$ ethanol (100\%, RT). After centrifugation at $13000 \mathrm{rpm}$ for $10 \mathrm{~min}$ at RT, the sediment was washed with $70 \%$ ethanol and dried for a few minutes. 15 $\mu 1$ HiDye (formamide) were added to stabilise the DNA. The reactions were analysed by the department of developmental biology of the Georg-August-Universität Göttingen. 


\subsubsection{Construction of knock out strains}

For construction of knock out strains, the method of homologous recombination was used. Homologous recombination is a type of genetic recombination in which nucleotide sequences are exchanged between two similar or identical molecules of DNA. Using well-directed primers for construction of the new DNA- cassette, specific DNA sequences can be specifically exchanged (Figure 12).

The knockout construct, synthesised by a PCR reaction using corresponding primers (Table 10) (Longtine, 1998; Janke, 2004), was adopted for competent yeast transformation (see 3.3.2).

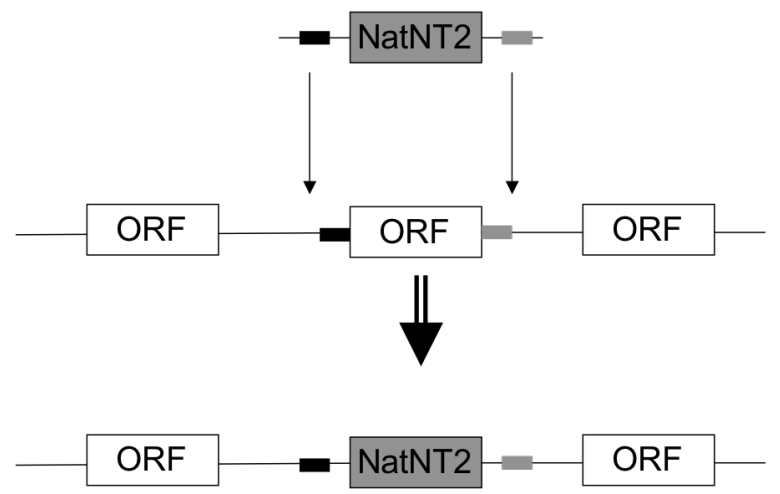

Figure 12: Scheme for construction of a knockout strain

((Tong and Boone, 2006); modified)

\subsubsection{Chromosomal C-terminal fusion with a GFP-taq}

A common approach is furthermore the chromosomal fusion of the gene of interest with a GFP taq. Most GFP fusion strains are available at Invitrogen (www.invitrogen.com), others were generated in this thesis. For example the chromosomal GFP-fusion of Om45 (Kanki, 2009a). Similar to knock out strain construction, the method of homologue recombination was used, including the PCR reaction using corresponding primers (Table 10) and the competent yeast transformation (see 3.3.2) (Longtine, 1998). 


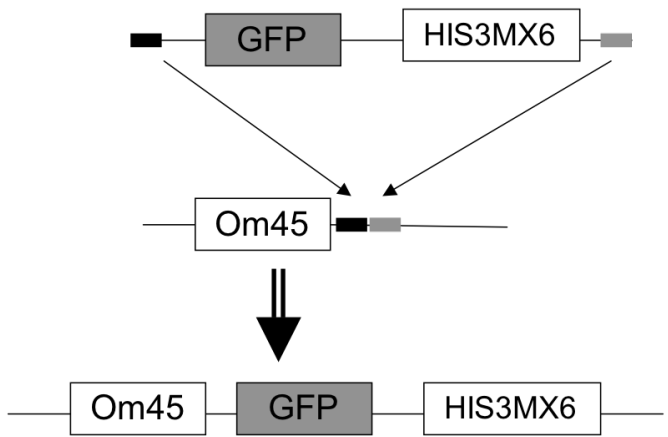

Figure 13: Scheme for the construction of chromosomal Om45-GFP ((Huh, 2003); modified)

\subsubsection{Plasmid construction}

several plasmids were constructed in this study

\section{Om45-GFP/Idh1-GFP}

The plasmids pOm45-GFP and pIdh1-GFP were constructed by homologues recombination for use in mitophagy experiments. Therefore the vector pRS316 and specific primers, containing the purposed intersection for pRS316 (Table 10), were used. These primers were used for a PCR (see 3.3.11) reaction to amplify Om45-GFP, which had to be integrated into pRS316. As template $5 \mu \mathrm{l}$ of chromosomal DNA isolated from Om45-GFP/Idh1-GFP (Invitrogen) were used. The resulting DNA fragment was purified by gel electrophoresis (3.3.8). Furthermore $6.5 \mu \mathrm{l}$ of the resulting DNA fragment and $3.5 \mu \mathrm{l}$ of Sma I digested pRS316 were used for competent yeast tranformation into matching knock out strains $(o m 45 \Delta / i d h 1 \Delta)$.

The grown yeast colonies were used for plasmid rescues: $1.5 \mathrm{ml}$ of an over night preculture were centrifuged at $14000 \mathrm{rpm}$ for $1 \mathrm{~min}$ at room temperature. After washing with $500 \mu 1$ $\mathrm{H}_{2} \mathrm{O}$, the cells were resuspended in $200 \mu \mathrm{l}$ breaking buffer $(100 \mathrm{mM} \mathrm{NaCl}, 10 \mathrm{mM}$ Tris/ $\mathrm{HCl}$, $1 \mathrm{mM}$ EDTA, $1 \%$ (w/v) SDS, $2 \%$ (v/v) Triton-X-100; ph 8.0). $200 \mu 1$ glass beads and $200 \mu 1$ Phenol/Chloroform (50\% (v/v) Phenol, $50 \%$ (v/v) Chloroform) were added and the reaction was mixed at $4{ }^{\circ} \mathrm{C}$ for $5 \mathrm{~min}$. The cells were centrifuged at $14000 \mathrm{rpm}$ for $10 \mathrm{~min}$ and $50 \mu \mathrm{l}$ of the upper phase were transferred into a new reaction tube. The DNA was then precipitated at $20{ }^{\circ} \mathrm{C}$ for $20 \mathrm{~min}$ by adding $140 \mu \mathrm{l}$ ethanol $(100 \%$, ice cold) and $5 \mu \mathrm{l} 3 \mathrm{M}$ sodiumacetate/acetic acid ( $\mathrm{pH}$ 5.2). After an additional centrifugation step for $10 \mathrm{~min}$ at $14000 \mathrm{rpm}$, the supernatant was removed and the sediment was washed with $70 \%$ ethanol (cold) and dried at room temperature. Afterwards the plasmid DNA was resuspended in $30 \mu 1$ 
$\mathrm{H}_{2} \mathrm{O}$ and could be stored at $-20{ }^{\circ} \mathrm{C}$ or used for transformation in electrocompetent $E$. coli cells and subsequently for sequencing (see 3.3.12).

\section{su9mtDHFR-GFP (mito-GFP)}

Another plasmid, which was constructed in this study is su9-mt-DHFR-GFP, to detect mitophagy, furthermore referred to mito-GFP.

The use of well-directed primers (Table 10) in PCR to amplify a Su9-DHFR sequence (AG Rehling; Biochemistry II; Göttingen), containing specific restriction sites at the beginning and the end, allowed the insertion of this fragment (digested with BamH I and Hind III) into the vector pUG35 by ligation. Resulting in a plasmid encoding the Su9-DHFR sequence followed by a GFP-tag under control of a MET promoter.

\section{GST-Shp1}

For pull down experiments (see 3.4.7) recombinant GST-fusions of Shp1 were needed.

First step for construction of the plasmids encoding the recombinant GST-Shp1 was a polymerase chain reaction (see 3.3.11) using chromosomal wild type DNA as template and according primers (Table 10), containing restriction sites for Eco R I and Xho I and a vector gene overlapping sequence. The resulting PCR products were treated with the restriction enzymes Eco R I and Xho I (see 3.3.7) and ligated in frame into the EcoR I and Xho I restricted vector pGEX-4T-3 (Amersham) (see 3.3.10). The ligation product was transformed into electrocompetent $E$. coli cells (DH5 $\alpha$ ) (see 3.3.5) and tested for mutations by sequencing (see 3.3.12). Positive plasmids without mutations were transformed in E. coli BL21 (DE3) RIPL cells (Table 8) and used for pull down experiments (see 3.4.7). 


\subsection{Protein biochemical methods}

\subsubsection{Alkaline lysis}

For protein lysates via alkaline lysis as described in literature (Horvath and Riezman, 1994), $2 \mathrm{OD}_{600}$ of cells were used. The cells were centrifuged and resuspended in $1 \mathrm{ml}$ of cold $\mathrm{H}_{2} \mathrm{O}$, $150 \mu \mathrm{l}$ of lysis solution ( $925 \mu \mathrm{l}$ of $2 \mathrm{M} \mathrm{NaOH}(4 \mathrm{~g}$ in $50 \mathrm{ml})$ and $75 \mu 1 \mathrm{~B}-\mathrm{ME}$ ) were added and the reaction was incubated on ice for $10 \mathrm{~min}$ by vortexing every 2 minutes. To stop the reaction, $150 \mu 150 \%$ TCA were added and the lysed cells could be stored at $-20{ }^{\circ} \mathrm{C}$ over night or incubated on ice for $10 \mathrm{~min}$. Afterwards the tubes were centrifuged at $10000 \mathrm{~g}$ for 10 min at $4{ }^{\circ} \mathrm{C}$, supernatants were removed and the pellets were washed with acetone and dissolved in $100 \mu \mathrm{l}$ 1x Laemmli buffer (6 x Laemmli buffer $\mathrm{pH}$ 6.8: $0.35 \mathrm{M}$ Tris/ $\mathrm{HCl}, 10.28$ $\%$ SDS, $36 \%$ Glycerol, $5 \%$ ß-ME (add freshly), $0.012 \%$ bromphenoleblue) by conscientious shaking at $30{ }^{\circ} \mathrm{C}$ for $20 \mathrm{~min}$. After centrifugation at $10000 \mathrm{~g}$ for $5 \mathrm{~min}$, the samples were used for SDS-PAGE (see 3.4.3) or could be stored at $-20{ }^{\circ} \mathrm{C}$.

\subsubsection{Cell lysis for the detection of Atg8-PE}

The lipidation of Atg8, via an ubiquitin like conjugation system (see 2.2.1.2) and the delipidation via Atg4, are necessary for autophagosome formation (Kirisako, 2000). Strains defective in autophagy therefore were tested for impairment in the lipidation of Atg8.

To detect the connection of Atg8 to phosphatidylethanolamine (PE), cells have to be disrupted in a very smooth manner, using a modified method for cell lysis (Suzuki, 2001).

$60 \mathrm{OD}_{600}$ were harvested, centrifuged at $3000 \mathrm{rpm}$ for $3 \mathrm{~min}$ at RT and the supernatant was discarded. The cell pellet could be stored on ice (or $-20^{\circ} \mathrm{C}$ ) or directly resuspended in $500 \mu \mathrm{l}$ lysismix $(500 \mu \mathrm{l}$ lysis buffer $(50 \mathrm{mM}$ Hepes/KOH pH 7.5, $140 \mathrm{mM} \mathrm{NaCl}, 1 \mathrm{mM}$ EDTA, $10 \%$ glycerin, $0.05 \%$ Na-desoxylat, $2 \%$ Triton X-100, $0.1 \%$ SDS) containing furthermore $10 \mu 1100 \mathrm{mM}$ PMSF, $20 \mu 150$ x complete ( 1 tab in $1 \mathrm{ml}$ lyse buffer) and $1 \mu 1$ 1000x inhibitormix ( $1 \mu \mathrm{g} / \mathrm{ml}$ Pepstatin; $1 \mu \mathrm{g} / \mathrm{ml}$ leupeptin; $20 \mu \mathrm{g} / \mathrm{ml}$ chymostatin; $20 \mu \mathrm{g} / \mathrm{ml}$ antipain; $1 \mathrm{mM}$ benzamidin; $10 \mu \mathrm{g} / \mathrm{ml}$ aprotinin in DMSO)). Afterwards $200 \mu 1$ glass beads were added and the sample was vortexed for $10 \mathrm{~min}$ at $4{ }^{\circ} \mathrm{C}$, centrifuged $5 \mathrm{~min}$ at $3000 \mathrm{rpm}$ and $200 \mu \mathrm{l}$ of the supernatant were transferred into a new reaction tube. After the addition of $40 \mu 1$ 6x laemmli buffer (pH 6.8: 0.35 M Tris/HCl; $10.28 \%$ SDS; $36 \%$ glycerol, $5 \%$ ß-ME 
(added freshly); $0.012 \%$ bromphenoleblue) the samples were used for SDS-PAGE (see 3.4.3) or could be stored at $-20{ }^{\circ} \mathrm{C}$.

\subsubsection{SDS-PAGE}

The so called SDS-PAGE (sodium dodecyl sulfate polyacrylamide electrophoresis) is a technique to separate proteins according to their electrophoretic mobility (Laemmli, 1970). First a separating gel (Table 16) was prepared. For polymerisation APS and Temed were added. After filling the gel into the designated equipment, it was covered with a thin layer of isopropanol. After the polymerisation and the further displacement of the isopropanol, the stacking gel could be coated on the separating gel (Table 16). Subsequent to the polymerisation of the stacking gel, $10-15 \mu l$ of the prepared samples and, for further determination of the size of the proteins, a standard ladder (Precision Plus Protein All Blue Standard, BioRad) (Table 17) were loaded. The gel was run up to a voltage of $150 \mathrm{~V}$.

Table 16: Contents of SDS-PAGE

\begin{tabular}{lllllll} 
Separating gel & $2 \times 7 \%$ & $2 \times 8 \%$ & $4 \times 8 \%$ & $2 \times 10 \%$ & $2 \times 12 \%$ & $2 \times 15 \%$ \\
\hline & & & & & & \\
$\mathrm{H}_{2} \mathrm{O}$ & $5,1 \mathrm{ml}$ & 4,7 & 9,4 & 3,9 & 3,4 & 2,4 \\
$1,5 \mathrm{M}$ Tris & $2,5 \mathrm{ml}$ & 2,5 & 5 & 2,5 & 2,5 & 2,5 \\
$(\mathrm{pH} 8.8)$ & & & & & & \\
Protogel & $2,3 \mathrm{ml}$ & 2,7 & 5,4 & 3,5 & 4 & 5 \\
SDS & $100 \mu \mathrm{l}$ & 100 & 200 & 100 & 100 & 100 \\
APS & $100 \mu \mathrm{l}$ & 100 & 200 & 100 & 100 & 100 \\
TEMED & $5 \mu \mathrm{l}$ & 5 & 10 & 5 & 5 & 5
\end{tabular}

Stacking gel 2

$\begin{array}{ll}\mathrm{H}_{2} \mathrm{O} & 6,1 \mathrm{ml} \\ 0,5 \mathrm{M} \text { Tris }(\mathrm{pH} 6.8) & 2,5 \mathrm{ml} \\ \text { Protogel } & 1,3 \mathrm{ml} \\ \text { SDS } & 100 \mu \mathrm{l} \\ \text { APS } & 100 \mu \mathrm{l} \\ \text { TEMED } & 10 \mu 1\end{array}$

Table 17: Precision Plus Protein All Blue Standard

$\begin{array}{lccccccccc}\text { Line } & 1 & 2 & 3 & 4 & 5 & 6 & 7 & 8 & 9 \\ \text { Size (kDA) } & 250 & 150 & 100 & 75 & 50 & 37.5 & 25 & 15 & 10\end{array}$




\subsubsection{Western Blot}

To blot the proteins separated by SDS-PAGE on PVDF-membranes based on the established standard method of western blot (Renart, 1979; Towbin, 1979). The wet blot system from BioRad was used. The SDS-gel, the PVDF-membrane and whatmanpaper were inserted into the blot chamber from BioRad (Figure 14) and filled up with wet blot buffer (192 mM glycin, $25 \mathrm{mM}$ Tris and $20 \%$ methanol). The transfer time was chosen to be at least 4 hours or over night at $4{ }^{\circ} \mathrm{C}$ with $70 \mathrm{~mA}$ per $45 \mathrm{~cm}^{2}$ membrane size. Afterwards the PVDF-membranes were incubated in TBST buffer $(20 \mathrm{mM}$ Tris/ $\mathrm{HCl} \mathrm{pH} 7.6 ; 137 \mathrm{mM}$ sodium chloride; $0.1 \%$ Tween $20(\mathrm{w} / \mathrm{v}))$ containing $10 \%(\mathrm{w} / \mathrm{v})$ skim milk powder) for $30 \mathrm{~min}-1 \mathrm{~h}$ at RT or overnight at 4 ${ }^{\circ} \mathrm{C}$ to block all unspecific binding sites on the membrane. The blots were washed 3 times with 25 - $50 \mathrm{ml}$ TBST for $5 \mathrm{~min}$. Furthermore the membranes were incubated in TBST buffer containing the primary antibody (Table 11) for at least two hours at RT or overnight at $4{ }^{\circ} \mathrm{C}$. The immunoblots were washed 6 times with 25 - $50 \mathrm{ml}$ TSBT buffer for $5 \mathrm{~min}$ and then incubated in TBST buffer containing $1 \%$ skim milk powder (w/v) and the secondary antibody (Table 11) for $45 \mathrm{~min}$. After six times of $5 \mathrm{~min}$ washing steps with TBST, the blots were developed with ECL (USB) or ECL ${ }^{\mathrm{TM}}$ Plus (Amersham) following the manufacturer's advice. Afterwards the immunoblots were analysed using a LAS-3000 (Fujifilm). Quantitative statistics from blots were done using the AIDA Software, Version 4.06.116.

For the detection using another antibody, the immunoblots were stripped by shaking in $10 \%$ acetic acid for 10 min followed by three washing steps with TBST for $10 \mathrm{~min}$. Afterwards the immunoblots were incubated for $30 \mathrm{~min}-1 \mathrm{~h}$ in TBST containing $1 \%$ slim milk powder, washed and incubated with the primary antibody of interest (see above).
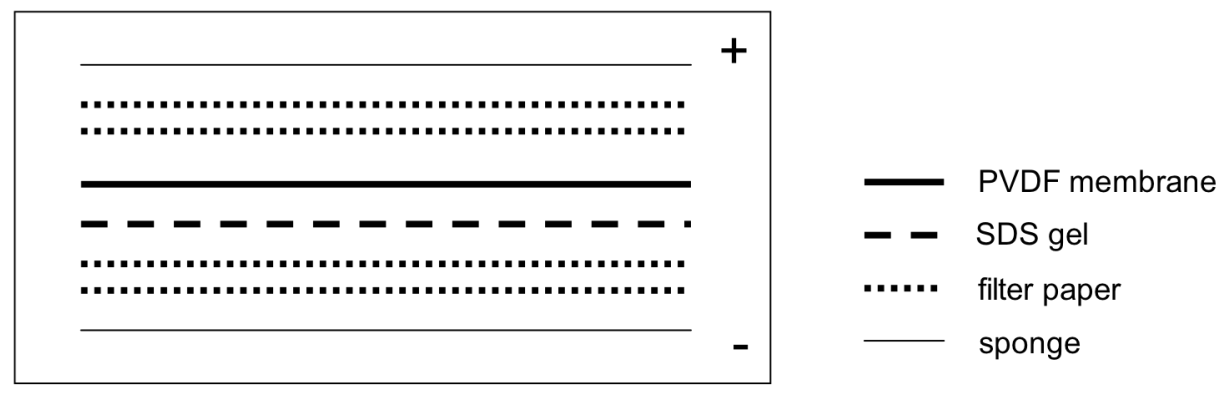

Figure 14: Scheme of western blot equipment 


\subsubsection{Measurement of autophagy dependent degradation of GFP-fused proteins}

\subsubsection{Macroautophagy}

For measurement of autophagy dependent degradation of GFP-fused proteins the strains of interest were transformed with GFP-Atg8 $(\sim 39 \mathrm{kDa})$ or Pgk1-GFP $(\sim 71 \mathrm{kDa})$ and treated as described in literature (Meiling-Wesse, 2002; Klionsky, 2007; Welter, 2010). Upon dlivery of GFP-Atg8 or Pgk1-GFP into the vacuole, the proteins are rapidly degraded, whereas the released free GFP remains relatively stable. Accordingly, the appearance of free GFP can be used to monitor autophagy (Klionsky, 2007).

Cells were grown in a main culture to stationary phase. $20 \mathrm{OD}_{600}$ were washed with SD-N medium, resuspended in $2 \mathrm{ml}$ of SD-N and incubated at $30{ }^{\circ} \mathrm{C} .200 \mu 1\left(2 \mathrm{OD}_{600}\right)$ cells were taken at defined time points $(0,2,4$ and $6 \mathrm{~h}$ ) and alkaline lysed (see 3.4.1).

\subsubsection{PMN}

For measurement of the selective piecemeal microautophagic degradation of the nucleus, the strains of interest were transformed with GFP-Osh1 ( 180 kDa) and treated as described (see 3.4.5.1) (Krick, 2008b).

\subsubsection{Mitophagy}

Two methods for detection of mitophagy by western blot were used. In the strains of interest GFP was chromosomally integrated after the Om45 locus (Om45-GFP::HIS3X6; see 3.3.14) $(\sim 71 \mathrm{kDa})$ (Kanki, 2009a) or they were transformed with the plasmid mito-GFP ( $\sim 48 \mathrm{kDa})$ (constructed in this thesis, see 3.3.15). Cells were grown for preculture in selection medium containing $2 \%$ glucose over night and transferred to main-culture medium containing $2 \%$ lactate* $\left(0.5 \mathrm{OD}_{600} / \mathrm{ml}\right)$. The next day $0.05 \mathrm{mM} \mathrm{H}_{2} \mathrm{O}_{2}, 2 \mathrm{mM}$ paraquat or $0.2 \mu \mathrm{g} / \mathrm{ml}$ rapamycin were added to $20 \mathrm{OD}_{600}$ cells and incubated at $30{ }^{\circ} \mathrm{C} .2 \mathrm{OD}_{600}$ cells were taken at defined time points $(0,2,4,6 \mathrm{~h})$ and alkaline lysed (see 3.4.1) or the cells were grown up to $72 \mathrm{~h}$ in main culture and $2 \mathrm{OD}_{600}$ cells were taken at defined time points $(24,48$ and $72 \mathrm{~h})$ and alkaline lysed.

*For cells transformed with the plasmid mito-GFP, main culture medium furthermore lacking methionine (-Met) was used. 


\subsubsection{Pull down of GST-Shp1}

((Wu, 2006); (Smith and Johnson, 1988); modified)

\subsubsection{Purification of recombinant GST-Shp1}

For preculture, $10 \mathrm{ml} \mathrm{LB}$ containing $75 \mu \mathrm{g} / \mathrm{ml}$ ampicillin and $25 \mu \mathrm{g} / \mathrm{ml}$ chloramphenicol were inoculated with $E$. coli cells containing the GST-Shp1 construct. The next day, the cultures were diluted $1: 10$ and incubated at $30{ }^{\circ} \mathrm{C}$ for 2 hours. Afterwards the expression of recombinant protein was induced by adding $0.2 \mathrm{mM}$ IPTG and an incubation for additional 4 hours at $30^{\circ} \mathrm{C}$.

The cells were harvested by centrifugation at $5000 \mathrm{rpm}$ for $10 \mathrm{~min}$ and solubilised using a french press (SIM AMINCO). Therefore the sediments were resuspended in $5 \mathrm{ml}$ PBS (140 $\left.\mathrm{mM} \mathrm{NaCl}, 2.7 \mathrm{mM} \mathrm{KCl}, 10 \mathrm{mM} \mathrm{Na}_{2} \mathrm{HPO}_{4}, 1.8 \mathrm{mM} \mathrm{KH}_{2} \mathrm{PO}_{4}\right)$ and $15 \mu 10.2 \mathrm{M} \mathrm{PMSF}, 150 \mu 1$ $10 \%$ Triton X100, $3 \mu 1$ DNAse 1, $11.25 \mu$ l Complete, $0.45 \mu 1$ Leupeptin, $4.5 \mu 1$ Pefablock and $2.7 \mu 10.5 \mathrm{M}$ EDTA were added. The cell solution was treated with the french press for three times on ice and afterwards used for coupling to the sepharose matrix (see 3.4.6.2).

\subsubsection{Coupling of GST-fusion protein to sepharose}

To couple the GST-fusion protein to the sepharose matrix, $100 \mu 150 \%$ slurry was added to the cell lysates and incubated at $4{ }^{\circ} \mathrm{C}$ for $30-50 \mathrm{~min}$ while shaking constantly. The matrix was sedimented at $500 \mathrm{~g}$ for $5 \mathrm{~min}$. The supernatant was carefully removed and the sediment was washed with $500 \mu \mathrm{l}$ PBS $\left(140 \mathrm{mM} \mathrm{NaCl}, 2.7 \mathrm{mM} \mathrm{KCl}, 10 \mathrm{mM} \mathrm{Na} 2 \mathrm{HPO}_{4}, 1.8 \mathrm{mM}\right.$ $\mathrm{KH}_{2} \mathrm{PO}_{4}$ ) three times and finally resuspended in $500 \mu 1 \mathrm{PBS}$.

\subsubsection{Native cell extracts}

For the assembly of native protein extracts, cells expressing GFP-Atg8 were prepared for preculture and the next day inoculated in main culture in a dilution of $1: 100$.

On day 3, $40 \mathrm{OD}_{600}$ of the cells were harvested and washed with $\mathrm{H}_{2} \mathrm{O}$ by centrifugation for 5 min at $4{ }^{\circ} \mathrm{C}$. The cell pellet was resupended in $1 \mathrm{ml}$ lysis buffer $(100 \mathrm{mM} \mathrm{NaCl} ; 50 \mathrm{mM}$ Tris/HCl pH 7.5; 5 mM MgCl $2 ; 1$ mM EDTA; $5 \%$ Glycerol; added freshly: 100 mM PMSF, $1000 \times$ inhibitor-mix, $25 \mathrm{x}$ Complete (Roche)), added to $0.2 \mathrm{ml}$ glass beads and vortexed 15 min at $\mathrm{V}_{\max }$ and $4{ }^{\circ} \mathrm{C}$. After this cell lysis the tube was centrifuged at $5000 \mathrm{rpm}$ for $5 \mathrm{~min}$ at 4 ${ }^{\circ} \mathrm{C}$ and the supernatant could be used for GST-pull down experiments or stored at $-80{ }^{\circ} \mathrm{C}$. 


\subsubsection{GST-Pull down}

For the GST-pull down experiment $500 \mu 1\left(20 \mathrm{OD}_{600}\right)$ of native cell extract were incubated with the prepared GST-column for $4 \mathrm{~h}$ at $4{ }^{\circ} \mathrm{C}$ while shaking. Afterwards the beads were sedimented at $500 \mathrm{~g}$ for $5 \mathrm{~min}$ at $4{ }^{\circ} \mathrm{C}$, the supernatant was removed and the GST-beads were washed three times with lysis buffer at $500 \mathrm{~g}$ for $5 \mathrm{~min}$ at $4{ }^{\circ} \mathrm{C}$. For elution of the bound proteins $200 \mu \mathrm{l}$ of elution buffer $(10 \mathrm{mM}$ red. glutathione; $50 \mathrm{mM}$ Tris/ $\mathrm{HCl} \mathrm{pH} \mathrm{8.0)} \mathrm{were}$ added and the beads were sedimented at $500 \mathrm{~g}$ for $5 \mathrm{~min}$ at $4{ }^{\circ} \mathrm{C}$ and the supernatant was taken. After repeating the elution for 2 times the eluates were pooled and precipitated using $50 \%$ TCA, incubated on ice for $20 \mathrm{~min}$ and centrifuged at $13000 \mathrm{rpm}$ for $10 \mathrm{~min}$ at $4{ }^{\circ} \mathrm{C}$. The pellet was washed with acetone, dried for a few minutes and resuspended in $50 \mu 1$ laemmli buffer. 


\section{Results}

\subsection{Synthetic Genetic Array}

The Synthetic Genetic Array analysis (SGA) is a powerful high-throughput technique for exploring synthetic lethality. It allows the systematic construction of double deletion mutants of a query strain and an entire genome deletion library. The identification of synthetic lethality highlights genes with similar function or genes with similar patterns of genetic interactions (Tong, 2001; Tong and Boone, 2006).

To ensure the preparation of the query strain, mating and sporulation steps were controlled via microscopy. Figure 15 represents the zygotes generated by mating (A) and the resulting spores generated by incubation of the diploid cells in enriched sporulation medium (B). The spores were plated on medium selecting for the haploid progeny containing the required genetic attributes. Query strains deleted in ATG8, ATG15, ATG18 and ATG21 were generated (see 3.2.11.1).

A

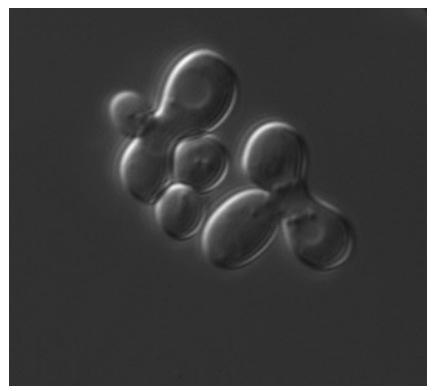

$\mathrm{B}$

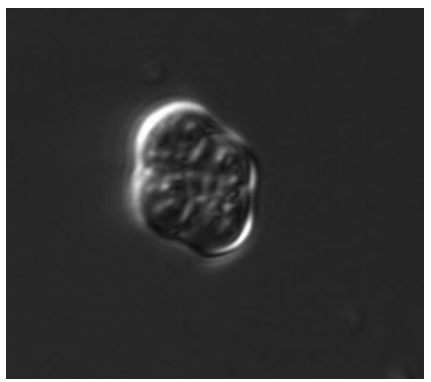

Figure 15: Mating and sporulation for query strain generation was controlled by microscopy

(A) The mating of Y8205 and the deletion strain of interest resulted in zygotes without any abnormality; (B) the incubation for 5 days in enriched sporulation medium resulted in spores of common character

All generated query strains were used for the SGA screen as described in chapter 3.2.11.2. The last step of the screen, the selection for the haploid progeny of mating type a containing the double deletion (query $\Delta:: N a t M X 4$ and $\left.x_{(D M A)} \Delta:: K a n M X 4\right)$, was arranged by the use of SD-his/arg/lys medium containing $100 \mu \mathrm{g} / \mathrm{l}$ clonNAT, $200 \mu \mathrm{g} / \mathrm{l} \mathrm{G} 418,100 \mu \mathrm{g} / \mathrm{l}$ can and $100 \mu \mathrm{g} / 1$ lyp. A total of 53 plates had to be evaluated concerning double deletions resulting in synthetic lethality. Figure 16 shows two of the OmniTray plates after the last selection step of the screen. Two colonies per double deletion were pinned on one OmniTray plate and every plate was done in duplicates. Therefore, single colonies without growth could be ignored. When both colonies, pinned on one plate, were not able to grow, the double mutant was 
nominated as synthetic lethal. The synthetic lethality was confirmed by the same growth phenotype on the second, identic control plate. All detected synthetic double deletions are listed in table 18.
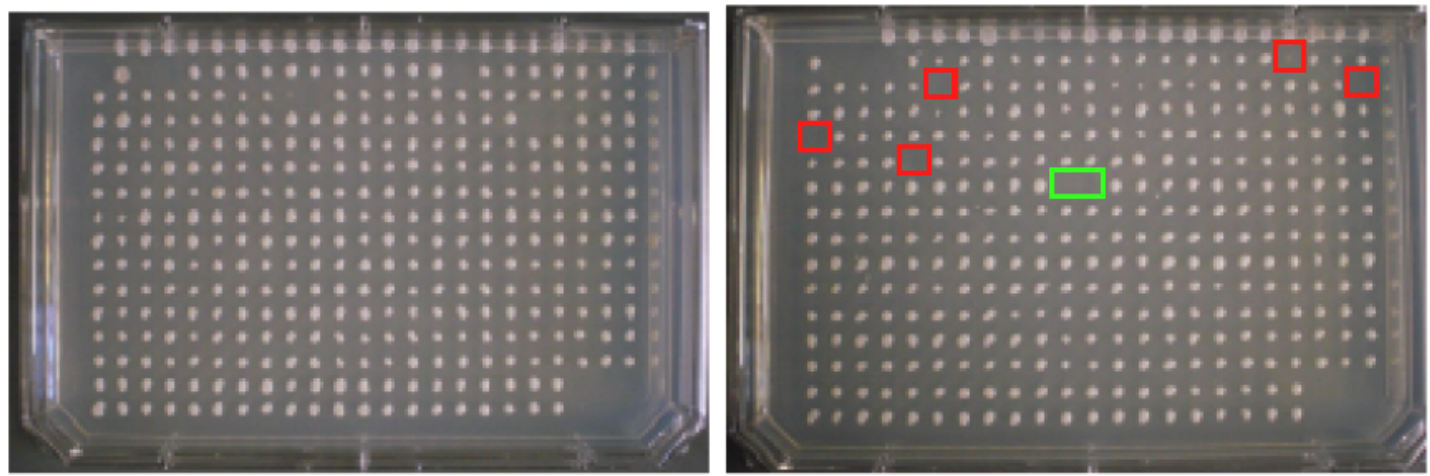

\section{Figure 16: SGA plates}

Representative OmniTray plates after the last selection step: SD-his/arg/lys medium containing $100 \mu \mathrm{g} / 1$ clonNAT, $200 \mu \mathrm{g} / 1 \mathrm{G} 418100 \mu \mathrm{g} / 1 \mathrm{can}$ and $100 \mu \mathrm{g} / 1 \mathrm{lyp}$, resulting in haploid progeny of mating type a with double deletion (query $\Delta::$ NatMX 4 and $\left.x_{(D M A)} \Delta:: \mathrm{KanMX} 4\right)$; on the right: single colonies without growth are marked with a red box, both colonies of one double deletion mutant defective in growth are marked with a green box.

Table 18: Strains occurring as synthetic lethal in SGA

\begin{tabular}{|c|c|c|}
\hline $\begin{array}{c}\text { query strain } \\
\text { query } \triangle: \text { NatMX4 }\end{array}$ & $\begin{array}{c}\text { DMA strain } \\
x_{(D M A)} \Delta:: \operatorname{KanMX} 4\end{array}$ & \\
\hline \multirow[t]{13}{*}{$\operatorname{atg} 15 \Delta$} & YDR234W & $\operatorname{lys} 4 \Delta$ \\
\hline & YOR302W & yor $302 w \Delta$ \\
\hline & YOR303W & Ccals \\
\hline & YOL058W & $\arg 1 \Delta$ \\
\hline & YOR323c & pro2s \\
\hline & YOR258W & $h n t 3 \Delta$ \\
\hline & YGL033W & hop $2 \Delta$ \\
\hline & YIL094c & $\operatorname{lys} 12 \Delta$ \\
\hline & YEL057c & yel057cd \\
\hline & YCR010c & $\operatorname{ady} 2 \Delta$ \\
\hline & YPL125W & 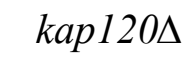 \\
\hline & YNL053W & $m s g 5 \Delta$ \\
\hline & YMR143W & $\operatorname{rps} 16 A \Delta$ \\
\hline \multirow[t]{3}{*}{$\operatorname{atg} 18 \Delta$} & YMR280c & cat8A \\
\hline & YNL242W & $\operatorname{atg} 2 \Delta$ \\
\hline & YJL122W & alb1 $\Delta$ \\
\hline \multirow[t]{2}{*}{$\operatorname{atg} 8 \Delta$} & YOL127c & hmols \\
\hline & YLL0026W & hsp 104A \\
\hline \multirow[t]{2}{*}{$\operatorname{atg} 21 \Delta$} & YDR323c & vac1 $\Delta$ \\
\hline & YJL178c & $\operatorname{atg} 27 \Delta$ \\
\hline
\end{tabular}


For confirmation of the synthetic lethality of the double deletion $\operatorname{atg} 21 \Delta \operatorname{vac} 1 \Delta$ and $\operatorname{atg} 21 \Delta$ $\operatorname{atg} 27 \Delta$ in the screen (Table 18; bold), these double deletions were generated again in a separate experiment.

The double mutants were generated by conventional mating (see 3.2.5), sporulation (see 3.2.8) and selection steps, using media similar to the SGA-screen (see 3.2.11.2). Both double deletion combinations presented neither a synthetic lethality nor a defect in growth of colonies on SD-his/arg/lys medium containing $100 \mu \mathrm{g} / \mathrm{l}$ clonNAT, $200 \mu \mathrm{g} / \mathrm{l} \mathrm{G} 418,100 \mu \mathrm{g} / \mathrm{l}$ can and $100 \mu \mathrm{g} / \mathrm{l}$ lyp, selecting for the haploid progeny of mating type a (query $\Delta:$ NatMX4 $\left.x_{(D M A)} \Delta:: K a n M X 4\right)$.

For further control of the $\operatorname{atg} 21 \Delta \operatorname{atg} 27 \Delta$ double deletion in a second yeast background, $\operatorname{atg} 27 \Delta::$ HIS3 cells of the WCG background were also mated with $\operatorname{atg} 21 \Delta:: \mathrm{KAN}$ of the WCG background. The resulting diploids were sporulated (see 3.2.8), tetrads were separated (see 3.2.9) and examined for growth ability on medium lacking histidine and containing $200 \mu \mathrm{g} / 1$ G418. The tetrad separation of these diploid double mutant resulted in the growth of four different haploid cells, including the double deletion ( $\operatorname{atg} 21 \Delta:: \mathrm{KAN}$ atg27 $::$ HIS3) without any viability or growth defect. 


\subsection{Detection of nonselective bulk autophagy using Pgk1-GFP}

For the detection of autophagy by western blot, GFP-Atg8 is the common marker protein. In this study the cytosolic 3-phosphoglycerate kinase Pgk1 could be introduced as novel marker protein (Welter, 2010). To detect the autophagic degradation of Pgk1-GFP, the plasmid pRS316-Pgk1-GFP was constructed in the Thumm laboratory and used for experiments in this study.

Cells expressing Pgk1-GFP were starved up to 6 hours in SD-N medium and samples were taken at defined time points $(0,2,4$ and $6 \mathrm{~h})$. The cells were alkaline lysed and treated for immunoblotting using anti-GFP antibody, as also described in Welter et al. (2010). Upon delivery of Pgk1-GFP into the vacuole, Pgk1 is rapidly degraded, whereas the released GFP remains relatively stable. Accordingly, the appearance of free GFP can be used to monitor unselective autophagy.

In wild type cells a clear signal for free GFP was detected. ATG21 deleted cells showed a reduction in signal quantity compared to wild type cells. ATG1 deleted cells, deficient in autophagy, and pep $4 \Delta$ cells, deficient in intravacuolar proteolysis, were used as controls (Figure $17(\mathrm{~A})$ ). The signal intensities of the western blot bands were quantified using AIDA software. The ratio between free GFP and Pgk1-GFP was calculated, taking wild type cells (6 h) as $100 \%$. The resulting values of three independent experiments are displayed in figure 17 (B). Figure 17 (C) highlights the values after 6 hours of starvation. 


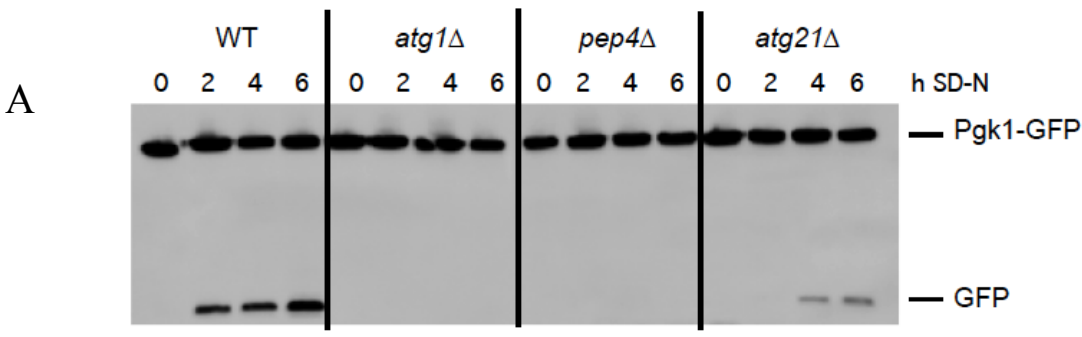

B

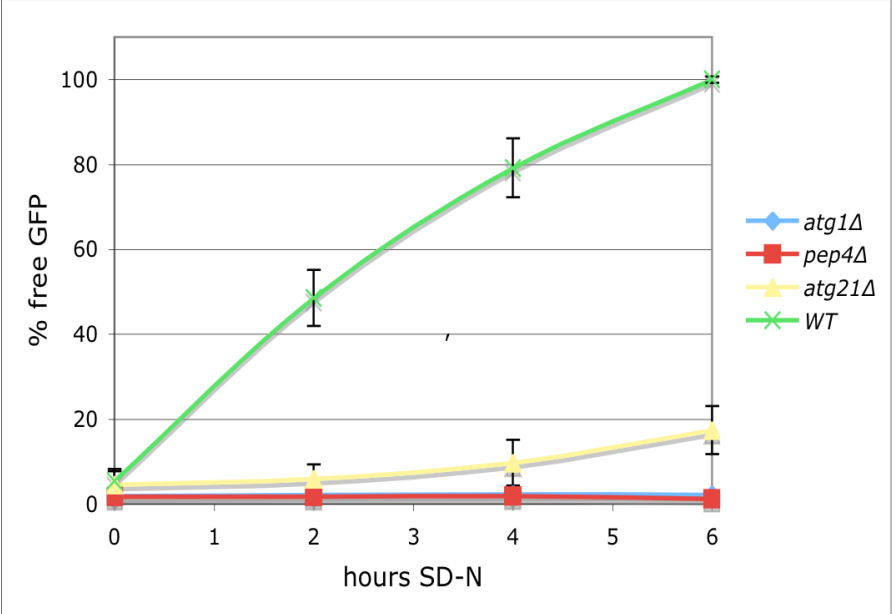

$\mathrm{C}$

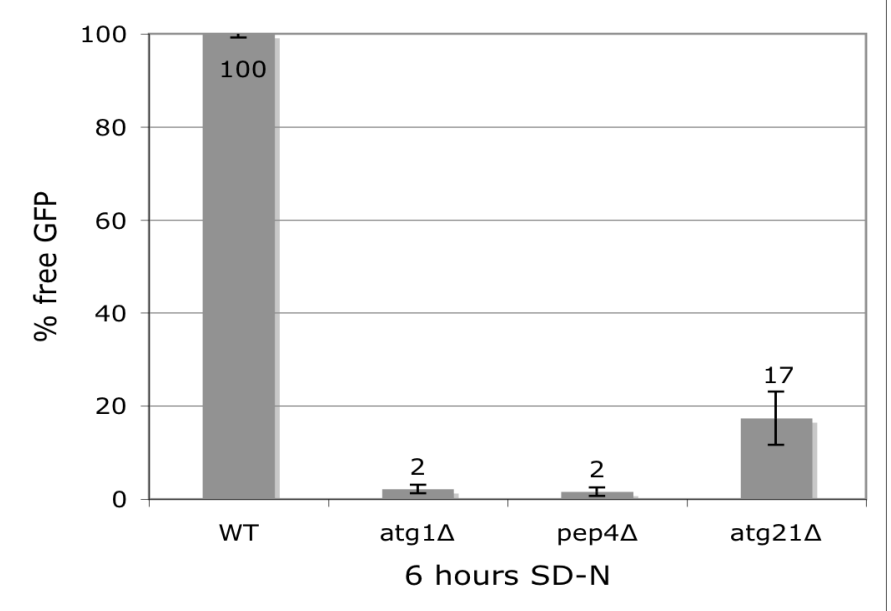

Figure 17: Monitoring unselective autophagy using Pgk1-GFP

(A) Wild type (WT), atg $1 \Delta$, pep $4 \Delta$ and atg2 $1 \Delta$ cells expressing Pgk1-GFP were starved in SD-N, samples were alkaline lysed and immunoblotted. Using a monoclonal mouse anti-GFP antibody and a peroxidase-coupled secondary antibody, Pgk1-GFP $(\sim 71 \mathrm{kDa})$ and free GFP $(\sim 26 \mathrm{kDa})$ were detected. (B) Quantification of signal intensities by AIDA software. The value of wild type cells starved for 6 hours was set to $100 \%(\mathrm{n}=3)$.

(C) Values of free GFP of cells starved for 6 hours are presented. 
The degradation of Pgk1-GFP was also shown in fluorescence microscopy experiments (Figure 18). Wild type, atg $1 \Delta$, pep $4 \Delta$ and $\operatorname{atg} 21 \Delta$ cells expressing Pgk1-GFP were starved for 4 hours in SD-N medium containing $1 \mathrm{mM}$ PMSF, to avoid vacuolar proteolysis. Using a DeltaVision spectris fluorescence microscope equipped with a FITC filter, a high amount of

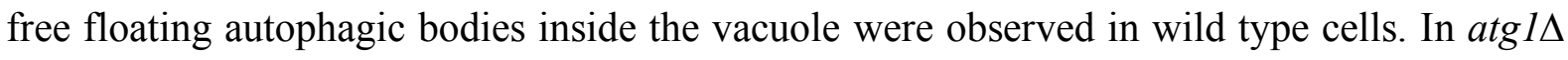
cells, used as negative control, no autophagic bodies appeared in the vacuole, while in pep $4 \Delta$ cells, unable for degradation of vacuolar vesicles, a clear accumulation of autophagic bodies was observed. A small amount of green vesicles was found in atg21 $1 \Delta$ cells, supporting the observation in the western blot analysis.

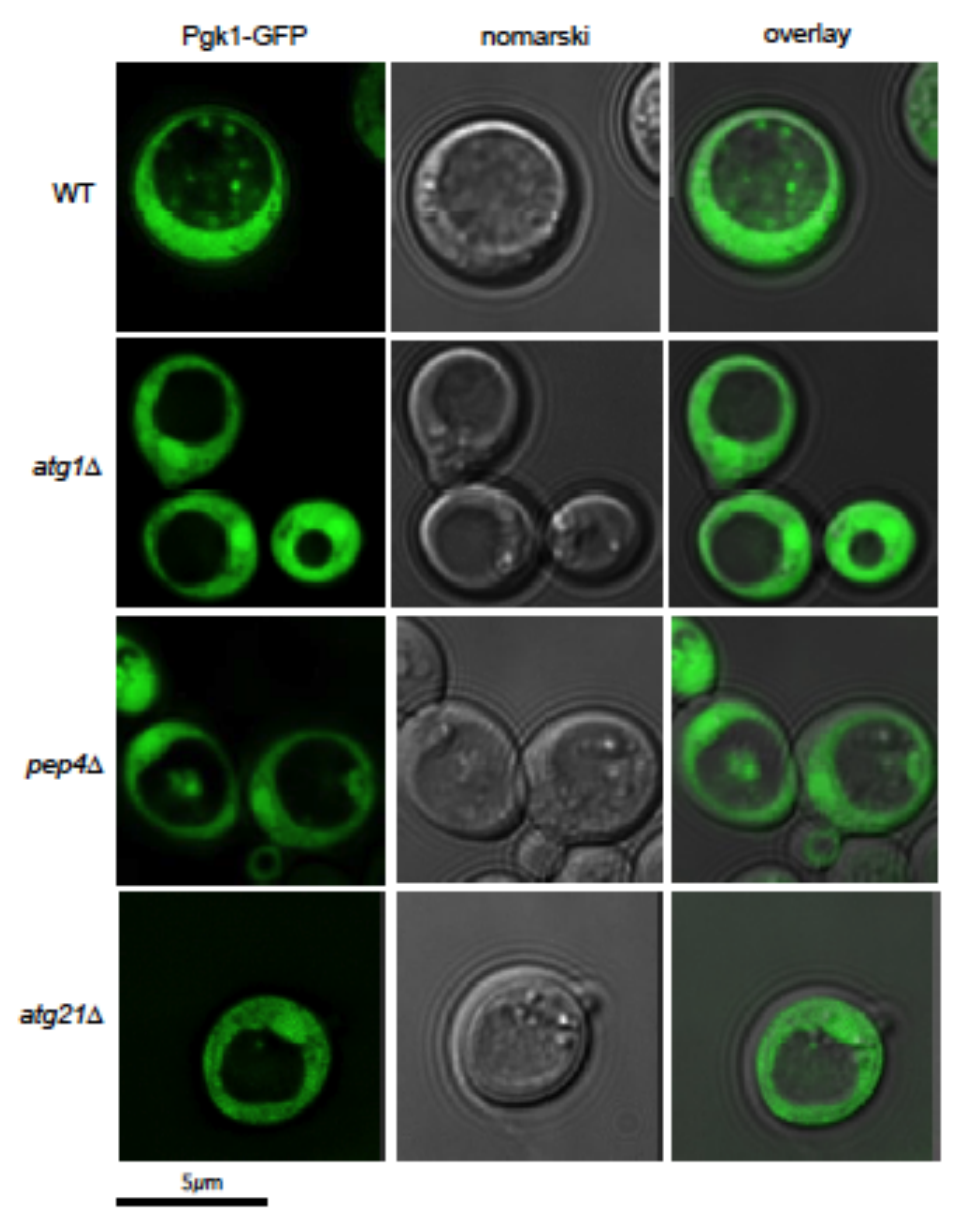

Figure 18: Fluorescence microscopy of cells expressing Pgk1-GFP

Wild type (WT), atg1 $1 \Delta$, pep $4 \Delta$ and atg $21 \Delta$ cells (WCG) expressing Pgk1-GFP were starved for $4 \mathrm{~h}$ in SD-N medium containing $1 \mathrm{mM}$ PMSF. The cells were observed by fluorescence microscopy using a DeltaVision microscope (FITC filter) and the accumulation of autophagic bodies in the vacuole was analysed. 


\subsection{Cdc48 and its cofactor Shp1 are needed for functional autophagy}

Cdc48 and Shp1 (also termed Ubx1) are required for autophagosome biogenesis (Krick, 2010).

\subsubsection{Shp1 is the exclusive cofactor of Cdc48 needed for autophagy}

The requirement of Cdc48 for autophagy was shown by the use of the standard GFP-Atg8 degradation assay (see 3.4.5.1) (Bremer, 2009).

Cdc48 is known to be involved in different pathways (ERAD, UFD). Caused by this diversity in function, many cofactors of Cdc48 have been identified (overview see (Buchberger, 2010). To elucidate the need for autophagy and to confirm former results (Bremer, 2009), strains deleted in these cofactors and regulators were tested for functional autophagy.

Members of the UBX domain protein family are known to act as Ccd48 regulators (Schuberth, 2004). Therefore $U b x 1$ to $U b x 6$ deletion strains were tested in the standard GFPAtg8 degradation assay for their involvement in autophagy (see 3.4.5.1).

After 4 hours of starvation in SD-N medium, decreasing signal for GFP-Atg8 and increasing signal of free GFP was detectable in wild type cells, whereas in the negative control atgl $1 \Delta$, no free GFP was detected. Furthermore $u b x 1 \Delta(\operatorname{shp} 1 \Delta)$ cells showed a clear reduction of free GFP in contrast to the other deletion strains of the UBX protein family $u b x 2 \Delta, u b x 3 \Delta, u b x 4 \Delta$, $u b x 5 \Delta$ and $u b x 6 \Delta$.

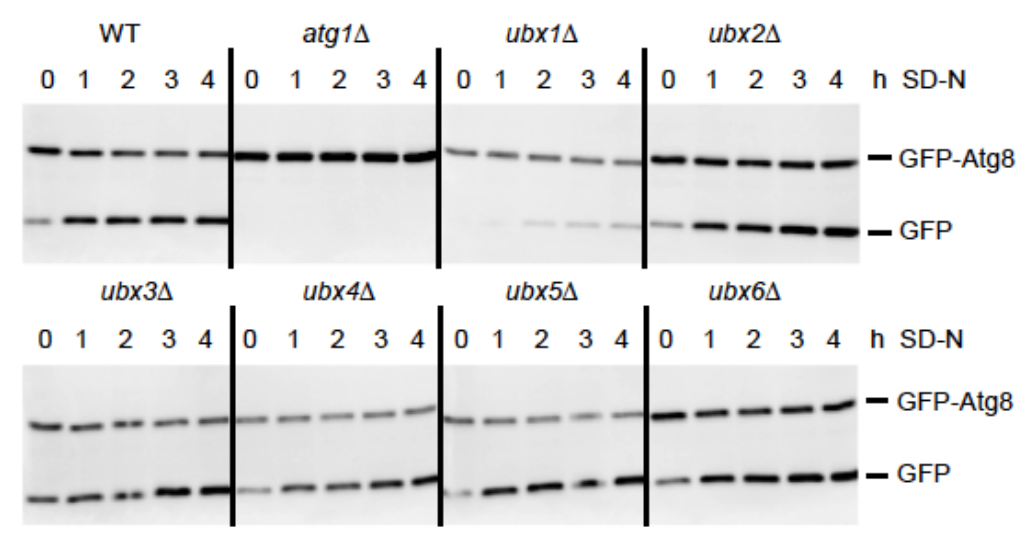

Figure 19: Ubx1 but no other UBX domain protein is required for autophagy

Wild type (WT), atg $1 \Delta$ and $u b x 1 \Delta-u b x 6 \Delta$ cells expressing GFP-Atg8 were starved in SD-N, samples were alkaline lysed and immunoblotted. Using a monoclonal mouse anti-GFP antibody, GFP-Atg8 ( 39 $\mathrm{kDa})$ and free GFP $(\sim 26 \mathrm{kDa})$ were detected. 
In the next step, it was analysed if the ubiquitin-proteasome system, represented by the substrate-processing Cdc48 cofactors Ufd2 and Ufd3, the ubiquitin ligase Ufd4 and the proteasome regulator $\mathrm{Ufd} 5$, is required for macroautophagy.

As Ufd1, a crucial substrate-recruiting cofactor, is an essential protein, a strain containing a point-mutation in the UFD1 allele (ufd1-1) was used for GFP-Atg8 degradation assay experiments (Figure 20 (B)). Ufd1-1 and all further tested strains, deleted in one of the

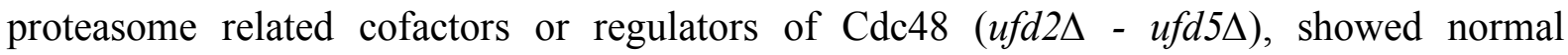
starvation induced degradation of GFP-Atg8, comparable to wild type cells (Figure 20 (A)).

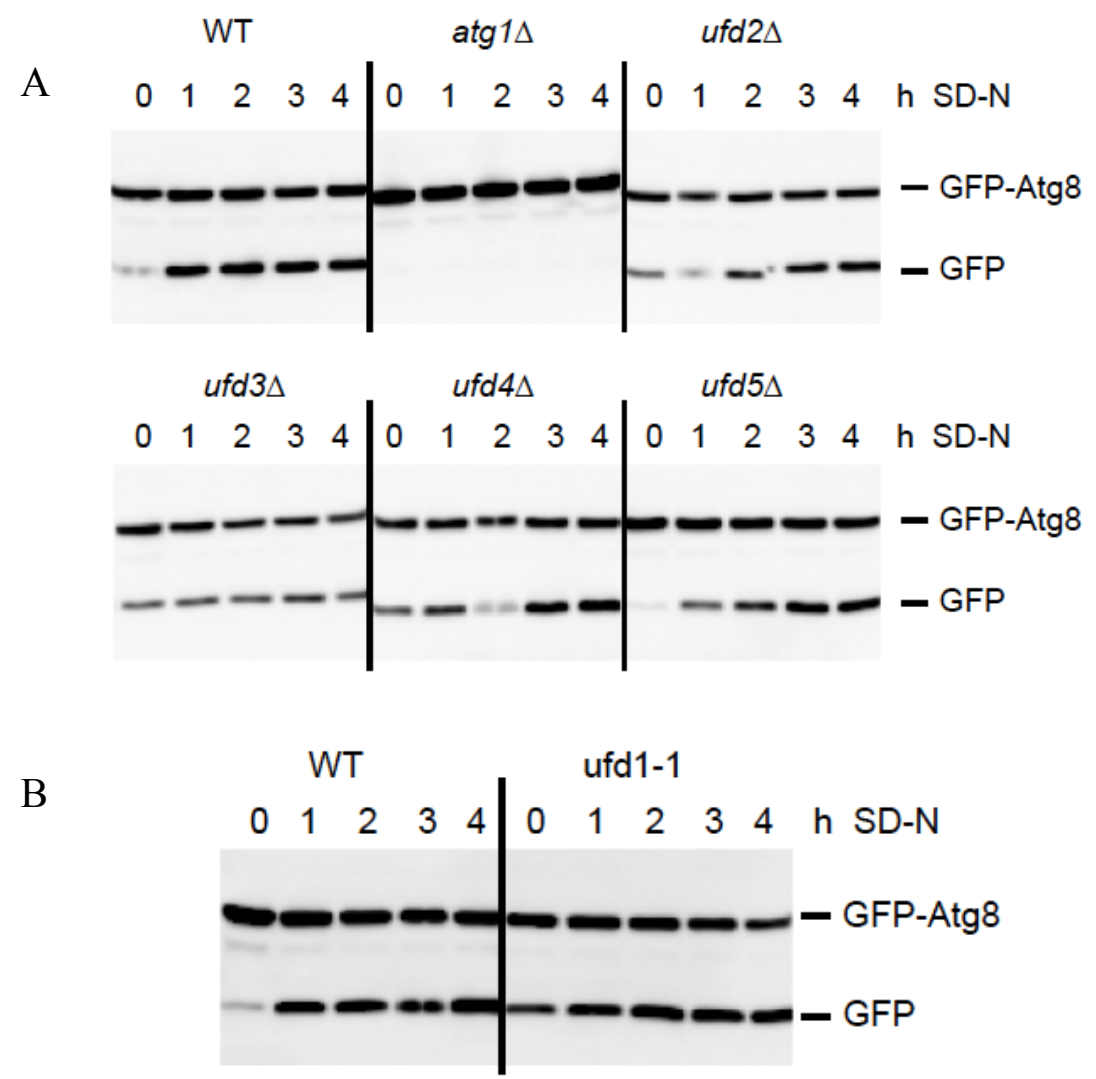

Figure 20: Ufd proteins are not required for autophagy

(A) Wild type (WT), $\operatorname{atg} 1 \Delta$ and $u f d 2 \Delta-u f d 5 \Delta$ cells, (B) wild type and ufd1-1 cells expressing GFPAtg8 were starved in SD-N, samples were alkaline lysed and immunoblotted. Using a monoclonal mouse anti-GFP antibody, GFP-Atg8 ( $\sim 39 \mathrm{kDa})$ and free GFP $(\sim 26 \mathrm{kDa})$ were detected.

Furthermore the ERAD component Der1 and the Cdc48 substrate-processing cofactor Otu1 were tested for intact autophagy.

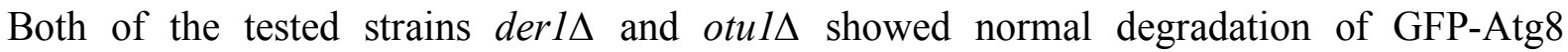
comparable to wild type cells, while in the negative control atg1 $1 \Delta$ no signal for GFP was detected (Figure 21). 


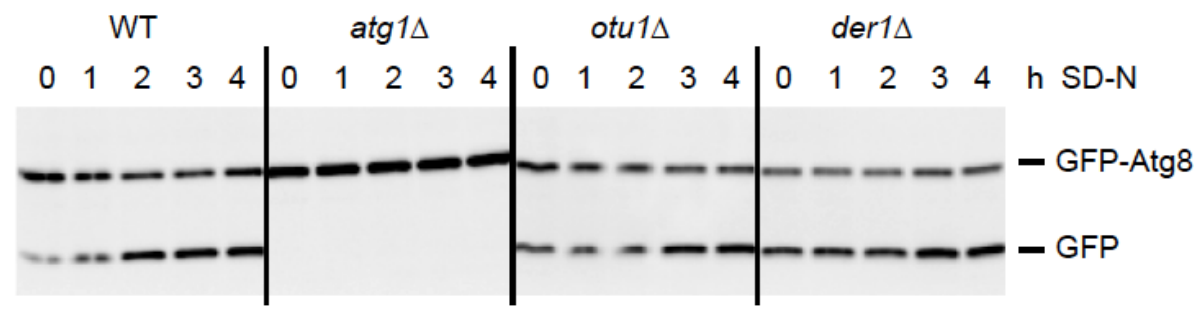

Figure 21: Otu1 and Der1 are not required for autophagy

Wild type (WT), atg $1 \Delta$, der $1 \Delta$ and otu $1 \Delta$ cells expressing GFP-Atg8 were starved in SD-N, samples were alkaline lysed and immunoblotted. Using a monoclonal mouse anti-GFP antibody, GFP-Atg8 ( 39 $\mathrm{kDa})$ and free GFP $(\sim 26 \mathrm{kDa})$ were detected.

Furthermore Doa4 was tested for the involvement in autophagy. Doa4 is an ubiquitin isopeptidase, required for recycling of ubiquitin from proteasome-bound polyubiquitinated proteins. The deletion of DOA4 depletes the cellular ubiquitin level (Swaminathan, 1999; Ren, 2008).

The WCG doa4d strain was generated by homologous recombination in this thesis (see 3.3.13) and the cells were used for GFP-Atg8 degradation assays. These assays resulted in the detection of free GFP signal in doa $4 \Delta$ cells, comparable to wild type cells.

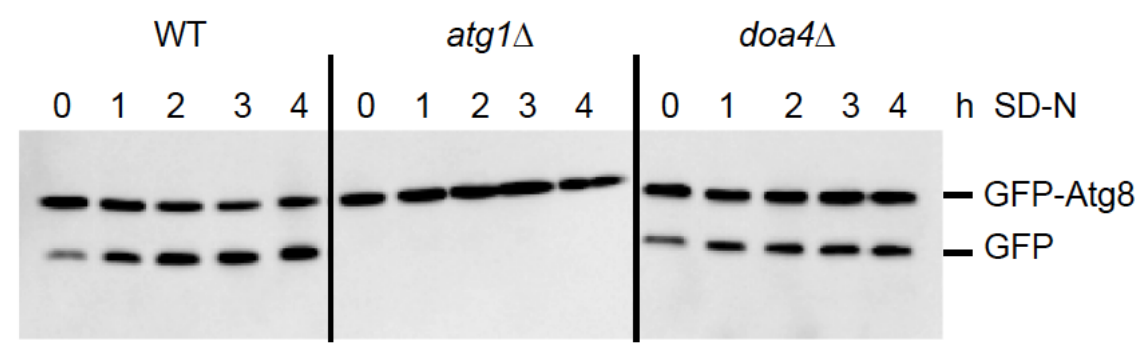

Figure 22: Macroautophagy is not affected in $\operatorname{doa} 4 \Delta$ cells

Wild type (WT), atg $1 \Delta$ and doa4 $\Delta$ cells (WCG) expressing GFP-Atg8 were starved in SD-N, samples were alkaline lysed and immunoblotted. Using a monoclonal mouse anti-GFP antibody, GFP-Atg8 ( 39 $\mathrm{kDa})$ and free GFP $(\sim 26 \mathrm{kDa})$ were detected. 


\subsubsection{Shp1 is needed for autophagy but not for the Cvt pathway and CPY maturation}

As an additional method, the involvement of Ubx1/Shp1 in autophagy was tested in a degradation assay using Pgk1-GFP as marker protein (see 4.2) (Welter, 2010).

Wild type, atgl $\Delta$ and shpl $\Delta$ cells were transformed with Pgk1-GFP. Stationary grown cells were starved in SD-N up to 6 hours, samples were taken $(0,2,4$ and $6 \mathrm{~h})$, treated for immunoblot as previously described and visualised using a monoclonal mouse anti-GFP antibody. In this Pgk1-GFP degradation assay Pgk1-GFP and free GFP were detected in wild type cells. In contrast, shpl $\Delta$ and atg $1 \Delta$ cells showed no signal for free GFP.

Furthermore the blots were reprobed with a polyclonal rabbit anti-ApeI antibody to detect a potential dysfunction in the Cvt-pathway. However, the maturation of aminopeptidase 1

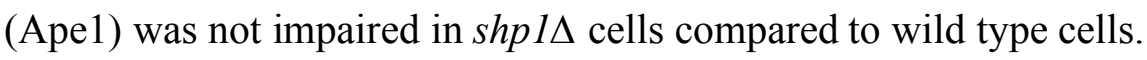

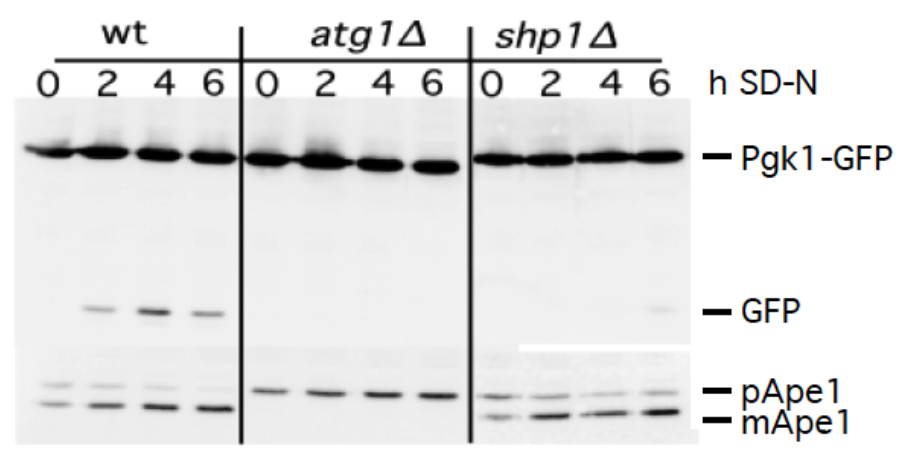

Figure 23: Shp1 is required for autophagy but not for the Cvt-pathway

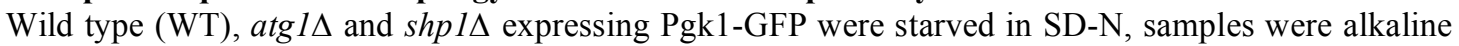
lysed and immunoblotted. Using a monoclonal mouse anti-GFP antibody, Pgk1-GFP ( 71 kDa) and free GFP ( 26 kDa) were detected, prApel $(\sim 62 \mathrm{kDa})$ and mApe1 $(\sim 57 \mathrm{kDa})$ were detected using a polyclonal rabbit anti-Ape1 antibody.

Ape1 can be matured either selectively via the Cvt pathway or unselectively via autophagy, dependent of the growth phase and supply of nutrients. Also the carboxypeptidase Y (CPY) is synthesised as a prepro-form and travels through the ER and Golgi to the vacuole in an autophagy-independent manner (Conibear and Stevens, 1998).

To test the involvement of Shp1 in these pathways, wild type, $\operatorname{shp} 1 \Delta, \operatorname{atg} 1 \Delta, \operatorname{atg} 21 \Delta$ and pep $4 \Delta$ cells of different growth phases (logarithmic $(\log )$ and stationary (stat)) and starved cells were tested for maturation of Ape1 and CPY.

Figure 24 shows, that the maturation of Ape1 and CPY in shpls cells occurs comparable to wild type cells. The control strains atg $1 \Delta$ and pep $4 \Delta$ are blocked in maturation of Ape1 and 
pep $4 \Delta$ cells are also blocked in maturation of CPY. In atg $21 \Delta$ cells, where the Cvt pathway is blocked, the maturation of Ape1 was only observed under starvation conditions.

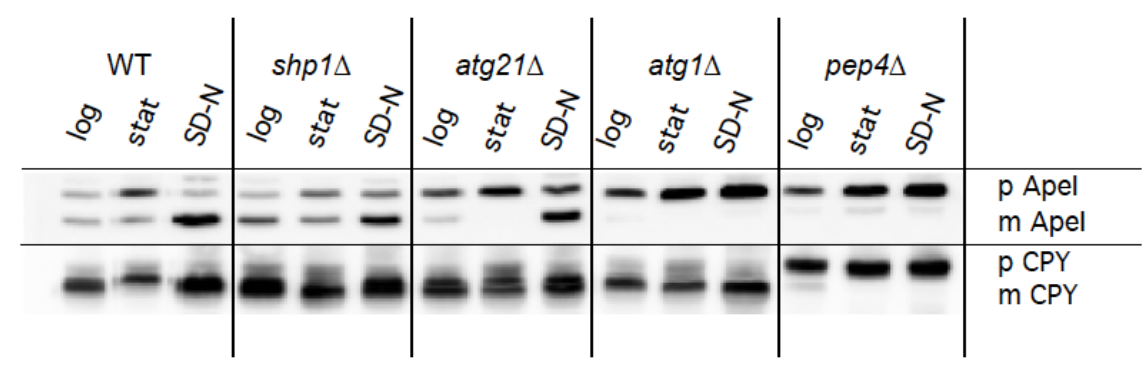

Figure 24: The maturation of Ape1 and CPY occurs normal in shp1 $\Delta$ cells Samples of wild type (WT), $\operatorname{shp} 1 \Delta, \operatorname{atg} 1 \Delta$, atg $21 \Delta$ and pep $4 \Delta$ cells in logarithmic and stationary growth phase and starved in SD-N medium were taken, alkaline lysed and immunoblotted. prApe1 ( $\sim 62 \mathrm{kDa})$ and mApe1 $(\sim 57 \mathrm{kDa})$ were detected using a rabbit anti-Apel antibody and pCPY $(\sim 69 \mathrm{kDa})$ and mCPY $(\sim 61 \mathrm{kDa})$ were detected using a mouse anti-CPY antibody.

\subsubsection{No accumulation of autophagic bodies occurs in $\operatorname{sh} 1 \Delta$ cells}

Another standard method in the field of autophagy is the visualisation of intravacuolar

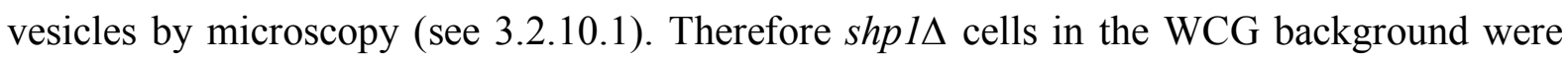

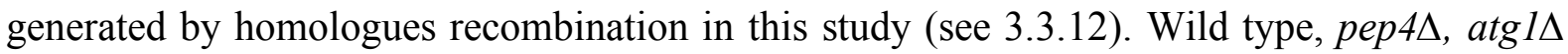
and $\operatorname{shp} 1 \Delta$ cells of WCG background were starved in SD-N medium. Additional cultures of wild type, pep $4 \Delta, \operatorname{atg} 1 \Delta$ and $\operatorname{shp} 1 \Delta$ cells were treated with $1 \mathrm{mM}$ PMSF to inhibit vacuolar proteinase B. The cells were used for microscopy (see 3.2.10.1). Distinct to other vesicles, also transported to the vacuole (e.g. MVB vesicles), autophagic bodies can be observed as little swirling dots in the vacuole.

Figure 25 (A) displays yeast cells after starvation for 4 hours in SD-N. Wild type cells treated with $1 \mathrm{mM}$ PMSF showed various autophagic bodies inside the vacuole, cells without PMSF exhibited an empty vacuole caused by the degradation of autophagic bodies by vacuolar proteases. The positive control pep $4 \Delta$, defective for the breakdown of vesicles, showed an intense accumulation of autophagic bodies. In contrast, the autophagy deficient control cells atg1D showed no vesicles inside the vacuole. SHP1 deleted cells also showed no accumulation of autophagic bodies in the vacuole, even not with PMSF treatment. For statistic interpretation 200 cells of each strain (with and without $1 \mathrm{mM}$ PMSF) were monitored and evaluated concerning the accumulation of vesicles (Figure 25 (B)). 
A

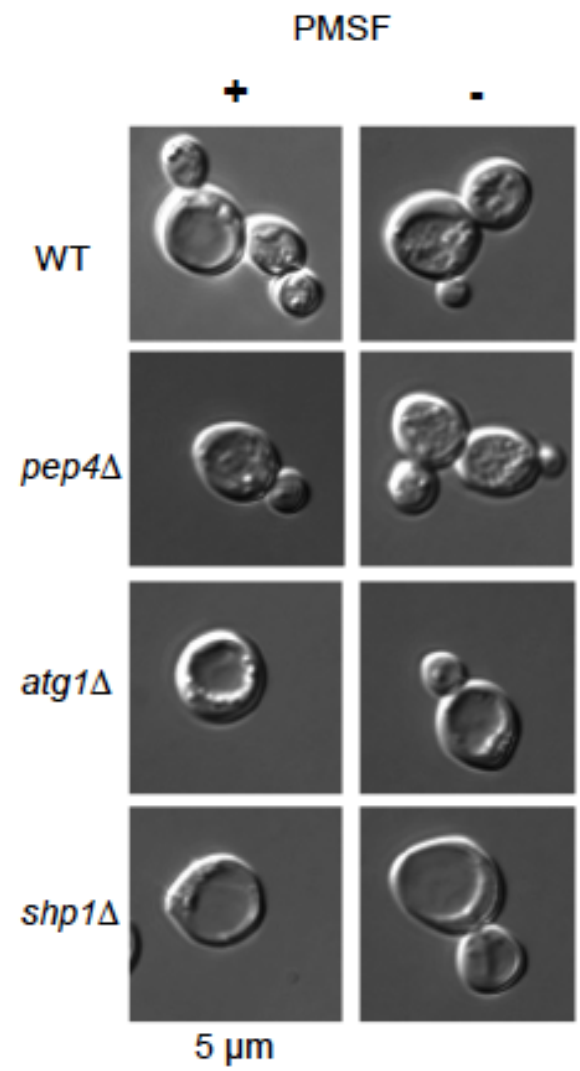

B

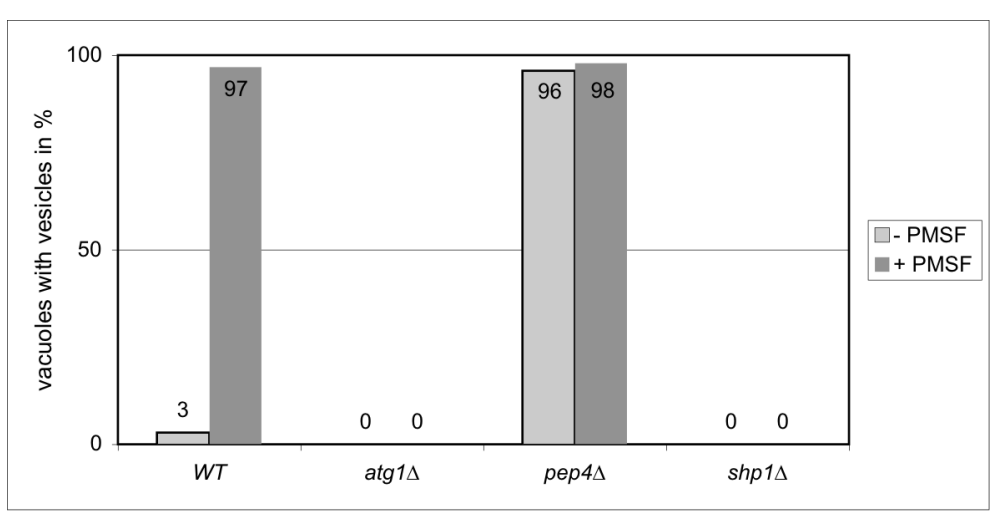

Figure 25: No vacuolar accumulation of autophagic bodies in shp1 $\Delta$ cells

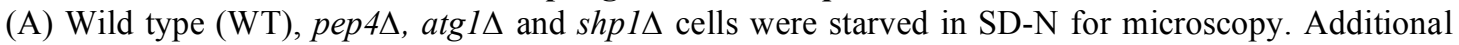
cultures of wild type and shp1 $\Delta$ cells were treated with $1 \mathrm{mM}$ PMSF to inhibit vacuolar proteases.

(B) Wild type (WT), atg $1 \Delta$, pep $4 \Delta$ and $\operatorname{shp} 1 \Delta$ cells (treated/ not treated with $1 \mathrm{mM}$ PMSF) containing autophagic bodies in the vacuole after starvation for 4 hours in SD-N were counted and evaluated.

\subsubsection{The formation of the PAS occurs normal in shp1 $1 \Delta$ cells}

A very important step during autophagy is the formation of the pre-autophagosomal structure (PAS), where the autophagic core machinery assembles (Suzuki, 2001; Kim, 2002). Besides Atg8, which is integrated via PE into the membrane during autophagosome formation, also the transmembrane protein Atg9 is known to be involved in autophagosome biogenesis. 
Therefore both proteins are qualified marker proteins for the PAS in microscopy (Reggiori, 2004a; Reggiori, 2005; Klionsky, 2007).

The formation of the PAS was observed in shpls cells in fluorescence microscopy using GFP-Atg8 (Figure 26 (A)). For statistic evaluation, cells containing clear green dots, representing the PAS, were counted. 200 cells either expressing GFP-Atg8 or GFP-Atg9 were analysed. Two independent experiments were done $(\mathrm{n}=2)$. SHPI deleted cells showed no reduction in PAS formation compared to wild type cells using both marker proteins (Figure $26(\mathrm{~B}))$.

A

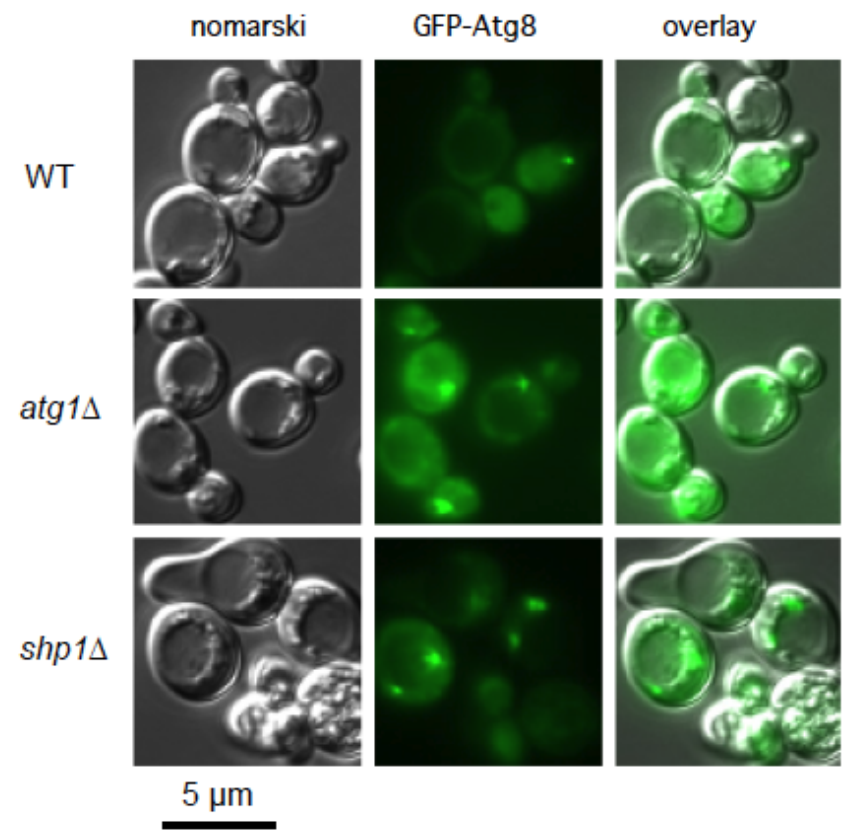

B

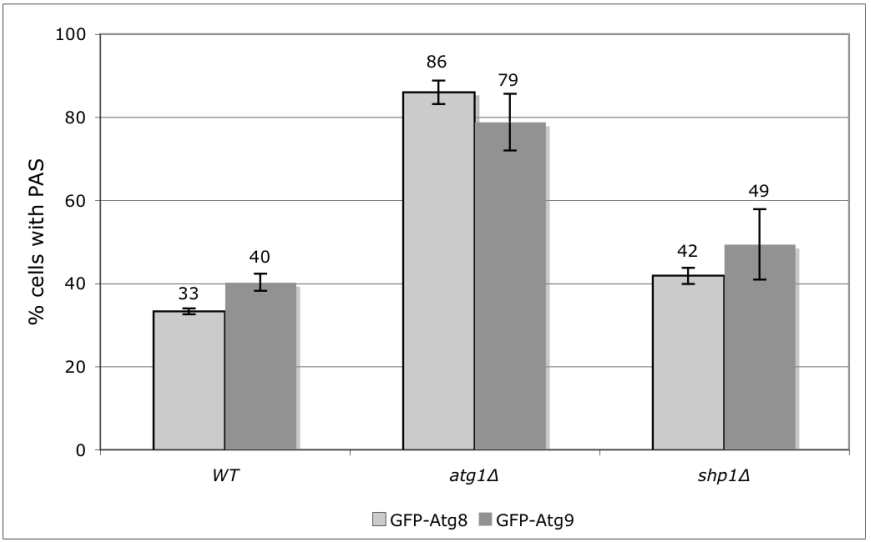

Figure 26: The formation of the PAS occurs normal in $\operatorname{shp1} \Delta$ cells

(A) Wild type (WT), atg $1 \Delta$ and shp $1 \Delta$ cells expressing GFP-Atg8 were starved in SD-N medium. The PAS represented by GFP-Atg8 was visualised in fluorescence microscopy

(B) Wild type (WT), atg $1 \Delta$ and $\operatorname{shp} 1 \Delta$ cells expressing GFP-Atg8 or GFP-Atg9 containing a clear green PAS dot were counted. 200 cells were counted in two independent experiments $(n=2)$ 


\subsubsection{Lipidation of Atg8 occurs normal in shpls cells}

Atg8 is conjugated via an ubiquitin like conjugation system to phosphatidylethanolamine (PE) (Kirisako, 2000) (see 2.2.1.2). This lipidation and also the delipidation are necessary for

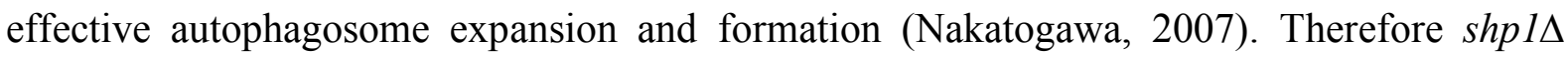
cells were tested for Atg8 lipidation in western blot analysis.

Comparable to wild type cells, the lipidation occurred slightly retarded in shp $1 \Delta$ cells, similar to atg2 $1 \Delta$ cells. ATG7 deleted cells, unable for Atg8-conjugation (Ichimura, 2000; Kirisako, 2000), showed no lipidated Atg8. The control strain atg8 showed no signal at all.

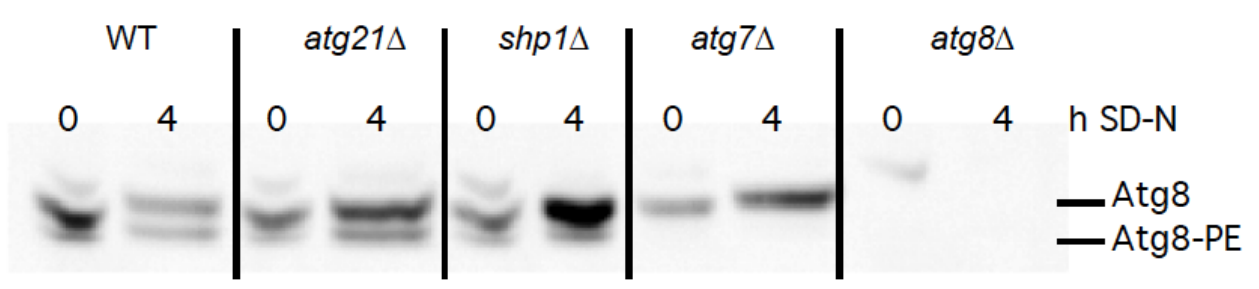

Figure 27: The lipidation of Atg8 occurs normally in $\operatorname{shp1} \Delta$ cells

Wild type (WT), $\operatorname{atg} 21 \Delta, \operatorname{shp} 1 \Delta, \operatorname{atg} 7 \Delta$ and $\operatorname{atg} 8 \Delta$ cells were starved for 4 hours in SD-N. Samples were taken after 0 and 6 hours of starvation, alkaline lysed and immunoblotted. Using a monoclonal rabbit anti-Atg8 antibody, $\operatorname{Atg} 8(\sim 14 \mathrm{kDa})$ and Atg8-PE were detected. To ensure an adequate separation of the signals, a $12 \%$ SDS gel containing 6 M Urea was used.

\subsubsection{Pull down experiments of recombinant GST-Shp1}

A split ubiquitin assay gave first incidence for a potential interaction between Shp1 and Atg8.

This interaction has been proven by coimmunoprecipitation and pull down experiments using recombinant GST-Atg8 (Krick, 2010). For further investigations, pull down experiments using recombinant GST-Shp1 were done in this study. Shp1 is known to possess an Nterminal UBA and a C-terminal UBX domain (see 2.2.5.1). Therefore full length GST-Shp1, as well as a GST-Shp1 construct lacking the UBX domain and a GST-Shp1 construct lacking the UBA domain, were generated. The three GST-fusion proteins were purified (see 3.4.6.1) and used for pull down experiments by incubation with crude extract of cells expressing GFP$\operatorname{Atg} 8$ (see 3.4.6.4).

The purification of recombinant GST-Shp1 was optimised by the use of E. coli BL21 RIPL cells for expression and a following cell disruption by french press. Figure 28 (A) exhibits samples of $E$. coli BL21 RIPL cells ahead of treatment with IPTG (1), samples of E. coli cell 
suspension before cell disruption by french press (2), samples taken after cell disruption (3) and samples of GST-coupled sepharose beads (4).

For pull down, crude cell extracts (L) from either pep4s cells expressing GFP-Atg8 (B) or $\operatorname{atg} 4 \Delta \operatorname{atg} 8 \Delta$ cells expressing GFP-Atg8-FG (C) (see 5.3.2) were incubated with GST-Shp1/UBX/-UBA and GST coupled sepharose beads. After incubation, the supernatant (S) was discarded and the beads were washed four times (W). By addition of Laemmli buffer, the GST-fusions and the interacting proteins of the crude cell extract were solubilised (PD). Samples were loaded on SDS-PAGE and immunoblotted. The resulting immunoblots incubated with GFP antibody showed a clear signal for GFP-Atg8/-FG in lysate $(\mathrm{L}=2.5 \%$ input) and supernatant (S), but not in a sample taken from the last washing step (W). Furthermore, a clear signal for GFP-Atg8/-FG could be detected in the pull down samples (PD) of each GST-Shp1 construct, but not in the negative control using only GST (Figure 28 (B) and (C)).

A

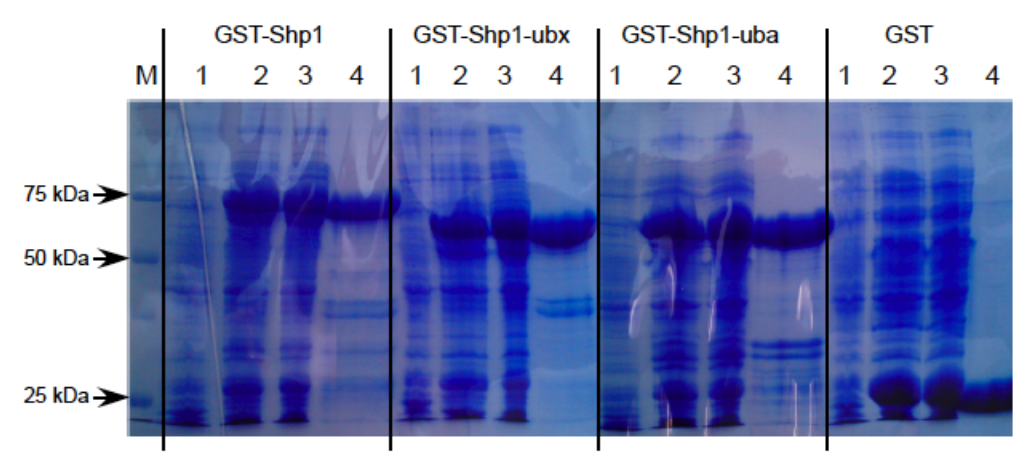

B

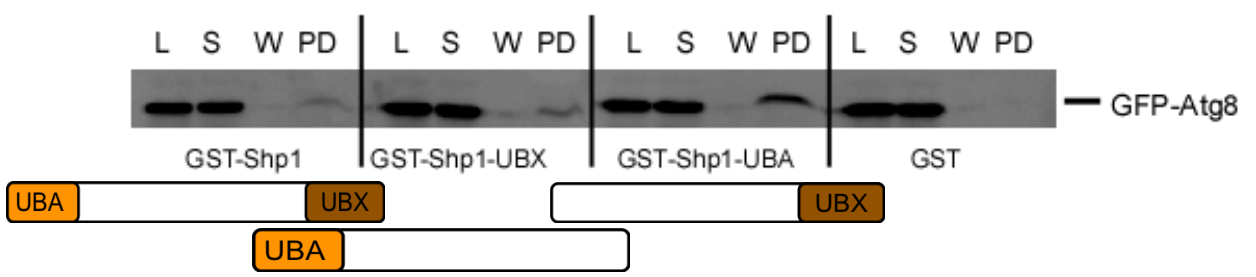

$\mathrm{C}$

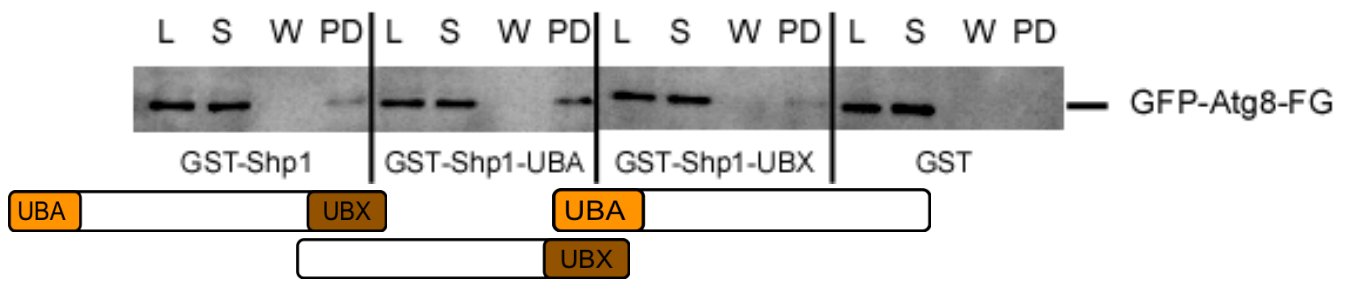

Figure 28: Pull down experiments with recombinant GST-Shp1

(A) Purification of GST-Shp1 constructs and coupling to Sepharose beads was monitored by SDSPAGE: Precision Plus Protein All Blue Standard (M); BL21 RIPL ahead of IPTG addtition (1); BL21 RIPL suspension used for cell disruption by French Press (2); extract after cell disruption (3) and fraction of GST-linked sepharose beads (4).

(B) (C) $2.5 \%$ of the crude cell lysate was loaded (L), the GST fusion proteins were incubated with lysates from WCG pep $4 \Delta$ cells expressing GFP-Atg8 (A) or WCG atg $4 \Delta$ atg8 cells expressing GFPAtg8-FG (B), supernatant (S), wash (W) and pull down (PD) were used for immuno-blotting. Monoclonal mouse anti-GFP antibody was used for detection. 


\subsection{Mitophagy in S. cerevisiae}

Mitochondria play a significant role in a lot of important metabolic processes, ranging from energy production to cell death. As a consequence, they are at the same time the major source of cellular reactive oxygen species (ROS), which cause oxidative damage to, for instance, mitochondrial DNA. Therefore a quality control of mitochondria is very important to maintain cellular homeostasis (Nieminen, 2003; Keeble and Gilmore, 2007). In addition to the oxidant defence system (Jamieson, 1998), the selective degradation of mitochondria via autophagy termed mitophagy might fulfil this indispensable role.

In this study, methods for the induction and detection of mitophagy should be established.

\subsubsection{Chromosomal integration of Om45-GFP}

To enable the detection of mitophagy with plasmid-encoded marker proteins, similar to the marker proteins GFP-Atg8 or Pgk1-GFP for the detection of unselective autophagy, Om45GFP and Idh1-GFP were constructed (see 3.3.15).

The use of these plasmids for degradation assays did not result in autophagic degradation depended on Atg1 and Pep4 (data not shown), therefore GFP was chromosomally integrated at the Om45 locus via homologous recombination in some strains of interest, as described (Kanki, 2009a) (see 3.3.13). These strains were used for degradation assays (see 3.4.5.3). The cells were grown up to 3 days in YPL medium, containing lactate as sole carbon source, samples were taken at 24, 48 and 72 hours, alkaline lysed and immunoblotted.

Wild type cells expressing Om45-GFP showed a weak GFP signal after 48 hours growth in YPL, which increased after 72 hours. In contrast, the negative controls atg $1 \Delta$ and pep $4 \Delta$ showed no free GFP, due to their defects in autophagic degradation or vacuolar breakdown, respectively. 


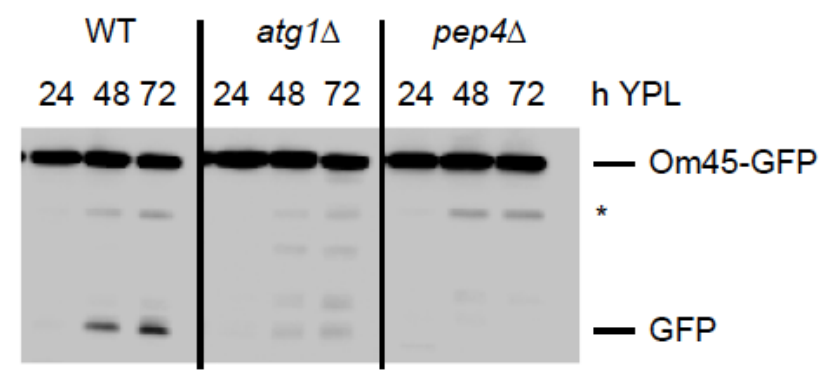

Figure 29: Monitoring mitophagy by western blot using Om45-GFP

Wild type (WT), atg $1 \Delta$ and pep $4 \Delta$ cells chromosomally expressing the mitochondrial Om45-GFP were grown up to 3 days in YPL medium. Samples were taken at 24, 48 and 72 hours, alkaline lysed and immunoblotted. Using monoclonal mouse anti-GFP antibody Om45-GFP ( 70 kDa) and GFP ( 26 $\mathrm{kDa})$ were detected. *unspecific crossreaction

\subsubsection{The pre-autophagosomal structure localises to mitochondria}

Up to now, it is still unclear if the mitochondrial cargo is transported to the PAS or if the PAS assembles at the mitochondria.

Wild type and atg1s cells chromosomally expressing Om45-GFP were used for fluorescence microscopy. The cells, additionally expressing the PAS marker RFP-Atg8, were observed in a DeltaVision microscope after starvation of 5 hours in SD-N medium.

Both strains, wild type and $\operatorname{atg} 1 \Delta$, showed clear red punctate structures, representing the PAS, in close proximity to mitochondria, indicating the specific recruitment of the autophagic machinery for initiation of mitophagy.

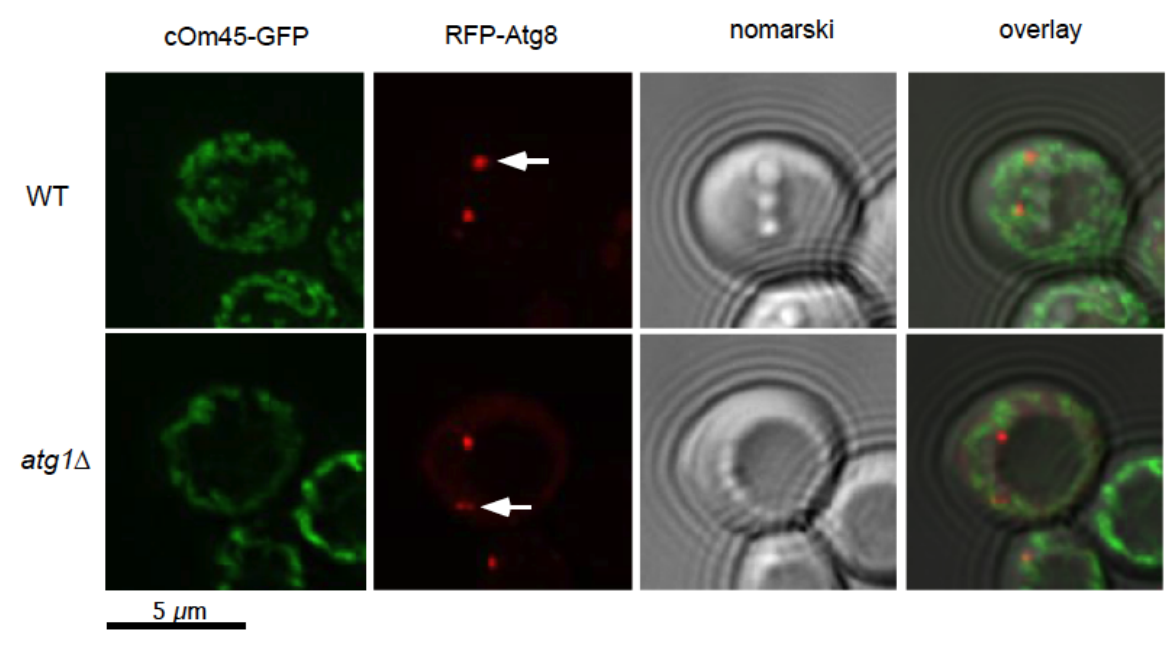

Figure 30: Localisation of the PAS at mitochondria

Wild type (WT) and atg1 $1 \Delta$ cells (WCG) chromosomally expressing Om45-GFP and RFP-Atg8 from plasmid were cultured in $\mathrm{CM}$-ura $+2 \%$ lactate for 3 days and visualised by fluorescence microscopy using a DeltaVision microscope equipped with FITC and TRITC filters. The white arrow indicates a PAS-like structure distant from the vacuole. 


\subsubsection{Construction of mito-GFP}

Chromosomal tagging of Om45 is laborious (Kanki, 2009a), therefore a plasmid based assay should be established. Su9-mt-DHFR was inserted into the vector pUG35 containing a GFP sequence under control of the MET25 promoter (mito-GFP) (see 3.2.10.2).

The mito-GFP plasmid was transformed in WCG wild type cells. These cells were grown to $\log$ phase in $\mathrm{CM}$-ura - met $+2 \%$ lactate and visualised using a DeltaVision microscope equipped with a FITC filter. Under these conditions mito-GFP showed green tubular structures representing mitochondria (Figure $31(\mathrm{~A})$ ).

Furthermore wild type, atg $1 \Delta$ and pep $4 \Delta$ cells transformed with mito-GFP were grown up to 3 days in $\mathrm{CM}-$ ura - met $+2 \%$ lactate medium, samples were taken at 24, 48 and 72 hours, alkaline lysed and immunoblotted.

After $48 \mathrm{~h}$ of growth, wild type cells showed a clear signal for free GFP, increasing after 72 hours of growth. In contrast, the negative controls atgl $1 \Delta$ and pep $4 \Delta$ showed no signal (Figure $31(\mathrm{~B}))$.

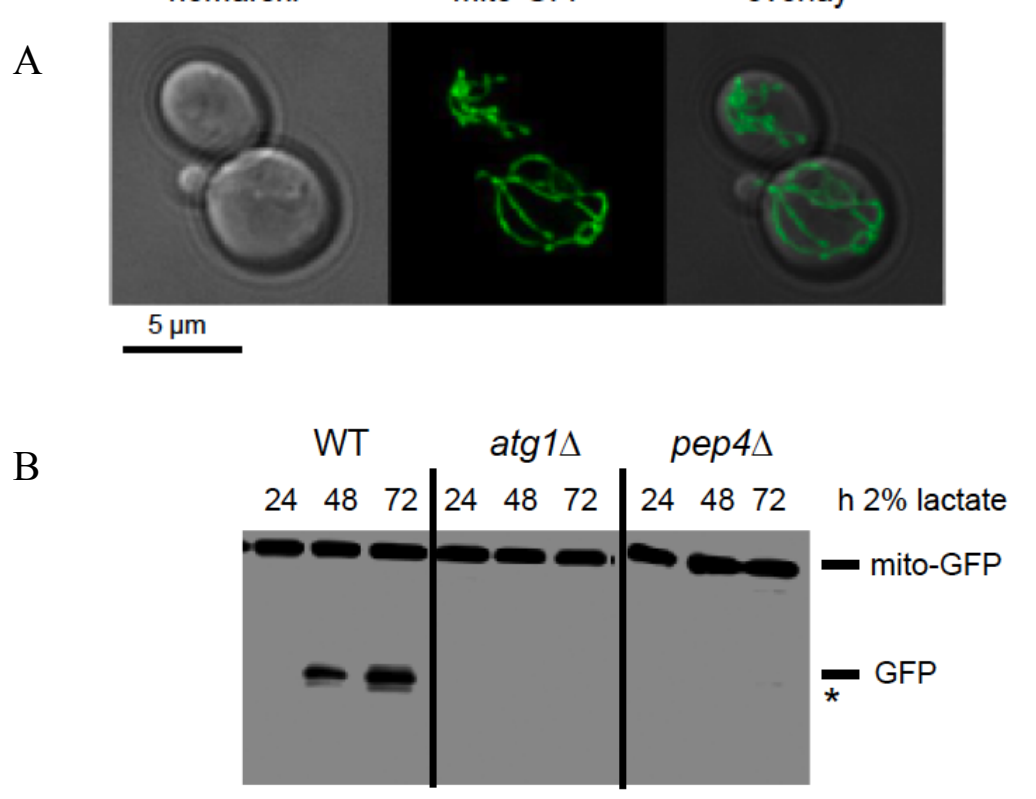

Figure 31: Fluorescence microscopy and western blot analysis of cells expressing mito-GFP

(A) Wild type (WCG) cells expressing mito-GFP were grown over night in CM-ura-met $+2 \%$ lactate and GFP was visualised by fluorescence microscopy using a DeltaVision microscope equipped with a FITC filter.

(B) Wild type (WT), atg $1 \Delta$ and pep $4 \Delta$ cells expressing mito-GFP were grown up to 3 days in CM-uramet $+2 \%$ lactate medium. Samples were taken 24,48 and 72 hours, alkaline lysed and immunoblotted. Using monoclonal mouse anti-GFP antibody mito-GFP $(\sim 48 \mathrm{kDa})$ and GFP $(\sim 26 \mathrm{kDa})$ were detected. *unspecific crossreaction 


\subsubsection{Different methods to induce mitophagy}

Kanki et al. (2009a) described two different conditions to induce mitophagy in S. cerevisiae cells grown on non-fermentable carbon source. One method is the induction by amino acid starvation, which means the shift of the cells grown to mid log phase from a nonfermentable carbon source to nitrogen starvation medium containing glucose. The other method is the above used induction at post-log phase, meaning a prolonged incubation for up to 3 days in lactate as sole carbon source. In recent publications these two conditions have led to some discrepancies concerning the requirement of different proteins for mitophagy (Kanki, 2009b; Okamoto, 2009). Aim of this study was the confirmation and establishment of different conditions to reliably induce mitophagy and to analyse the requirement of individual Atgproteins.

As control for variations in the general autophagic machinery caused by e.g. growth conditions, cells expressing Pgk1-GFP were included for visualisation of unselective autophagy (Welter, 2010). To ensure an adequate separation of the GFP signal and crossreactions, $10 \%$ SDS gels containing $6 \mathrm{M}$ urea were used for all experiments using mitoGFP. Post-log phase induced mitophagy by cultivation of the strains up to 3 days in lactate medium led to a clear signal of free GFP in wild type cells for both mitophagy (Figure 32 (A)) and unselective autophagy (Figure 32 (B)). No signal was observed in $\operatorname{atg} 18 \Delta$ cells, used as negative control. ATG13 deleted cells showed a block for unselective autophagy but a residual activity for mitophagy was be detected. The autophagic core protein Atg13, whose interaction with Atg1 is required for autophagy (Kamada, 2000), has already been discussed to be not essential for mitophagy (Okamoto, 2009). In agreement with literature, cells depleted in the mitophagy-specific receptor protein Atg32 showed no mitophagy, but significant unselective autophagy.

Another common method to induce autophagic processes, is the addition of rapamycin, the inhibitor of the TOR (target of rapamycin) kinase (Noda and Ohsumi, 1998). Mitophagy also depends on the autophagic core machinery including the TOR kinase (Kanki, 2009b; Okamoto, 2009). Hence, cells expressing mito-GFP or Pgk1-GFP were grown to mid $\log$ phase and treated with $0.2 \mu \mathrm{g} / \mathrm{ml}$ rapamycin. Samples were taken at 0, 2, 4 and 6 hours and treated as previously described. This method led to a clear signal for free GFP in wild type cells for both mitophagy (Figure 33 (A)) and unselective autophagy (Figure 33 (B)), but not in $\operatorname{atg} 18 \Delta$ cells. ATG13 deleted cells showed a block for unselective autophagy but a weak activity for mitophagy could be detected. The additional control atg32 showed no signal for mitophagy, in contrast to almost normal unselective autophagy. 

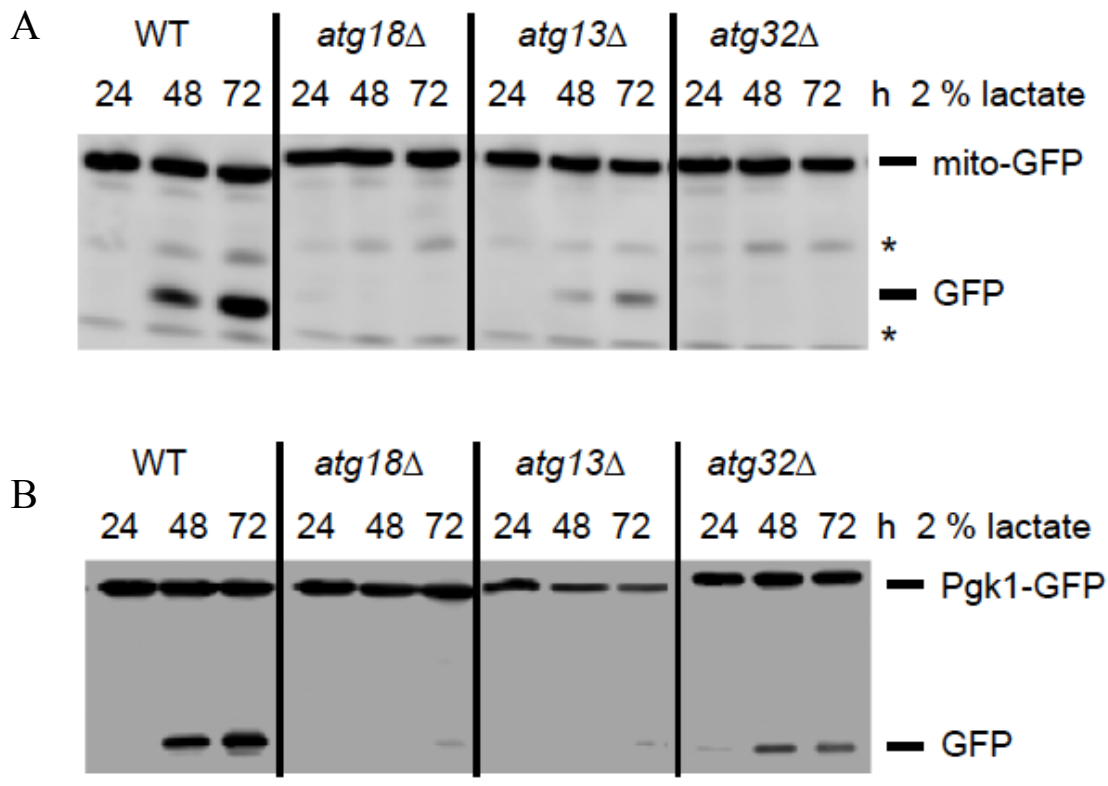

Figure 32: Incubation for 3 days in lactate medium induces mitophagy

Monitoring mitophagy (A) and unselective autophagy (B) by western blot using cells (BY4742)

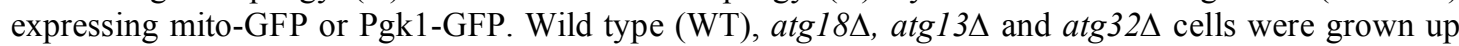
to 3 days in CM -ura -met $+2 \%$ lactate. Samples were taken at 24, 48 and 72 hours, alkaline lysed and immunoblotted. Using a monoclonal mouse anti-GFP antibody, mito-GFP ( $48 \mathrm{kDa})$, Pgk1-GFP ( 71 $\mathrm{kDa})$ and free GFP $(\sim 26 \mathrm{kDa})$ were detected. (A) $10 \%$ SDS gels containing $6 \mathrm{M}$ urea were used. *unspecific crossreaction

A

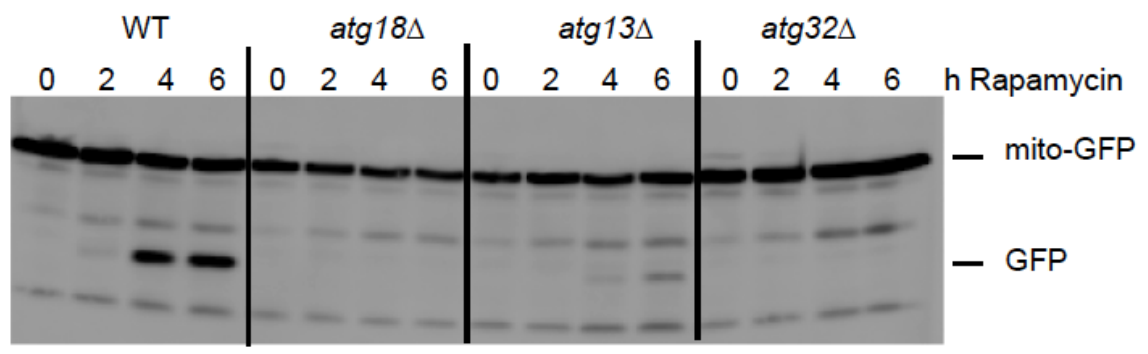

B

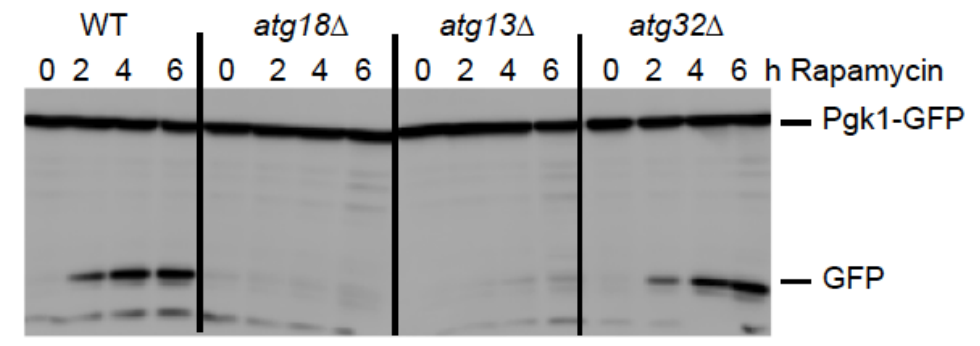

Figure 33: Rapamycin induces mitophagy

Monitoring mitophagy (A) and unselective autophagy (B) by western blot using cells (BY4742) expressing mito-GFP or Pgk1-GFP. Wild type (WT), $\operatorname{atg} 18 \Delta, \operatorname{atg} 13 \Delta$ and $\operatorname{atg} 32 \Delta$ were grown in lactate medium lacking methionine to mid-log growth phase and $0.2 \mu \mathrm{g} / \mathrm{ml}$ rapamycin was added. Sample were taken at $0,2,4$ and 6 hours, alkaline lysed and immunoblotted. Using a monoclonal mouse anti-GFP antibody, mito-GFP ( 48 kDa), Pgk1-GFP $(\sim 71 \mathrm{kDa})$ and free GFP $(\sim 26 \mathrm{kDa})$ were detected.

(A) 10 $\%$ SDS gels containing $6 \mathrm{M}$ urea were used. *unspecific crossreaction 
So far, using the described methods, superfluous mitochondria presented the cargo for mitophagy, but also damaged mitochondria might be a specific cargo for mitophagic degradation.

One of the major sources of reactive oxygen species (ROS) is hydrogen peroxide $\left(\mathrm{H}_{2} \mathrm{O}_{2}\right)$. Several studies demonstrated different roles for $\mathrm{H}_{2} \mathrm{O}_{2}$ in many biological processes, such as apoptosis and also autophagy (Kiffin, 2006; Chen, 2009). $\mathrm{H}_{2} \mathrm{O}_{2}$ is a product of incomplete one-electron reduction of oxygen, resulting in $\mathrm{O}_{2}{ }^{-}$which is converted to $\mathrm{H}_{2} \mathrm{O}_{2}$ by the superoxide dismutase (SOD) and furthermore altered by catalases to $\mathrm{H}_{2} \mathrm{O}$ (Chen, 2009).

In this thesis, $\mathrm{H}_{2} \mathrm{O}_{2}$ was tested as external stress factor and potential inducer of mitophagy by disturbing the intracellular conditions concerning the ROS balance.

Different concentrations of $\mathrm{H}_{2} \mathrm{O}_{2}$ were tested in wild type and atg18 $\Delta$ cells expressing mitoGFP (Figure 34 (A)). In parallel the survival rate was analysed, to avoid effects from dead cells (Figure34 (B)). As $0.05 \mathrm{mM} \mathrm{H}_{2} \mathrm{O}_{2}$ led to a measurable signal of free GFP in wild type cells but not in atg184 after 3 hours of treatment and a cell survival rate of $89 \%$, decreasing by the addition of $0.1 \mathrm{mM} \mathrm{H}_{2} \mathrm{O}_{2}$ to $83 \%$, this concentration was used for further experiments.

$0.05 \mathrm{mM} \mathrm{H}_{2} \mathrm{O}_{2}$ was added to cells grown to mid log phase, samples were taken at defined time points $(0,2,4$ and $6 \mathrm{~h})$ and treated as previously described. Figure 34 shows that treatment with $\mathrm{H}_{2} \mathrm{O}_{2}$ led to a clear signal for free GFP in wild type cells for both mitophagy (C) and unselective autophagy (D). ATG13 deleted cells showed a block for unselective autophagy but again a weak mitophagy activity was detected. The additional control $\operatorname{atg} 32 \Delta$ showed no signal for mitophagy, in contrast to almost normal unselective autophagy. 
A
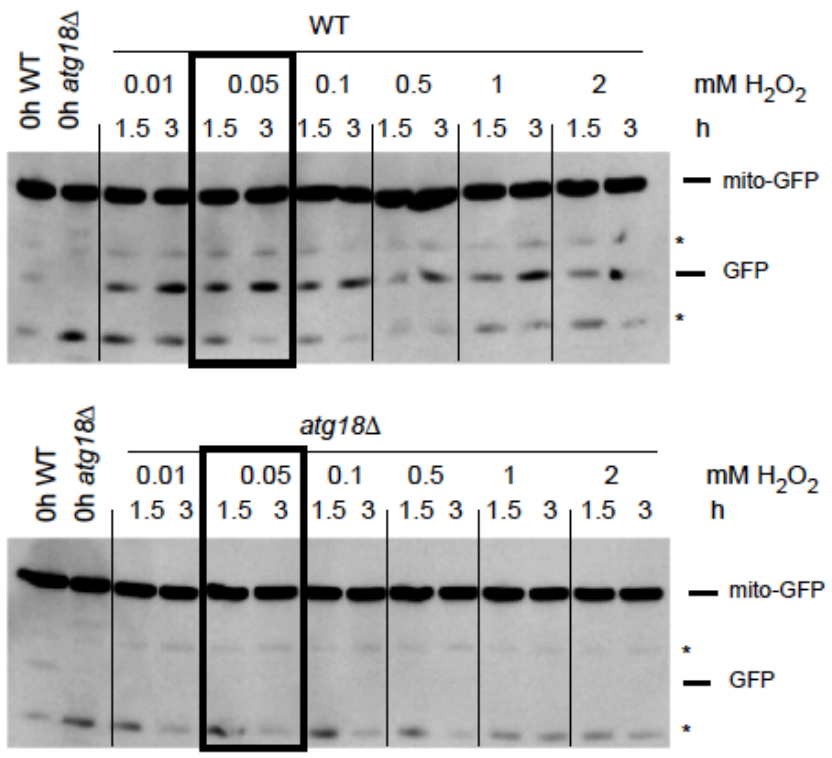

B

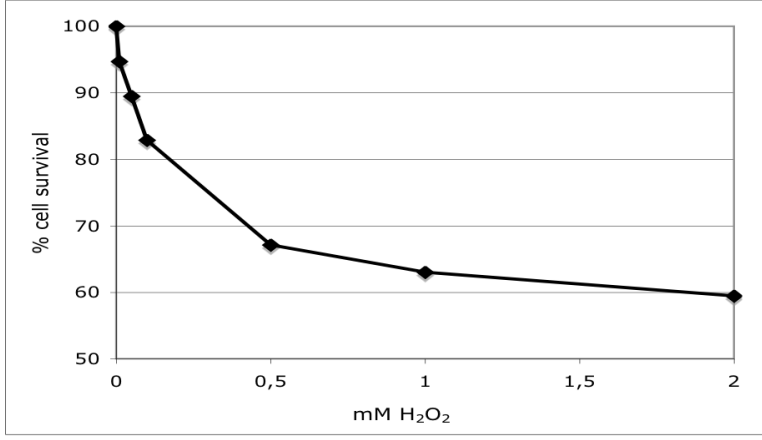

WT $\operatorname{atg} 18 \Delta \quad \operatorname{atg} 13 \Delta \quad \operatorname{atg} 32 \Delta$

C

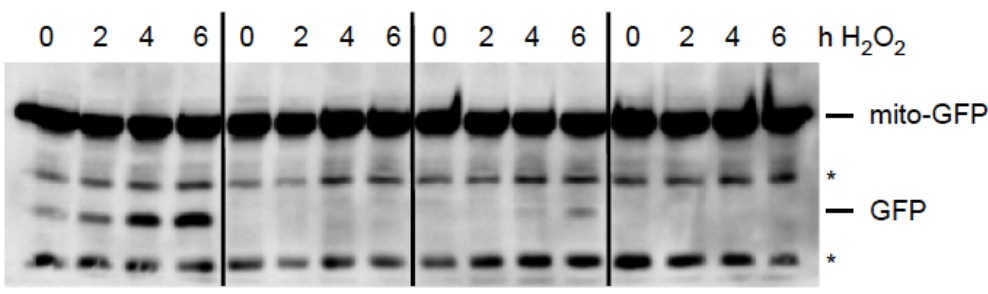

D

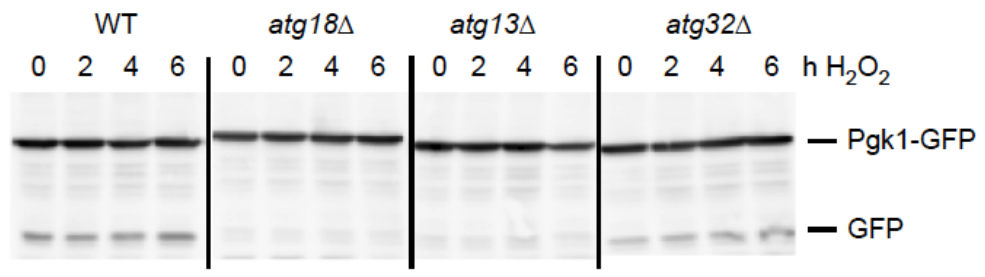

Figure 34: Mitophagy is induced by oxidative stress $\left(\mathrm{H}_{2} \mathrm{O}_{2}\right)$

(A) Different concentrations of $\mathrm{H}_{2} \mathrm{O}_{2}$ were tested in wild type and $\operatorname{atg} 18 \Delta$ cells (BY4742) expressing mito-GFP. (B) In parallel the survival rate was analysed, to exclude excessive cell death.

(C) and (D) Monitoring mitophagy (C) and unselective autophagy (D) by western blot using cells

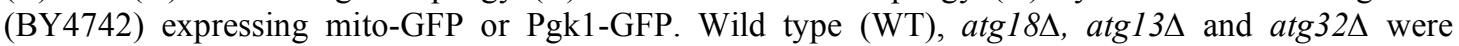
grown in lactate medium lacking methionine to mid-log growth phase and $0.05 \mathrm{mM} \mathrm{H}_{2} \mathrm{O}_{2}$ was added. Samples were taken at 0, 2, 4 and 6 hours, alkaline lysed and immunoblotted. Using a monoclonal mouse anti-GFP antibody, mito-GFP $(\sim 48 \mathrm{kDa})$, Pgk1-GFP $(\sim 71 \mathrm{kDa})$ and free GFP $(\sim 26 \mathrm{kDa})$ were detected. (A) and (C) $10 \%$ SDS gels containing $6 \mathrm{M}$ urea were used. *unspecific crossreaction 
Another compound causing oxidative stress is N, N'-dimethyl-4,4'-bipyridinium dichloride (paraquat). It catalyses the formation of ROS by the acceptance of electrons from an electron donor (e.g. NADPH) and the further oxidation of an electron receptor such as dioxygen. Paraquat therefore mainly produces superoxide anions $\left(\mathrm{O}_{2}^{-}\right)$and $\mathrm{H}_{2} \mathrm{O}_{2}$, yielding in the impairment of the mitochondrial function.

To test if paraquat induces mitophagy, wild type and atg18 cells expressing mito-GFP were treated with different concentrations of paraquat up to 6 hours (Figure 35 (A)). In parallel the survival rate was analysed, to exclude excessive cell death (Figure 35 (B)). As $2 \mathrm{mM}$ paraquat led to a measurable signal for free GFP in western blot experiments and a cell survival rate of $84 \%$, decreasing by the addition of $5 \mathrm{mM}$ paraquat to $81 \%$, this concentration was used for further experiments.

Wild type, $\operatorname{atg} 18 \Delta, \operatorname{atg} 13 \Delta$ and $\operatorname{atg} 32 \Delta$ cells were grown to mid log phase and treated with $2 \mathrm{mM}$ paraquat. Samples were taken at 0,2, 4 and 6 hours and treated as described previously. Figure 35 demonstrates that the addition of paraquat led to a clear signal for free GFP in wild type cells using mito-GFP (C). A very weak signal could be detected for unselective autophagy (D). ATG13 deleted cells showed a block for unselective autophagy, but as above a very weak residual mitophagy activity was detected. The additional control $\operatorname{atg} 32 \Delta$ showed no mitophagy but some unselective autophagy. 


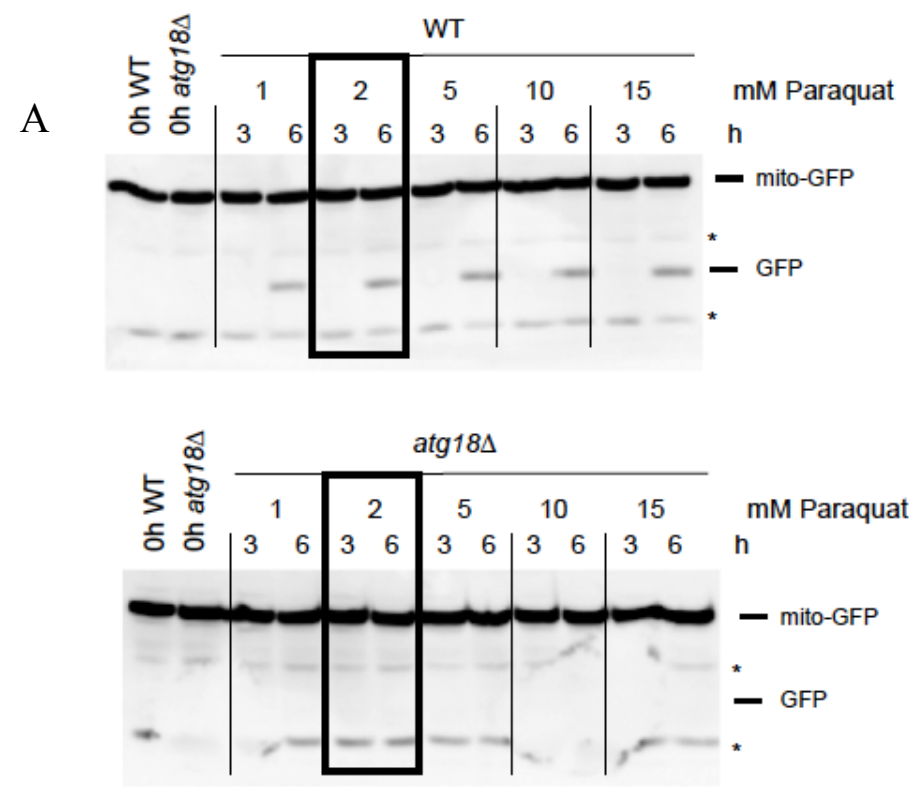

B

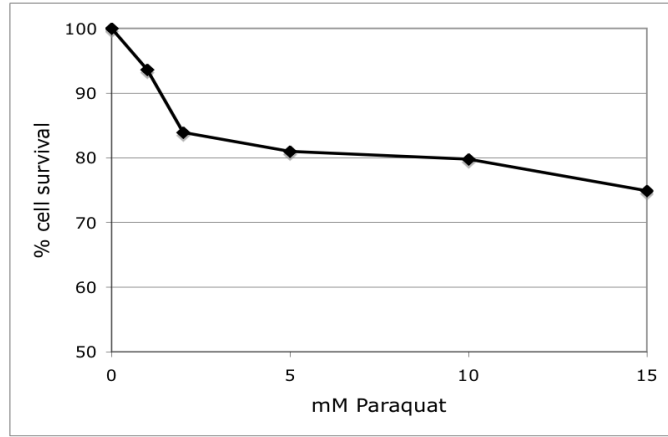

WT $\operatorname{atg} 18 \Delta \quad \operatorname{atg} 13 \Delta \quad \operatorname{atg} 32 \Delta$

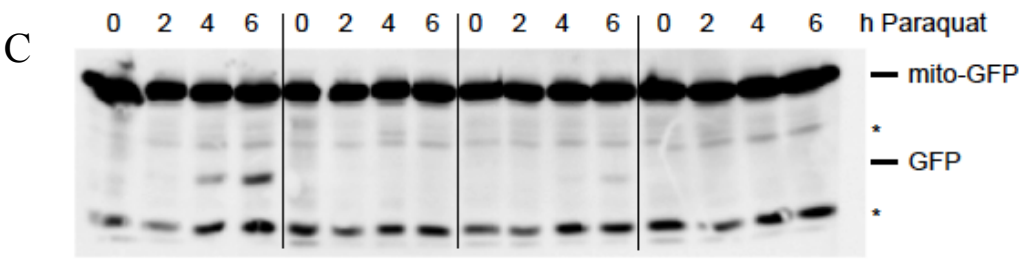

WT $\operatorname{atg} 18 \Delta \quad \operatorname{atg} 13 \Delta \quad \operatorname{atg} 32 \Delta$

$\mathrm{D}$

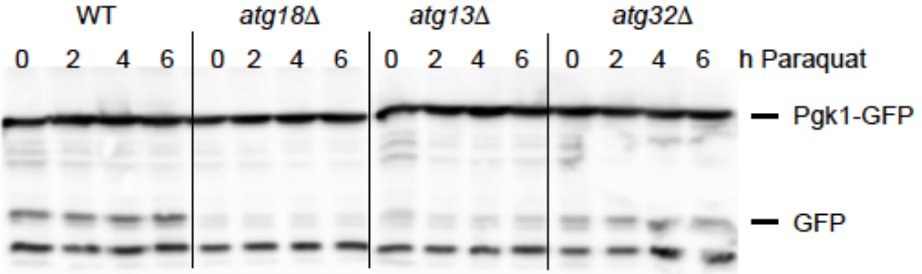

Figure 35: Paraquat induces mitophagy

(A) Different concentrations of paraquat were tested in wild type and atg184 cells (BY4742) expressing mito-GFP. (B) In parallel the survival rate was analysed, to exclude excessive cell death.

(C) and (D) Monitoring mitophagy (A) and unselective autophagy (B) by western blot using cells (BY4742) expressing mito-GFP or Pgk1-GFP. Wild type (WT), $\operatorname{atg} 18 \Delta, \operatorname{atg} 13 \Delta$ and $\operatorname{atg} 32 \Delta$ were cultured in lactate medium lacking methionine to mid-log growth phase and $2 \mathrm{mM}$ paraquat was added. Samples were taken at 0,2, 4 and 6 hours, alkaline lysed and immunoblotted. Using a monoclonal mouse anti-GFP antibody, mito-GFP $(\sim 48 \mathrm{kDa})$, Pgk1-GFP $(\sim 71 \mathrm{kDa})$ and free GFP $(\sim 26 \mathrm{kDa})$ were detected. (A) and (C) $10 \%$ SDS gels containing $6 \mathrm{M}$ urea were used. *unspecific crossreaction 


\subsubsection{Mitophagy is increased in yme $1 \Delta$ cells}

Ymel is a subunit of the mitochondrial inner membrane i-AAA protease complex. It is responsible for the degradation of unfolded or misfolded mitochondrial proteins and therefore involved in aging and apoptotic response (Leonhard, 1996; Palermo, 2007). The accumulation of dysfunctional mitochondria in ymels cells might therefore induce mitophagy (Campbell and Thorsness, 1998). To test if the mitophagic rate is increased in

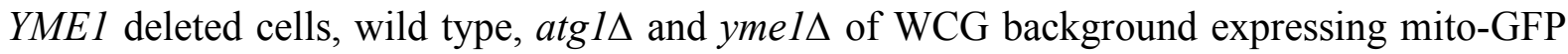
were grown for up to 3 days in $\mathrm{CM}$-ura -met medium containing 2\% lactate. Samples were taken at 24, 48 and 72 hours, alkaline lysed and used for western blots. For evaluation of mitophagy, signal intensities of mito-GFP and free GFP were quantified. The ratio of free GFP to mito-GFP was calculated. The value of wild type cells after 72 hours of growth was set to $100 \%$. Three independent experiments were done and the resulting values were illustrated (Figure 36). Since atg $1 \Delta$ cells are defective in both, unselective and selective autophagy, no signal was detected in this negative control. In contrast, yme1 $\Delta$ cells showed an increased rate of mitophagy of about $57 \%$ after 24 hours, $113 \%$ after 48 hours and $122 \%$ after 72 hours of growth compared to wild type cells. After 24 hours under non starvation conditions, the mitophagy rate is already increased by 3.5 times in ymel $\Delta$ cells compared to wild type cells (14\%), indicating the degradation of damaged and not superfluous mitochondria.

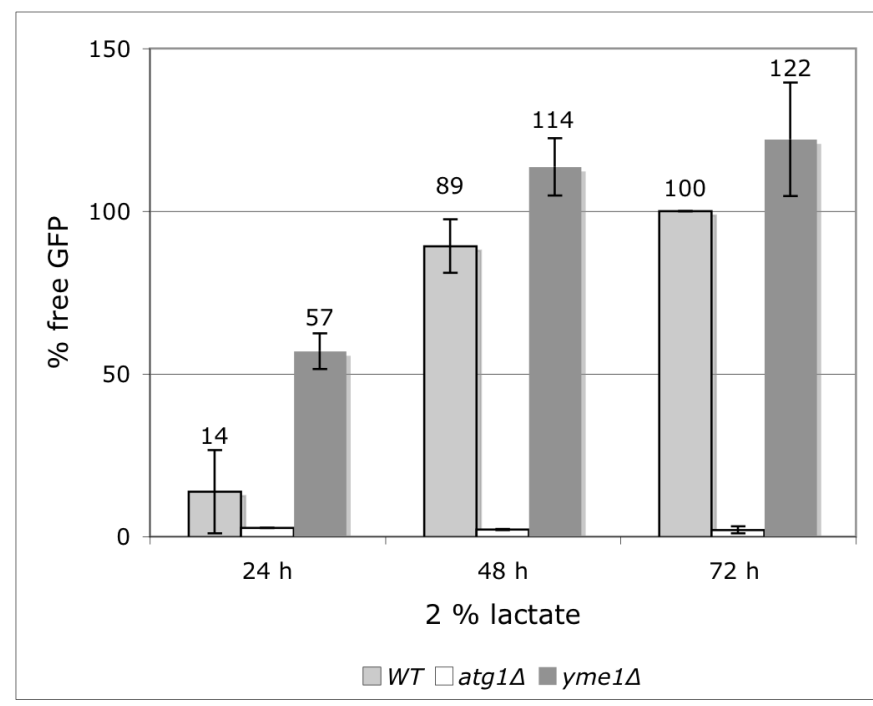

Figure 36: Quantification of mitophagy in yme1 $\Delta$

Wild type (WT), atg $1 \Delta$ and yme $1 \Delta$ (WCG background) were grown in CM -ura - met $+2 \%$ lactate for 72 hours. Samples were taken at 24, 48 and 72 hours, alkaline lysed and immunoblotted. Using a monoclonal mouse anti-GFP antibody, mito-GFP $(\sim 48 \mathrm{kDa})$ and free GFP $(\sim 26 \mathrm{kDa})$ were detected and quantified with AIDA software. The ratio of free GFP to mito-GFP was calculated and the value of wild type cells after 72 hours of growth was set to $100 \%(n=3)$. 


\subsubsection{Part of the ESCRT machinery is required for mitophagy}

As the involvement of the ESCRT machinery in PMN is discussed (Bremer, 2009), exemplary members (Table 19) were examined for their involvement in mitophagy compared to unselective autophagy in this study. Different strains, lacking one ESCRT protein and additional control strains were transformed with mito-GFP and used in the mitophagy assay, parallel to control analysis with Pgk1-GFP.

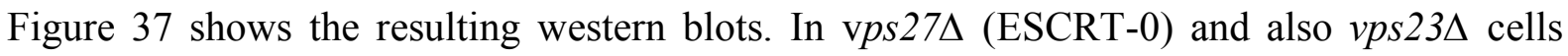
(ESCRT-I) a high signal intensity of free GFP for mitophagy and for unselective autophagy similar to wild type cells was seen. The negative control atgls exhibited no signal (Figure 37(A)). In contrast, vps $28 \Delta$ cells showed a clear reduction in free GFP for mitophagy but not for unselective autophagy (Figure 37 (B)). Therefore further ESCRT members were tested.

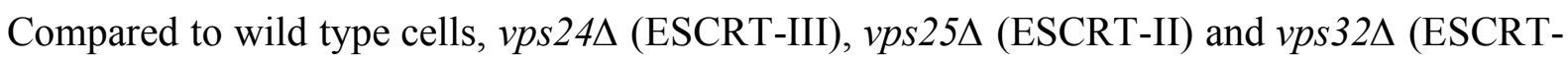
III) showed a clear signal reduction of free GFP for mitophagy but not of unselective autophagy. In atg18 cells a complete block for both pathways was seen (Figure 37 (C)). Up to four independent experiments were made. The western blots were quantified using AIDA software. The calculated signal intensity of free GFP in ratio to mito-GFP or Pgk1-GFP in wild type cells after 3 days of growth was set to $100 \%$. Figure 37 (D) summarises the role of some ESCRT subunits in mitophagy. The quantification supports the findings that the deletion of VPS23 and VPS27 does not affect mitophagy. In contrast, the deletion of VPS24, VPS25, VPS28 and VPS32 seemed to impair mitophagy. Table 19 summarises the tested ESCRT deletion strains and their requirement for unselective autophagy or mitophagy and highlights the involvement of the ESCRT subunits II and III in mitophagy, while the ESCRT subunits 0 and I are not involved. 
A
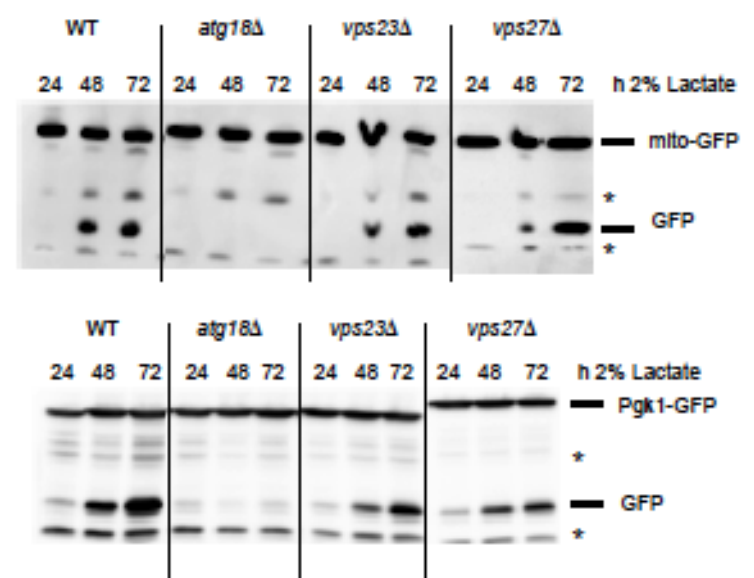

B

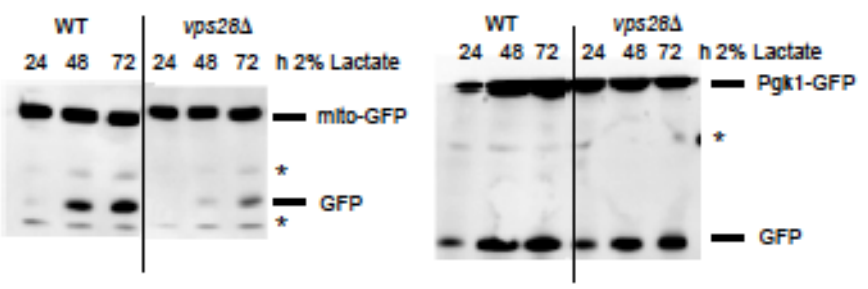

$\mathrm{C}$
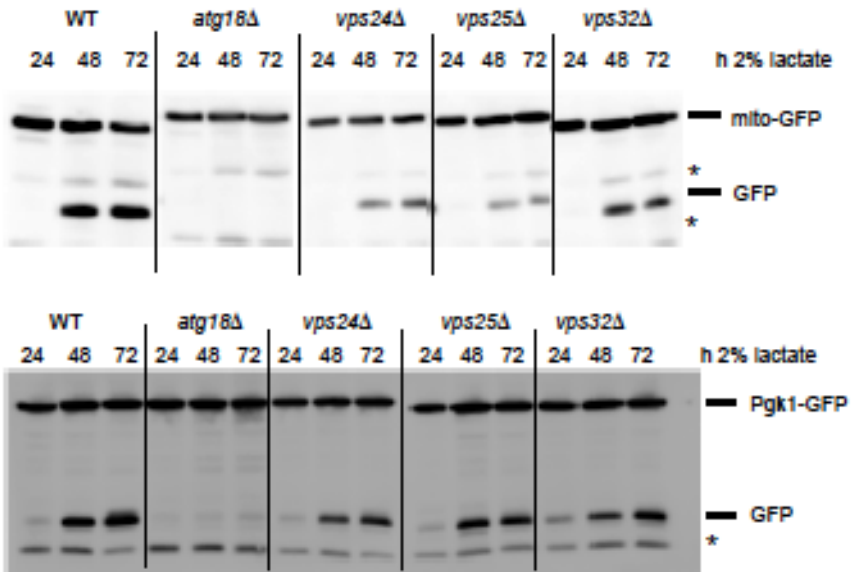

$\mathrm{D}$

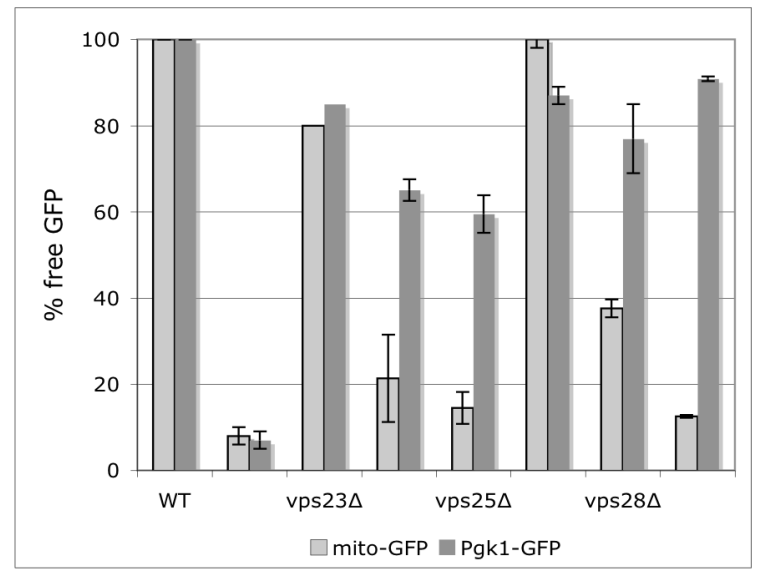

Figure 37: Part of the ESCRT machinery is required for mitophagy

(A-C) Wild type cells, atg18 and different strains deleted in one member of the ESCRT machinery (BY4742) expressing mito-GFP or Pgk1-GFP were grown up to 3 days in CM-ura -met $+2 \%$ lactate, samples were taken at 24, 48 and 72 hours, alkaline lysed and immunoblotted. Using a monoclonal mouse anti-GFP antibody, mito-GFP ( $48 \mathrm{kDa})$, Pgk1-GFP $(\sim 71 \mathrm{kDa})$ and free GFP $(\sim 26 \mathrm{kDa})$ were detected. $10 \%$ SDS gels containing $6 \mathrm{M}$ urea were used. *unspecific crossreaction

(D) The western blots were quantified using AIDA software. The calculated signal intensity of free GFP in ratio to mito-GFP or Pgk1-GFP in wild type cells after 3 days of growth was set to $100 \%$ $(1 \leq \mathrm{n} \geq 4)$. 
Table 19: Summary of tested ESCRT mutants

All tested ESCRT deletion mutants are listed including quantification values of unselective autophagy and mitophagy

\begin{tabular}{|c|c|c|c|}
\hline strain & & autophagy & mitophagy \\
\hline $\operatorname{atg} 18 \Delta$ & & $7 \%$ & $8 \%$ \\
\hline$v p s 23 \Delta$ & ESCRT-I & $85 \%$ & $80 \%$ \\
\hline$v p s 24 \Delta$ & ESCRT-III & $65 \%$ & $21 \%$ \\
\hline$v p s 25 \Delta$ & ESCRT-II & $60 \%$ & $15 \%$ \\
\hline$v p s 27 \Delta$ & ESCRT-O & $87 \%$ & $100 \%$ \\
\hline$v p s 28 \Delta$ & ESCRT-I & $77 \%$ & $38 \%$ \\
\hline$v p s 32 \Delta$ & ESCRT-III & $91 \%$ & $13 \%$ \\
\hline
\end{tabular}

\subsubsection{Doa4 is involved in mitophagy but not in unselective autophagy}

Doa4, an ubiquitin isopeptidase, is required for recycling of ubiquitin from proteasome-bound polyubiquitinated proteins. The deletion of Doa4 depletes the cellular ubiquitin level (Swaminathan, 1999; Ren, 2008). In mammalian cells, ubiquitin plays an irreplaceable role in designating mitochondria for mitophagy (Matsuda, 2010). Probably, a decrease of the cellular ubiquitin level also affects mitophagy in S. cerevisiae. Therefore a role of Doa4 in mitophagy was tested. In addition to the control cells wild type, $\operatorname{atg} 18 \Delta$ and $\operatorname{atg} 32 \Delta$, doa $4 \Delta$ cells were transformed with mito-GFP and used for the mitophagy assay, parallel to the same strains expressing Pgk1-GFP. Figure 38 (A) shows a clear reduction of the signal for free GFP for mitophagy in doa $4 \Delta$ cells compared to wild type cells.

Quantification was done by AIDA software and the calculated signal intensity of free GFP in ratio to mito-GFP or Pgk1-GFP in wild type cells after 3 days of growth was set to $100 \%$ (Figure 38 (B)). The two negative controls atg184, blocked in all autophagic pathways, and atg32 , selectively blocked for mitophagy (Kanki and Klionsky, 2009; Okamoto, 2009), showed no free GFP signal for mitophagy. In doa4t cells the signal of free GFP appeared strongly reduced for mitophagy. Unselective autophagy was only reduced in doa $4 \Delta$ cells. Wild type and atg32 2 cells showed normal autophagy rates. Six independent experiments were done. The quantification of these western blots, which was done for exact evaluation of the signal for free GFP (Figure 38 (B)), confirmed the slight reduction of the GFP-signal for unselective autophagy in doa $4 \Delta$ cells $(65 \%$; SEM $=11)$ compared to wild type cells and the more significant reduction for mitophagy $(37 \%$;EM $=11)$. 
A

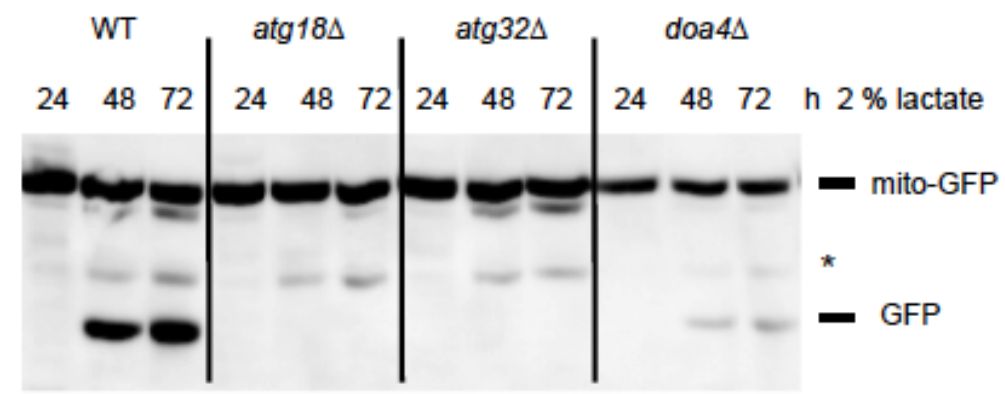

WT

$\operatorname{atg} 18 \Delta \quad \operatorname{atg} 32 \Delta \quad$ doa4 $\Delta$
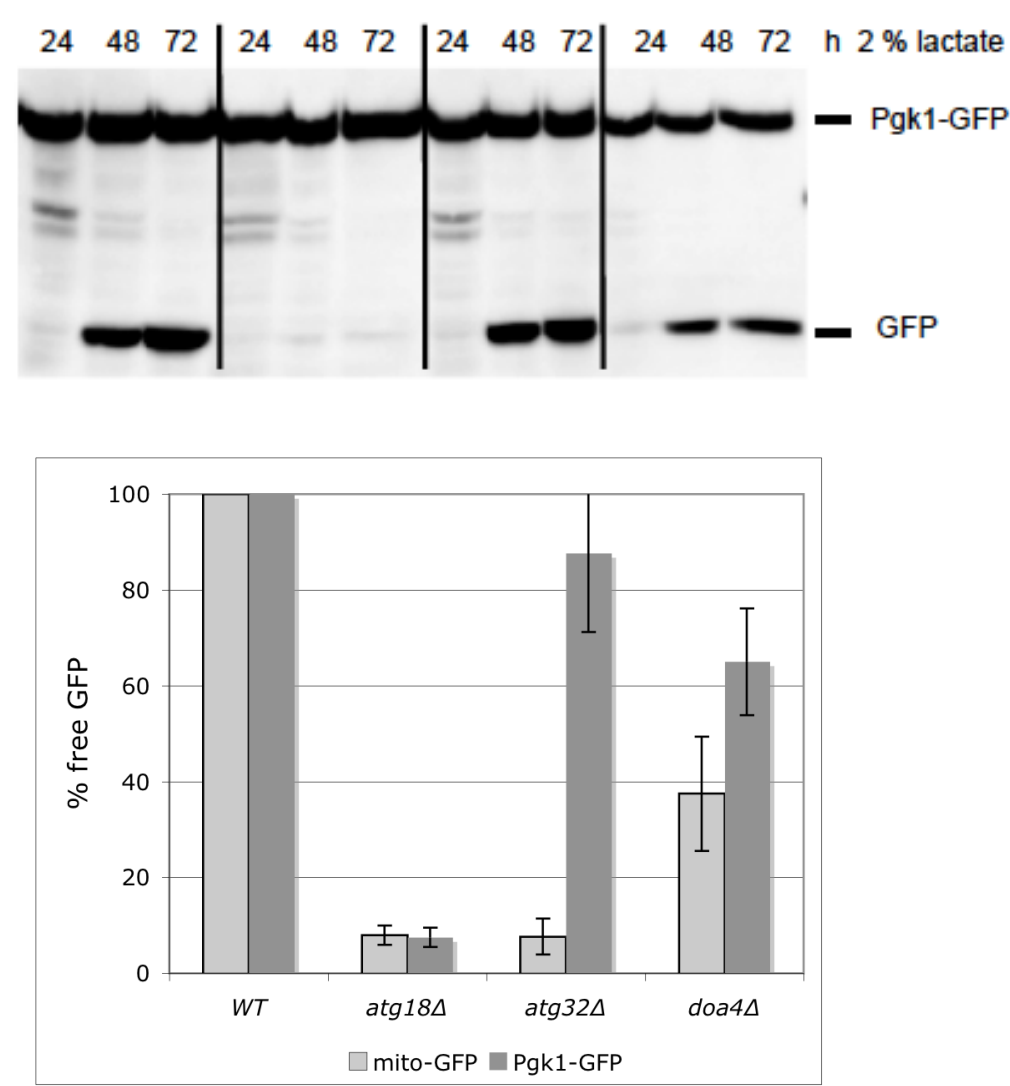

Figure 38: Doa4 is needed for mitophagy

(A) Monitoring mitophagy and unselective autophagy by western blot using cells expressing mito-GFP

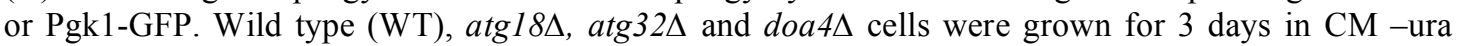
- met $+2 \%$ lactate. Samples were taken at 24, 48 and 72 hours, alkaline lysed and immunoblotted. Using a monoclonal mouse anti-GFP antibody, mito-GFP ( 48 kDa), Pgk1-GFP ( 71 kDa) and free GFP $(\sim 26 \mathrm{kDa})$ were detected. $10 \%$ SDS gels containing $6 \mathrm{M}$ urea were used. *unspecific crossreaction (B) GFP signals were quantified using AIDA software and the ratio of free GFP to mito-GFP or Pgk1GFP in wild type cells after 3 days of growth was set to $100 \%(n=6)$. 


\subsection{Piecemeal microautophagy of the nucleus}

Piecemeal microautophagy of the nucleus PMN is the selective microautophagic degradation of parts of the nucleus, which requires a subset of autophagic proteins (Roberts, 2003; Krick, 2008b).

Osh1, a member of the OSH family in yeast and a homologue of mammalian oxysterolbinding protein (OSBP) (Levine and Munro, 2001), may function in nonvesicular lipid trafficking. Osh1 is targeted to nucleus-vacuole (NV) junctions through physical interaction with Nvj1. Due to the interaction with NV junctions, Osh1 exhibits a substrate for PMN and is therefore a commonly used marker protein (Millen, 2009).

Besides the PMN dependent degradation of proteins involved in the formation of $\mathrm{NV}$ junctions (Roberts, 2003; Kvam, 2005), at the current state of research, a specific nuclear cargo for PMN is still unknown and is a major focus of investigation.

\subsubsection{Screen for selective PMN cargos}

In this thesis, 79 strains expressing different GFP fusion proteins (Table 5; Invitrogen) were tested for their autophagic degradation by the detection of free GFP in western blot analysis (similar to 3.4.5). Aim of this experiment was to find a specific PMN cargo. The set of the chosen GFP fusion proteins mainly included proteins of the nucleus and the nucleolus and further included proteins of other organelles such as mitochondria and the ER as controls. Exemplary for all strains used in this experiments, nucleolar located proteins are shown (Figure 39).

All strains were starved in SD-N up to 6 hours and samples were taken at 0 and 6 hours. The cells were alkaline lysed and used for western blot analysis. Chromosomally expressed Pgk1GFP was used as control for the induction of unselective autophagy and showed normal amounts of free GFP. Surprisingly, no degradation of any of the nucleolar proteins was detected. 


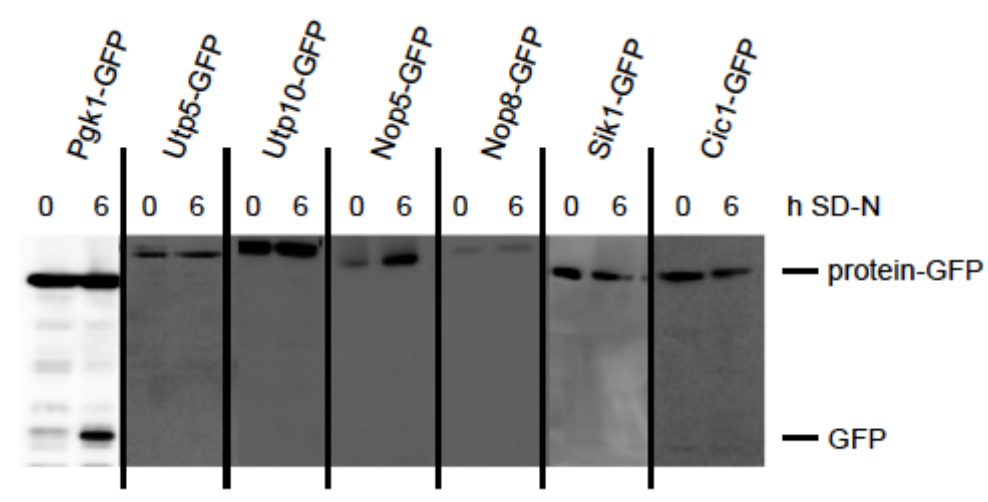

Figure 39: Western Blot analysis for the identification of specific PMN cargos

Different strains expressing nucleolus-located proteins with a GFP-tag and a strain chromosomally expressing Pgk1-GFP were starved for 6 hours. Samples were taken after 0 and $6 \mathrm{~h}$, alkaline lysed and immunoblotted. Using a monoclonal mouse anti-GFP antibody, GFP signals (Pgk1-GFP ( 71 kDa), Utp5-GFP ( 98 kDa), Utp10-GFP ( 226 kDa), Nop5-GFP ( 82 kDa), Nop8-GFP ( 82 kDa), Sik1-GFP $(\sim 83 \mathrm{kDa}), \mathrm{Cic1-GFP}(\sim 69 \mathrm{kDa})$ and free GFP $(\sim 26 \mathrm{kDa}))$ were detected.

\subsubsection{Microscopic analysis of nucleolar GFP fusion proteins}

Different GFP-tagged nucleolus proteins (Invitrogen) were used for microscopic analysis. The cells were incubated with FM4-64 for staining of the vacuolar membrane (see 3.2.10.2.2), starved in SD-N medium containing $1 \mathrm{mM}$ PMSF up to 4 hours and visualised using a DeltaVision microscope (FITC, TRITC) (Figure 40).

In contrast to Utp5-GFP and Utp10-GFP, which showed no green signal outside of the nucleolus, additional GFP signals inside the vacuole were detected in Nop8-GFP cells. Sik1GFP expressing cells presented a conspicuous contact of Sik1-GFP to the vacuolar membrane, while in Cic1-GFP expressing cells, some small green fluorescent vesicles released into the vacuole were observed (Figure 40).

Nog1, a nuclear GTP-binding protein, is tethered in a complex to the nucleolus upon nutrient starvation or rapamycin treatment (Honma, 2006). Therefore cells chromosomally expressing Nog1-GFP (Invitrogen) were also used for microscopy.

The cells were incubated with FM4-64 and starved for 4 hours in SD-N containing $1 \mathrm{mM}$ PMSF. Using the DeltaVision microscope equipped with FITC and TRITC filter system, PMN structures in Nog1-GFP cells were monitored. PMN-like structures, formed like green noses, budding from the nucleolus into the vacuole were observed in about $30 \%$ of the cells (Figure 41). 


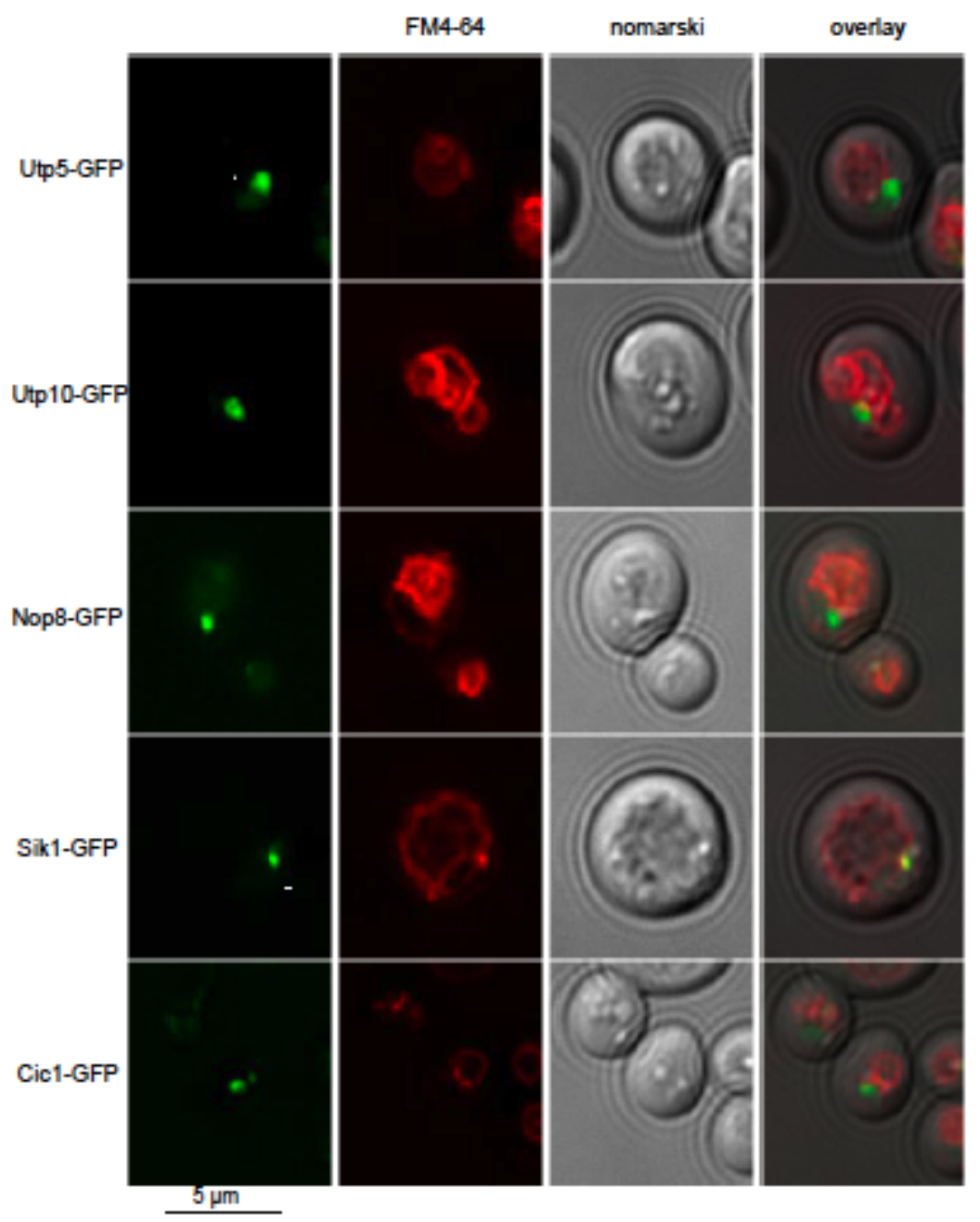

Figure 40: Microscopic analysis of strains expressing nucleolar GFP fusion proteins

Strains chromosomally expressing different nucleolar GFP fusion proteins (Invitrogen) were starved for 4 hours in SD-N medium containing 1 mM PMSF. The vacuoles were stained with FM4-64 (red).

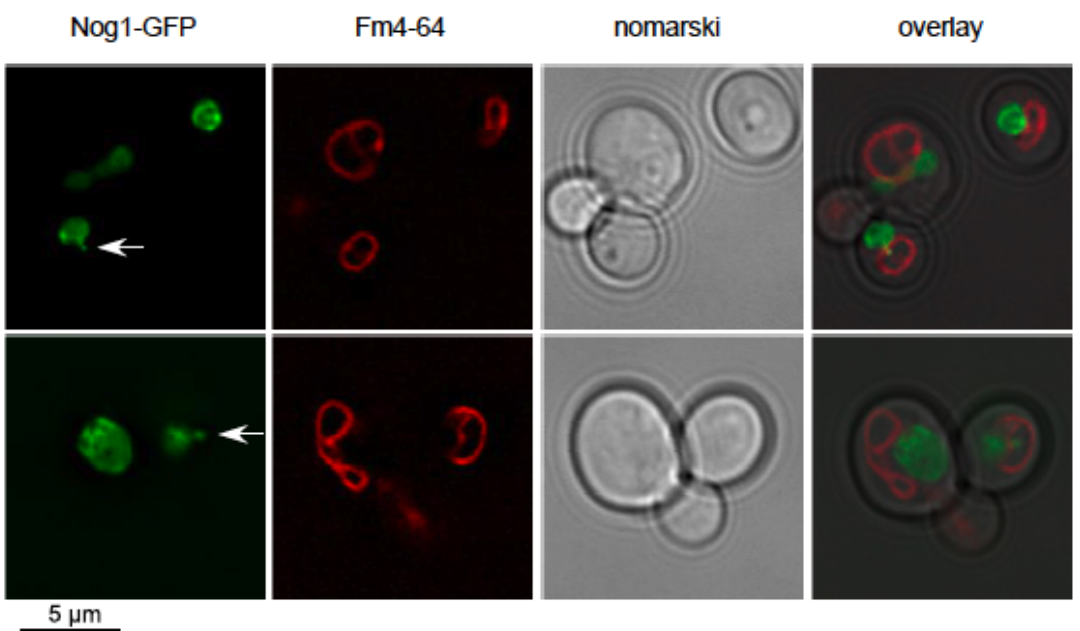

Figure 41: PMN structures in Nog1-GFP

Cells chromosomally expressing Nog1-GFP (Invitrogen) were starved for 4 hours in SD-N medium containing $1 \mathrm{mM}$ PMSF. The vacuoles were stained with FM4-64 (red). Clear green PMN-like structures pinching off the vacuolar membrane are marked with a white arrow. 


\subsubsection{Nucleus-vacuole junctions localise at the nucleolus}

Up on initiation of PMN, the cells form nucleus-vacuole junctions. These junctions can be visualised by GFP-tagged Osh1, which is targeted to NV junction through a physical interaction with Nvj1 (Kvam and Goldfarb, 2004). Sik1 (Nop56) is involved in rRNA processing and is located inside the nucleolus (Dragon, 2002; Bogomolnaya, 2004). Therefore cells chromosomally expressing Sik1-RFP were used for visualisation of the nucleolus in microscopy. To detect NV junctions and determine their localisation, these cells were transformed with Osh1-GFP.

The cells were starved for 4 hours in SD-N and visualised by using the DeltaVision microscope equipped with a FITC and TRITC filter system. Figure 42 (A) demonstrates the localisation of the NV junctions at the nucleolar part of the nucleus in wild type and atg $1 \Delta$ cells. In the negative control vac $8 \Delta$, no NV junctions were observed.

Counting more than 350 wild type cells in 5 independent experiments allowed a statistic evaluation. $70.6 \%(\mathrm{SEM}=0.6 \%)$ of $\mathrm{NV}$ junctions localised at the nucleolar part of the nucleus (Figure 42 (B)). These results indicated a specific uptake of nucleolar material by PMN. 
A

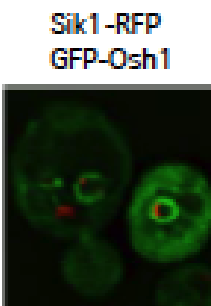

nomarski

overlay
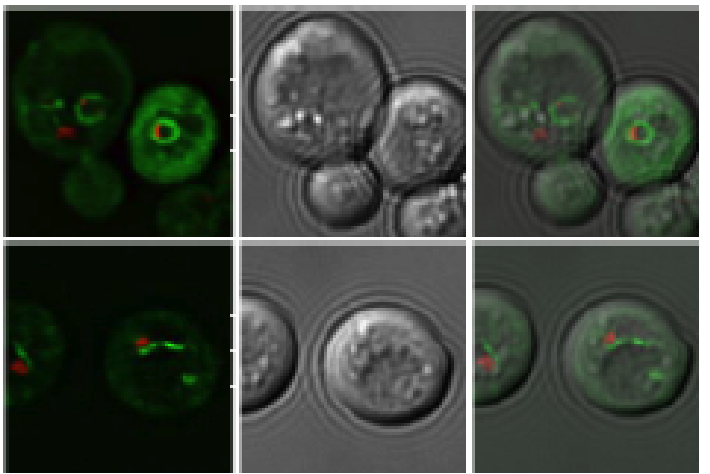

WT
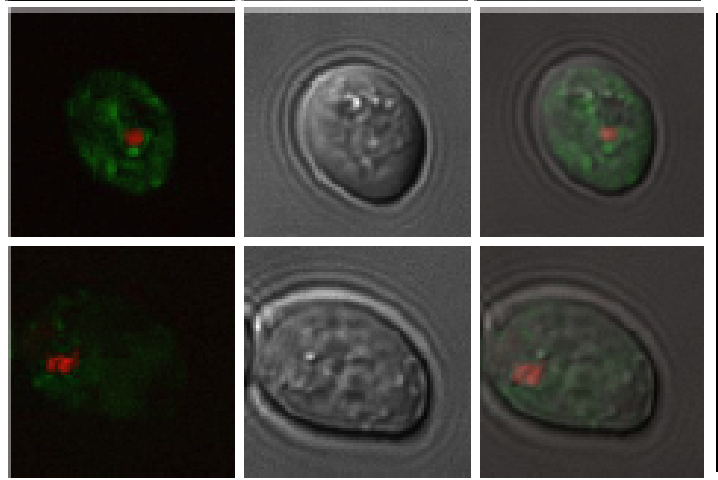

vac8s
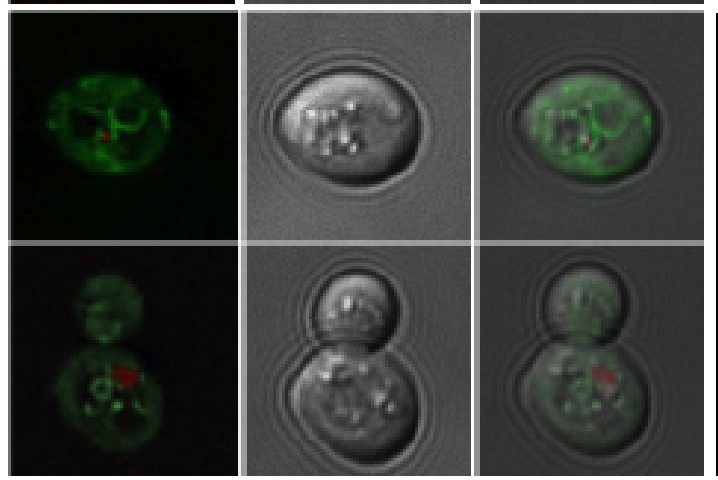

$5 . \mathrm{m}$

B

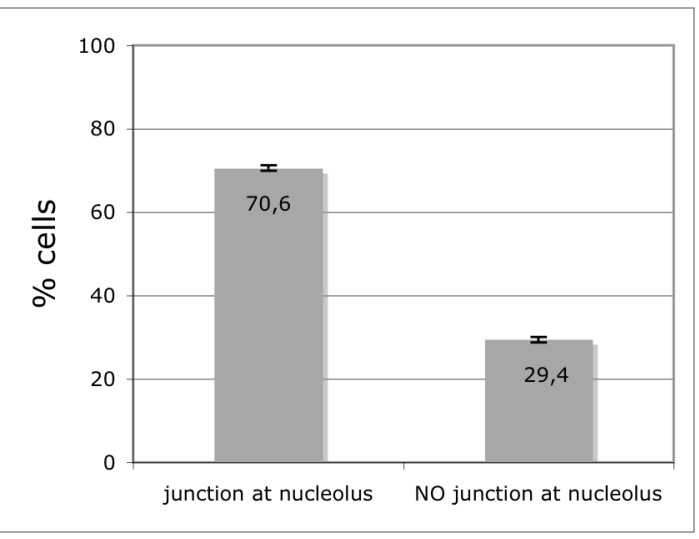

Figure 42: Nucleus-vacuole junctions localise adjacent the nucleolus

(A) Sik1-RFP cells containing Osh1-GFP were examined with the DeltaVision microscope (using FITC and TRITC filter). Wild type and atgl $1 \Delta$ cells chromosomally expressing Sik1-RFP showed clear green nucleus-vacuole junctions, while in $v a c 8 \Delta$ these junctions are absent.

(B) NV junctions were counted in 350 wild type cells in 5 independent experiments. $70.6 \%$ of NV junctions localised at the nucleolus $(\mathrm{SEM}=0.6 ; \mathrm{n}=5)$. 


\subsubsection{The PAS localises at the nucleolar part of the nucleus}

For the detection of the pre-autophagosomal structure (PAS), cells chromosomally expressing the nucleolar Sik1-RFP were transformed with GFP-Atg8. The cells were starved for 4 hours in SD-N medium. The visualisation of these cells using a DeltaVision microscope equipped with a FITC and TRITC filter system, presented a localisation of several PAS dots in close proximity to the nucleolus (Figure 43 (A)). Counting more than 100 cells in two independent experiments allowed a statistic evaluation resulting in $22.9 \%(\mathrm{SEM}=7.5 \%)$ cells exhibiting PAS dots in close proximity to the nucleolus (Figure 43 (B)).

A

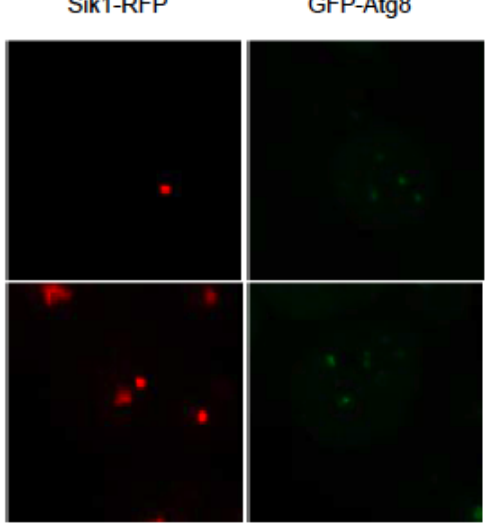

nomarski

overlay

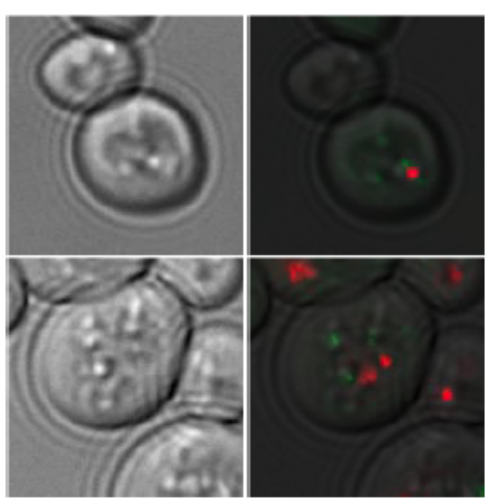

$5 \mu \mathrm{m}$

B

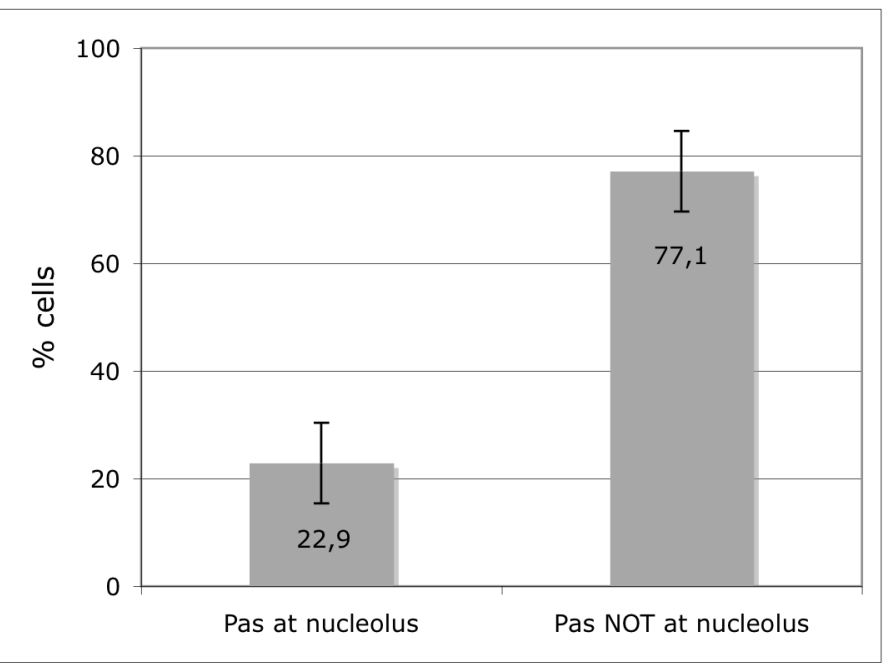

Figure 43: The pre-autophagosomal structure localises partially at the nucleolus

(A) Sik1-RFP (representing the nucleolus) cells expressing furthermore GFP-Atg8 (representing the PAS) were starved in SD-N for 5 hours and examined under the DeltaVision microscope (equipped with FITC and TRITC filter).

(B) More than 100 cells were monitored for occurrence of the PAS at the nucleolar part of the nucleus. $22.9 \%$ of the PAS dots in close proximity to the nucleolus were observed $(\mathrm{SEM}=7.5 ; \mathrm{n}=2)$. 


\section{Discussion}

The yeast, Saccharomyces cerevisiae, provides many advantages as a model organism for scientific research, such include the fast growth combined with the easy cultivation and nonpathogenicity. Furthermore, yeast cells are easy to handle for transformation (see 3.3.2/3.3.3). A wide range of yeast vectors is commercially available (e.g. $2 \mu$ plasmids), which allow the expression of yeast and also foreign genes. S. cerevisiae provides the advantage of using homologues recombination for the precise deletion or modification of single genes (see 3.3.13/3.3.14). Standard methods for measuring gene expression, the identification of proteinprotein interaction, as well as the analysis for functional relationships between different genes and/or their products (e.g. SGA methodology; see 3.2.11) allow the identification of cellular functions of single genes/proteins or their complexes. The resulting information can in most cases be transferred to higher eukaryotic cells, making $S$. cerevisiae a very useful model organism.

\subsection{Synthetic Genetic Array (SGA)}

The SGA methodology is a powerful high-throughput technique to explore synthetic lethality allowing the systematic production of double deletions by crossing the entire genome deletion library available for $S$. cerevisiae (DMA) with a deletion mutant of interest (query strain). When two genes show a synthetic lethal interaction, it often reflects that the gene products impinge on the same essential function (A. Tong and C. Boone (2007) not published, see: www.utoronto.ca/boonelab/sga_technology).

To benefit from the optimised conditions for the SGA methodology (Tong, 2001; Tong and Boone, 2006), the strain Y8205 (Boone Lab; University of Toronto, Canada; www.utoronto.ca/boonelab) was used for the generation of the query strains in this thesis. Y8205 carries a range of different genetic advantages such as a STE3pr-LEU2 reporter, which is activated only in MAT $\alpha$ cells. After mating of the query strain with Y8205, the kanamycin resistance cassette should be switched into a nourseothricin resistance to enable the use of the deletion library (KanMX6). As the method described in literature (Tong and Boone, 2006) resulted not in a successful switch, the NAT resistance cassette was amplified by PCR (using the plasmid pAG25 as template and corresponding primers) and inserted by homologues recombination. The comprised STE3pr-LEU2 reporter enabled the further selection for only haploid MAT $\alpha$ meiotic progeny using medium lacking leucine. 
This modified query deletion mutant was used for the presented screen with the entire deletion mutant library available for $S$. cerevisiae (Euroscarf). The different selection steps after mating and sporulation of each different deletion combination was simplified by further genetic attributes, resulting out of crossing with the Y8205 strain. The STE2pr-HIS3 reporter, mainly induced in Mat a cells (A. Tong and C. Boone (2007) not published, see: www.utoronto.ca/ boonelab/sga_technology), allowed the selection of haploid progeny of mating type a (Mat a) using medium lacking histidine. Furthermore, the deletion of CAN1 and LYP1 also ensured the selection for haploid cells of mating type a by the elimination of diploids (Mat $\mathrm{a} / \alpha)$ and growth inhibition of false positive Mat a/a diploid mutants. CAN1 encodes an arginine permease that allows canavanine, a toxic analogue for arginine, to enter and kill cells (Hoffmann, 1985). LYP1 encodes a lysine permease that allows, similar to canavanine, thialysine (a toxic analogue for lysine) to enter the cells resulting in death of the cells (Sychrova and Chevallier, 1993). Diploid cells (mat a/ $\alpha$ ) still carry a wild-type copy of the CAN1 and LYP1 genes and are killed by the treatment with $100 \mu \mathrm{g} / \mathrm{l}$ canavanine and 100 $\mu \mathrm{g} / 1$ thialysine. A possible mitotic recombination, resulting in a crossover event of the Mat locus and therefore in Mat a/a diploids, is also repressed under canavanine and thialysine treatment (Tong and Boone, 2006). The protocol recommended by Tong and Boone (2006) was furthermore modified in the step of sporulation, by omitting the proposed $200 \mu \mathrm{g} / \mathrm{l} \mathrm{G} 418$, to reduce false positive kanamycin resistance mutants. Using these optimisation strategies, the potential of false negative SGA hits was drastically reduced. Nevertheless, the screen procedure containing at least 5 pinning steps, led to false positive synthetic lethal double deletions, mainly caused by problems with pinning of the spores on selection medium and sporulation defects in some deletion strains. 53 plates containing 384 colonies $(2 \mathrm{x} 192$ different combinations) had to be analysed for positive double deletions, represented by synthetic lethality (Figure 15, Table 18, chapter 4.1). Double deletion strains of interest were furthermore examined by separate re-mating of the single deletion strains, followed by sporulation, separation of the tetrads and analysis of the growth phenotype.

In this study it was screened for synthetic lethality of a double deletion of $\operatorname{atg} 21 \Delta$ $x_{(D M A)} \Delta$. Atg21 and its homologues Atg18 and Ygr223c are phosphoinositide binding proteins that contain seven WD-40 repeats and are predicted to fold as seven bladed B-propellers. WD40 repeat proteins are thought to serve as stable platforms for protein-protein interaction, thereby mediating the assembly of multiprotein complexes. Atg18, Atg21 and Ygr223c are required for different subsets of autophagic processes. Atg21 is strictly required for the Cvtpathway, efficient unselective autophagy and PMN (Barth, 2001; 2002; Krick, 2006; Krick, 
2008a). Atg21 is further involved in lipidation and effective recruitment of Atg8 to the PAS during the Cvt pathway. Atg18 localises to the PAS and the vacuolar membrane. Its localisation requires Atg2 and PtdIns3P, synthesised by the Vps34 complex I, containing Atg14. A PAS localisation of Atg21 and Ygr223c is still under investigation. All three homologues are recruited to the endosomal compartment via PtdIns3P, generated by the Vps34 complex II, containing Vps38. Therefore an endosomal function of all three homologues distinct from autophagy has been suggested (Dove, 2004; Meiling-Wesse, 2004; Strømhaug, 2004; Krick, 2008a).

To get more hints for a potential role of Atg21 inn autophagy and its putative role in endocytosis, which might be shared with Atg18 and Ygr223c, the BY4741 atg21 $\Delta$ strain was chosen as query strain in the SGA screen. The double deletions strains $\operatorname{atg} 21 \Delta \mathrm{vac} 1 \Delta$ and $\operatorname{atg} 21 \Delta \operatorname{atg} 27 \Delta$ resulted in a synthetic lethal phenotype. These double deletion strains of interest were further examined by separate remating of the single deletion strains, followed by sporulation, separation of the tetrads and evaluation of the growth phenotype. The generated

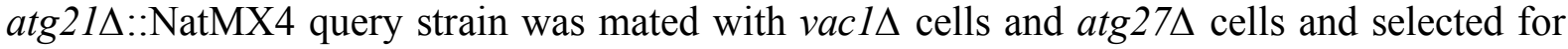
the haploid double deletion progeny by using the conditions of the original screen. To exclude the modifications needed for the SGA methodology and provide a further control, $\operatorname{atg} 21 \Delta::$ KanMX6 cells and atg27D::HIS3 cells of the WCG background were used in addition to the BY4741 background used in the screen.

Vac1 is an effector of the Rab GTPase Vps21 at the endosome. It interacts with the GTPbound Vps21 that functions in Golgi-to-endosome transport and with Vps45, bound on its tSNARE Pep12, at the endosomal membrane. Therefore Vac1 might functionally couple

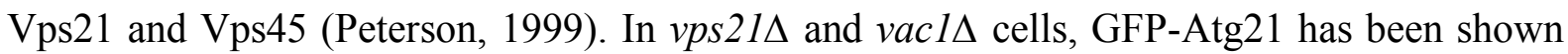
to be released from the endosomal compartment into the cytosol. The localisation of GFPAtg18, GFP-Atg21 and GFP-Ygr223c at the endosome has been shown to require the Vps

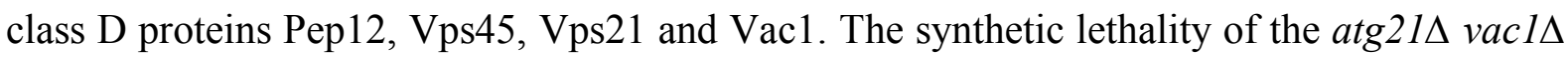
double deletion in the screen, in combination with the involvement of these endosomal components, especially $\mathrm{Vac} 1$, in the localisation of Atg21 and its homologues, point to a function of Atg21 in a protein trafficking pathway at the endosome (Krick, 2008a).

Atg27 has been described as PtdIns (3) phosphate-binding protein and a downstream effector of Vps34 (Wurmser and Emr, 2002). Vps34 is a main component of the two phosphatidylinositol 3-kinase complexes in S. cerevisiae, needed for autophagy (complex I) and the Vps pathway (complex II) (Figure 4, chapter 2.2.1.3). More recently, a sequencing error leading to a longer Atg27 protein sequence has been published. This Atg27 version does 
not require the function of Vps34 or the binding to PdtIns3P for localisation at the PAS (Yen, 2007). Atg27, besides Atg23, is required for the cycling of Atg9 between the preautophagosomal structure, mitochondria, and the Golgi complex by interaction with Atg9 (Tucker, 2003; He, 2006; Yen, 2007). Furthermore, Atg27 is required for the Cvt pathway and efficient unselective autophagy, as described for Atg21 (Wurmser and Emr, 2002; Yen, 2007). These functional similarities in phenotype and the potential involvement of both proteins in protein trafficking pathways, makes a synthetic lethality highly reasonable.

Both double deletions, atg2 $1 \Delta \operatorname{vac} 1 \Delta$ and $\operatorname{atg} 21 \Delta \operatorname{atg} 27 \Delta$, were of high interest for further investigations. Unfortunately, these double deletions did not show a synthetic lethal phenotype in the experiments of separate remaiting, done for confirmation of the screen results. Hence, both double deletions had to be valuated as false positive matches.

Even though this SGA method is simplified by all the genetic attributes and the work with the screen robot was very diversified, the procedure was not such effective as expected. The use of the robot should have facilitated the experiments by reducing the time of the screen. Unfortunately the robot needed to be monitored during the whole procedure. Single pins tended to fix in wrong positions, leading to the loss of colonies. Furthermore, irregularities in thickness or even slight disarrangement of the OmniTray plates in the gadget led to the loss of cells. Therefore the work with the robot was very time consuming. As an alternative to the robot, manual pin tools were used. Even though using these pin tools did not significantly reduce or extend the duration of the screen, they allowed better time management for other experiments. 


\subsection{Establishment of a novel assay to easily measure unselective autophagy} using Pgk1-GFP

For the detection of unselective (macro-) autophagy by western blot, Atg8 and its mammalian homologue LC3 are standard marker proteins (Klionsky, 2007). In this study the cytosolic 3phosphoglycerate kinase Pgk1 was introduced as a novel and convincing marker protein in $S$. cerevisae (Welter, 2010).

Unselective autophagy is a ubiquitous process among eukaryotes and is one of the major degradative pathways (Reggiori and Klionsky, 2002). It has been shown to be involved in several diseases like Alzheimer (Nixon, 2007; Jaeger and Wyss-Coray, 2010), Parkinson's (Xilouri, 2008) or Huntington's disease (Shibata, 2006) and is therefore of high interest in the scientific community. Methods for monitoring the autophagic rate were established in $S$. cerevisiae and also mammalian cells. The common method, also used in this study (see 3.4.5.1), is to monitor the autophagic degradation of GFP-Atg8 by western blot (MeilingWesse, 2002) similar to the standard method for monitoring mitophagy by detecting the degradation of Om45-GFP (Kanki, 2009a).

The autophagic degradation of GFP-Atg8 includes both, unselective and selective autophagic variants. Atg8 is known to be needed for autophagosome formation and is selectively recruited during the assembly of the autophagic core machinery. Under nutrient rich conditions, Atg8 is found intracellularly in considerable amounts, but its expression increases drastically under nutrient starvation conditions. It is conjugated to $\mathrm{PE}$ and thus coupled to the inner and outer membrane of the forming autophagosome. The amount of Atg8 localised to the outer membrane of the autophagosome is cleaved off by the specific protease Atg4, the part of Atg8 localised at the inner autophagosomal membrane is transported to the vacuole where it is degraded (Kirisako, 1999; Ichimura, 2000; Kirisako, 2000). Atg8 is therefore selectively recruited to the PAS during autophagosome biogenesis, thus the autophagic rate, measured via degradation of GFP-Atg8 might be overestimated. Furthermore GFP-Atg8 is degraded via both, unselective and selective autophagy. Hence, GFP-Atg8 is probably a biased marker for the quantification of the unselective macroautophagic rate.

In contrast, Pgk1 (3-phosphoglycerate kinase), the key enzyme in glycolysis and glyconeogenesis, represents a more unselective cytosolic cargo of autophagy and was therefore introduced as a novel marker protein for the detection of unselective bulk autophagy in this thesis (Welter, 2010). 
Figure 17 (A) (chapter 4.2) presents western blot analysis using the marker protein Pgk1GFP. After nutrient starvation and thereby induction of autophagy, wild type cells showed a clear autophagic degradation of the protein detected by accumulation of free GFP. In contrast the two negative controls atg $1 \Delta$ cells, deficient in autophagy, and pep $4 \Delta$ cells, disabled in intravacuolar proteolysis, showed no free GFP. Atg21 is essential for the maturation of proaminopeptidase I under non-starvation conditions (Cvt pathway), while unselective autophagy is only reduced in atg21 $1 \Delta$ cells (Barth, 2002). As expected, a clear reduction in signal intensity for free GFP in $A T G 21$ deleted cells, compared to wild type cells was detected. To quantifiy western blot signals, the ratio of free GFP to Pgk1-GFP in wild type cells after 6 hours of nitrogen starvation was set to $100 \%$. The clear reduction in bulk autophagy in atg2 $1 \Delta$ cells was calculated to less than $20 \%$ (Figure 17 (A) and (B), chapter 4.2).

The autophagic rate of $A T G 21$ deleted cells was originally published to be reduced to $35 \%$ (Meiling-Wesse, 2004). This slight discrepancy is most likely the result of using Pgk1-GFP as selective bulk autophagy substrate instead of the autophagy substrate GFP-Atg8. Although the requirement of Atg21 for mitophagy, the selective degradation of mitochondria, is still under discussion (Kanki, 2009b; Okamoto, 2009), Atg21 is known to be essential for the selective Cvt pathway and PMN (Barth, 2002; Krick, 2008b). The use of Pgk1-GFP as marker protein in degradation assays therefore reflects the rate of unselective bulk autophagy in a more acurate manner than the use of GFP-Atg8.

Additional microscopic analysis using cells expressing Pgk1-GFP were performed (Figure 18, chapter 4.2) and support the data of the western blot experiments using the same strains. To avoid the degradation of autophagic bodies by proteinase B, $1 \mathrm{mM}$ PMSF was added to all strains. Wild type cells showed, besides the green fluorescent cytosol, clear green punctate structures, representing the autophagic bodies, moving around inside the vacuole. In contrast, ATG1 deleted cells did not contain these intravacuolar vesicles. In pep $4 \Delta$ cells an accumulation of autophagic bodies inside the vacuole was clearly visible. As already indicated by western blot quantification, in $\operatorname{atg} 21 \Delta$ cells the amount of green fluorescent autophagic bodies was significantly reduced.

The utility of the Pgk1-GFP degradation assay can be furthermore accentuated by comparison with other common methods, such as the monitoring of autophagy by a radioactive assay, which requires the radioactive labelling of amino acids in cellular proteins (Prick and Thumm, 2008). Samples are taken at different time points of starvation and are precipitated with TCA. The TCA-soluble radioactivity, which corresponds to the degradated 
proteins, is determined using a scintillation counter. Although this method provides reliable results, it is time consuming, expensive and demands for experience in handling. A further method is the use of an enzymatically inactive cytosolic variant of vacuolar alkaline phosphatase (Pho8 $\Delta 60)$, which only becomes active after its transport to the vacuole by unselective autophagy followed by maturation inside the vacuole (Noda, 1995). The autophagic rate can be determined by the measurement of enzyme activity. This assay requires the chromosomal deletion of $\mathrm{PHOl3}$ for reduction of background activity and

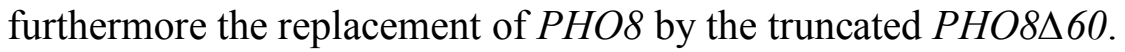

In summary, in comparison to other methods, the introduced Pgk1-GFP degradation assay exhibits an appropriate method concerning the costs, the required practise in handling and the required of time. 


\subsection{A complex of Ccd48, Shp1 and Atg8 is needed for autophagosome} biogenesis in yeast

A recent publication, also presenting results of this study, introduces a new role for Cdc48 and its cofactor Shp1 in autophagosome biogenesis (Krick, 2010).

Until now, the mechanism of autophagosome biogenesis is poorly understood. Two of the proposed models for elongation of the phagophore by homotypic or heterotypic fusion events (Longatti and Tooze, 2009) morphologically remind on nuclear envelope expansion and postmitotic Golgi reassembly (Meyer, 2005). One model proposes that the required membrane for the growing phagophore is delivered through vesicular transport from a membrane source to the PAS. Therefore a part of the fusion machinery, also required for other vesicular fusion events would be needed, including motor proteins, Rab GTPases, their effectors and SNARE proteins. Up no now, no SNAREs or autophagy-specific Rab effectors have been identified (Ishihara, 2001; Longatti and Tooze, 2009). The other model proposes that the autophagosome is assembled by homotypic fusion of special autophagy vesicles. These vesicles are either generated in response to autophagy induction or are constantly present in the cytoplasm (Reggiori, 2004b; Longatti and Tooze, 2009; Mari, 2010). Therefore a special autophagy fusion machinery, or also a common homotypic fusion machinery instead of a heterotypic one, might be required. Such a fusion event is considered to be mediated by Cdc48 and its cofactor Shp1.

\subsubsection{Shp1 is the exclusive cofactor of Cdc48 needed for autophagy}

In contrast to another publication (Ishihara, 2001), Cdc48 was shown to be required for autophagy in previous experiments in the Thumm laboratory by testing the temperature sensitive mutant Cdc48-3 (Bremer, 2009). At the permissive temperature, starved cells showed normal unselective autophagy, but after the shift to the nonpermissive temperature of $38{ }^{\circ} \mathrm{C}$, the GFP-Atg8 degradation assay has shown a strict block in autophagy, while the cellular survival has not been affected at $38^{\circ} \mathrm{C}$ (Bremer, 2009; Krick, 2010).

These results made it necessary to test known cofactors of Cdc48 in the GFP-Atg8 degradation assays. Preliminary data already pointed to Ubx1/Shp1 as the only cofactor of Cdc48 needed for autophagy (Bremer, 2009). Ubx1/Shp1 is a member of the UBX protein family, consisting of 7 UBX proteins, described as general Cdc48 interacting proteins. They interact with Cdc48 via their UBX domain (Hartmann-Petersen, 2004; Schuberth and 
Buchberger, 2008) and three of the proteins further possess an amino-terminal UBA domain, which has been shown to bind ubiquitinated proteins in vivo (Schuberth, 2004).

Indeed, the analysis of these cofactors using the GFP-Atg8 degradation assay in this thesis, confirmed Ubx1/Shp1 as the exclusive cofactor of Cdc48 needed for autophagy (Figure 19, chapter 4.3.1). SHP1 deleted cells were additionally tested by using the established Pgk1-GFP degradation assay (Welter, 2010), also leading to the result that Shp1 is essential for autophagy (Figure 23, chapter 4.3.2).

Slide effects on proteasomal degradation of ubiquitinated proteins were reported for Shp1, therefore an involvement of the ubiquitin proteasome system had to be tested. Additional cofactors of Cdc48 were also tested for confirmation of previous experiments (Bremer, 2009). No autophagic defect was detected in cells lacking the substrate recruiting factor Ufd1, the substrate processing cofactors Ufd2 and Ufd3, the ubiquitin ligase Ufd4 or the proteasome regulator Ufd5 (Figure 20, chapter 4.3.1). Furthermore, cells lacking the ERAD component Der1 and the substrate processing Cdc48 cofactor Otu1 were tested. Otu1 is distantly related to mammalian VCIP135, which is the necessary cysteine protease for triggering membrane fusion in mitotic Golgi reassembly in mammals by deubiquitinating an unknown fusion

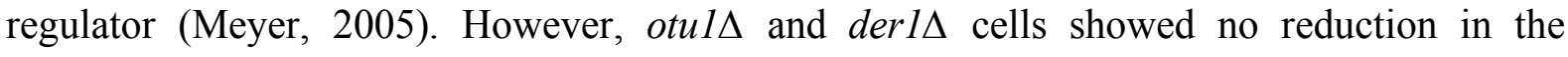
autophagic rate (Figure 21, chapter 4.3.1). The proteasome-deficient mutant, pre1-1 pre2-2 has been tested in previous experiments in the Thumm laboratory, without showing any effect on autophagy (Krick, 2010). All these data argue against a need for the ubiquitin proteasome system for the autophagic function of Cdc48 and its cofactor Shp1.

In this study, these findings were furthermore confirmed in a GFP-Atg8 degradation assay of doa4s cells. The deletion of Doa4 depletes the cellular ubiquitin levels, and causes many cellular defects, including the loss of efficient MVB (multi vesicular body) sorting of some integral membrane proteins (Ren, 2008). The autophagic degradation of GFP-Atg8 was not drastically decreased in doa4t cells (Figure 22, chapter 4.3.1).

Together, the previous results and the results in this thesis showed that Cdc48 and Shp1 are required for unselective autophagy independently of ubiquitination and proteasomal degradation.

Furthermore, microscopic analysis showed that in contrast to pep $4 \Delta$ cells, shp $1 \Delta$ cells failed to accumulate autophagic bodies in the vacuole (Figure 25, chapter 4.3.3). The treatment of the cells with $1 \mathrm{mM}$ PMSF, which inhibits the vacuolar proteinase B leading to an accumulation of autophagic bodies in wild type cells, showed the same result. These results led to the assumption that no autophagosomes reach the vacuole in $\operatorname{sh} 1 \Delta$ cells. These results 
are consistent with previous fluorescence microscopic analysis, which have demonstrated that the autophagic cargo GFP-Atg8 does not reach the vacuole in shpls cells. Even under PMSF treatment, GFP-Atg8 positive autophagosomes have not accumulated in the vacuole (Krick, 2010).

Normal maturation of CPY in shpls cells of different growth phases and under starvation supported to the conclusion that vacuolar proteolysis is not significantly disturbed by the deletion of SHP1 (Figure 24, chapter 4.3.2). These results raised the idea that this deletion causes a defect in autophagosome formation or their vacuolar fusion. A protease protection assay has indicated a defective autophagosome biogenesis or closure (Krick, 2010). Moreover the maturation of Ape1, which was not impaired in shpls cells (Figure 24, chapter 4.3.2), indicated a function of Shp1 in the elongation of the phagophore. The core protein Atg8 is known to be crucial for the control of autophagosomal size (Nakatogawa, 2007; Xie, 2008b). Therefore it was tested if the deletion of SHP1 has an effect on the localisation of Atg8 at the pre-autophagosomal structure or its lipidation, which both are important steps while initiation of the autophagic process. The recruitment to the PAS was analysed by microscopy experiments using the PAS marker protein GFP-Atg8 and, as further control, the PAS marker protein GFP-Atg9 (Reggiori, 2004a; Klionsky, 2007). Cells expressing GFP-Atg8 were starved and PAS structures, represented by clear green punctate structures in close proximity to the vacuole, were counted. PAS dots were observed in $33 \%$ of wild type cells, $86 \%$ of $\operatorname{atg} 1 \Delta$ cells and $42 \%$ of $\operatorname{shp} 1 \Delta$ cells. Additionally, $49 \%$ of shp $1 \Delta$ cells expressing GFP-Atg9

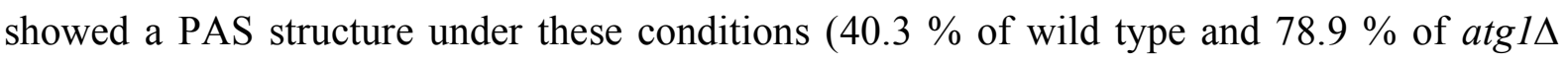
cells). This wild type-like rate of PAS structures indicated, that the formation of the PAS is not influenced by Shp1 (Figure 26 (A) and (B), chapter 4.3.4). Furthermore, the lipidation of Atg8 was not impaired in shp1 $\Delta$ cells (Figure 27, chapter 4.3.5). The increased level of Atg8, observed in shpls cells, might be caused by the autophagic defect and the resulting enrichment of Atg8 in these cells.

\subsubsection{Shp1 interacts with Atg8}

In the model for mitotic Golgi reassemby in mammals, ubiquitin has been shown to be an essential factor (Meyer, 2005). Previous experiments in the Thumm laboratory has shown that ubiquitin is not needed for autophagosome formation (Krick, 2010). Furthermore, autophagosome formation has been shown to require the ubiquitin-like protein Atg8 (Nakatogawa, 2007). Therefore the ubiquitin-like protein Atg8 was thought to replace 
ubiquitin in the fusion complex required for autophagosome formation and therefore to bind to Shp1.

An interaction of Shp1 and Atg8 has already been shown by coimmunoprecipitation and pulldown experiments using recombinant GST-Atg8 (Krick, 2010). In this study, this interaction was proven by pull down experiments using recombinant GST-Shp1 constructs.

Three different constructs were generated: full length GST-Shp1, GST-Shp1 lacking the C-terminal UBX domain and GST-Shp1 lacking the N-terminal UBA domain. The UBX domain is known to be a general Cdc48/p97-binding module (Hartmann-Petersen, 2004) and is described as a distant homologue of ubiquitin itself. Therefore Cdc48/p97 can either bind directly to ubiquitin or the UBX domain of the corresponding protein (Buchberger, 2001). The UBA domain has been shown to bind to ubiquitinated proteins in vivo (Schuberth, 2004). Furthermore, the human homologue of Shp1, p47, requires the binding of ubiquitinated proteins via the UBA domain for efficient p97/p47-mediated Golgi membrane function in vitro (Meyer, 2002). To prove the interaction of Atg8 with Shp1, and its dependence on the UBA or UBX domain, three different constructs (GST-Shp1 full length, GST-Shp1-UBX and GST-Shp1-UBA) were used for pull down experiments (Figure 28 (A) and (B), chapter 4.3.6).

The use of the standard protein purification protocol for the recombinant GST-Shp1 constructs resulted in their degradation. This degradation might be caused by a freezing step in the purification protocol and the followed lysozymal cell disruption using BL21 (DE3) pLys cells. Therefore an alternative purfication was tested. The constructs were transformed in BL21 RIPL cells (Table 8, chapter 3.1.2) and the cell disruption was done by french press (see 3.4.6.4). The cells were treated on ice and freezing was avoided. This optimisation led to a decreased degradation of the recombinant proteins.

The recombinant GST-Shp1 proteins were used for pull down experiments with native cell extract of pep $4 \Delta$ cells expressing GFP-Atg8, to avoid vacuolar proteolysis (Figure 28 (A), chapter 4.3.6). In a second pull down $\operatorname{atg} 4 \Delta$ atg $8 \Delta$ cells expressing GFP-Atg8-FG were used for cell extract preparation (Figure 28 (B)). Atg4 removes the carboxy-terminal arginine of Atg8 to allow coupling to PE. By the use of the Atg8-FG construct, which lacks this arginine, coupling to PE is independent on Atg4. This allows the use of an atg4 $\Delta$ strain, perhaps stabilising the Atg8-PE-Shp1 complex, which would be otherwise decomposed by Atg4 (Kirisako, 2000). Atg8-PE is described to mediate hemifusion during autophagosome formation most likely by mediating oligomerisation (Nakatogawa, 2007). Former experiments led to the suggestion that Shp1 might preferentially interact with Atg8-PE (Krick, 2010). The 
postulated interaction of Atg8 with Shp1 might be enhanced in $\operatorname{atg} 4 \Delta \operatorname{atg} 8 \Delta$ cells expressing GFP-Atg8-FG. All three different GST-Shp1 constructs showed an interaction with Atg8 in the pull down experiments. Interestingly, the recombinant GST-Shp1 lacking the N-terminal UBA domain showed a significant stronger interaction with Atg8 in five independent experiments. This observation might be explained by the inability of the GST-Shp1 construct lacking the UBA domain to bind ubiqutin. Using the full-length GST-Shp1 construct, Atg8 and ubiquitin might compete for binding to Shp1. Hence, Shp1 does not bind Atg8 via the UBA domain. In previous experiments it has been shown that the autophagic activity is not obviously affected by the depletion of the UBA domain (Krick, 2010).

Previous experiments, done in the Thumm laboratory and data of this thesis led to the model that the $\mathrm{AAA}^{+}$ATPase Cdc48 and its cofactor Shp1/Ubx1 are essential factors for autophagosome biogenesis (Figure 7, chapter 2.2.5.1.1) (Krick, 2010). Atg8 may act as a fusion regulator, which must be extracted by the $\mathrm{AAA}^{+}$ATPase Cdc48 and its adapter Shp1 from a complex with a fusion mediator. In this model, Atg8 replaces ubiquitin in a membrane fusion complex during autophagosome formation. The deubiquitination by the cysteine protease VCIP135 in mitotic Golgi reassembly (Meyer, 2005) might be replaced by the use of the cysteine protease Atg4, which delipidates Atg8-PE and leads to a release of the complex for the initiation of the fusion event.

Notably also PMN, the selective degradation of portions of the nucleus by microautophagy has been shown to require Cdc48 and Shp1 (Bremer, 2009). 


\subsection{Insights into mitophagy in S. cerevisiae}

Mitochondria, the site of ATP production in aerobic cells consequently are at the same time the major source of reactive oxygen species (ROS), which damage mitochondria and also other organelles. Dysfunctional mitochondria therefore enhance oxidative stress and may also lead to the scattering of the membrane potential across the inner membrane, resulting in induction of cell death by the release of pro-apoptotic proteins (Kroemer, 2007; Tatsuta and Langer, 2008). Thus, a quality control for mitochondria is essential for cellular survival.

In humans the consequences of mitochondrial dysfunction are linked to ageing and neurodegeneration. Mitochondrial dysfunction has been shown to be involved in Parkinson's disease (PD) several decades ago (Mizuno, 1989), leading to the conclusion that mitochondrial quality control is indispensable for cellular health. The selective degradation of mitochondria by autophagy may represent such a quality management in the cell.

Indeed in the last few years, mitophagy was shown to be one of the major degradative pathways in conjunction with PD. The E3 ubiquitin ligase Parkin, which has no homologue in yeast, triggers the removal of damaged mitochondria. Its dysregulation is therefore linked to PD (Geisler, 2010). During mitophagy, Parkin has been shown to be translocated from the cytosol to uncoupled or depolarised mitochondria (Narendra, 2008). The induction of Parkinmediated mitophagy is dependent on the activity of PINK1, a kinase, which spans the outer mitochondrial membrane (Narendra, 2008; Narendra, 2010a). The interaction of PINK1 with Parkin was found to be significantly enhanced after depolarisation of mitochondria (Geisler, 2010). After the translocation of Parkin to mitochondria, Parkin mediates the linkage of ubiquitin at Lys 27 and Lys 63 to mitochondrial substrates. One protein discussed to represent such a substrate, is the voltage-dependent anion channel 1 (VDAC1) (Geisler, 2010; Narendra, 2010b). Subsequently, ubiquitin receptors have to be recruited to mitochondria. Such a ubiquitin binding adaptor is p62 (Geisler, 2010; Narendra, 2010b), which recruits ubiquitinated cargo into autophagosomes by binding to LC3 (the mammalian homologue of Atg8) (Pankiv, 2007). Furthermore, in mammalian cells, the mitofusins MFN-1 and MFN-2 have been shown to be ubiquitinated in a PINK1- and Parkin-dependent manner, suggesting a proteasomal degradation which furthermore leads to a block in mitochondrial fusion machinery and an autophagy-dependent degradation of mitochondrial fragments (Gegg, 2010). In Drosophila, PINK1 is also required for the translocation of Parkin to dysfunctional mitochondria and the resulting degradation initiated by the ubiquitination of the profusin factor Mfn (Ziviani, 2010). 
Also in $S$. cerevisiae studies in the last few years provided more insight into mitophagy. Two independent genetic screens identified the mitochondrial-anchored transmembrane receptor protein Atg32, which is exclusively required for mitophagy (Kanki, 2009c; Okamoto, 2009). Typical for an autophagic cargo receptor, Atg32 interacts with Atg8 via a highly conserved WXXI motif in its cytosolic (N-terminal) region (Kanki, 2009c; Okamoto, 2009). Atg19, which selectively binds to cargo proteins in the Cvt pathway, interacts via a WXXL motif with Atg8 (Shintani, 2002). The mammalian p62 binds ubiquitinated cargo and interacts with LC3, the mammalian homologue of Atg8, via a WXXL motif (Pankiv, 2007). Therefore, a similar function in cargo recognition and Atg8 interaction is plausible for Atg32 in mitophagy, especially because of a nitrogen starvation induced interaction with Atg11, which is necessary for selective cargo reporter recognition in the Cvt pathway and pexophagy (Kim, 2001).

In S. cerevisiae, the established Om45-GFP degradation assay presents an assured method for monitoring mitophagy, but requires the chromosomal GFP-tagging of Om45 (Kanki, 2009a). To enable the detection of mitophagy using a plasmid-encoded marker protein, similar to the marker proteins GFP-Atg8 or Pgk1-GFP for the detection of unselective autophagy, plasmids encoding the mitochondrial proteins Om45-GFP and Idh1-GFP were constructed in this study. The use of these plasmids led degradation of the marker proteins independent from Atg1 or Pep4 (data not shown). Therefore mito-GFP was constructed by the insertion of the mitochondrial targeting sequence Su9-mt-DHFR into the vector pUG35. Similar to plasmids used in other studies (Tal, 2007; Okamoto, 2009), the mito-GFP of this study showed a localisation at mitochondria (Figure 31 (A), chapter 4.4.3) and was degraded in an autophagydependent manner after 48 hours growth in medium containing lactate as sole carbon source (Figure 31 (B)), indicating the induction of mitophagy after exhaustion of the carbon source. Due to the use of the pUG35 vector, the expression of mito-GFP is under control of a MET25 promoter, thus all experiments were done in S. cerevisiae of WCG or BY4742 background, which allows growth in medium lacking methionine. 


\subsubsection{Differentiation between mitophagy of superfluous or damaged mitochondria}

To monitor mitophagy in S. cerevisiae, Kanki et al. (2009a) described two different conditions to induce mitophagy. On one hand, the induction by amino acid starvation, which means the shift of the cells from a nonfermentable carbon source (lactate medium), leading to the proliferation of mitochondria, to nitrogen starvation medium containing glucose. The other method involves the induction at post-log phase meaning a prolonged incubation for up to three days in medium containing $2 \%$ lactate as sole carbon source. Recent publications, using both conditions, have shown that Uth1, a protein primarily postulated as essential for mitophagy (Kissová, 2004), is not needed for the selective degradation of mitochondria (Kanki, 2009b; Okamoto, 2009). This fact was also confirmed in this study (data not shown). Nevertheless, the comparison of both studies lead to some discrepancies concerning the need of Atg13 or Atg21 for mitophagy (Kanki, 2009b; Okamoto, 2009).

In regard to the discrepancies, the initiation of different mitophagy processes can not be excluded, meaning recognition and determination of superfluous, aged or highly damaged mitochondria (by external or internal stress factors, e.g. reactive oxygen species). Therefore, different conditions to induce mitophagy were tested and established in this study. After growth for up to 72 hours in medium containing $2 \%$ lactate, mitochondria are aged and might be severely damaged. The mitophagic degradation was monitored by western blot analysis and a time-dependent increase of free GFP in wild type cells was shown. In the atg184 control cells, deficient for autophagy (Barth, 2001), and atg32s, deficient for mitophagy (Kanki, 2009b; Okamoto, 2009), no signal for free GFP was detected (Figure 32, chapter 4.4.4). These observations were consistent with published results (Okamoto, 2009).

Superfluous mitochondria, which generate reactive oxygen species (ROS) as a consequence of metabolic processes, might present a specific cargo for autophagy, to ensure cellular survival. In the literature, degradation of mitochondria was further initiated by the shift to SD-N medium containing glucose (Kanki and Klionsky, 2008), the common method to induce autophagy (Takeshige, 1992). As the switch from medium containing $2 \%$ lactate to SD-N medium did not result in measurable mitophagic degradation in this study (data not shown), $0.2 \mu \mathrm{g} / \mathrm{ml}$ rapamycin was added to the cells of interest. The addition of rapamycin leads to the initiation of autophagy, by inhibition of the TOR kinase (Noda and Ohsumi, 1998). Indeed, in contrast to $\operatorname{atg} 18 \Delta$ and $\operatorname{atg} 32 \Delta$ control cells, wild type cells showed an increased level of mitophagy after treatment with rapamycin (Figure 33, chapter 4.4.4). 
A slight rate of unselective autophagy was already detectable in wild type and $\operatorname{atg} 32 \Delta$ cells before treatment with rapamycin. This observation led to the conclusion that unselective autophagy is already induced after $14-16$ hours of growth in preculture medium containing 2 $\%$ lactate.

As already mentioned, mitochondria are a major source of reactive oxygen species (ROS), therefore a qualitiy control of mitochondria is indispensable. Recent publications postulated an induction of autophagy by $\mathrm{ROS}$ (e.g. $\mathrm{O}_{2}{ }^{-}$or $\mathrm{H}_{2} \mathrm{O}_{2}$ ) (Kiffin, 2006; Chen, 2009), suggesting a function of mitochondrially generated $\mathrm{H}_{2} \mathrm{O}_{2}$ in regulating the lipidation of Atg8 via the Atg4-activity (Scherz-Shouval and Elazar, 2007). ROS are generated endogenously in cells by reduction of oxygen and can lead to the production of more ROS. They are highly damaging towards cellular constituents, e.g. DNA, proteins and lipids after an increase of concentration beyond the antioxidant buffering capacity. To protect the cellular constituents, yeast (and other organisms) has developed a ubiquitous enzymatic and non-enzymatic oxidant defence system. For example $\mathrm{O}_{2}^{-}$, a product of incomplete one-electron reduction of oxygen which can be converted to $\mathrm{H}_{2} \mathrm{O}_{2}$ by two different superoxid dismutases (SOD). One is the cytoplasmical $\mathrm{Cu} / \mathrm{ZnSod} / \mathrm{Sod} 1$, encoded by $S O D 1$, and the other is the mitochondrial MnSod/Sod2, encoded by SOD2. $\mathrm{H}_{2} \mathrm{O}_{2}$ is furthermore converted to $\mathrm{O}_{2}$ and $\mathrm{H}_{2} \mathrm{O}$ by the gluthathione peroxidase, which uses gluthathione as reductant, or by different catalases. E.g. $\mathrm{H}_{2} \mathrm{O}_{2}$, which is produced by fatty acid $\beta$-oxidation, is converted by the peroxisomal catalase A (encoded by CTA1) (Jamieson, 1998; Chen, 2009).

In this thesis, $\mathrm{H}_{2} \mathrm{O}_{2}$ was tested as external stress factor to induce the selective degradation of dysfunctional mitochondria. Different concentrations of $\mathrm{H}_{2} \mathrm{O}_{2}$ were tested in wild type and $\operatorname{atg} 18 \Delta$ cells expressing mito-GFP. In parallel the survivial rate was analysed, to exclude affects of dead cells. The treatment with $0.05 \mathrm{mM} \mathrm{H}_{2} \mathrm{O}_{2}$ for three hours led to a cell survival rate of $89 \%$ and measurable signal for free GFP in wild type cells but not in atg18 cells, used as negative control (Figure 34 (A) and (B), chapter 4.4.4). An increase of the concentration of $\mathrm{H}_{2} \mathrm{O}_{2}$ from 0.1 to $1 \mathrm{mM}$ led to a decrease in cell survival to $83 \%$ to 63 $\%$. As in former litertature this decrease in viability of wild type cells after exposure to 1.2 $\mathrm{mM} \mathrm{H}_{2} \mathrm{O}_{2}$ appeared more drastically (about $30 \%$ ) (Collinson and Dawes, 1992; Palermo, 2007), $0.05 \mathrm{mM} \mathrm{H}_{2} \mathrm{O}_{2}$ was used in further experiments in this study (Figure 34 (C) and (D), chapter 4.4.4). In contrast to the control cells atg $18 \Delta$ and $\operatorname{atg} 32 \Delta$, the treatment with $\mathrm{H}_{2} \mathrm{O}_{2}$ induced mitophagy in wild type cells. Initiated by growth in lactate medium during preculture, also in this experiment a slight rate of unselective autophagy was detected in wild type and $\operatorname{atg} 32 \Delta$ cells before treatment with $\mathrm{H}_{2} \mathrm{O}_{2}$. 
In further experiments, the ability of paraquat (N, N'-dimethyl-4,4'-bipyridinium dichloride) to induce mitophagy in yeast was tested. This pesticide has already been linked to Parkinson's disease by the damage of mitochondria (Cocheme and Murphy, 2008; Terzioglu and Galter, 2008; Narendra, 2010a). It impairs the mitochondrial function by the acception of electrons from one of the electron carriers, yielding to the production of $\mathrm{O}_{2}{ }^{-}$and $\mathrm{H}_{2} \mathrm{O}_{2}$.

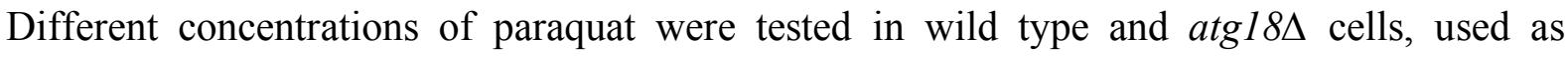
negative control, expressing mito-GFP. Again, in parallel the survival rate was analysed. The addition of 5 to $15 \mathrm{mM}$ paraquat to wild type cells led to a decrase in cell survivial to 80 to 75 $\%$. The treatment with $2 \mathrm{mM}$ paraquat for three hours led to a cell survival rate of $84 \%$ and measurable mitophagy induction in wild type cells, but not in atg18 cells (Figure 35 (A) and (B), chapter 4.4.4). This observation was similar to results in literature, where the treatment of yeast cells with $2.5 \mathrm{mM}$ paraquat has led to a decrease in cell viability to $88 \%$ (Lee and Hassan, 1985).

Hence, $2 \mathrm{mM}$ paraquat was used for further experiments (Figure 35 (C) and (D), chapter 4.4.4). In contrast to the $\operatorname{atg} 18 \Delta$ and $\operatorname{atg} 32 \Delta$ control cells, mitophagy was induced in wild type cells by the treatment with $2 \mathrm{mM}$ paraquat. Initiated by the preculture conditions, also in this experiment a slight rate of unselective autophagy was detected in wild type and atg32 $\Delta$ cells before treatment with paraquat.

The results indicated that mitochondria are severely damaged by the treatment with $\mathrm{H}_{2} \mathrm{O}_{2}$ and paraquat, which further leads to the production of more ROS far beyond the antioxidant buffering capacity of the cell. Damaged mitochondria, which are not immediately degraded, are a risk for the cell and cellular survival. At this point mitophagy supports the oxidant defence system (e.g. SOD and catalases). Recent publications have shown that the addition of paraquat, yielding to the production of $\mathrm{O}_{2}{ }^{-}$and $\mathrm{H}_{2} \mathrm{O}_{2}$, further induces the biosynthesis of the mitochondrial MnSOD/Sod2 (Hassan and Fridovich, 1979), indicating a role in protection of mitochondria from respiration-generated ROS.

To determine if these stressors selectively induce mitophagy, unselective bulk autophagy was monitored in parallel using Pgk1-GFP in all strains tested under the same conditions. A slight rate of unselective bulk autophagy was already induced after 14 - 16 hours of growth in preculture medium containing $2 \%$ lactate. Initial observations indicated a difference between the amounts of free GFP produced by autophagy or mitophagy. But as the use of preculture medium containing $2 \%$ lactate already induced unselective autophagy, it can only be speculated about an exclusive role of paraquat or $\mathrm{H}_{2} \mathrm{O}_{2}$ in the induction of mitophagy. Further investigations, using optimised growth conditions might avoid the induction of unselective 
autophagy and probably prove that the treatment with paraquat specifically induces only mitophagy, but not unselective autophagy.

Studies in $H$. polymorpha have shown, that the shift of methanol-grown cells to glucose medium initiates macropexophagy, while in P. pastoris the same conditions initiate micropexophagy. Ethanol, however, induces macropexophagy in both yeasts (Veenhuis, 1983; Tuttle and Dunn, 1995; Sakai, 2006). Furthermore, in S. cerevisiae, after a shift from medium containing lactate to SD-N medium, a micromitophagic uptake of mitochondria by the vacuolar membrane has been observed by electron microscopy (Kissová, 2007). Together, these results lead to the assumption, that different conditions to induce mitophagy might alternatively cause micro- or macromitophagy.

The different methods to induce mitophagy used in this study, did not result in obvious differences between the tested deletion strains. To elucidate the mechanism of mitophagy in $S$. cerevisiae, further work especially electron microscopy will be needed.

In a screen for yeast deletion mutants defective in mitophagy, done with cells of post log phase in medium containing glycerol, Ccz1 has been identified (Okamoto, 2009). As Ccz1 is further known to be required for the fusion of autophagosomes with the vacuole (MeilingWesse, 2002), these findings might indicate that most of the mitochondria are degraded via a macroautophagic process. Notably, this speculation about a macroautophagic process in glycerol medium, is not contrary to the suggestion that microautophagic degradation of mitochondria is favored by lactate-grown cells (Kissová, 2007).

ATG13 deleted cells were also analysed in this study. Atg13 activates Atg1 in a TORdependent manner and is therefore an essential protein for autophagy, including PMN and the

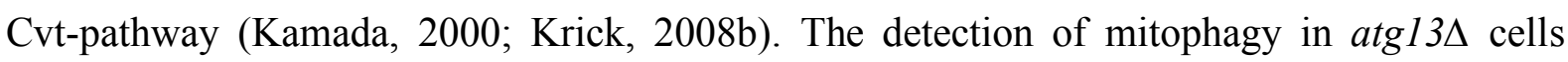
expressing mito-GFP in this study (Figure 32 - 35, chapter 4.4.4), led to the idea that Atg1 can be activated in an Atg13-independent manner under respiratory growth conditions, what has already been observed and discussed in former literature (Okamoto, 2009). Remarkably, another group published an Atg13-dependent initiation of mitophagy (Kanki, 2009b). These discrepancies may be caused by the use of different marker proteins for the degradation assays. On the one hand, using a plasmid similar to the mito-GFP plasmid of this study, has

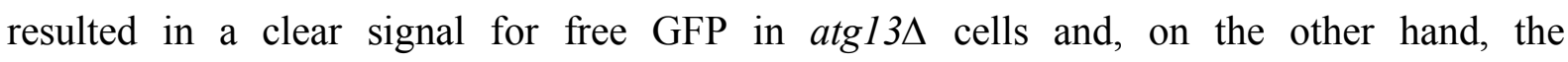
chromosomal GFP-fusion of Om45 in atg13د cells showed no signal for free GFP under starvation. A similar observation was made for atg21 $1 \Delta$ cells in these publications (Kanki, 2009b; Okamoto, 2009). In this study, atg21د cells grown for 72 hours in medium containing 
lactate showed a normal rate of mitophagy (data not shown) confirming the results of Okamoto et al. (2009).

Perhaps, using the plasmid expressed mito-GFP as marker protein is the more sensitive method to measure mitophagy than using chromosomally expressed Om45-GFP, hence a direct comparison of these two marker proteins, using both conditions for the induction of mitophagy within one experiment should shed light on this question.

\subsubsection{Mitophagy is increased in yme1s cells}

Ymel is a subunit of the mitochondrial inner membrane i-AAA protease complex and is responsible for the degradation of unfolded or misfolded mitochondrial proteins (Leonhard, 1996). A loss of the Ymel function leads to more dysfunctional mitochondria, probably inducing an increased rate of mitophagy (Campbell and Thorsness, 1998).

YME1 deleted cells were tested in the mito-GFP degradation assay and showed a drastically increased amount of free GFP after growth for 72 hours in lactate medium resulting in a mitophagic rate of $122 \%$ compared to wild type cells. Already after growth for 24 hours, yme1s cells showed a signal intensity for free GFP of $57 \%$ compared to $13 \%$ in wild type cells (Figure 36, chapter 4.4.5).

This significant 3.5 fold increase in mitophagy after 24 hours represents the selective degradation of damaged mitochondria, caused by unfolded or misfolded mitochondrial proteins in the absence of Yme1. Speculatively, these proteins could be specifically labelled to destignate the mitochondria for degradation.

Nevertheless, these results confirm the observations in former literature, where the, by approximately 3.5 fold, increased ALP acitivity of yme1s cells compared to wild type cells in medium containg glycerol and ethanol has been reflective of selective mitochondrial degradation and not of a general increase in unselective autophagy (Campbell and Thorsness, 1998). 


\subsubsection{Part of the ESCRT machinery is required for mitophagy}

The Vps class E proteins Vps27 and Vps28 have been shown to be needed for the selective piecemeal microautophagy of the nucleus (PMN) (Bremer, 2009). But deletion of VPS27 or VPS28 does not effect the maturation of prApe1 under nutrient rich or starvation conditions (Reggiori, 2004b) or the autophagic degradation of GFP-Atg8 under starvation conditions (Bremer, 2009). This is in contrast to the mammalian system, where autophagic degradation is inhibited in cells depleted of several ESCRT proteins such as the homologue of Vps24 (Filimonenko, 2007).

To test the need of class E proteins for mitophagy, different deletion strains were monitored (Table 19). Deletions of VPS27 (ESCRT-O) and VPS23 (ESCRT-I) were used for mito-GFP and Pgk1-GFP degradation assays. The cells were grown for up to 72 hours in medium containing $2 \%$ lactate as sole carbon source. Both pathways show a strong signal for free GFP in $v p s 27 \Delta$ and also vps $23 \Delta$ cells (Figure 37 (A), chapter 4.4.6). The quantification of signal intensity for GFP of both strains (Figure 37 (D)) led to the conclusion that neither Vps27 nor Vps23 influence the degradation of mitochondria or the unselective bulk autophagy of cytosolic material. In contrast, vps28 cells (ESCRT-I) showed a drastical decrease in mitophagy (38\%), but not in unselective autophagy (77\%) (Figure 37 (B) and

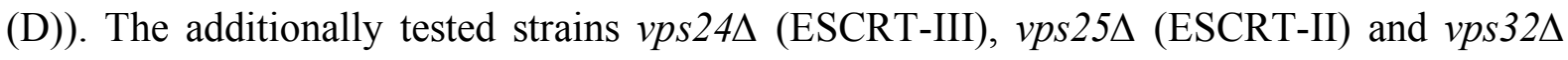
(ESCRT-III) also showed a drastical decrease of degraded mito-GFP compared to wild type cells (Figure $37(\mathrm{C})$ ). Quantification analysis supported these observations. In vps $24 \Delta$ cells a signal intensity of $21 \%$ for mitophagy compared to wild type cells was detected. Also vps $25 \Delta$ and vps32 cells showed a decrease in the mitophagic rate to $15 \%$ and $13 \%$ (Figure 37 (C)). As Vps23 is a member of ESCRT subunit 0 and Vps27 is the link between ESCRT-0 and ESCRT-I (Katzmann, 2003), the results of this study indicated, that proteins of ESCRT-I, -II and -III are needed for mitophagy, while ESCRT-0 is excluded. As the ESCRT subunit 0 and Vps27 are known to be responsible for the recognition of ubiquitinated cargo in MVB pathway (Katzmann, 2001; Bilodeau, 2003), such a role in mitophagy can be excluded.

Using the same conditions and a comparable plasmid for detection of mitophagy, another group has also identified $v p s 24 \Delta$ and $v p s 32 \Delta$ cells as ,partially defective“ as well as vps $2 \Delta$ cells, which also represent the ESCRT subunit III. In contrast to this study, vps25 $\Delta$ and vps $28 \Delta$ cells have not been defective in mitophagy (Okamoto, 2009).

Unselective autophagy was measured under the same growth conditions (72 hours in medium $2 \%$ lactate) using Pgk1-GFP in parallel. These strains showed only a slight defect concerning 
ESCRT -I, -II and -III (Table 19, chapter 4.4.6), indicating a special but not indispensable role in mitophagy of the tested ESCRT proteins.

So far, the role of the ESCRT machinery for mitophagy is unclear. Microautophagic degradation, including the selective degradation of mitochondria, has been suggested to be favored by cells grown on lactate medium (Kissová, 2007). As selective ESCRT subunits were required for mitophagy after growth on lactate medium, but not for unselective autophagy, this might point to a potential role of the ESCRT subunits in micromitophagy.

An inactivation of ESCRT subunits might cause a direct effect in a micromitophagydependent degradation of mitochondria, by preventing the closure of the membrane bud around mitochondrial structures. As the ESCRT machinery also affects vacuole biogenesis, an indirect effect caused by an impairment of the vacuolar membrane, can not be excluded.

\subsubsection{Ubiquitination affects mitophagy in S. cerevisiae}

A deletion of Doa4 is known to deplete the cellular ubiquitin level (Swaminathan, 1999; Ren, 2008). In S. cerevisiae, unselective autophagy was not affected in doa4t cells (Krick, 2010). In mammalian cells, the E3-like ubiquitin ligase Parkin was shown to play an irreplaceable role in designating mitochondria for mitophagy by ubiquitination (Narendra, 2008). Ubiquitination was further shown to affect mitochondrial morphology and inheritance.

Mitochondria continuously grow, divide and fuse throughout cellular life and it is the frequency of fission events relative to fusion events that largely determines steady-state organelle morphology (Shaw and Nunnari, 2002). Damaged parts of mitochondria remain fragmented and thus are believed to be preferentially sequestered by autophagosomes (Twig, 2008). Mitofusins are ubiquitinated in a PINK1- and Parkin-dependent manner in mammalian cells and Drosophila, suggesting a proteasomal degradation, which furthermore leads to a block in mitochondrial fusion machinery and an autophagy-dependent degradation of mitochondrial fragments (Gegg, 2010; Ziviani, 2010). Moreover, the ubiquitination of mammalian Drp1 affects mitochondrial fission (Chang and Blackstone).

A role of ubiquitin in labelling dysfunctional mitochondria might also be conceivable in $S$. cerevisiae. Therefore, the need of Doa4 for mitophagy compared to unselective autophagy was tested. Figure 38 (A) (chapter 4.4.7) shows a significant reduction in the mitophagic rate, while unselective autophagy was not strongly affected. These observations were supported by quantification analysis, which showed a significant decrease of mitophagy to $37 \%(\mathrm{SEM}=11)($ Figure $38(\mathrm{~B}))$. The decrease of unselective autophagy to $65 \%(\mathrm{SEM}=$ 
11) probably indicates a general effect on cellular health, caused by the depletion of the cellular ubiquitin level. The significant reduction in mitophagic degradation leads to speculations about a specific role of ubiquitin in mitophagy. Ubiquitin has seven lysines (lys), in which three are typically used for polyubiquitination. Notably, different ubiquitin chains have specific consequences for the tagged substrates and are linked to diverse cellular functions (Ikeda and Dikic, 2008). For instance, monoubiquitination or oligomeric Lys63linked ubiquitin chains target the proteins to the vacuole, whereas polyubiquitination linked via Lys48 of ubiquitin targets the proteins to the proteasome. In mammalian cells, Parkin has been shown to mediate the formation of two distinct polyubiquitin chains on the substrates at depolarised mitochondria, linked through Lys27 and Lys63 (Geisler, 2010). Hence, it has to be tested whether mono- or polyubiquitin is required for mitophagy in yeast. This can be done by mito-GFP degradation assays in doa4t cells using furthermore plasmids expressing different ubiquitin mutants, containing substitutions of defined lysines to arginines (e.g. Lys63 - Arg63).

Ubp3 and its cofactor Bre5 are needed for the starvation induced selective autophagic degradation of the large $60 \mathrm{~S}$ subunit of ribosomes (ribophagy), by removal of ubiquitin from a yet unknown substrate (Kraft, 2008). Nevertheless, experiments in this study showed that neither Ubp3 nor Bre5 impair the process of mitophagy (data not shown).

The need of Doa4 might give a hint for ubiquitination in labelling of dysfunctional mitochondria for degradation. As mitofusins are reported to be substrates for such a welldirected ubiquitination in mammalian cells (Gegg, 2010), also yeast's mitofusins might fulfill such a function.

The mitochondrial fusion machinery in $S$. cerevisiae is well understood. Fzo1, a mitochondrial outer membrane protein, is required for the fusion of mitochondria and therefore essential for the biogenesis of functional mitochondria. The depletion of this GTPase leads to a petite phenotype and fragmented mitochondrial morphology (Hermann, 1998; Rapaport, 1998). Another known dynamin-related GTPase is Mgm1 (Wong, 2000), which exists in two isoforms. The smaller isoform is generated by the cleaveage of Pcp1, a rhomboid-like serine protease. Even though the exact function of both isoforms is still not clear, the larger isoform was shown to be more important for mitochondrial fusion (Herlan, 2003). Furthermore Ugo1, a mitochondrial transmembrane protein located in the outer membrane, was shown to link Mgm1 to Fzol for mitochondrial fusion (Sesaki and Jensen, 2004). 
The dynamin-related GTPase Dnm1 participates in mitochondrial fission and therefore fulfills an antagonistic activity to Fzo1. Deletion of DNM1 leads to a block in membrane fission indicating a regulatory role (Bleazard, 1999), notably not resulting in a petite phenotype. Depending on the used conditions, $d n m 1 \Delta$ cells show slight to strong defects in mitophagy (Kanki, 2009b), indicating a role of the mitophagic fission machinery in mitophag. Alternatively, the autophagic core machinery could be recruited to damaged parts of the mitochondria and somehow promote fission to allow mitophagy.

Fzo1, Mgm1 and Ugo1, representing the mitochondrial fusion machinery, are good canditates for further investigations. As already mentioned, the deletion of FZO1 or also MGM1 lead to a petite phenotype, resulting in poor growth in medium containing lactate as the sole carbon source. The degradation assay of mito-GFP used in this study includes the preculturing in $2 \%$ lactate medium and is therefore not suitable. Preculturing of yeast in medium containing $2 \%$ glucose as carbon source leads to the so-called glucose repression, meaning the repression of genes required for metabolisms of alternative carbon sources, including respiratory enzymes (Strittmatter, 1957; Trumbly, 1992). Moreover, absence of mitochondrial structures was observed in yeast cells grown aerobically on glucose (Polakis, 1964). To avoid glucose repression, experiments were done using preculture medium containing $2 \%$ galactose as sole carbon source. First measurements using mito-GFP resulted in an undetectable signal for free GFP in wild type cells (data not shown), confirming observations of other groups (Kanki, 2009c; Okamoto, 2009). However, an optimisation of growth conditions using galactose as sole carbon source may lead to interesting results, elucidating the role of the membrane fusion machinery in mitophagy. This might also provide substrates for a proposed ubiquitination.

This study gives new insights into the selective degradation of mitochondria via mitophagy. The supposed role of Doa4 and some ESCRT proteins offers possibilities for a number of speculations.

A Vps27- and ESCRT subunit I-mediated recruitment of the ESCRT machinery to ubiquitinated mitochondria can be excluded. Atg32, which was shown to be essential for mitophagy in all experiments, might not require ubiquitination, due to its ability to bind Atg8 directly or by an interaction via Atg11 (Kanki, 2009c; Okamoto, 2009). An additional ubiquitination of Atg32 is therefore questionable. The suggested ubiquitination might be a signal for the cell to differentiate between superfluous or damaged, and therefore dysfunctional, mitochondria and might also be a trigger to induce micro- or macromitophagy. The possibility to distinguish between micro- and macromitophagy by different deletions is essential and therefore a major focus of further investigations. 


\subsection{The nucleolus might represent a specific cargo for PMN}

Since the discovery of PMN in 2003, this selective microautophagic degradation of parts of the nucleus, which requires a subset of autophagic proteins, became a focus of interest (Roberts, 2003; Krick, 2008b). Also Atg11, a protein required for cargo recruitment in the Cvt pathway or pexophagy (Kim, 2001), is required (Krick, 2008b). PMN is a unique microautophagic process at contact sites between the vacuole and the nucleus (NV junctions). These NV junctions are formed through specific interaction between Vac8 and the Cterminus of Nvj1, that is upregulated upon nutrient starvation. The deletion of Vac8 or Nvj1 blocks PMN. Nvj1 is furthermore believed to interact via its N-terminus with an unknown inner nuclear membrane protein (Kvam and Goldfarb, 2006). Tsc13, an ER-membrane protein, is sequestered into NV junctions from the ER by interacting with Nvj1 in a Vac8independent manner. As a consequence, it is incorporated into PMN structures and targeted to the vacuole (Kvam, 2005). Osh1 is a member of the Osh family in yeast, and a homologue of the mammalian oxysterol-binding protein (OSBP) (Levine and Munro, 2001), which may function in nonvesicular lipid trafficking. Osh1 is targeted to NV junctions through interaction with Nvj1, proportional to the expression of Nvj1, also in the absence of Vac8 (Kvam and Goldfarb, 2004). However, Osh1 is not necessary for the formation of the NV junctions or efficient PMN, but the depletion of the whole Osh family of yeast leads to a block in PMN, indicating overlapping functions of this protein family (Kvam and Goldfarb, 2004). Due to the interaction with NV junctions, Osh1 exhibits a substrate for PMN by incorporation into PMN structures and is therefore used as common marker protein (Kvam and Goldfarb, 2004; Millen, 2009).

Up to now, a specific nuclear cargo for PMN is still missing and is a major focus of investigation.

A specific cargo-recognition, indicated by the need of Atg11, is indispensable to preserve the integrity of the nucleus. Indeed chromosomal DNA and nuclear pore complexes are excluded from NV junctions (Pan, 2000; Roberts, 2003). As the TOR kinase arrests pre-rRNA processing and stabilizes pre-rRNA precursors in the nucleolus under starvation (Powers and Walter, 1999), Roberts et al. (2003) have speculated that preribosomes, which are no longer needed in the cytoplasm under nutrient limitation, might be substrates of PMN.

Western blot analysis of nucleolar GFP fusion proteins did not result in the identification of a selective PMN cargo (Figure 39, chapter 4.5.1), however the microscopic 
analysis of some nucleolus proteins under nutrient starvation after staining of the vacuolar membrane with FM4-64, resulted in interesting observations (Figure 40, chapter 4.5.2).

Nop8 is a nucleolar protein, required for 60S ribosomal subunit biogenesis (Zanchin and Goldfarb, 1999). Cells chromosomally expressing Nop8-GFP, showed a green fluorescent signal inside the vacuole, indicating the autophagy depending degradation of nucleolar material. Furthermore, cells expressing the nucleolar Cic1-GFP (Jager, 2001) seemed to have green vesicles inside the vacuole under nutrient starvation. In contrast Utp5-GFP and Utp10GFP, two small subunit proteins of the processome, which is a ribosome assembly intermediate (Bernstein, 2004), did not exhibit green fluorescent signal outside of the nucleolus. Notably, under nutrient starvation, in cells chromosomally expressing Sik1-GFP, nucleolar structures with a striking colocalisation with the vacuolar membrane were observed (Figure 40). For subsequent microscopy experiments, cells expressing Nog1-GFP were used. Nog1, a nuclear GTP-binding protein, is tethered to the nucleolus upon nutrient starvation or rapamycin treatment (Honma, 2006). This might indicate a translocation of Nog1-GFP to the PMN active side. Indeed, in starved Nog1-GFP cells, PMN-like, Nog1-GFP containing structures pinching off from the vacuolar membrane into the vacuole were observed (Figure 41, chapter 4.5.2).

Furthermore, in microscopic analysis of starved cells chromosomally expressing Sik1-RFP and plasmid encoded Osh1-GFP, NV junctions were shown to localise directly next to the nucleolus in about $70 \%$ of the cells (Figure 42 (A) and (B), chapter 4.5.3; SEM =0.6). For further investigations Sik1-RFP cells expressing the PAS marker GFP-Atg8 were used for microscopic analysis. In these cells, $30 \%$ of the observed PAS dots localised in close proximity to the nucleolus after starvation (Figure 43 (A) and (B), chapter 4.5.4). Notably, incubation of cells in SD-N medium does not selectively induce PMN.

Taken together, the experiments in this study point to a specific recognition of single nucleolar proteins. Especially the observation of GFP signal inside the vacuole of cells chromosomally expressing Nop8-GFP, might support the speculations of Roberts et al. (2003), suggesting preribosomes as a selective cargo for PMN by a TOR regulated pre-rRNA processing.

Nevertheless, a selective autophagic uptake of other nuclear proteins than the nucleolus components by PMN is not excluded.

Nevertheless, the detected GFP signals outside the nucleolus in the tested strains appeared weak and no clear free-floating autophagic bodies inside the vacuole were observed under PMSF treatment. Additionally, western blot analysis showed no degradation of the 
nucleolar fusion proteins, maybe because of the slight amount that was degraded by PMN. Microscopic analysis of further nucleolar or nuclear proteins, also by time-lapse experiments, might identify a specific nucleolar or nuclear cargo for PMN. 


\section{Literature}

Amerik, A.Y., Nowak, J., Swaminathan, S.und Hochstrasser, M. (2000). The Doa4 deubiquitinating enzyme is functionally linked to the vacuolar protein-sorting and endocytic pathways. Mol Biol Cell 11, 3365-3380.

Baba, M., Osumi, M., Scott, S.V., Klionsky, D.J.und Ohsumi, Y. (1997). Two distinct pathways for targeting proteins from the cytoplasm to the vacuole/lysosome. The Journal of Cell Biology 139, 1687-1695.

Baba, M., Takeshige, K., Baba, N.und Ohsumi, Y. (1994). Ultrastructural analysis of the autophagic process in yeast: detection of autophagosomes and their characterization. The Journal of Cell Biology 124, 903-913.

Babst, M. (2005). A protein's final ESCRT. Traffic 6, 2-9.

Babst, M., Katzmann, D.J., Estepa-Sabal, E.J., Meerloo, T.und Emr, S.D. (2002). Escrt-III: an endosome-associated heterooligomeric protein complex required for mvb sorting. Dev Cell 3, 271-282.

Babst, M., Sato, T.K., Banta, L.M.und Emr, S.D. (1997). Endosomal transport function in yeast requires a novel AAA-type ATPase, Vps4p. EMBO J 16, 1820-1831.

Barth, H., Meiling-Wesse, K., Epple, U.D.und Thumm, M. (2001). Autophagy and the cytoplasm to vacuole targeting pathway both require Aut10p. FEBS Letters 508, 23-28.

Barth, H., Meiling-Wesse, K., Epple, U.D.und Thumm, M. (2002). Mailp is essential for maturation of proaminopeptidase I but not for autophagy. FEBS Letters 512, 173-179.

Baxter, B.K., Abeliovich, H., Zhang, X., Stirling, A.G., Burlingame, A.L.und Goldfarb, D.S. (2005). Atg19p ubiquitination and the cytoplasm to vacuole trafficking pathway in yeast. $\mathrm{J}$ Biol Chem 280, 39067-39076.

Bernstein, K.A., Gallagher, J.E., Mitchell, B.M., Granneman, S.und Baserga, S.J. (2004). The small-subunit processome is a ribosome assembly intermediate. Eukaryot Cell 3, 1619-1626.

Bilodeau, P.S., Winistorfer, S.C., Kearney, W.R., Robertson, A.D.und Piper, R.C. (2003). Vps27-Hse1 and ESCRT-I complexes cooperate to increase efficiency of sorting ubiquitinated proteins at the endosome. J Cell Biol 163, 237-243.

Bleazard, W., McCaffery, J.M., King, E.J., Bale, S., Mozdy, A., Tieu, Q., Nunnari, J.und Shaw, J.M. (1999). The dynamin-related GTPase Dnm1 regulates mitochondrial fission in yeast. Nat Cell Biol 1, 298-304.

Bogomolnaya, L.M., Pathak, R., Cham, R., Guo, J., Surovtseva, Y.V., Jaeckel, L.und Polymenis, M. (2004). A new enrichment approach identifies genes that alter cell cycle progression in Saccharomyces cerevisiae. Curr Genet 45, 350-359. 
Braun, R.und Zischka, H. (2008). Mechanisms of Cdc48/VCP-mediated cell death - from yeast apoptosis to human disease. Biochimica et Biophysica Acta (BBA) - Molecular Cell Research 1783, 1418-1435.

Braun, S., Matuschewski, K., Rape, M., Thoms, S.und Jentsch, S. (2002). Role of the ubiquitin-selective CDC48(UFD1/NPL4 )chaperone (segregase) in ERAD of OLE1 and other substrates. EMBO J 21, 615-621.

Bremer, S. (2009). Molecular analysis of vesicle biogenesis during autophagy. Dissertation, Georg August Universität, Göttingen.

Brody, J.R.und Kern, S.E. (2004). History and principles of conductive media for standard DNA electrophoresis. Anal Biochem 333, 1-13.

Bryant, N.J.und Stevens, T.H. (1998). Vacuole biogenesis in Saccharomyces cerevisiae: protein transport pathways to the yeast vacuole. Microbiol Mol Biol Rev 62, 230-247.

Buchberger, A. (2010). Control of ubiquitin conjugation by cdc48 and its cofactors. Subcell Biochem 54, 17-30.

Buchberger, A., Howard, M.J., Proctor, M.und Bycroft, M. (2001). The UBX domain: a widespread ubiquitin-like module. J Mol Biol 307, 17-24.

Burd, C.G.und Emr, S.D. (1998). Phosphatidylinositol(3)-phosphate signaling mediated by specific binding to RING FYVE domains. Mol Cell 2, 157-162.

Campbell, C.L.und Thorsness, P.E. (1998). Escape of mitochondrial DNA to the nucleus in yme1 yeast is mediated by vacuolar-dependent turnover of abnormal mitochondrial compartments. J Cell Sci 111 ( Pt 16), 2455-2464.

Chang, C.R.und Blackstone, C. Dynamic regulation of mitochondrial fission through modification of the dynamin-related protein Drp1. Ann N Y Acad Sci 1201, 34-39.

Chen, Y., Azad, M.B.und Gibson, S.B. (2009). Superoxide is the major reactive oxygen species regulating autophagy. Cell Death Differ 16, 1040-1052.

Cocheme, H.M.und Murphy, M.P. (2008). Complex I is the major site of mitochondrial superoxide production by paraquat. J Biol Chem 283, 1786-1798.

Collinson, L.P.und Dawes, I.W. (1992). Inducibility of the response of yeast cells to peroxide stress. J Gen Microbiol 138, 329-335.

Conibear, E.und Stevens, T.H. (1998). Multiple sorting pathways between the late Golgi and the vacuole in yeast. Biochim Biophys Acta 1404, 211-230.

Cuervo, A.M. (2010). Chaperone-mediated autophagy: selectivity pays off. Trends Endocrinol Metab 21, 142-150.

Darsow, T., Rieder, S.E.und Emr, S.D. (1997). A multispecificity syntaxin homologue, Vam3p, essential for autophagic and biosynthetic protein transport to the vacuole. J Cell Biol $138,517-529$. 
Dove, S.K., Piper, R.C., McEwen, R.K., Yu, J.W., King, M.C., Hughes, D.C., Thuring, J., Holmes, A.B., Cooke, F.T., Michell, R.H., Parker, P.J.und Lemmon, M.A. (2004). Svplp defines a family of phosphatidylinositol 3,5-bisphosphate effectors. EMBO J 23, 1922-1933.

Dragon, F., Gallagher, J.E., Compagnone-Post, P.A., Mitchell, B.M., Porwancher, K.A., Wehner, K.A., Wormsley, S., Settlage, R.E., Shabanowitz, J., Osheim, Y., Beyer, A.L., Hunt, D.F.und Baserga, S.J. (2002). A large nucleolar U3 ribonucleoprotein required for $18 \mathrm{~S}$ ribosomal RNA biogenesis. Nature 417, 967-970.

Epple, U.D., Eskelinen, E.L.und Thumm, M. (2003). Intravacuolar membrane lysis in Saccharomyces cerevisiae. Does vacuolar targeting of Cvt17/Aut5p affect its function? J Biol Chem 278, 7810-7821.

Epple, U.D., Suriapranata, I., Eskelinen, E.L.und Thumm, M. (2001). Aut5/Cvt17p, a putative lipase essential for disintegration of autophagic bodies inside the vacuole. J Bacteriol 183, 5942-5955.

Eskelinen, E.L. (2005). Maturation of autophagic vacuoles in Mammalian cells. Autophagy 1, $1-10$.

Fader, C.M.und Colombo, M.I. (2009). Autophagy and multivesicular bodies: two closely related partners. Cell Death Differ 16, 70-78.

Farre, J.C., Krick, R., Subramani, S.und Thumm, M. (2009). Turnover of organelles by autophagy in yeast. Curr Opin Cell Biol 21, 522-530.

Farré, J.C., Manjithaya, R., Mathewson, R.D.und Subramani, S. (2008). PpAtg30 tags peroxisomes for turnover by selective autophagy. Dev Cell 14, 365-376.

Filimonenko, M., Stuffers, S., Raiborg, C., Yamamoto, A., Malerod, L., Fisher, E.M., Isaacs, A., Brech, A., Stenmark, H.und Simonsen, A. (2007). Functional multivesicular bodies are required for autophagic clearance of protein aggregates associated with neurodegenerative disease. J Cell Biol 179, 485-500.

Fischer von Mollard, G.und Stevens, T.H. (1999). The Saccharomyces cerevisiae v-SNARE Vtilp is required for multiple membrane transport pathways to the vacuole. Mol Biol Cell 10, 1719-1732.

Gegg, M.E., Cooper, J.M., Chau, K.Y., Rojo, M., Schapira, A.H.und Taanman, J.W. (2010). Mitofusin 1 and mitofusin 2 are ubiquitinated in a PINK1/parkin-dependent manner upon induction of mitophagy. Hum Mol Genet 19, 4861-4870.

Geisler, S., Holmstrom, K.M., Skujat, D., Fiesel, F.C., Rothfuss, O.C., Kahle, P.J.und Springer, W. (2010). PINK1/Parkin-mediated mitophagy is dependent on VDAC1 and p62/SQSTM1. Nat Cell Biol 12, 119-131.

Goldman, S.J., Taylor, R., Zhang, Y.und Jin, S. (2010). Autophagy and the degradation of mitochondria. Mitochondrion 10,309-315. 
Guan, J., Stromhaug, P.E., George, M.D., Habibzadegah-Tari, P., Bevan, A., Dunn, W.A.und Klionsky, D.J. (2001). Cvt18/Gsa12 is required for cytoplasm-to-vacuole transport, pexophagy, and autophagy in Saccharomyces cerevisiae and Pichia pastoris. Mol Biol Cell $12,3821-3838$.

Hanahan, D. (1983). Studies on transformation of Escherichia coli with plasmids. J Mol Biol $166,557-580$.

Hanahan, D., Jessee, J.und Bloom, F.R. (1991). Plasmid transformation of Escherichia coli and other bacteria. Methods Enzymol 204, 63-113.

Harding, T.M., Hefner-Gravink, A., Thumm, M.und Klionsky, D.J. (1996). Genetic and phenotypic overlap between autophagy and the cytoplasm to vacuole protein targeting pathway. J Biol Chem 271, 17621-17624.

Hartmann-Petersen, R., Wallace, M., Hofmann, K., Koch, G., Johnsen, A.H., Hendil, K.B.und Gordon, C. (2004). The Ubx2 and Ubx3 cofactors direct Cdc48 activity to proteolytic and nonproteolytic ubiquitin-dependent processes. Curr Biol 14, 824-828.

Hassan, H.M.und Fridovich, I. (1979). Intracellular production of superoxide radical and of hydrogen peroxide by redox active compounds. Arch Biochem Biophys 196, 385-395.

He, C., Song, H., Yorimitsu, T., Monastyrska, I., Yen, W.L., Legakis, J.E.und Klionsky, D.J. (2006). Recruitment of Atg9 to the preautophagosomal structure by Atg11 is essential for selective autophagy in budding yeast. The Journal of Cell Biology 175, 925-935.

Herlan, M., Vogel, F., Bornhovd, C., Neupert, W.und Reichert, A.S. (2003). Processing of Mgm1 by the rhomboid-type protease Pcp1 is required for maintenance of mitochondrial morphology and of mitochondrial DNA. J Biol Chem 278, 27781-27788.

Hermann, G.J., Thatcher, J.W., Mills, J.P., Hales, K.G., Fuller, M.T., Nunnari, J.und Shaw, J.M. (1998). Mitochondrial fusion in yeast requires the transmembrane GTPase Fzolp. J Cell Biol 143, 359-373.

Herskowitz, I. (1988). Life cycle of the budding yeast Saccharomyces cerevisiae. Microbiol Rev 52, 536-553.

Hoffmann, W. (1985). Molecular characterization of the CAN1 locus in Saccharomyces cerevisiae. A transmembrane protein without $\mathrm{N}$-terminal hydrophobic signal sequence. J Biol Chem 260, 11831-11837.

Honma, Y., Kitamura, A., Shioda, R., Maruyama, H., Ozaki, K., Oda, Y., Mini, T., Jeno, P., Maki, Y., Yonezawa, K., Hurt, E., Ueno, M., Uritani, M., Hall, M.N.und Ushimaru, T. (2006). TOR regulates late steps of ribosome maturation in the nucleoplasm via Nog1 in response to nutrients. EMBO J 25, 3832-3842.

Horvath, A.und Riezman, H. (1994). Rapid protein extraction from Saccharomyces cerevisiae. Yeast 10, 1305-1310. 
Huh, W.K., Falvo, J.V., Gerke, L.C., Carroll, A.S., Howson, R.W., Weissman, J.S.und O'Shea, E.K. (2003). Global analysis of protein localization in budding yeast. Nature 425, 686-691.

Hurley, J.H.und Emr, S.D. (2006). The ESCRT complexes: structure and mechanism of a membrane-trafficking network. Annual review of biophysics and biomolecular structure 35, 277-298.

Hurley, J.H.und Hanson, P.I. (2010). Membrane budding and scission by the ESCRT machinery: it's all in the neck. Nat Rev Mol Cell Biol 11, 556-566.

Hutchins, M.U., Veenhuis, M.und Klionsky, D.J. (1999). Peroxisome degradation in Saccharomyces cerevisiae is dependent on machinery of macroautophagy and the Cvt pathway. J Cell Sci 112 ( Pt 22), 4079-4087.

Ichimura, Y., Kirisako, T., Takao, T., Satomi, Y., Shimonishi, Y., Ishihara, N., Mizushima, N., Tanida, I., Kominami, E., Ohsumi, M., Noda, T.und Ohsumi, Y. (2000). A ubiquitin-like system mediates protein lipidation. Nature 408, 488-492.

Ikeda, F.und Dikic, I. (2008). Atypical ubiquitin chains: new molecular signals. 'Protein Modifications: Beyond the Usual Suspects' review series. EMBO Rep 9, 536-542.

Inoue, Y.und Klionsky, D.J. (2010). Regulation of macroautophagy in Saccharomyces cerevisiae. Semin Cell Dev Biol 21, 664-670.

Ishihara, N., Hamasaki, M., Yokota, S., Suzuki, K., Kamada, Y., Kihara, A., Yoshimori, T., Noda, T.und Ohsumi, Y. (2001). Autophagosome requires specific early Sec proteins for its formation and NSF/SNARE for vacuolar fusion. Mol Biol Cell 12, 3690-3702.

Jaeger, P.A.und Wyss-Coray, T. (2010). Beclin 1 complex in autophagy and Alzheimer disease. Arch Neurol 67, 1181-1184.

Jager, S., Strayle, J., Heinemeyer, W.und Wolf, D.H. (2001). Cic1, an adaptor protein specifically linking the $26 \mathrm{~S}$ proteasome to its substrate, the SCF component Cdc4. EMBO J $20,4423-4431$.

Jamieson, D.J. (1998). Oxidative stress responses of the yeast Saccharomyces cerevisiae. Yeast 14, 1511-1527.

Janke, C., Magiera, M.M., Rathfelder, N., Taxis, C., Reber, S., Maekawa, H., MorenoBorchart, A., Doenges, G., Schwob, E., Schiebel, E.und Knop, M. (2004). A versatile toolbox for PCR-based tagging of yeast genes: new fluorescent proteins, more markers and promoter substitution cassettes. Yeast 21, 947-962.

Jarosch, E., Taxis, C., Volkwein, C., Bordallo, J., Finley, D., Wolf, D.H.und Sommer, T. (2002). Protein dislocation from the ER requires polyubiquitination and the AAA-ATPase Cdc48. Nat Cell Biol 4, 134-139.

Kabeya, Y., Kamada, Y., Baba, M., Takikawa, H., Sasaki, M.und Ohsumi, Y. (2005). Atg17 functions in cooperation with Atg1 and Atg13 in yeast autophagy. Mol Biol Cell 16, 25442553. 
Kamada, Y., Funakoshi, T., Shintani, T., Nagano, K., Ohsumi, M.und Ohsumi, Y. (2000). Tor-mediated induction of autophagy via an Apg1 protein kinase complex. The Journal of Cell Biology 150, 1507-1513.

Kanki, T., Kang, D.und Klionsky, D.J. (2009a). Monitoring mitophagy in yeast: the Om45GFP processing assay. Autophagy 5, 1186-1189.

Kanki, T.und Klionsky, D.J. (2008). Mitophagy in yeast occurs through a selective mechanism. J Biol Chem 283, 32386-32393.

Kanki, T.und Klionsky, D.J. (2009). Atg32 is a tag for mitochondria degradation in yeast. Autophagy 5, 1201-1202.

Kanki, T., Wang, K., Baba, M., Bartholomew, C.R., Lynch-Day, M.A., Du, Z., Geng, J., Mao, K., Yang, Z., Yen, W.L.und Klionsky, D.J. (2009b). A genomic screen for yeast mutants defective in selective mitochondria autophagy. Mol Biol Cell 20, 4730-4738.

Kanki, T., Wang, K., Cao, Y., Baba, M.und Klionsky, D.J. (2009c). Atg32 is a mitochondrial protein that confers selectivity during mitophagy. Dev Cell 17, 98-109.

Katzmann, D.J., Babst, M.und Emr, S.D. (2001). Ubiquitin-dependent sorting into the multivesicular body pathway requires the function of a conserved endosomal protein sorting complex, ESCRT-I. Cell 106, 145-155.

Katzmann, D.J., Stefan, C.J., Babst, M.und Emr, S.D. (2003). Vps27 recruits ESCRT machinery to endosomes during MVB sorting. J Cell Biol 162, 413-423.

Kawamata, T., Kamada, Y., Kabeya, Y., Sekito, T.und Ohsumi, Y. (2008). Organization of the pre-autophagosomal structure responsible for autophagosome formation. Mol Biol Cell 19, 2039-2050.

Keeble, J.A.und Gilmore, A.P. (2007). Apoptosis commitment--translating survival signals into decisions on mitochondria. Cell Res 17, 976-984.

Kiffin, R., Bandyopadhyay, U.und Cuervo, A.M. (2006). Oxidative stress and autophagy. Antioxid Redox Signal 8, 152-162.

Kihara, A., Noda, T., Ishihara, N.und Ohsumi, Y. (2001). Two distinct Vps34 phosphatidylinositol 3-kinase complexes function in autophagy and carboxypeptidase $\mathrm{Y}$ sorting in Saccharomyces cerevisiae. The Journal of Cell Biology 152, 519-530.

Kim, J., Huang, W.P., Stromhaug, P.E.und Klionsky, D.J. (2002). Convergence of multiple autophagy and cytoplasm to vacuole targeting components to a perivacuolar membrane compartment prior to de novo vesicle formation. J Biol Chem 277, 763-773.

Kim, J., Kamada, Y., Stromhaug, P.E., Guan, J., Hefner-Gravink, A., Baba, M., Scott, S.V., Ohsumi, Y., Dunn, W.A., Jr.und Klionsky, D.J. (2001). Cvt9/Gsa9 functions in sequestering selective cytosolic cargo destined for the vacuole. J Cell Biol 153, 381-396. 
Kirisako, T., Baba, M., Ishihara, N., Miyazawa, K., Ohsumi, M., Yoshimori, T., Noda, T.und Ohsumi, Y. (1999). Formation process of autophagosome is traced with Apg8/Aut7p in yeast. The Journal of Cell Biology 147, 435-446.

Kirisako, T., Ichimura, Y., Okada, H., Kabeya, Y., Mizushima, N., Yoshimori, T., Ohsumi, M., Takao, T., Noda, T.und Ohsumi, Y. (2000). The reversible modification regulates the membrane-binding state of Apg8/Aut7 essential for autophagy and the cytoplasm to vacuole targeting pathway. The Journal of Cell Biology 151, 263-276.

Kissová, I., Deffieu, M., Manon, S.und Camougrand, N. (2004). Uth1p is involved in the autophagic degradation of mitochondria. J Biol Chem 279, 39068-39074.

Kissová, I., Salin, B., Schaeffer, J., Bhatia, S., Manon, S.und Camougrand, N. (2007). Selective and non-selective autophagic degradation of mitochondria in yeast. Autophagy 3 , 329-336.

Klionsky, D.J. (2005). Autophagy. Curr Biol 15, R282-283.

Klionsky, D.J., Cregg, J.M., Dunn, W.A., Emr, S.D., Sakai, Y., Sandoval, I.V., Sibirny, A., Subramani, S., Thumm, M., Veenhuis, M.und Ohsumi, Y. (2003). A unified nomenclature for yeast autophagy-related genes. Dev Cell 5, 539-545.

Klionsky, D.J., Cuervo, A.M.und Seglen, P.O. (2007). Methods for monitoring autophagy from yeast to human. Autophagy 3, 181-206.

Klionsky, D.J., Cueva, R.und Yaver, D.S. (1992). Aminopeptidase I of Saccharomyces cerevisiae is localized to the vacuole independent of the secretory pathway. J Cell Biol 119, 287-299.

Kraft, C., Deplazes, A., Sohrmann, M.und Peter, M. (2008). Mature ribosomes are selectively degraded upon starvation by an autophagy pathway requiring the Ubp3p/Bre $5 p$ ubiquitin protease. Nat Cell Biol 10, 602-610.

Krick, R., Bremer, S., Welter, E., Schlotterhose, P., Muehe, Y., Eskelinen, E.L.und Thumm, M. (2010). Cdc48/p97 and Shp1/p47 regulate autophagosome biogenesis in concert with ubiquitin-like Atg8. J Cell Biol 190, 965-973.

Krick, R., Henke, S., Tolstrup, J.und Thumm, M. (2008a). Dissecting the localization and function of Atg18, Atg21 and Ygr223c. Autophagy 4, 896-910.

Krick, R., Muehe, Y., Prick, T., Bremer, S., Schlotterhose, P., Eskelinen, E.L., Millen, J., Goldfarb, D.S.und Thumm, M. (2008b). Piecemeal microautophagy of the nucleus requires the core macroautophagy genes. Mol Biol Cell 19, 4492-4505.

Krick, R., Mühe, Y., Prick, T., Bredschneider, M., Bremer, S., Wenzel, D., Eskelinen, E.L.und Thumm, M. (2009). Piecemeal microautophagy of the nucleus: Genetic and morphological traits. Autophagy 5.

Krick, R., Tolstrup, J., Appelles, A., Henke, S.und Thumm, M. (2006). The relevance of the phosphatidylinositolphosphat-binding motif FRRGT of Atg18 and Atg21 for the Cvt pathway and autophagy. FEBS Letters 580, 4632-4638. 
Kroemer, G., Galluzzi, L.und Brenner, C. (2007). Mitochondrial membrane permeabilization in cell death. Physiol Rev 87, 99-163.

Kvam, E., Gable, K., Dunn, T.M.und Goldfarb, D. (2005). Targeting of Tsc13p to nucleusvacuole junctions: a role for very-long-chain fatty acids in the biogenesis of microautophagic vesicles. Mol Biol Cell 16, 3987-3998.

Kvam, E.und Goldfarb, D. (2004). Nvj1p is the outer-nuclear-membrane receptor for oxysterol-binding protein homolog Osh1p in Saccharomyces cerevisiae. Journal of Cell Science 117, 4959-4968.

Kvam, E.und Goldfarb, D. (2006). Structure and function of nucleus-vacuole junctions: outernuclear-membrane targeting of Nvj1p and a role in tryptophan uptake. Journal of Cell Science $119,3622-3633$.

Laemmli, U.K. (1970). Cleavage of structural proteins during the assembly of the head of bacteriophage T4. Nature 227, 680-685.

Latterich, M., Fröhlich, K.U.und Schekman, R. (1995). Membrane fusion and the cell cycle: Cdc48p participates in the fusion of ER membranes. Cell 82, 885-893.

Lee, F.J.und Hassan, H.M. (1985). Biosynthesis of superoxide dismutase in Saccharomyces cerevisiae: effects of paraquat and copper. J Free Radic Biol Med 1, 319-325.

Leonhard, K., Herrmann, J.M., Stuart, R.A., Mannhaupt, G., Neupert, W.und Langer, T. (1996). AAA proteases with catalytic sites on opposite membrane surfaces comprise a proteolytic system for the ATP-dependent degradation of inner membrane proteins in mitochondria. EMBO J 15, 4218-4229.

Levine, T.P.und Munro, S. (2001). Dual targeting of Osh1p, a yeast homologue of oxysterolbinding protein, to both the Golgi and the nucleus-vacuole junction. Mol Biol Cell 12, 16331644.

Loewen, C.J., Roy, A.und Levine, T.P. (2003). A conserved ER targeting motif in three families of lipid binding proteins and in Opilp binds VAP. EMBO J 22, 2025-2035.

Longatti, A.und Tooze, S.A. (2009). Vesicular trafficking and autophagosome formation. Cell Death Differ 16, 956-965.

Longtine, M.S., McKenzie, A., Demarini, D.J., Shah, N.G., Wach, A., Brachat, A., Philippsen, P.und Pringle, J.R. (1998). Additional modules for versatile and economical PCRbased gene deletion and modification in Saccharomyces cerevisiae. Yeast 14, 953-961.

Mari, M., Griffith, J., Rieter, E., Krishnappa, L., Klionsky, D.J.und Reggiori, F. (2010). An Atg9-containing compartment that functions in the early steps of autophagosome biogenesis. J Cell Biol 190, 1005-1022.

Matsuda, N., Sato, S., Shiba, K., Okatsu, K., Saisho, K., Gautier, C.A., Sou, Y.S., Saiki, S., Kawajiri, S., Sato, F., Kimura, M., Komatsu, M., Hattori, N.und Tanaka, K. (2010). PINK1 
stabilized by mitochondrial depolarization recruits Parkin to damaged mitochondria and activates latent Parkin for mitophagy. J Cell Biol 189, 211-221.

Meiling-Wesse, K., Barth, H.und Thumm, M. (2002). Ccz1p/Aut11p/Cvt16p is essential for autophagy and the cvt pathway. FEBS Lett 526, 71-76.

Meiling-Wesse, K., Barth, H., Voss, C., Eskelinen, E.L., Epple, U.D.und Thumm, M. (2004). Atg21 is required for effective recruitment of Atg8 to the preautophagosomal structure during the Cvt pathway. J Biol Chem 279, 37741-37750.

Mewes, H.W., Albermann, K., Bahr, M., Frishman, D., Gleissner, A., Hani, J., Heumann, K., Kleine, K., Maierl, A., Oliver, S.G., Pfeiffer, F.und Zollner, A. (1997). Overview of the yeast genome. Nature 387, 7-65.

Meyer, H.H. (2005). Golgi reassembly after mitosis:

The AAA family meets the ubiquitin family. Biochimica et Biophysica Acta (BBA) Molecular Cell Research 1744, 465-517.

Meyer, H.H., Kondo, H.und Warren, G. (1998). The p47 co-factor regulates the ATPase activity of the membrane fusion protein, p97. FEBS Lett 437, 255-257.

Meyer, H.H., Shorter, J.G., Seemann, J., Pappin, D.und Warren, G. (2000). A complex of mammalian ufd1 and npl4 links the AAA-ATPase, p97, to ubiquitin and nuclear transport pathways. EMBO J 19, 2181-2192.

Meyer, H.H., Wang, Y.und Warren, G. (2002). Direct binding of ubiquitin conjugates by the mammalian p97 adaptor complexes, p47 and Ufd1-Np14. EMBO J 21, 5645-5652.

Millen, J., Krick, R., Prick, T., Thumm, M.und Goldfarb, D. (2009). Measuring piecemeal microautophagy of the nucleus in Saccharomyces cerevisiae. Autophagy 5, 75-81.

Misra, S.und Hurley, J.H. (1999). Crystal structure of a phosphatidylinositol 3-phosphatespecific membrane-targeting motif, the FYVE domain of Vps27p. Cell 97, 657-666.

Mizuno, Y., Ohta, S., Tanaka, M., Takamiya, S., Suzuki, K., Sato, T., Oya, H., Ozawa, T.und Kagawa, Y. (1989). Deficiencies in complex I subunits of the respiratory chain in Parkinson's disease. Biochem Biophys Res Commun 163, 1450-1455.

Nakatogawa, H., Ichimura, Y.und Ohsumi, Y. (2007). Atg8, a ubiquitin-like protein required for autophagosome formation, mediates membrane tethering and hemifusion. Cell 130, 165178.

Nakatogawa, H., Suzuki, K., Kamada, Y.und Ohsumi, Y. (2009). Dynamics and diversity in autophagy mechanisms: lessons from yeast. Nat Rev Mol Cell Biol 10, 458-467.

Narendra, D., Tanaka, A., Suen, D.F.und Youle, R.J. (2008). Parkin is recruited selectively to impaired mitochondria and promotes their autophagy. J Cell Biol 183, 795-803.

Narendra, D.P., Jin, S.M., Tanaka, A., Suen, D.F., Gautier, C.A., Shen, J., Cookson, M.R.und Youle, R.J. (2010a). PINK1 is selectively stabilized on impaired mitochondria to activate Parkin. PLoS Biol 8, e1000298. 
Narendra, D.P., Kane, L.A., Hauser, D.N., Fearnley, I.M.und Youle, R.J. (2010b). p62/SQSTM1 is required for Parkin-induced mitochondrial clustering but not mitophagy; VDAC1 is dispensable for both. Autophagy 6.

Nieminen, A.L. (2003). Apoptosis and necrosis in health and disease: role of mitochondria. Int Rev Cytol 224, 29-55.

Nixon, R.A. (2007). Autophagy, amyloidogenesis and Alzheimer disease. J Cell Sci 120, 4081-4091.

Noda, T., Matsuura, A., Wada, Y.und Ohsumi, Y. (1995). Novel system for monitoring autophagy in the yeast Saccharomyces cerevisiae. Biochem Biophys Res Commun 210, 126132.

Noda, T.und Ohsumi, Y. (1998). Tor, a phosphatidylinositol kinase homologue, controls autophagy in yeast. J Biol Chem 273, 3963-3966.

Ohsumi, Y.und Mizushima, N. (2004). Two ubiquitin-like conjugation systems essential for autophagy. Semin Cell Dev Biol 15, 231-236.

Okamoto, K., Kondo-Okamoto, N.und Ohsumi, Y. (2009). Mitochondria-anchored receptor Atg32 mediates degradation of mitochondria via selective autophagy. Dev Cell 17, 87-97.

Palermo, V., Falcone, C.und Mazzoni, C. (2007). Apoptosis and aging in mitochondrial morphology mutants of S. cerevisiae. Folia Microbiol (Praha) 52, 479-483.

Pan, X., Roberts, P., Chen, Y., Kvam, E., Shulga, N., Huang, K., Lemmon, S.und Goldfarb, D.S. (2000). Nucleus-vacuole junctions in Saccharomyces cerevisiae are formed through the direct interaction of Vac8p with Nvj1p. Mol Biol Cell 11, 2445-2457.

Pankiv, S., Clausen, T.H., Lamark, T., Brech, A., Bruun, J.A., Outzen, H., Overvatn, A., Bjorkoy, G.und Johansen, T. (2007). p62/SQSTM1 binds directly to Atg8/LC3 to facilitate degradation of ubiquitinated protein aggregates by autophagy. J Biol Chem 282, 2413124145.

Peterson, M.R., Burd, C.G.und Emr, S.D. (1999). Vaclp coordinates Rab and phosphatidylinositol 3-kinase signaling in Vps45p-dependent vesicle docking/fusion at the endosome. Curr Biol 9, 159-162.

Piper, R.C., Cooper, A.A., Yang, H.und Stevens, T.H. (1995). VPS27 controls vacuolar and endocytic traffic through a prevacuolar compartment in Saccharomyces cerevisiae. The Journal of Cell Biology 131, 603-617.

Polakis, E.S., Bartley, W.und Meek, G.A. (1964). Changes in the structure and enzyme activity of Saccharomyces cerevisiae in response to changes in the environment. Biochem $\mathrm{J}$ 90, 369-374.

Powers, T.und Walter, P. (1999). Regulation of ribosome biogenesis by the rapamycinsensitive TOR-signaling pathway in Saccharomyces cerevisiae. Mol Biol Cell 10, 987-1000. 
Prick, T.und Thumm, M. (2008). Measuring macroautophagy in S. cerevisiae: autophagic body accumulation and total protein turnover. Methods Enzymol 451, 57-66.

Raiborg, C.und Stenmark, H. (2009). The ESCRT machinery in endosomal sorting of ubiquitylated membrane proteins. Nature 458, 445-452.

Rapaport, D., Brunner, M., Neupert, W.und Westermann, B. (1998). Fzolp is a mitochondrial outer membrane protein essential for the biogenesis of functional mitochondria in Saccharomyces cerevisiae. J Biol Chem 273, 20150-20155.

Reggiori, F.und Klionsky, D.J. (2002). Autophagy in the eukaryotic cell. Eukaryot Cell 1, 1121.

Reggiori, F., Shintani, T., Nair, U.und Klionsky, D.J. (2005). Atg9 cycles between mitochondria and the pre-autophagosomal structure in yeasts. Autophagy 1, 101-109.

Reggiori, F., Tucker, K.A., Stromhaug, P.E.und Klionsky, D.J. (2004a). The Atg1-Atg13 complex regulates Atg9 and Atg23 retrieval transport from the pre-autophagosomal structure. Dev Cell 6, 79-90.

Reggiori, F., Wang, C.W., Nair, U., Shintani, T., Abeliovich, H.und Klionsky, D.J. (2004b). Early stages of the secretory pathway, but not endosomes, are required for Cvt vesicle and autophagosome assembly in Saccharomyces cerevisiae. Mol Biol Cell 15, 2189-2204.

Ren, J., Pashkova, N., Winistorfer, S.und Piper, R. (2008). DOA1/UFD3 Plays a Role in Sorting Ubiquitinated Membrane Proteins into Multivesicular Bodies. Journal of Biological Chemistry 283, 21599-21611.

Renart, J., Reiser, J.und Stark, G.R. (1979). Transfer of proteins from gels to diazobenzyloxymethyl-paper and detection with antisera: a method for studying antibody specificity and antigen structure. Proc Natl Acad Sci U S A 76, 3116-3120.

Rieder, S.E., Banta, L.M., Kohrer, K., McCaffery, J.M.und Emr, S.D. (1996). Multilamellar endosome-like compartment accumulates in the yeast vps28 vacuolar protein sorting mutant. Mol Biol Cell 7, 985-999.

Roberts, P., Moshitch-Moshkovitz, S., Kvam, E., O'Toole, E., Winey, M.und Goldfarb, D. (2003). Piecemeal microautophagy of nucleus in Saccharomyces cerevisiae. Mol Biol Cell 14, 129-141.

Rusten, T.E.und Stenmark, H. (2009). How do ESCRT proteins control autophagy? J Cell Sci 122, 2179-2183.

Saiki, R.K., Gelfand, D.H., Stoffel, S., Scharf, S.J., Higuchi, R., Horn, G.T., Mullis, K.B.und Erlich, H.A. (1988). Primer-directed enzymatic amplification of DNA with a thermostable DNA polymerase. Science 239, 487-491.

Sakai, Y., Oku, M., van der Klei, I.J.und Kiel, J.A. (2006). Pexophagy: autophagic degradation of peroxisomes. Biochim Biophys Acta 1763, 1767-1775. 
Sato, T.K., Darsow, T.und Emr, S.D. (1998). Vam7p, a SNAP-25-like molecule, and Vam3p, a syntaxin homolog, function together in yeast vacuolar protein trafficking. Mol Cell Biol 18, 5308-5319.

Scherz-Shouval, R.und Elazar, Z. (2007). ROS, mitochondria and the regulation of autophagy. Trends Cell Biol 17, 422-427.

Schuberth, C.und Buchberger, A. (2008). UBX domain proteins: major regulators of the AAA ATPase Cdc48/p97. Cell. Mol. Life Sci. 65, 2360-2371.

Schuberth, C., Richly, H., Rumpf, S.und Buchberger, A. (2004). Shp1 and Ubx2 are adaptors of Cdc48 involved in ubiquitin-dependent protein degradation. EMBO Rep 5, 818-824.

Scott, S.V., Baba, M., Ohsumi, Y.und Klionsky, D.J. (1997). Aminopeptidase I is targeted to the vacuole by a nonclassical vesicular mechanism. The Journal of Cell Biology 138, 37-44.

Scott, S.V., Nice, D.C., 3rd, Nau, J.J., Weisman, L.S., Kamada, Y., Keizer-Gunnink, I., Funakoshi, T., Veenhuis, M., Ohsumi, Y.und Klionsky, D.J. (2000). Apg13p and Vac8p are part of a complex of phosphoproteins that are required for cytoplasm to vacuole targeting. $\mathrm{J}$ Biol Chem 275, 25840-25849.

Sesaki, H.und Jensen, R.E. (2004). Ugolp links the Fzolp and Mgm1p GTPases for mitochondrial fusion. J Biol Chem 279, 28298-28303.

Shaw, J.M.und Nunnari, J. (2002). Mitochondrial dynamics and division in budding yeast. Trends Cell Biol 12, 178-184.

Sheng, Y., Mancino, V.und Birren, B. (1995). Transformation of Escherichia coli with large DNA molecules by electroporation. Nucleic Acids Res 23, 1990-1996.

Shibata, M., Lu, T., Furuya, T., Degterev, A., Mizushima, N., Yoshimori, T., MacDonald, M., Yankner, B.und Yuan, J. (2006). Regulation of intracellular accumulation of mutant Huntingtin by Beclin 1. J Biol Chem 281, 14474-14485.

Shintani, T., Huang, W.P., Stromhaug, P.E.und Klionsky, D.J. (2002). Mechanism of cargo selection in the cytoplasm to vacuole targeting pathway. Dev Cell 3, 825-837.

Sikorski, R.S.und Hieter, P. (1989). A system of shuttle vectors and yeast host strains designed for efficient manipulation of DNA in Saccharomyces cerevisiae. Genetics 122, 1927.

Smith, D.B.und Johnson, K.S. (1988). Single-step purification of polypeptides expressed in Escherichia coli as fusions with glutathione S-transferase. Gene 67, 31-40.

Strittmatter, C.F. (1957). Adaptive variation in the level of oxidative activity in Saccharomyces cerevisiae. J Gen Microbiol 16, 169-183.

Strømhaug, P.E., Reggiori, F., Guan, J., Wang, C.W.und Klionsky, D.J. (2004). Atg21 is a phosphoinositide binding protein required for efficient lipidation and localization of Atg8 during uptake of aminopeptidase I by selective autophagy. Mol Biol Cell 15, 3553-3566. 
Suzuki, K., Kirisako, T., Kamada, Y., Mizushima, N., Noda, T.und Ohsumi, Y. (2001). The pre-autophagosomal structure organized by concerted functions of APG genes is essential for autophagosome formation. EMBO J 20, 5971-5981.

Suzuki, K., Kondo, C., Morimoto, M.und Ohsumi, Y. Selective transport of alphamannosidase by autophagic pathways: identification of a novel receptor, Atg34p. J Biol Chem 285, 30019-30025.

Suzuki, K., Kubota, Y., Sekito, T.und Ohsumi, Y. (2007). Hierarchy of Atg proteins in preautophagosomal structure organization. Genes Cells 12, 209-218.

Swaminathan, S., Amerik, A.Y.und Hochstrasser, M. (1999). The Doa4 deubiquitinating enzyme is required for ubiquitin homeostasis in yeast. Mol Biol Cell 10, 2583-2594.

Sychrova, H.und Chevallier, M.R. (1993). Cloning and sequencing of the Saccharomyces cerevisiae gene LYP1 coding for a lysine-specific permease. Yeast 9, 771-782.

Takeshige, K., Baba, M., Tsuboi, S., Noda, T.und Ohsumi, Y. (1992). Autophagy in yeast demonstrated with proteinase-deficient mutants and conditions for its induction. The Journal of Cell Biology 119, 301-311.

Tal, R., Winter, G., Ecker, N., Klionsky, D.J.und Abeliovich, H. (2007). Auplp, a yeast mitochondrial protein phosphatase homolog, is required for efficient stationary phase mitophagy and cell survival. J Biol Chem 282, 5617-5624.

Tatsuta, T.und Langer, T. (2008). Quality control of mitochondria: protection against neurodegeneration and ageing. EMBO J 27, 306-314.

Terzioglu, M.und Galter, D. (2008). Parkinson's disease: genetic versus toxin-induced rodent models. FEBS J 275, 1384-1391.

Tong, A.H.und Boone, C. (2006). Synthetic genetic array analysis in Saccharomyces cerevisiae. Methods Mol Biol 313, 171-192.

Tong, A.H., Evangelista, M., Parsons, A.B., Xu, H., Bader, G.D., Page, N., Robinson, M., Raghibizadeh, S., Hogue, C.W., Bussey, H., Andrews, B., Tyers, M.und Boone, C. (2001). Systematic genetic analysis with ordered arrays of yeast deletion mutants. Science 294, 23642368 .

Towbin, H., Staehelin, T.und Gordon, J. (1979). Electrophoretic transfer of proteins from polyacrylamide gels to nitrocellulose sheets: procedure and some applications. Proc Natl Acad Sci U S A 76, 4350-4354.

Trumbly, R.J. (1992). Glucose repression in the yeast Saccharomyces cerevisiae. Mol Microbiol 6, 15-21.

Tucker, K.A., Reggiori, F., Dunn, W.A., Jr.und Klionsky, D.J. (2003). Atg23 is essential for the cytoplasm to vacuole targeting pathway and efficient autophagy but not pexophagy. J Biol Chem 278, 48445-48452. 
Tuttle, D.L.und Dunn, W.A., Jr. (1995). Divergent modes of autophagy in the methylotrophic yeast Pichia pastoris. J Cell Sci 108 ( Pt 1), 25-35.

Twig, G., Hyde, B.und Shirihai, O.S. (2008). Mitochondrial fusion, fission and autophagy as a quality control axis: the bioenergetic view. Biochim Biophys Acta 1777, 1092-1097.

Veenhuis, M., Douma, A., Harder, W.und Osumi, M. (1983). Degradation and turnover of peroxisomes in the yeast Hansenula polymorpha induced by selective inactivation of peroxisomal enzymes. Arch Microbiol 134, 193-203.

Wallace, D.C. (2005). A mitochondrial paradigm of metabolic and degenerative diseases, aging, and cancer: a dawn for evolutionary medicine. Annu Rev Genet 39, 359-407.

Wang, C.W., Kim, J., Huang, W.P., Abeliovich, H., Stromhaug, P.E., Dunn, W.A., Jr.und Klionsky, D.J. (2001). Apg2 is a novel protein required for the cytoplasm to vacuole targeting, autophagy, and pexophagy pathways. J Biol Chem 276, 30442-30451.

Wang, C.W., Stromhaug, P.E., Kauffman, E.J., Weisman, L.S.und Klionsky, D.J. (2003). Yeast homotypic vacuole fusion requires the Cczl-Mon1 complex during the tethering/docking stage. J Cell Biol 163, 973-985.

Weber, T.A.und Reichert, A.S. (2010). Impaired quality control of mitochondria: aging from a new perspective. Exp Gerontol 45, 503-511.

Welter, E., Thumm, M.und Krick, R. (2010). Quantification of nonselective bulk autophagy in S. cerevisiae using Pgk1-GFP. Autophagy 6, 794-797.

Wong, E.D., Wagner, J.A., Gorsich, S.W., McCaffery, J.M., Shaw, J.M.und Nunnari, J. (2000). The dynamin-related GTPase, Mgm1p, is an intermembrane space protein required for maintenance of fusion competent mitochondria. J Cell Biol 151, 341-352.

$\mathrm{Wu}$, K.K. (2006). Analysis of protein-DNA binding by streptavidin-agarose pulldown. Methods Mol Biol 338, 281-290.

Wurmser, A.E.und Emr, S.D. (2002). Novel PtdIns(3)P-binding protein Etf1 functions as an effector of the Vps34 PtdIns 3-kinase in autophagy. The Journal of Cell Biology 158, 761772 .

Xie, Z., Nair, U.und Klionsky, D.J. (2008a). Atg8 controls phagophore expansion during autophagosome formation. Mol Biol Cell 19, 3290-3298.

Xie, Z., Nair, U.und Klionsky, D.J. (2008b). Dissecting autophagosome formation: the missing pieces. Autophagy 4, 920-922.

Xilouri, M., Vogiatzi, T., Vekrellis, K.und Stefanis, L. (2008). alpha-synuclein degradation by autophagic pathways: a potential key to Parkinson's disease pathogenesis. Autophagy 4, 917-919.

Yang, Q., She, H., Gearing, M., Colla, E., Lee, M., Shacka, J.J.und Mao, Z. (2009). Regulation of neuronal survival factor MEF2D by chaperone-mediated autophagy. Science $323,124-127$. 
Ye, Y., Meyer, H.H.und Rapoport, T.A. (2001). The AAA ATPase Cdc48/p97 and its partners transport proteins from the ER into the cytosol. Nature 414, 652-656.

Yen, W.L., Legakis, J.E., Nair, U.und Klionsky, D.J. (2007). Atg27 is required for autophagy-dependent cycling of Atg9. Mol Biol Cell 18, 581-593.

Yorimitsu, T.und Klionsky, D.J. (2005). Autophagy: molecular machinery for self-eating. Cell Death Differ 12 Suppl 2, 1542-1552.

Zanchin, N.I.und Goldfarb, D.S. (1999). Nip7p interacts with Nop8p, an essential nucleolar protein required for $60 \mathrm{~S}$ ribosome biogenesis, and the exosome subunit Rrp43p. Mol Cell Biol 19, 1518-1525.

Ziviani, E., Tao, R.N.und Whitworth, A.J. (2010). Drosophila parkin requires PINK1 for mitochondrial translocation and ubiquitinates mitofusin. Proc Natl Acad Sci U S A 107, 5018-5023. 


\section{DANKSAGUNG}

Hiermit möchte ich ganz besonders Herrn Prof. Thumm für die Bereitstellung dieses sehr interessanten Themas und für die gute Betreuung danken.

Bei Herrn Prof. Braus möchte ich mich für die Tätigkeit als Co-Referent bedanken.

Weiterhin möchte ich mich allen Mitgliedern meines Thesis Komitees: Herrn Prof. Thumm, Herrn Prof. Braus und Herrn Prof. Ficner für alle Ratschläge und Anregugnen bedanken.

Vielen Dank an Herrn Prof. Rehling für wissenschaftliche Ratschläge, besonders auf dem Gebiet der Mitophagy.

Mein besonderer Dank gilt Rosi, die mir im Laufe der Jahre immer wieder mit Rat und Tat zur Seite stand. Ganz besonders in den letzten Monaten des Schreibens war sie mir mit ihrer Geduld und wissenschaftlicher Expertise eine sehr große Hilfe.

Der gesamten Arbeitsgruppe Thumm: Petra, Lisa, Rosi und Peter möchte ich danken, für das angenehme Arbeitsklima, die wissenschaftlichen Diskussionen und vor allem für das freundschaftliche Miteinander während und außerhalb der Arbeitszeiten. Auch meine ehemaligen Kollegen Sebastian und Jörn sind an dieser Stelle natürlich nicht vergessen. Vielen Dank auch für das Korrekturlesen der Arbeit.

Allen anderen Mitgliedern der Biochemie II und I möchte ich ebenfalls für viele wissenschaftlich-hilfreiche Tipps und die vielen netten Gespräche privater Natur danken.

Ein großer Dank geht an meine Freunde. Vor allem Conny, Anja, Antje und Janine möchte ich hier besonders hervorheben. Vielen Dank für die vielen Telefonate und das offene Ohr, das ihr immer für mich hattet.

Ganz herzlich möchte ich meiner Mutter danken, für die finanzielle und vor allem mentale und emotionale Unterstützung während meines Studium und meiner Arbeit hier in Göttingen. Ein großer Dank geht an meine Oma, meine Omi, meine Schwester, meinen Neffen und mein Nichte. Vielen Dank auch an meinen Opa, der mir immer ein großes Vorbild war und sicherlich sehr stolz auf mich wäre.

DANKE! 


\section{CURRICULUM VITAE}

Personal Data

\begin{tabular}{ll}
\hline Name & Evelyn Cornelia Welter \\
Born & 15.04 .1982 in Ottweiler/Germany
\end{tabular}

Education

10.2007-present

Dissertation at the Georg-August-Universtiy of Göttingen

Department of Biochemistry II, Center for Biochemistry and Molecular Cell Biology, Prof. Dr. Michael Thumm

$11.2001-07.2007$

Studies in Biology at the University of Rostock

Major subject: Biochemistry

Minor subjects: Molecular biology and Microbiology

Diploma thesis at the University of Rostock

Charakterisierung der Linaloolsynthase aus Nicotiana alata

Department: Biochemie der Pflanze, Prof. Dr. Birgit Piechulla

1994- 2001

Gymnasium Ottweiler

1988- $1994 \quad$ Grundschule Lebesch, Ottweiler

\section{Publications}

Welter, E., Thumm, M. und Krick R. (2010). Quantification of nonselective bulk autophagy in S. cerevisiae using Pgk1-GFP. Autophagy 6, 794-797.

Krick, R., Bremer, S., Welter, E., Schlotterhose, P., Muehe, Y., Eskelinen, E.L.und Thumm, M. (2010). Cdc48/p97 and Shp1/p47 regulate autophagosome biogenesis in concert with ubiquitin-like Atg8. J Cell Biol 190, 965-973.

Krick, R., Bremer, S., Welter, E., Eskelinen, E.L.und Thumm, M. (2011). Cheating on ubiquitin with Atg8. Autophagy 7. 